\title{
ANALYTICAL METHOD DEVELOPMENT TO PREDICT THE IN-RUBBER DISPERSIBILITY OF SILICA
}

Fabian Grunert 


\section{Graduation committee:}

Chairman:

Secretary:

Promotor:

Internal members:

External member:

Referee:

Special expert:
Prof. Dr. G.P.M.R. Dewulf Prof. Dr. G.P.M.R. Dewulf Prof. Dr. A. Blume

Dr. W.K. Dierkes

Prof. Dr. Ir. J. E. ten Elshof

Prof. Dr. Dariusz Bieliński

Prof. Dr. Volker Herrmann

Dr. Joachim Bertrand

Mr. André Wehmeier
University of Twente, ET

University of Twente, ET

University of Twente, ET/ETE

University of Twente, ET/ETE

University of Twente, TNW/IMS

Lodz University of Technology, Poland

University of Applied Science Würzburg-

Schweinfurt, Germany (FHWS)

Behn Meyer Europa GmbH, Germany

Evonik Resource Efficiency GmbH, Germany 


\title{
ANALYTICAL METHOD DEVELOPMENT TO PREDICT THE IN-RUBBER DISPERSIBILITY OF SILICA
}

\author{
DISSERTATION
}

to obtain

the degree of doctor at the University of Twente, on the authority of the rector magnificus, prof.dr. T.T.M. Palstra,

on account of the decision of the Doctorate Board,

to be publicly defended

on Wednesday, the $7^{\text {th }}$ of December, 2018 at 16:45 hours

By

\section{Fabian Grunert}

born on the $17^{\text {th }}$ of January, 1988

in Würzburg, Germany 
This dissertation has been approved by:

Promotor: Prof. Dr. A. Blume

Printed by: Ipskamp Printing B.V., Postbus 333, 7500 AH Enschede, the Netherlands ISBN: 978-90-365-4655-3

DOI: $10.3990 / 1.9789036546553$

(C) 2018 Fabian Grunert, The Netherlands. All rights reserved. No parts of this thesis may be reproduced, stored in a retrieval system or transmitted in any form or by any means without permission of the author.

Alle rechten voorbehouden. Niets uit deze uitgave mag worden vermenigvuldigd, in enige vorm of op enige wijze, zonder voorafgaande schriftelijke toestemming van de auteur. 
To the best mum in the world... mine! 



\section{Table of contents}

$\begin{array}{lll}\text { Chapter 1: } & \text { Introduction }\end{array}$

$\begin{array}{ll}\text { Chapter 2: } \quad \text { Literature Review } & 5\end{array}$

$\begin{array}{lll}\text { Chapter 3: } & \text { Rubber Compounding and Testing }\end{array}$

Chapter 4.1: $\quad$ The Principles of Method Development 67 and Sample Preparation

Chapter 4.2: $\quad$ Method Development 1:

Sedimentation Analysis

Chapter 4.4: $\quad$ Method Development 3:

In-situ Cluster Fragmentation

Chapter 6: $\quad$ Discussion and Conclusion -

Investigation of the Morphology of Silica

Chapter 7: $\quad$ Summary and Samenvatting

Bibliography 



\section{Chapter 1 - Introduction}

The present chapter provides a short background for the investigations conducted in this work. The motivation and aim for the research is given as well as an overview to the structure of the thesis.

\subsection{Background of the Investigations}

Since the introduction of the "Green-Tire" by Michelin in 1992 [1], precipitated silica in combination with bi-functional organosilanes became one of the most important fillers for passenger car tire tread compounds. This filler system leads, in combination with a special polymer system (high $T_{g}$ solution-styrene-butadiene copolymer and a low $T_{g}$ 1,4-polybutadiene), to a better wet traction and lower rolling resistance in comparison to carbon black filled treads (Fig. 1.1). This results in a higher safety performance and a lower fuel consumption. However, up to now it is still challenging to obtain an equivalent or even improved level of abrasion resistance which would improve the service life of a tire and, in the end, reduces the amount of scrap tires per year [2].

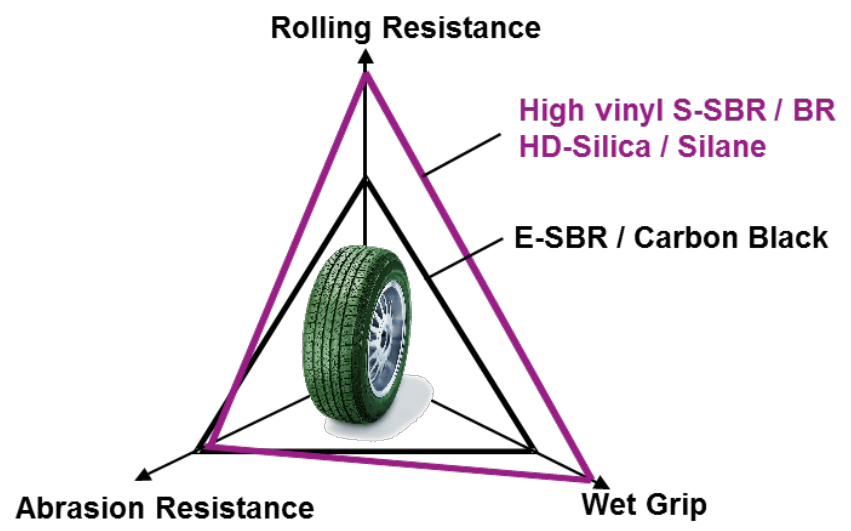

Figure 1.1: The Magic Triangle to depict the tire performance rating of carbon black and Highly Dispersible (HD) silica filled tread compounds [2]

Investigations of real tire tests [3] show that the abrasion resistance of silica filled passenger car tire treads is strongly influenced by the macro-dispersion quality of the filler, which was already claimed by Medalia [4]. It turned out that a better macro-dispersion, which means less undispersed filler-particles, results in a higher abrasion resistance (Fig. 1.2).
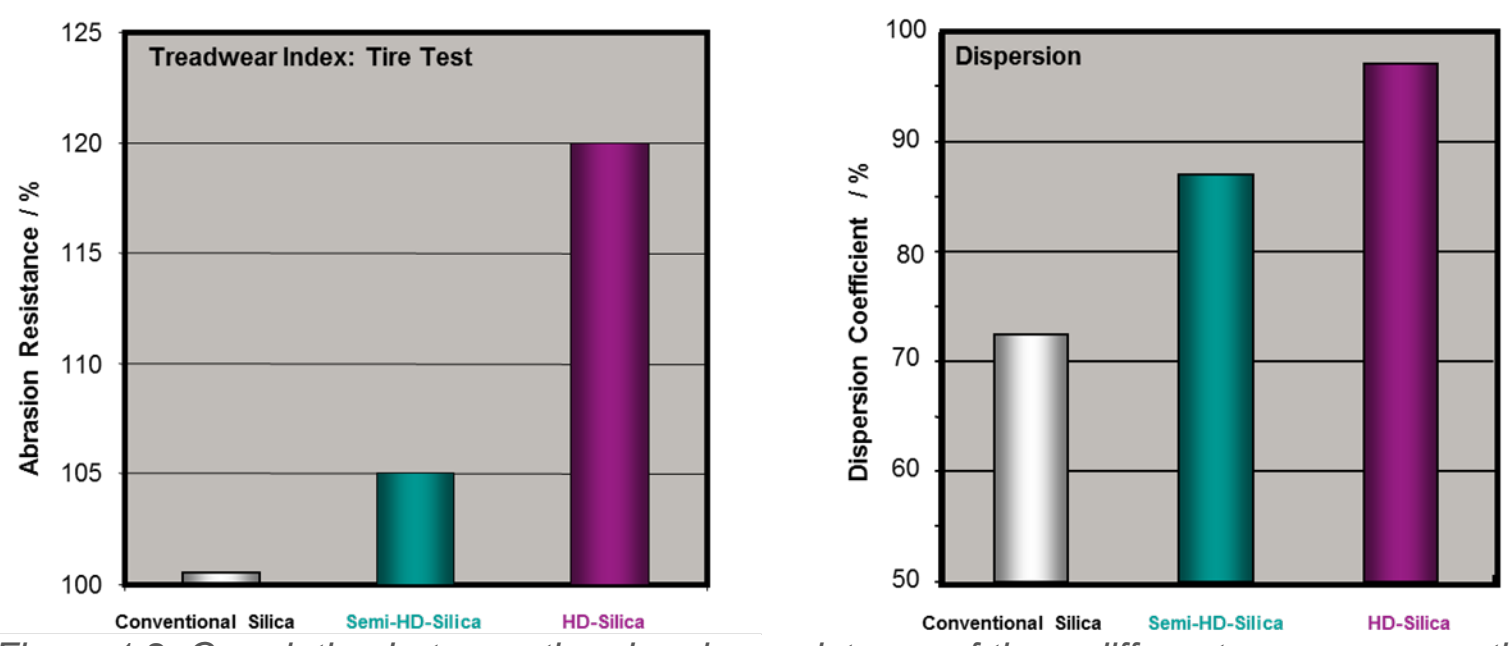

Figure 1.2: Correlation between the abrasion resistance of three different passenger car tire treads and the macro-dispersion quality of silica [3] 
The expression "dispersion" is defined as the "degree of uniform distribution of a filler's primary unit (i.e., aggregate of carbon black) into a compound" and "macro-dispersion" characterizes the dispersion quality in the size range between 2 and $100 \mu \mathrm{m}$ [5]. To improve the macrodispersion and therefore the abrasion resistance of passenger car tire tread compounds it is crucial to understand how the dispersion quality of silica inside a rubber compound can be improved.

\subsection{Aim of the Research}

Previous works [6-7] showed that the compound formulation and the mixing process have a great impact on the in-rubber dispersion quality of silica. Some of those influencing parameters are contradictory. The silane for instance hydrophobizes the silica surface. This process is named the "silanization reaction". As a result, the filler-filler interactions are reduced and the dispersion behavior is improved. Therefore, a fast and sufficient silanization reaction is desired. A high mixing temperature and a longer mixing time can fulfill this requirement. In contrast, both mentioned mixing parameters increase the risk of a pre-scorch during the mixing [7]. Consequently, the chosen parameters for the mixing process represent always a compromise to achieve the objectives. Fig. 1.3 depicts the influences of the temperature $(T)$, the time $(\mathrm{t})$ and the compound viscosity $(\mathrm{n})$ on different processes during the mixing.

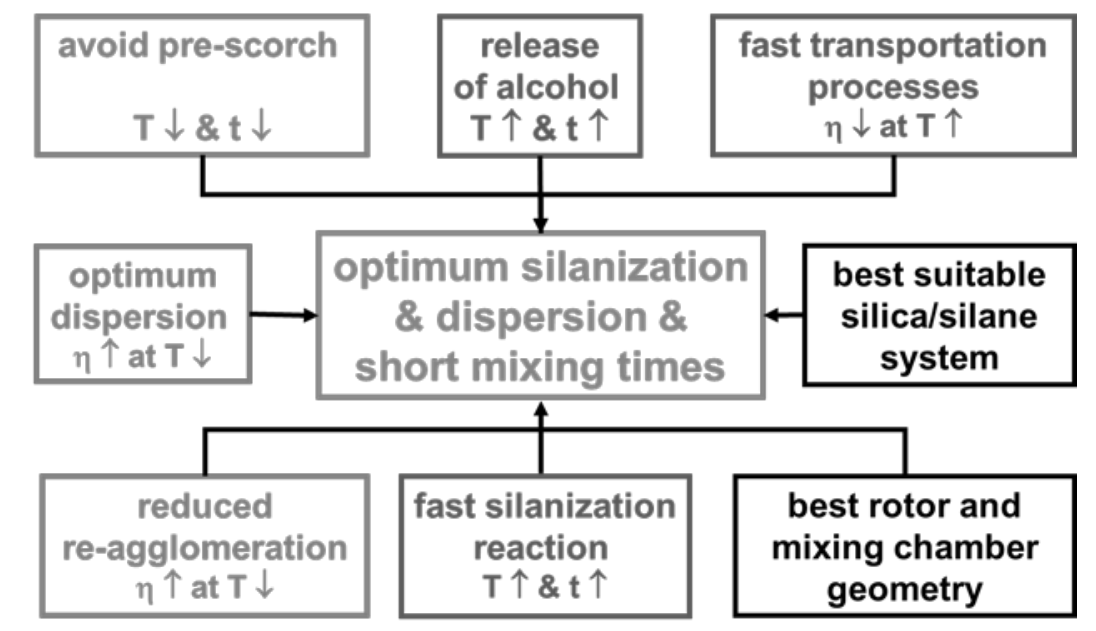

Figure 1.3: Conflicts of objectives during the mixing process [7]

The silica itself has a great impact on the in-rubber dispersion quality as well. In contrast to the above mentioned parameters, silica can be optimized regarding its dispersibility without facing any conflicts. The term "dispersibility" describes the ability of silica to be dispersed into a rubber matrix [8]. It can only be assessed within an identical compound formulation and mixing process and is exclusively be influenced by the filler properties. Considering, that these parameters are known, it would be possible to adjust them precisely. Thus tailor-made silica could be designed which show an improved dispersibility.

To develop new highly dispersible silica it is crucial to be aware of the typical analytical silica parameters and their impact on the dispersion process (Tab. 1.1). A higher surface area for instance enhances the filler-filler interactions via hydrogen bonds and results in a pronounced flocculation of silica clusters forming a strong filler-filler network. To break these clusters down higher shear forces are required. As a consequence, the incorporation and distributive processes are more difficult and lead to a worse dispersion quality [7]. 
Table 1.1: Typical analytical parameters and influences on dispersion process [9]

\begin{tabular}{|c|c|c|c|}
\hline Method & Norm & Description & Influence on \\
\hline BET*: specific surface area & ISO 9277 & $\begin{array}{c}\text { adsorption of nitrogen: } \\
\text { internal and external } \\
\text { surface area }\end{array}$ & \multirow[t]{2}{*}{$\begin{array}{l}\text { filler-filler interac- } \\
\text { tions / dispersion }\end{array}$} \\
\hline CTAB $^{\star \star}$ : specific surface area & $\begin{array}{c}\text { ISO } \\
5794 / 1 G\end{array}$ & $\begin{array}{l}\text { adsorption of CTAB: } \\
\text { external surface area }\end{array}$ & \\
\hline DOA: initial structure & ISO 19246 & $\begin{array}{l}\text { void volume: ability to } \\
\text { absorb dioctyladipate }\end{array}$ & $\begin{array}{c}\text { incorporation / } \\
\text { dispersion }\end{array}$ \\
\hline moisture content $\left(2 \mathrm{~h} / 105^{\circ} \mathrm{C}\right)$ & ISO $787 / 2$ & $\begin{array}{l}\text { content of volatile com- } \\
\text { ponents }\end{array}$ & $\begin{array}{l}\text { silica/silane reac- } \\
\text { tion }\end{array}$ \\
\hline $\mathrm{pH}$ & ISO $787 / 9$ & $\begin{array}{c}\mathrm{H}^{+} / \mathrm{OH}^{-} \text {in aqueous so- } \\
\text { lution }\end{array}$ & $\begin{array}{l}\text { silica/silane reac- } \\
\text { tion }\end{array}$ \\
\hline sieve residue (Mocker) & ISO $787 / 18$ & $\begin{array}{l}\text { coarse particles re- } \\
\text { maining on the sieve af- } \\
\text { ter water treatment }\end{array}$ & $\begin{array}{l}\text { dispersion / rub- } \\
\text { ber surface de- } \\
\text { fects }\end{array}$ \\
\hline sieve analysis (Rotap) & $\begin{array}{c}\text { ISO } \\
5794 / 1 \mathrm{~F}\end{array}$ & $\begin{array}{l}\text { particle size distribution } \\
(\mu \mathrm{m}-\mathrm{mm}) \text { of granules }\end{array}$ & $\begin{array}{l}\text { granules stability } \\
\text { for conveying / in- } \\
\text { corporation }\end{array}$ \\
\hline
\end{tabular}

${ }^{*}$ Brunauer, Émmett and Teller

** cetyltrimethylammonium bromide

Up to now, no direct correlation between one single analytical silica parameter and its dispersibility is known. For that reason, it is desirable to develop a new analytical method to predict the in-rubber dispersibility of silica. This parameter can only be defined when different types of silica are used in an identical compound formulation using an identical mixing process. The analytical method should have a proper repeatability and reproducibility and should be able to distinguish between different types of silica.

\subsection{Structure of the Thesis}

The research of this thesis deals with the development of three different analytical methods to characterize precipitated silica for tire tread applications. A variety of silica with different analytical parameters are investigated and their effect on in-rubber properties within different compounds was evaluated. Finally, a correlation analysis between analytical parameters of silica and their effect on in-rubber properties was carried out. The overall goal is to predict the in-rubber dispersibility of silica by means of one single analytical parameter. The thesis consists of eight different chapters which are briefly summarized as follows:

Chapter 2 provides a general overview about rubber technology with an emphasis on silica dispersion, the dispersion quality and its impact on the final in-rubber properties. A basic understanding of the silica chemistry and morphology is given as well as its influences on inrubber properties.

Chapter 3 summarizes the standard analytical parameters of all types of silica used during the investigations. In addition, the list of formulations and ingredients of all rubber compounds including the mixing procedure is provided. The mixing curves and in-rubber tests are evaluated and conspicuous differences within the results are highlighted.

Chapter 4.1 provides an inside into the principles of analytical method development. A special emphasize is put on the sample preparation, storage effects, influence of different dosage forms and moreover on the use of ultrasound to reduce the cluster size of silica.

Chapter 4.2 focuses on the investigation of silica by means of the void volume structure tester. A suitable measurement procedure and evaluation method to determine the initial structure of silica is developed. 
Chapter 4.3 introduces a new method to measure the particle size and distribution of silica. The sedimentation method thereby records the amount of coarse particles inside a silica/water solution via $x$-ray absorption after a defined energy input.

Chapter 4.4 presents a third approach to predict the in-rubber dispersibility of silica. The in-situ cluster fragmentation method measures the reduction of particle sizes during an ultrasonic treatment. In this way the easiness and speed of silica-cluster breakage can be assessed.

Chapter 5 provides the linear correlations of the standard analytical parameters of silica as well as the received parameters by means of the three new developed methods with the inrubber test results. The analytical parameters which affects the dispersion quality the most, are evaluated. Finally, an equation including several parameters is proposed to predict in-rubber dispersibility of silica.

Chapter 6 discusses the outcome of the newly developed analytical methods respectively their evaluation parameters with the support of additional investigations to gain new insights into the structure of silica. A new approach to describe the morphology of silica is made to gain a better understanding of the dispersibility of silica.

\subsection{References}

[1] R. Rauline, Compagnie Generale des Establissements Michelin et Cie, Rubber compound and tires based on such a compound, EP 0501227 B1 (1991)

[2] M. F. Sheridan, Rubber Handbook, 14, R.T. Vanderbilt Company, Inc., Norwalk, CT (2010)

[3] S. Uhrlandt; A. Blume, Kieselsäure für den Grünen Reifen - Prozesse, Produkte, Eigenschaften, Kautsch. Gummi Kunstst. 54 (2001) 520-527

[4] A. I. Medalia, Microscopic estimation of carbon black dispersion, Rubber Age (1965) 82

[5] ASTM D3053:2015 - Standard Terminology Relating to Carbon Black

[6] H.-D. Luginsland, A Review on the Chemistry and the Reinforcement of the Silica-Silane Filler System for Rubber Applications, Shaker Verlag, Cologne (2002)

[7] B. Rodgers, Rubber Compounding - Chemistry and Applications, CRC Press, Boca Raton - London - New York (2016)

[8] F. Grunert; A. Wehmeier; A. Blume, Prediction of In-Rubber Dispersibility of Silica by Analytical Methods, presented at: 12th Fall - Rubber - Colloquium, Hannover (2016)

[9] A. Wehmeier, Determination of the Macro-Dispersion of Advanced Fillers in Rubber Compounds, Evonik Industries Customer Seminar, Wesseling, Germany (2015) 


\section{Chapter 2 - Literature Review}

This chapter introduces a fundamental overview over rubber technology and relevant topics investigated in this thesis. A basic understanding of the silica chemistry and morphology is given as well as their influences on in-rubber properties. The main focus is on silica dispersion and the dispersion quality.

\subsection{Rubber Technology}

\section{History of Rubber}

The first documented evidence of rubber being used is dated back to the sixth century where Central American natives used natural rubber for instance as shoes by immersing their feet into the latex to get a perfect fitted coverage. However, it was Christopher Columbus who described this raw material in detail for the first time back in the fifteenth century. Years later in 1736 Charles de la Condamine sent samples of the "Cahuchu" from an expedition in Peru to the French Academy of Science and thereby introduced this material to the western world [1]. The name "rubber" was invented by Edward Nairne, an English optician and scientific instrument maker who accidentally picked up a piece of rubber instead of usually used breadcrumbs to erase or "rub off" marks from lead pencils and was published by Joseph Priestley in 1770 [2]. The first real technical application was invented by Charles Macintosh back in 1823 who combined raw rubber with fabrics to achieve a water proof material for clothes. Eventually, it was Charles Goodyear who realized the major breakthrough in rubber technology by accidently discovering the vulcanisation process in 1839. He mixed a piece of natural rubber with sulphur amongst other ingredients and left it next to a hot oven. Parts of this mixture which were in direct contact to the heat turned into a softer, non-sticky and elastic material which he described later on in a patent in 1844 [1,3]. From that moment on the industrial application of rubber products started to rise. In 1888 John Boyd Dunlop developed the first air inflated tire for bicycles [4] before the brothers André and Édouard Michelin took over the idea for a passenger car tire in 1894 [1]. The next major step was the discovery of the reinforcing effect of carbon black in rubber (1902) and the plant scale production of synthetic rubber by Bayer in 1911 [5]. With natural rubber not being the only source for elastomers anymore, the use of reinforcing fillers and the knowledge of the vulcanisation process it was possible to create rubber products for much broader fields of application. However, the last big step forward was the introduction of the "Green-Tire" technology by Michelin in 1992 [6], where precipitated silica in combination with bi-functional organosilanes became one of the most important filler system for passenger car tire tread compounds. This filler system leads, in combination with a special polymer system, to a better wet traction and lower rolling resistance in comparison to carbon black filled treads [7].

\section{Rubber in general}

Polymers consisting of a number of repetitive monomer units forming macromolecules and having a glass transition temperature $T g<0{ }^{\circ} \mathrm{C}$ are referred to as rubber. At room temperature they almost behave like a very highly viscous liquid. These polymers can be chemically crosslinked (vulcanized) forming a wide-meshed three-dimensional network which turns them into "elastomers" or simply speaking "rubber" which is defined in ASTM D1566 [8]. They are insoluble in solvents, usually amorphous, incompressible, show a high elasticity, have a relatively low modulus in comparison to other technical materials and do not have any flow area at higher temperatures before they decompose. The polymer chains form flexible, statistically arranged coils at room temperature. Rubbers are known to be viscoelastic, which means that they show on the one hand viscous characteristics like a damping pot (Newtonian fluid) and on the other hand elastic characteristics like a spring (Hookean element) during a deformation. Below the glass transition temperature elastomers lose their rubber-like behavior and become hard and brittle, almost glassy. In accordance with DIN/ISO 1629 [9] rubbers are classified either due to the chemical construction of their backbone or their physical properties [1]. 


\section{Composition of a rubber compound}

Pure rubber itself is usually very limited in commercial applications due to its comparatively poor mechanical strenght. To turn an unvulcanized rubber into a highly technical product several requirements have to be met which are fulfilled by adding different ingredients. This process of choosing different materials and mixing them together is commonly known as "compounding". The fundamental properties of the final rubber compound like chemical and temperature resistance are given by the rubber itself, either by a single polymer or a polymer blend. The cross-linking system consists of the actual crosslinker and an accelerator system which determine the vulcanization time, speed and crosslink density. Additionally, mechanical, dynamical properties and stiffness as well as the aging behavior will be affected. Usually lubricants, like different oils, resins or liquid polymers are added to the compound to enhance the processing and optimize in-rubber properties as well. In addition, several ingredients like antioxidants, processing aids, pigments, resins and more can be used. However, next to the polymer itself, fillers or filler systems play a dominant role to determine the final product properties. They can mainly serve following purposes [1, 10-11]:

- reinforce the compound e.g. to increase strength

- dilute and extend the rubber to lower the costs

- color the rubber

- change the conductivity

To blend all components together they have to be mixed, commonly inside an internal mixer and/or on an open mill. The mixing process can be divided into several stages and the addition of the ingredients can take place at different times. The mixing process has to be optimized with respect to mixing time, temperature and energy consumption and has to fulfill certain purposes [10-11]:

- create a homogeneous compound

- disperse and distribute all ingredients added to the rubber

- in case of silica/silane-system: act as a reaction vessel

Due to the importancy of the mixing and the filler dispersion process for the present study these issues are discussed in detail in chapter 2.4. The final steps of the production process of elastomers are the moulding and the vulcanization. The most common process for vulcanization in the tire industry is the compression molding. The mixed compound is given into a preheated form and is pressed into shape. Therefore, it should have good flow properties to fill the mold completely without any trapped air left inside. By choosing appropriate chemicals during the compounding (e.g. sulfur, zinc oxide, fatty acid and accelerators) the crosslinking reaction takes place within minutes and the final rubber product is created [1, 10-11].

\subsection{Chemistry of Precipitated Silica}

The material "silica" is one of many possible appearances of the chemical composition silicon dioxide. $\mathrm{SiO}_{2}$ can be found most commonly in the form of quartz (crystalline structure) and roughly 13 weight- $\%$ of the earths outer layer consists of it. Silica can as well appear in nature with an amorphous structure, e.g. in form of opal gems. The first commercially available synthetic silica was invented by Harry Kloepfer (Degussa AG) in 1942 [10]. With the use of the high-temperature hydrolysis it was possible to produce pyrogenic silica, better known as AER$\mathrm{OSIL}^{\circledR}$. This type of silica has an open fractal structure, comparable to Carbon Black (CB) and appears as a very fluffy powder with a low bulk density. Therefore, it is difficult to mix with a solid rubber and is still mainly used to reinforce silicone rubber (liquid rubber). There are pyrogenic silica in a compacted form on the market to overcome this mixing issue but due to the higher price compared to precipitated silica, the use in other rubber types is very limited. The second synthetic produced type of silica was precipitated silica and was introduced by the Columbian Chemical Division of Pittsburgh Plate Glass Co. in 1948 [12]. Initially, precipitated silica was used for non-marking shoe soles and other applications where a transparent or colored appearance along with a high reinforcement was needed. 
About 20 years later, it was discovered that coupling agents, especially silanes, could be used in combination with silica to achieve appropriate reinforcing properties for high performance rubber compounds. Since the introduction of bis-(3-triethoxysilylpropyl)tetrasulfane (TESPT) to overcome scorch issues which appeared with other used silanes precipitated silica became of higher relevance for the rubber industry. The last big step forward was the introduction of the "Green-Tire" technology by Michelin in 1992 [6], where precipitated silica in combination with bi-functional organosilanes became one of the most important fillers for passenger car tire tread compounds. This filler system leads, in combination with a special polymer system, to a better wet traction and lower rolling resistance in comparison to carbon black filled tire treads $[1,7,11,13]$.

\section{Production Process of Precipitated Silica}

Two source materials are required to produce precipitated silica via a batch process. The first one is water glass respectively a water glass solution. To produce water glass, quartz sand is mixed with sodium carbonate and melted under high temperatures between $1200{ }^{\circ} \mathrm{C}$ up to $1500{ }^{\circ} \mathrm{C}$. The caused cullets $\left(\mathrm{Na}_{2} \mathrm{O} \cdot \mathrm{n} \mathrm{SiO}{ }_{2} ; \mathrm{n}=3-3.5\right)$ are solved in water and strong alkaline sodium silicate is obtained, better known as water glass. The second raw material is an acid, usually sulfuric acid. Fig. 2.1 shows a typical production line for precipitated silica, starting from water glass and sulfuric acid [11].

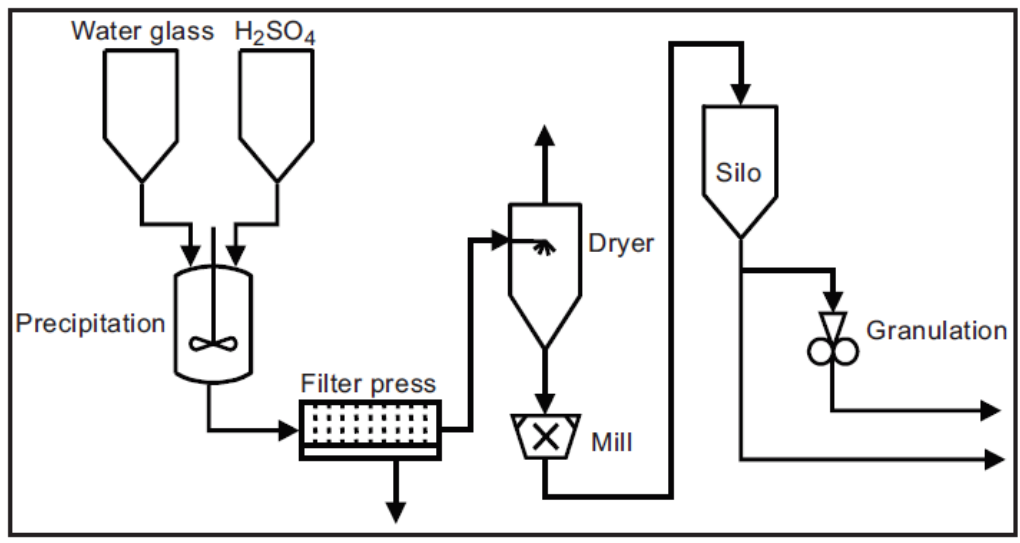

Figure 2.1: Production process of precipitated silica [11]

The production process can basically be divided into four steps: precipitation, filtration, drying and granulation (optional). Each step influences the final chemistry and morphology of silica and has to be controlled precisely to achieve the final product properties.

\section{Precipitation}

During the first step water glass and sulfuric acid are mixed inside the precipitation vessel resulting in a chemical reaction. Silica $\left(\mathrm{SiO}_{2}\right)$ precipitates and as a by-product water as well as sodium sulphate is obtained:

$$
\left(\mathrm{Na}_{2} \mathrm{O} \cdot \mathrm{n} \mathrm{SiO} 2\right)+\mathrm{H}_{2} \mathrm{SO}_{4} \Leftrightarrow \mathrm{n} \mathrm{SiO} 2+\mathrm{Na}_{2} \mathrm{SO}_{4}+\mathrm{H}_{2} \mathrm{O}
$$

This reaction is reversible and therefore has to be controlled very carefully. The formation of silica can be divided in two parts, the particle growth and the growth of aggregates and agglomerates which have to be balanced. Single silica primary particles increase in their diameter by means of condensation primarily at a low salt concentration whereas the formation of threedimensional structures prevails at higher salt concentrations [13].

\section{Filtration}

To separate the precipitated silica from the salt and other residuals the suspension which contains $5-10 \mathrm{wt} \%$ silica is transferred to a membrane filter press where it is compressed and washed/filtered with water. The obtained filter cake still contains up to $85 \mathrm{wt} \%$ of water and has therefore to be dried in a further step [14]. 


\section{Drying}

Silica is usually dried up to a free water content of $4-7 \mathrm{wt} \%$, which roughly corresponds to the equilibrium free water content at $50 \%$ relative humidity [14]. The most common types of dryers are rotary, spray, plate, spin-flash and spray nozzle tower dryers. Depending on the application silica can additionally be milled or compacted in form of e.g. granules in a final step after drying. With other techniques fine powder or small pearls are produced [14-15].

\section{Granulation}

The difficulties during the transportation of fluffy powdery silica as well as its difficult incorporation into rubber compounds causes inconveniences. Therefore, silica is compressed into granules (several $\mathrm{mm}$ in diameter) under a decent pressure which increases e.g. its bulk density. The following table (Tab. 2.1) gives an overview of these four production steps, their adjustable parameters and how they can influence the chemistry and morphology of the final silica product [11].

Table 2.1: Production steps of precipitated silica and their influences on the final product properties [11, 15]

\begin{tabular}{ccc}
\hline Production Step & Controllable Parameter & Influenced Properties \\
\hline Precipitation & $\begin{array}{c}\mathrm{pH} \text {-value, reaction temperature and } \\
\text { time, concentration, dosage and } \\
\text { mixing of educts }\end{array}$ & $\begin{array}{c}\text { Specific Surface Area (SSA), } \\
\text { structure, silanol group den- } \\
\text { sity, particle size and pore size } \\
\text { distribution }\end{array}$ \\
\hline Filtration & $\begin{array}{c}\text { filling, washing time, } \\
\text { solid content }\end{array}$ & $\begin{array}{c}\text { structure, pH-value, conductiv- } \\
\text { ity, pore size distribution }\end{array}$ \\
\hline Drying & $\begin{array}{c}\text { type of dryer, temperature, } \\
\text { solid content, time }\end{array}$ & $\begin{array}{c}\text { SSA, structure, moisture con- } \\
\text { tent, particle size and pore size } \\
\text { distribution }\end{array}$ \\
\hline Granulation & feed rate, pressure & $\begin{array}{c}\text { structure, sieve residue, bulk } \\
\text { density, particle size and pore } \\
\text { size distribution }\end{array}$ \\
\hline
\end{tabular}

\section{Morphology of Precipitated Silica}

The most common used model to describe the general morphology of silica is shown in Fig. 2.2. The smallest basic silica unit relevant for rubber reinforcement is an aggregate, a threedimensional fractal structure, which consists of primary particles covalently linked together via siloxane bonds. Single aggregates can usually be found in a size range of 50 up to $300 \mathrm{~nm}$ in diameter (d). Due to the high surface polarity of silica, aggregates form via hydrogen bonds loosely bonded agglomerates $(\mathrm{d}>200 \mathrm{~nm})$ [7].

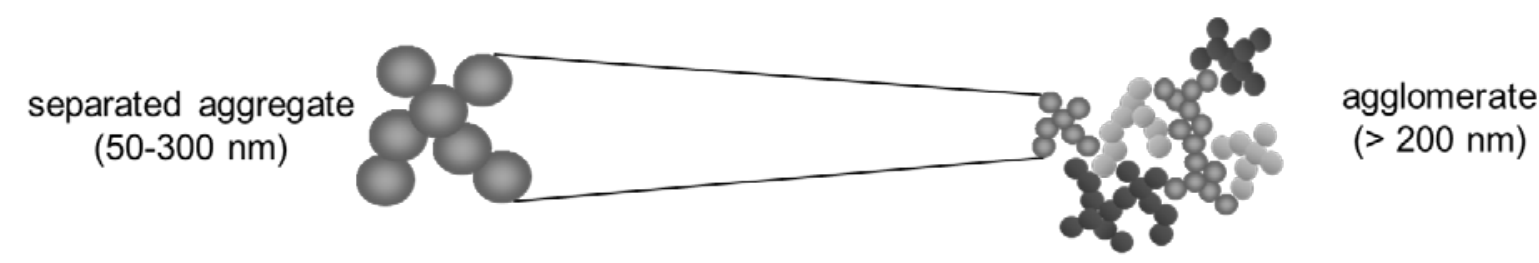

Figure 2.2: Typical model for the morphology of silica

To describe and distinguish between different types of precipitated silica three main characteristics of the silica are taken into consideration: the specific surface area (SSA), the initial structure and the surface chemistry.

\section{Specific Surface Area (SSA)}

The SSA of silica correlates with the size of the primary particles. The smaller the average diameter, the higher the actual surface area. To determine the SSA two different adsorption methods are used. The first is named BET in accordance with Brunauer, Emmett and Teller 
where nitrogen $\left(\mathrm{N}_{2}\right)$ is adsorbed on the surface of silica [16]. Due to the relatively small size of nitrogen molecules they are able to penetrate inside the porous structure of silica. Therefore, the calculated total surface area is a combination of the outer and inner surface (Fig. 2.3). A typical BET size range of silica for rubber applications is 50 to $250 \mathrm{~m}^{2} / \mathrm{g}$ [7].

The second method to determine the SSA is adopted from the Carbon Black analytics where CetylTrimethylAmmonium Bromide (CTAB) is adsorbed [17]. These molecules are larger than nitrogen and can only be adsorbed on the outer surface of silica. This outer surface area corresponds to the surface area accessible for the polymer chains to penetrate into and therefore is decisive for the reinforcement of rubber [18]. Typical CTAB values of silica for rubber applications are 50 to $175 \mathrm{~m}^{2} / \mathrm{g}$ [7]. Recently, newest developments tend to even higher surface areas up to $200 \mathrm{~m}^{2} / \mathrm{g}$ [19]. Fig. 2.3 illustrates the different accessibilities of the $\mathrm{N}_{2}$ and CTAB molecules on a porous surface of a silica cluster.

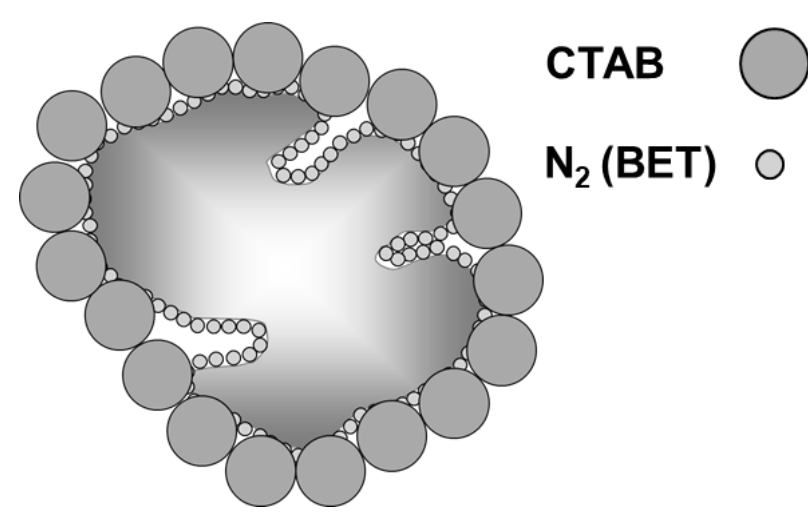

Figure 2.3: Description of the inner- and outer surface area of porous silica by $N_{2}(B E T)$ or CTAB measurement [15]

\section{Initial Structure}

The term "initial structure" characterizes the inter-aggregate three-dimensional assembly of silica particles. It is determined by the particle sizes and size distribution, total number of primary particles forming aggregates and the shape of the silica agglomerates. The initial structure is usually measured by an oil adsorption method whereby DiOctylAdipate (DOA) fills up the void volume (air in between the structure) of a filler [20]. This is based on the assumption that a higher void volume can be filled with a higher amount of oil which indicates a higher initial structure. Fig. 2.4 depicts two silica clusters with an identical SSA containing the same number of primary particles, one with a higher (I) and one with a lower (II) initial structure.
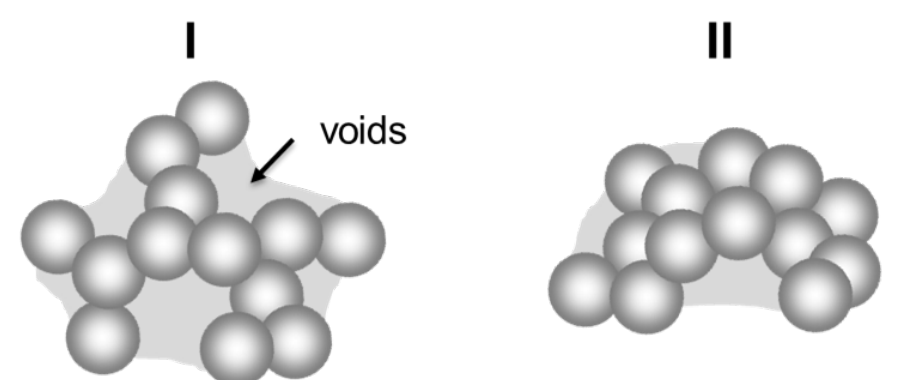

Figure 2.4: Silica with a higher (I) and a lower (II) initial structure

Beside the initial structure it is also possible to determine the "pore volume" and "pore size distribution" of silica. In principle, three different types of pores are distinguished:

- Micropores $<2 \mathrm{~nm}$ in diameter

- Mesopores $=2-50 \mathrm{~nm}$ in diameter

- Macropores >50 nm in diameter 
These pore sizes can be measured by means of "mercury porosimetry intrusion" whereby mercury is forced into the pores by increasing pressure [21]. The higher the pressure, the smaller pore diameters can be measured. And the higher the volume of mercury at a defined pressure, the higher the volume of these particular pores. Based on this, the pore size distribution can be calculated (pore volume as a function of pore diameter) [11].

\section{Surface chemistry}

The surface of precipitated silica is highly polar and consists of siloxane and silanol groups. In addition to the surface acidity and the amount of adsorbed water, the silanol groups mainly determine the chemistry of the silica surface [7]. These groups can be divided into three different types as shown in Fig. 2.5: geminal, isolated and vicinal silanol groups. Geminal ones appear the least but have a high reactivity. Isolated silanol groups have a high reactivity as well whereas the vicinal groups do not react with silane at all [22]. It is possible to distinguish these different types of silanol groups by means of ${ }^{29} \mathrm{Si}$ NMR (Nuclear Magnetic Resonance) and, with restriction, IR spectroscopy. Depending on the used measurement technique the silanol group density of precipitated silica turns out to be in between 4 to $10 \mathrm{SiOH} / \mathrm{nm}^{2}$ $[11,13]$.

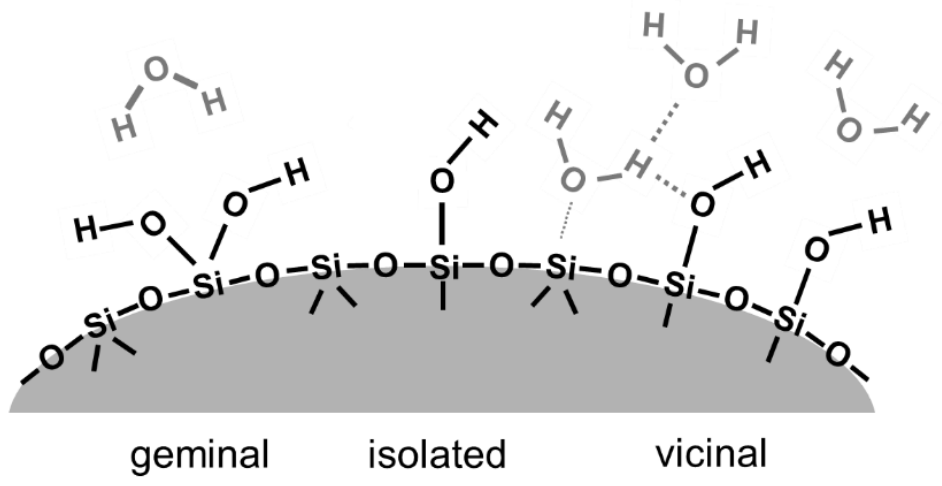

Figure 2.5: Different types of silanol groups on top of the surface of silica

A common approach to measure the amount of silanol groups on the silica surface is the determination of the Sears number. A silica suspension is set to a pH value of 6 and subsequently titrated with potassium hydroxide $(0.1 \mathrm{n} \mathrm{KOH})$ up to a pH value of 9 . The Sears number is given as the consumed amount of $\mathrm{KOH}$ in $\mathrm{ml} / 1.5 \mathrm{~g}$ [23].

In addition to mentioned measurement methods other techniques are used to investigate precipitated silica. Tab. 2.2 gives an overview over the most common methods and their purpose. 
Table 2.2: Common measurement methods to characterize silica

\begin{tabular}{|c|c|c|}
\hline Method & Norm & Description \\
\hline BET & ISO 9277 & inner and outer surface area \\
\hline CTAB & ISO $5794 / 1 G$ & outer surface area \\
\hline DBP / DOA number & $\begin{array}{l}\text { ASTM D } 1208 / \\
\text { ISO } 19246: 2016\end{array}$ & initial structure \\
\hline moisture content & ISO $787 / 2$ & content of volatile components \\
\hline pH - value & ISO $787 / 9$ & $\mathrm{H}^{+} / \mathrm{OH}^{-}$in aqueous solution \\
\hline Hg-Porosimetry & DIN 66133 & $\begin{array}{l}\text { pore size distribution } 500 \mu \mathrm{m}-5 \mu \mathrm{m} \\
\text { or } 15 \mu \mathrm{m}-3.5 \mathrm{~nm}\end{array}$ \\
\hline $\begin{array}{l}\text { CPS } \\
\text { disc centrifuge }\end{array}$ & ISO 20927 (E) & $\begin{array}{l}\text { particle size distribution } \\
\text { from } \mathrm{nm} \text { to } \mu \mathrm{m}\end{array}$ \\
\hline $\begin{array}{l}\text { sieve analysis } \\
\text { (Rotap) }\end{array}$ & ISO 5794/1F & $\begin{array}{l}\text { particle size distribution }(\mu m-m m) \text { of gran- } \\
\text { ules }\end{array}$ \\
\hline $\begin{array}{l}\text { sieve residue } \\
\text { (Mocker) }\end{array}$ & ISO $787 / 18$ & $\begin{array}{c}\text { Coarse particles remaining on the sieve after } \\
\text { water treatment }\end{array}$ \\
\hline SEARS - number & l & $\begin{array}{l}\text { number of silanol groups at the surface be- } \\
\text { ing detectable by a specific probe molecule }\end{array}$ \\
\hline conductivity & ISO $787 / 14$ & conductivity in aqueous solution \\
\hline
\end{tabular}

\subsection{Rubber Reinforcement}

\subsubsection{Filler classification}

The most common approach to classify fillers for the use in rubber applications is to distinguish between active and inactive fillers. Inactive fillers are often used to make a compound cheaper in a sense that the polymer matrix is diluted with the inexpensive filler without a major change in the compound properties. Usual primary particle sizes of inactive fillers are in the range of 500 to $1000 \mathrm{~nm}$. In contrast, the use of active fillers (usually 10 to $100 \mathrm{~nm}$ in particle size) can have a tremendous impact on the rubber characteristics. Therefore these active fillers are also known as reinforcing fillers [1].The activity in general depends on the rubber-filler interactions and their influences on the compound viscosity and mechanical properties e.g. tensile strength and elongation at break. These effects are related to the filler content. Inactive fillers, e.g. clays, show a linear change in properties with increasing amount of fillers whereas active fillers like silica and carbon black often show a maximum or minimum in the filler content as an optimum for a single compound property [1]. Fig. 2.6 depicts different compound properties in dependence of the type of filler and the filler content. 

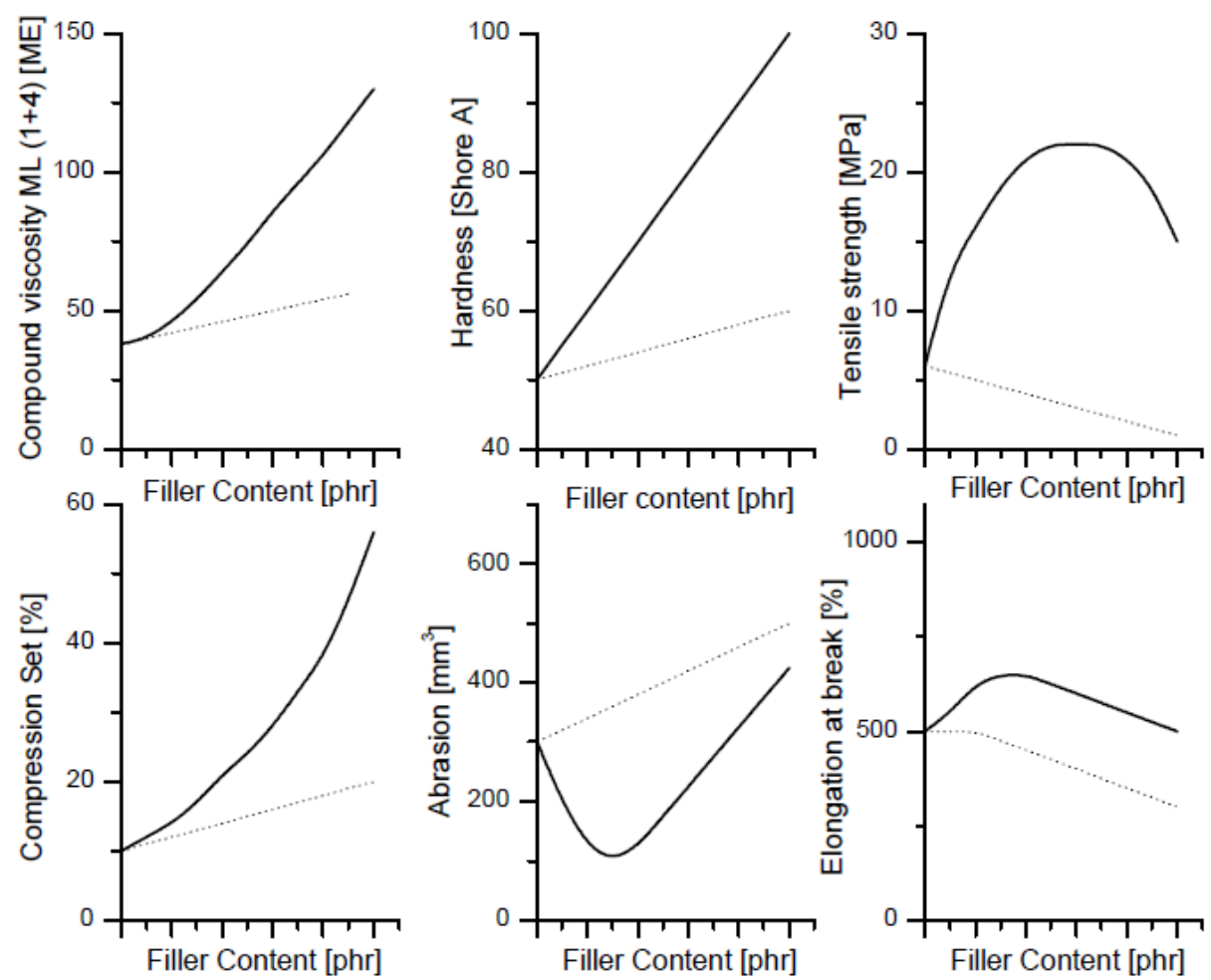

Figure 2.6: Different compound properties in dependency of type (.. inactive; - active) and amount of fillers [24]

\subsubsection{Rubber-Filler Reinforcement}

The term "reinforcement" was introduced by Wiegand in 1920 [25]. It is defined as the sum of all rubber-filler interactions influencing the physical properties of unvulcanized and vulcanized compounds. The area beneath the stress-strain curve (work) was described as a criterion to judge the level of reinforcement. Wiegand claimed that the reinforcing effect is determined by three factors: the particle size (surface factor), the particle shape (geometric factor) and the surface activity [26]. Nowadays, a variety of models and theories are used to describe the reinforcing mechanism of active fillers. They can in principle be subdivided into the structure and the adhesion theories.

\section{Structure models to describe the reinforcing effect Hydrodynamic effect}

The hydrodynamic effect in general describes the increase of a liquid's viscosity by the addition of solid/rigid spherical particles. It is assumed that the particles are considerably bigger than the polymer, no interactions between the filler particles occur, all particles are perfectly wetted by the liquid and the filler content is relatively low. This change in viscosity in dependence of the volume fraction can be calculated by equation 2.1 in accordance to Einstein [27-28]:

$$
\eta_{\Phi}=\eta \cdot(1+2.5 \cdot \Phi)
$$

Where $\eta_{\phi}$ represents the viscosity of the filled system, $\eta$ describes the viscosity of the polymer and $\Phi$ is the volume fraction of the filler. Later on, Guth and Gold modified this equation to take particle-particle interactions at higher filler loadings into account [29]:

$$
\eta_{\Phi}=\eta \cdot\left(1+2.5 \Phi+14.1 \Phi^{2}\right)
$$

Smallwood modified this equation by replacing the viscosity with the shear moduli in case of elastic materials [30]: 


$$
G_{\Phi}=G \cdot\left(1+2.5 \Phi+14.1 \Phi^{2}\right)
$$

Where $G_{\phi}$ is the shear modulus of the filled and $G$ is the shear modulus of the unfilled system. This equation is only valid for spherical particles. Therefore, Guth introduced the shape factor $\mathrm{f}$, which is the ratio of the longest and shortest diameter of the particle, to also take non-spherical particles into consideration [31]:

$$
G_{\Phi}=G \cdot\left(1+0.67 f \Phi+1.62 f^{2} \Phi^{2}\right)
$$

It was shown that this equation is able to describe the moduli of carbon black filled compounds up to a volume fraction of the filler $\Phi$ of ca. 0.22 [32].

\section{Occluded Rubber}

Medalia first claimed that the reinforcing effect is not only dependent on the volume fraction (hydrodynamic reinforcement) but also on the structure of the active filler. He proposed the idea of rubber being trapped inside the voids of carbon black. This occluded rubber does not participate in the elastic behavior at small strains and increases therefore the effective filler content. With increased stress and strain the filler agglomerates break up and the trapped rubber is released. As a result, the effective filler content decreases at higher strains. Hence, Medalia replaced the volume fraction of the filler $\Phi$ in equation 2.3 by the effective filler content $\Phi_{\text {eff }}[1,33-34]$ :

$$
\Phi_{\text {eff }}=\Phi \cdot\left(\frac{1+0.02139 \cdot D B P}{1.46}\right)
$$

Where $\Phi$ is the filler content and $D B P$ is the structure of the filler measured by the absorption of dibutylphthalate [35].

The combination of the hydrodynamic effect and occluded rubber theory (volume fraction and structure of fillers) still does not take any polymer-filler interactions into account. Therefore, Smit [36] and Pliskin [37] extended Medalia's approach by including a term to take the specific surface area of a filler into consideration as well. It is claimed that parts of the polymer segments are adsorbed at the surface of the filler (shell rubber) and therefore hindered in their mobility. Hence, they are partly excluded from a deformation during a mechanical stress and increase the effective volume fraction of the filler. Fig. 2.7 depicts the effect of the occluded rubber (structure) and shell rubber (surface area) on the volume fraction of fillers.
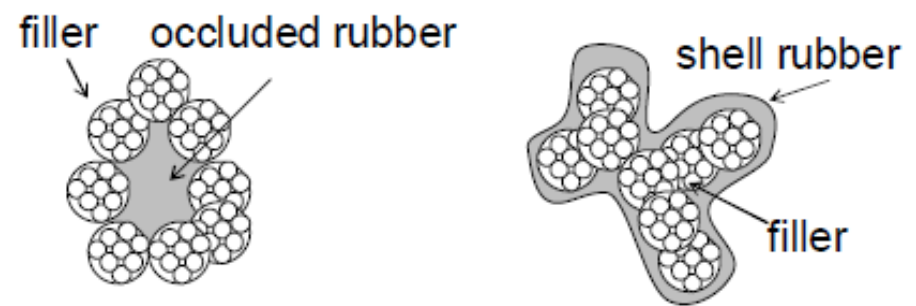

Figure 2.7: Increase in the volume fraction of a filler by the occluded rubber (lefthand side) and by the shell rubber (righthand side) [38]

\section{Filler Network - PAYNE-Effect}

Previously mentioned reinforcing model only takes the polymer-filler interactions into account. With increase of the filler concentration in the rubber the percolation threshold can be reached where filler-filler interactions play a decisive role forming a continuous filler network throughout the rubber matrix. This network results in a significant increase in stiffness and modulus of the compound. Payne initially described this effect which gives a strain-dependent contribution to the shear modulus. Fig. 2.8 depicts the shear modulus $G^{*}$ as a function of strain during a dynamic deformation. With an increase of the strain physical filler-filler interactions (e.g. Vander-Waals interactions) continuously break down in a non-linear way resulting in a drop of $G^{*}$. 
Simultaneous, rubber which is trapped inside the filler network (occluded rubber) is released and can participate in the deformation [39-40].

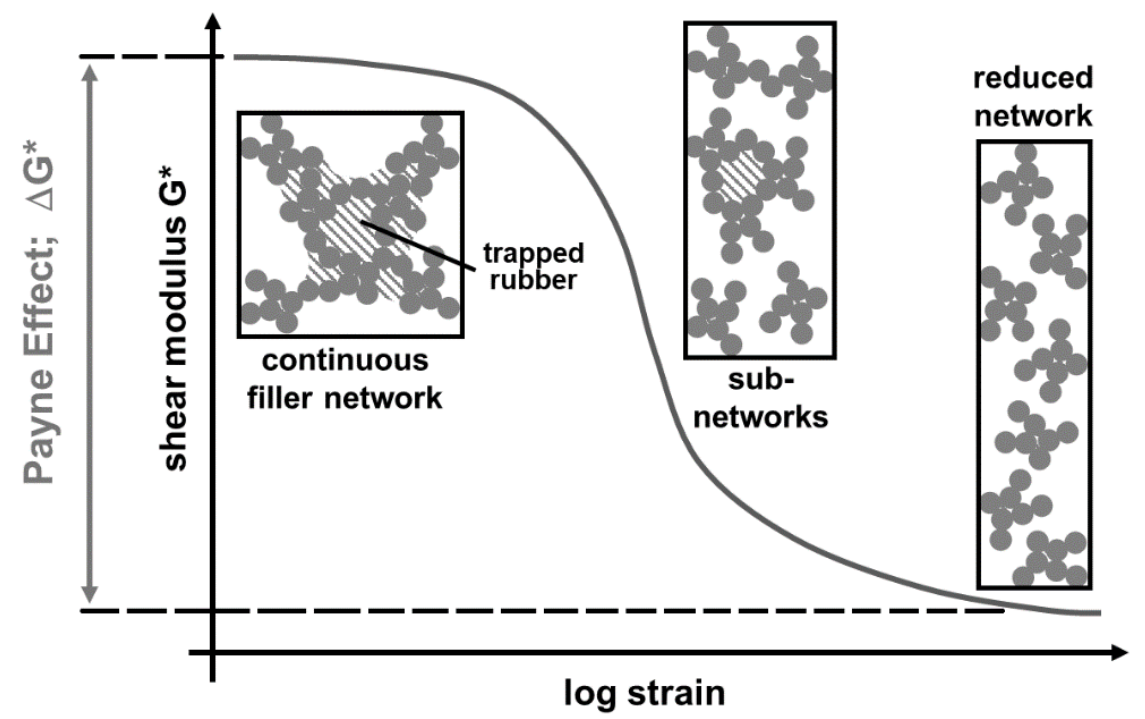

Figure 2.8: Break down of the filler network in dependency of the strain - PAYNE Effect [10]

The difference between the modulus at low strains $G_{0}{ }^{*}$ and the modulus at very high strains $G_{\infty}{ }^{*}$ is referred to as PAYNE effect $\Delta \boldsymbol{G}^{*}$ [39]. This effect is assumed to be reversible when the strain is released, provided that there is enough time for reagglomeration, and it is independent of the type of polymer. However, this effect is highly dependent on the type of filler as explained in a more detailed way by Donnet [41] who summarized all contributions to the rubber reinforcement for carbon black and silica filled compounds as additive effects (Fig. 2.9).

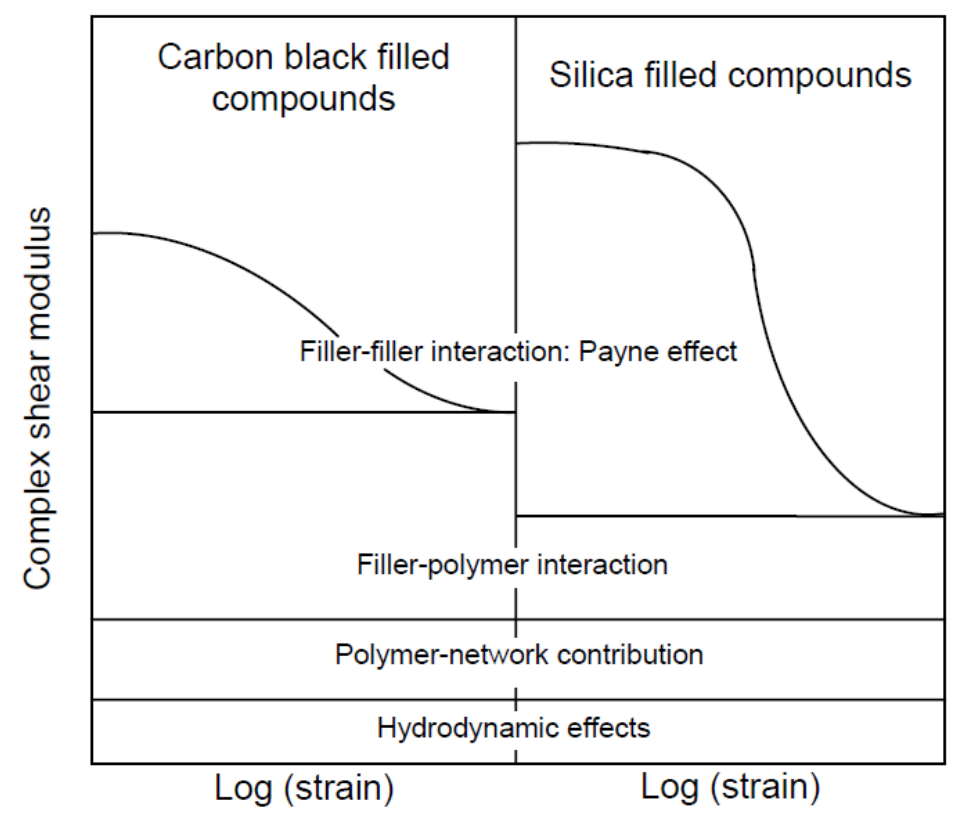

Figure 2.9: Additive effects of carbon black and silica on the shear modulus as a function of strain [41]

The filler-filler interactions respectively the PAYNE-effect represents the only strain dependent contribution. As can be seen in Fig. 2.9, the PAYNE-effect for silica filled compounds is much higher in comparison to carbon black reinforced rubber. This can be explained by the fact that silica has a high polar surface and surface energy. The formation of a strong silicasilica network via hydrogen bonds leads to a high viscosity respectively stiffness and modulus. The higher the surface area of silica, the higher the PAYNE-effect [42-43]. These higher filler- 
filler interactions compared to carbon black result in a higher PAYNE-effect [44]. A higher silica loading leads to a higher PAYNE-effect as well [45].

The strain independent additive effects can be divided in three contributions. The first one is the hydrodynamic effect as mentioned before which is mainly influenced by the volume fraction of the filler.

The polymer-network occurs due to the crosslinking of polymer chains. This network can be seen as well as a strain independent reinforcing effect. This network is build-up during vulcanization forming chemical connections between polymer chains. This results in an increase in hardness respectively modulus $G_{0}$ and can be calculated in accordance with the fundamental theory of rubber elasticity by equation 2.6 :

$$
G_{0}=v \cdot k_{B} \cdot T
$$

Where $v$ is the concentration of elastically active network chains, $k_{B}$ is the Boltzmann constant and $T$ is the temperature [1].

The third strain independent contribution are the polymer-filler interactions respectively inrubber structure. As depicted in Fig. 2.4 this contribution is dependent on the type of filler. The in-rubber structure of carbon black is a combination of polymer-filler interactions and the inter-aggregate structure via physical interactions described by the Oil Absorption Number OAN [35] which can be related to the occluded rubber as characterized beforehand. In contrast, silica does not show an in-rubber structure. On the one hand, its filler-rubber interactions are comparable weak due to the fact that silica is highly polar whereas rubber usually has a non-polar nature, which results in a low compatibility. On the other hand, investigations have proven [45] that the inter-aggregate structure of silica, measured e.g. by the DOA number [20], does not have an effect on the in-rubber structure. The higher filler-filler and lower filler-polymer interactions of silica causes a high viscosity and therefore a more complicated processing. Simultaneously, the dynamical-mechanical properties are reduced in comparison to carbon black filled compounds [44]. To achieve a better compatibility between silica and rubber, to reduce the filler-filler interactions and consequently to improve the level of reinforcement, coupling agents respectively silanes were introduced, which is described in the following.

\section{Silica-Silane System}

Nowadays, unmodified silica is only used in special applications like shoe soles and adhesive compounds due to bad compatibility between the filler and nonpolar rubbers as well as a difficult processing [7]. More established is the use of silica in combination with a silane. In general two types of silanes can be distinguished, monofunctional and bifunctional ones. Monofunctional silanes chemically couple to the polar surface of the silica during the mixing process. Therefore, filler-filler interactions are reduced by the shielding effect of the silane which lowers the viscosity of the compound and improves its processibility. However, the filler-polymer interactions are hardly improved by monofunctional silanes. To enhance the silica-rubber interactions and hence achieve a higher reinforcement bifunctional silanes were developed. The principle structure of a bifunctional silane is shown in Fig. 2.10: 


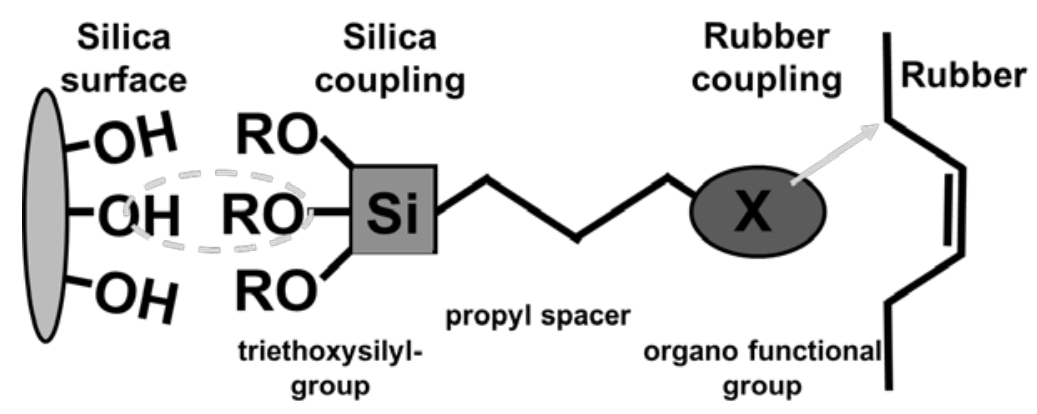

Figure 2.10: General structure of a bifunctional silane with a silica and rubber reactive side

A bifunctional silane consists of three different parts. The silica-reactive side (alkoxysilyl respectively triethoxysilyl group) chemically couples to the silica surface during mixing. The rubber-active side (organo functional group) reacts with the rubber during vulcanisation forming a chemical bond. Between both active sides is a hydrocarbon spacer which forms a flexible linkage to silica and rubber. In contrast to carbon black filled compounds, where the filler interacts with the polymer on a physical basis, the silica-silane filler system is chemically crosslinked to the rubber resulting in a high level of reinforcement as well [42, 46-47].

Several different types of silanes are applicable depending on the type of rubber and the acceleration system which is used. The most common ones used in the rubber industry are the sulfur-functional organosilanes bis-(triethoxysilyl-propyl)tetrasulfide (TESPT) and bis-(triethoxysilyl-propyl)disulfide (TESPD) which are applicable for sulfur-cured rubber compounds. Fig. 2.11 depicts the structure of TESPD as an example [10].

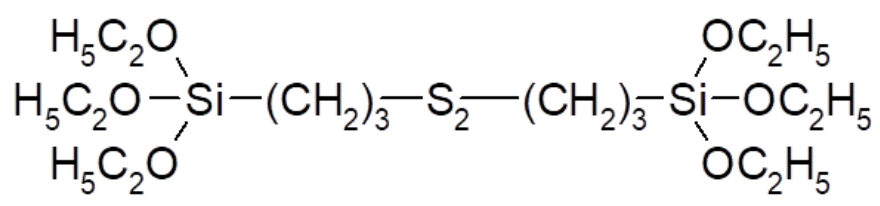

Figure 2.11: Structure of bis-(triethoxysilyl-propyl)disulfide (TESPD)

\section{Silica-Silane Reaction}

The silica-silane coupling, also referred to as silanization reaction, takes place during the mixing of the compound. This chemical reaction has to be controlled precisely due to the influence of the mixing temperature, moisture content as well as formation of ethanol. TESPD contains in average 2 sulfur atoms per silane. Therefore, less free sulfur is released during the silanization reaction and hence the risk of pre-scorch which means an early coupling of the silane to the rubber during mixing is reduced in comparison to bis-(triethoxysilyl-propyl)tetrasulfide (TESPT, $\mathrm{S}=\mathrm{S}_{4}$ ). However, more free sulfur needs to be added later on to optimize the silicarubber coupling and vulcanization process [7, 45]. Fig. 2.12 shows a simplified scheme of the coupling reaction of silica with silane. 


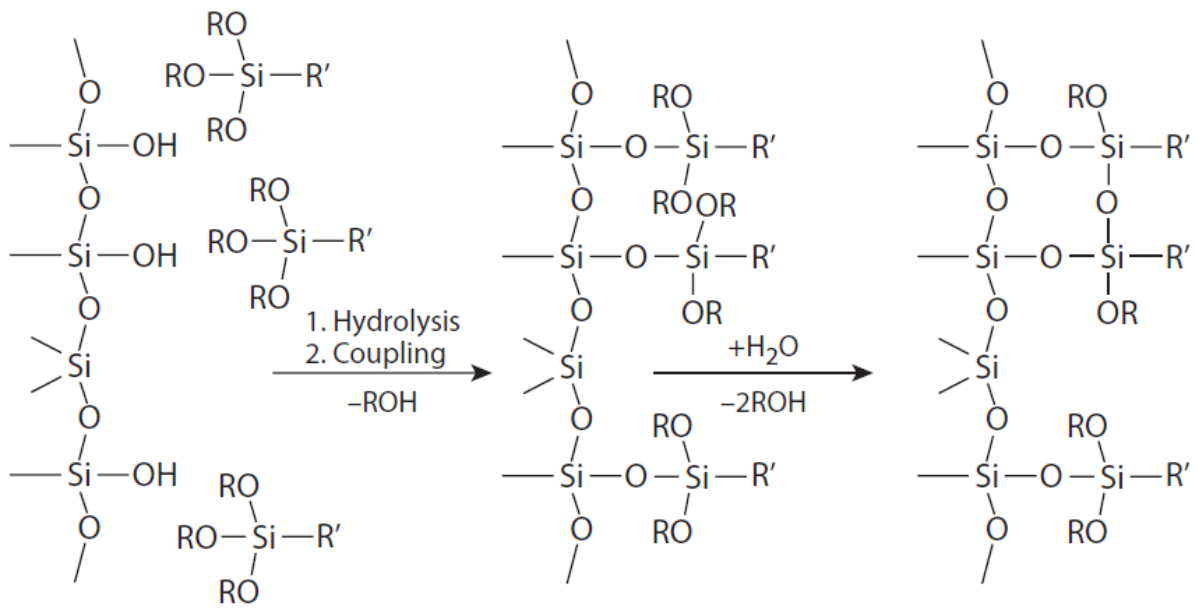

Primary reaction

Secondary reaction

Figure 2.12: Simplified reaction of silica with silane [10]

This reaction can in principle be divided into two parts, the primary and the secondary reaction. It is assumed that in first place one alkoxy group (silane) is hydrolyzed in the presence of water forming a higher reactive silanol group while alcohol is released. Subsequently, the activated silane couples to the silica surface forming a siloxane bond (primary reaction). During a second step (secondary reaction) neighboring silanes which already coupled to the silica can react due to an intermolecular condensation reaction forming siloxane bonds as well, assuming that the alkoxy groups were hydrolyzed previously [48-50]. Latest results indicate that in the Fig. 2.12 proposed secondary reaction cannot occur in this way due to steric hindrance [51].

\section{Silane-Rubber Reaction}

The silane rubber coupling ideally only takes place during the vulcanisation of the rubber compound. The chemical coupling of the rubber active side of the silane to the polymer in case of TESPD can exclusively occur in the presence of free sulfur and accelerators. The incorporation of sulfur by the silane leads to a formation of a sulfur bridge (at least 2 sulfur atoms) between a diene rubber and the silane itself [52-53]. A variety of investigations on the silanization reaction, reaction mechanisms and kinetics were performed in the last years. One worth mentioning is the work of Sato [54] who investigated the different reinforcing mechanisms of sulfide- and mercapto-silanes.

It can be stated that by means of the use of bifunctional organosilanes it is possible to form a chemical connection between the silica and the rubber (silica - silane - polymer) which leads to a significant increase in the compound reinforcement [47].

To summarize the concept of the filler network it can be said that the modulus of a reinforced compound at small amplitudes is mainly influenced by the filler-filler interactions whereas the modulus at larger deformations is highly dependent on the in-rubber structure of the filler. Besides the before mentioned theories there are other structure models to describe the reinforcing effect:

Kraus proposed in the Dynamic Network Model by [55] a model expanding the PAYNE-effect. $\mathrm{He}$ assumed that filler-filler contacts of carbon black agglomerates constantly break and reassemble during a strain sweep. The higher the deflection, the more pronounced the breakage. By means of the Dynamical Network Model the breakage and reagglomeration rate as well as the moduli can be calculated.

The Cluster-Cluster-Aggregate (CCA) Model by Heinrich and Klüppel [56] is based on the dynamic network model and was further developed to the Dynamic Flocculation Model (DFM) [57]. It is claimed that stiff filler clusters containing virgin bonds break with increasing strain. 
When the deformation decreases these broken clusters can re-agglomerate forming soft clusters containing damaged bonds. During a following strain these soft clusters can behave more elastically and store energy until they break again and dissipate energy.

\section{Adhesion models to describe the reinforcing effect}

All adhesion models are based on the assumption that the reinforcing effect is caused by interactions between polymer chains and the surface of a filler. These interaction can be divided in stable and non-stable ones during mechanical deformation. Stable interactions can be e.g. chemical bonds between the surface of the filler and the polymer chains whereas non-stable interactions are e.g. physical van der Waals forces. The most common adhesion models are briefly described in the following.

The first adhesion model to describe the reinforcing effect was developed by Twiss [58], Kraus [59] and Donnet [60] and is named bound rubber. It is assumed that polymer chains are adsorbed at the surface of a filler, hence hindered in their mobility and causing a mechanical reinforcement. The bound rubber content can be measured by extracting the polymer of a green compound from the filler by means of a suitable solvent. The part of the rubber which cannot be extracted is referred to as bound rubber content. Various investigations, summarized by Kraus [59], showed that this measurement method is strongly dependent on the measurement conditions, e.g. temperature, storage time, mixing time and the addition time of ingredients. Bound rubber is divided into chemically and physically bonded parts. The total amount of bound rubber is related to the microstructure of the polymer as well as the structure, chemistry and surface energy of the filler as shown by Wolff and Wang [61].

Funt [62] presented an idea of the Adhesion Model where he claimed that the reinforcement of rubber is controlled by two mechanisms: The hydrodynamic interaction as described beforehands and the chain entanglements. He suggested that rubber exists in three different states, bound rubber at the surface of the filler, bulk rubber in the matrix and a layer in between both rubber states called the transition zone. Entanglements formed in this transition zone lead to an increase in the effective crosslinking density and therefore a change in the properties, especially at low strains. Polymer chains being absorbed at the surface of the silica can be released at higher strains causing a decrease in e.g. the moduli.

Maier and Göritz proposed the kinetic model of the variable network density. Polymer chains are partly adsorbed at the surface of the filler. Depending on the degree of absorption of the rubber segments the polymer-filler interactions can be divided into stable and unstable contacts. When the strain respectively the amplitude is increased, the polymer chains with the least contact to the filler surface desorb at first. This results in a drop of the moduli. It is assumed that the moduli are directly proportional to the amount of polymer-filler contacts and that other influences are neglected [63].

The concept of the immobilized layer by Berriot et al. [64] and Wrana and Härtel [65] distinguishes between 3 different types of polymer shells which surrounds the surface of a filler:

- the glassy state is in direct contact with the filler, is fully restricted in its movement and therefore has a high modulus

- a second layer is located on top of the glassy state which has a slightly restricted mobility

- the outer layer of the polymer shell is highly flexible and is stretched under deformation

The immobilized layer has a high modulus in comparison to the polymer matrix and depends on the temperature. The lower the temperature, the more restricted the immobilized layer in its mobility. This leads to a thicker polymer shell and therefore reduces the distance between the filler particles which result in a higher filler-filler interaction respectively filler networking as depicted in Fig. 2.13. 


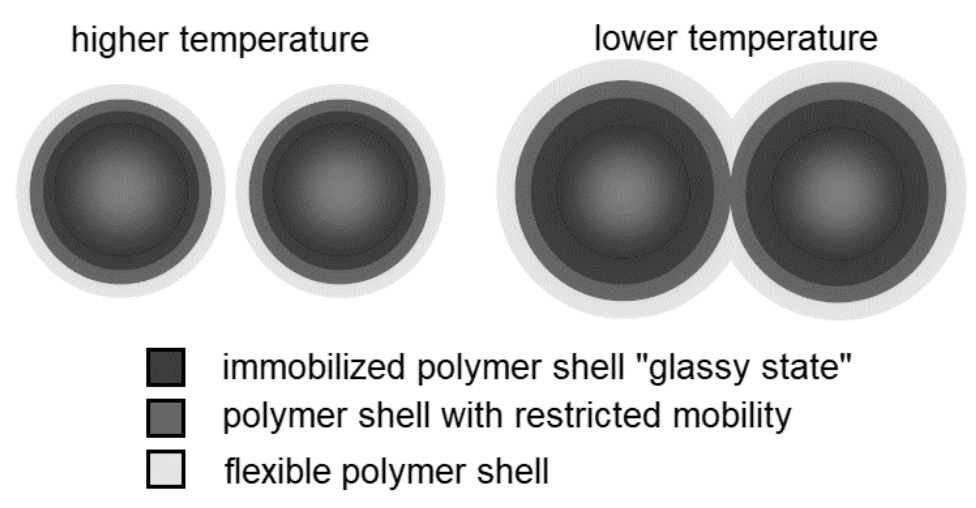

Figure 2.13: Schematic view of the immobilized layers at higher and lower temperatures [66]

During an external deformation two different effects occur. On the one hand filler-filler and fillerpolymer contacts are detached. On the other hand the immobilized layer between two clusters is deformed. A higher distance between two particles leads to a decrease in the modulus and therefore to a softening of the whole system. By means of the immobilized layer model the frequency dependence of the moduli at high amplitudes can be explained [64-65].

\subsubsection{Dynamic mechanical properties of elastomers and their effect on tire properties}

The performance of a tire can be characterized by various characteristics. The three most important parameters are the rolling resistance, the wet traction and the abrasion resistance. These properties depend on the compound of the tire and are strongly influenced by the dynamic mechanical properties of the rubber. The relationship between the tire performance and the dynamic mechanical properties of the compound can be explained by the viscoelastic behavior of the rubber. Due to an energy input the rubber is deformed. This energy is partly stored elastically inside the material and partly dissipated in form of heat also known as hysteresis. The viscoelastic behavior of a material can be modeled using a sinusoidal shear deformation $\gamma(t)$ of an angular frequency $\omega$ where the shear stress response $\sigma(t)$ is phaseshifted $[44,67]$.

$$
\begin{aligned}
& \gamma(t)=\gamma_{0} \sin (\omega t) \\
& \sigma(t)=\sigma_{0} \sin (\omega t+\delta) \\
& \sigma(t)=\left(\sigma_{0} \cos \delta\right) \sin \omega t+\left(\sigma_{0} \sin \delta\right) \cos \omega t
\end{aligned}
$$

$Y_{0}$ is the maximum strain, $t$ is the time, $\delta$ is the phase angle and $\sigma_{0}$ is the maximum stress. This delay and the resulting phase-shift are depicted in Fig. 2.14.

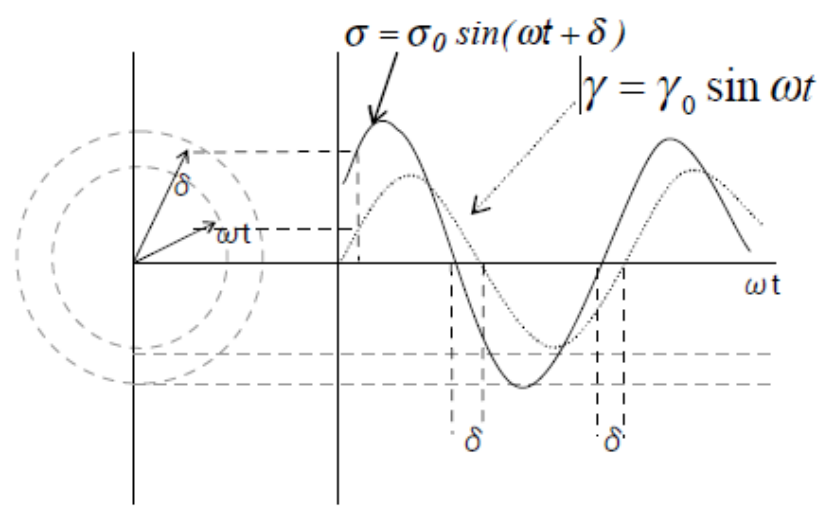

Figure 2.14: Sinusoidal stress deformation and delayed shear stress response resulting in a phase-shift respectively phase angle between 0 and 90 [68]

The shear stress signal consists of two contributions, the storage or elastic modulus $G$ ' which is in phase and the loss or viscous modulus $G$ " which is $90^{\circ}$ out of phase. 


$$
\begin{gathered}
\sigma(t)=\gamma_{0}\left(G^{\prime} \sin \omega t+G^{\prime \prime} \cos \omega t\right) \\
G^{\prime}=\frac{\sigma_{0}}{\gamma_{0}} \cos \delta \\
G^{\prime \prime}=\frac{\sigma_{0}}{\gamma_{0}} \sin \delta
\end{gathered}
$$

On the basis of the equations $2.7-2.12$ the shear modulus $G^{*}$ can be written in a complex form. Thereby, the storage modulus $G^{\prime}$ can be seen as the real part and the loss modulus $G$ " represents the imaginary part of $G^{*}$.

$$
G^{*}=G^{\prime}+i G^{\prime \prime}
$$

The ratio of the loss modulus to the storage modulus finally results in the phase angle or loss factor tangent $\delta$ as shown in equation 2.14:

$$
\tan \delta=\frac{G^{\prime \prime}}{G^{\prime}}
$$

The moduli G' and G" are frequency dependent which is related to the segment and chain mobility of the elastomer. Fig. 2.15 depicts both moduli as a function of the frequency. The plot shows four different frequency zones as well as two areas in which rolling resistance and wet traction play a decisive role.

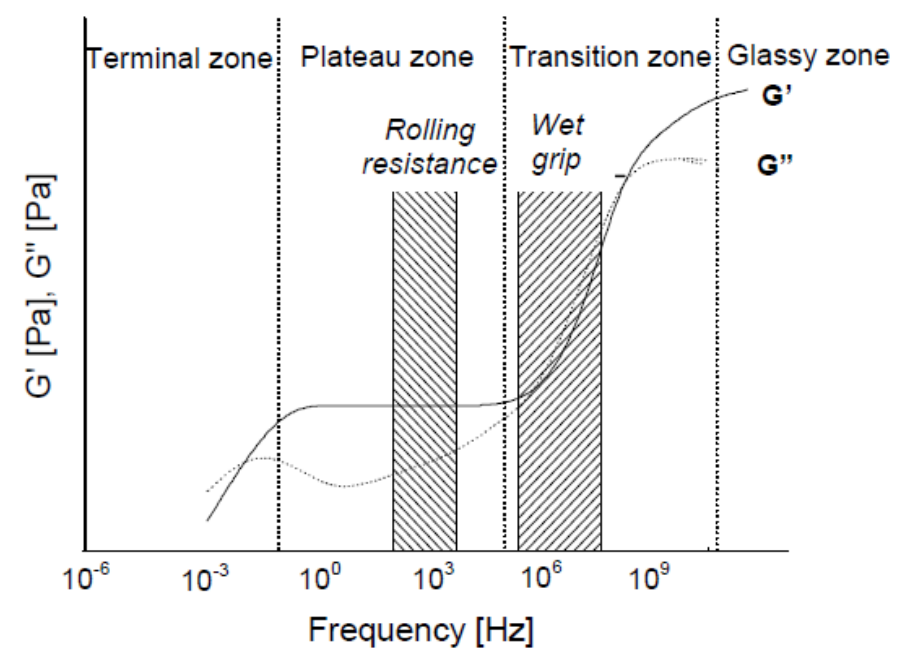

Figure 2.15: Frequency dependence of G' and G" [69]

In the terminal zone at low frequencies the polymer chains and segments are able to follow the applied strain without any delay respectively loss of energy. When increasing the frequency to a point where entanglements are no longer capable of following the applied strain they will act temporarily as crosslinks. In this area, the storage modulus shows a plateau whereas the loss modulus reaches a minimum. The third region is referred to the transition zone where both moduli show a pronounced increase due to a decreasing mobility. In the final zone at very high frequencies polymer chains and segments can hardly react to the applied strain, behave rigid and show a very high modulus [69].

The rolling resistance of a car tire is defined as the force in the opposite direction of the motion which has to overcome in order to move the car. This energy loss known as hysteresis is related to the loss factor tan $\delta$ at lower frequencies $\left(10^{2}-10^{4} \mathrm{~Hz}\right)$ in accordance with the angular frequency of the tire [70]. On the contrary, wet skid resistance is a high frequency phenomena in the megahertz region. Skidding appears during a braking process on wet ground which is called the lubrication effect or aquaplaning, where the tire slides over the surface of 
the road. Due to a high amount of small irregularities of the asphalt the tread is exposed to a high frequency of deformation in a short period of time $[67,71]$.

The frequency dependence of the dynamic mechanical properties of rubber compounds is a consequence of the change in the mobility of the polymer chain and its segments. Therefore, the moduli are as well temperature dependent. The concept of the temperature-frequency equivalence was initially described by Williams, Landel and Ferry. They established the concept of the time-temperature superposition also known as WLF-model. It is claimed that a polymer behaves similar at high frequencies respectively at low temperatures and similar at low frequencies respectively high temperatures [72]. Fig. 2.16 shows the storage, loss modulus and loss angle as a function of the temperature.

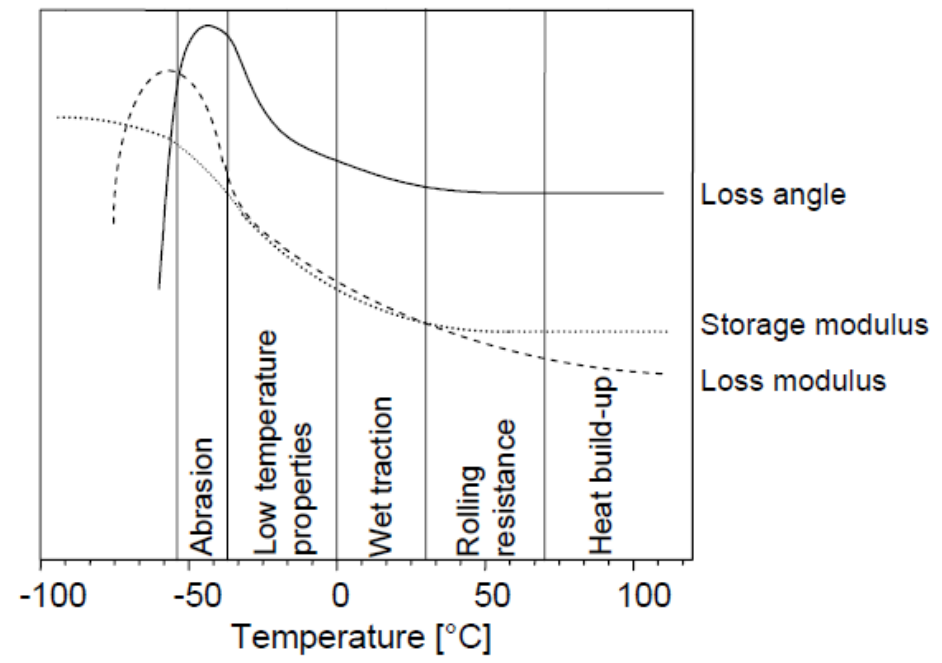

Figure 2.16: Temperature dependency of G', G" and tand and their effect on tire properties [68]

The low temperature area including the glass transition point (peak of the storage modulus curve) corresponds to the glassy zone of Fig. 2.15. Heinrich [73] claimed that the glass transition temperature of a compound correlates to the abrasion resistance of a tire. At temperatures around the freezing point the low temperature properties and the wet traction play a decisive role. The higher the loss modulus at $0{ }^{\circ} \mathrm{C}$, the higher the expected wet skid resistance. However, these dependencies of temperature ranges with tire performances can only be seen as indications. The temperature range around $60^{\circ} \mathrm{C}$ corresponds to the temperature of the tire during use where the rolling resistance is decisive. The lower the loss modulus respectively the rolling resistance, the better the tire performance. At even higher temperatures the material starts to decompose and safety is reduced. Therefore, the heat build-up should be as low as possible to prevent an untimely destruction of the tire [73].

In addition, the dynamical mechanical properties of elastomers are also dependent on the crosslinking density and structure as e.g. investigated by Bandzierz et al. [74-75]. Finally, the type of filler which is used has to be taken into account. Fig. 2.17 depicts the loss angle as a function of the temperature for a carbon black and silica filled compound. 


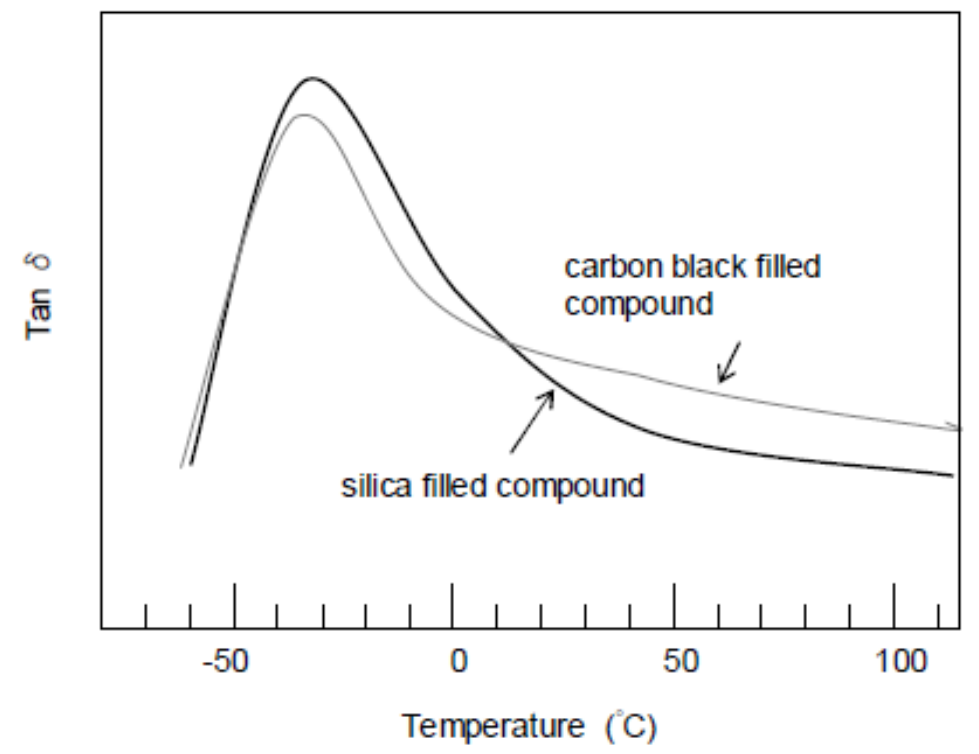

Figure 2.17: Temperature dependency of tan $\delta$ for silica and carbon black reinforced rubber $[44,68]$

The major difference between both types of fillers can be seen in the area between 10 to $80^{\circ} \mathrm{C}$. This temperature range corresponds to the rolling resistance of a car tire as discussed beforehand. By replacing carbon black with silica the hysteresis is reduced which leads to a desired lower rolling resistance. A second minor advantage can be seen in the area of the glass transition temperature. A higher peak of the loss modulus of silica filled tread compounds indicates an improved wet traction. Both phenomena can be related to the different surface properties of both fillers, especially the filler-filler interactions respectively PAYNE-effect as well as the polymer filler interactions [44].

\subsection{Dispersion}

„... Dispersion must be known ... in conjunction with physical testing of experimental compounds, ..., since in each case failure to achieve good dispersion would mean that optimum properties had not been realized" [76].

\subsubsection{Definitions}

The term "dispersion" originally had the meaning of scattering (lat. dispersio, from dispergere "distribute, spread, dispel") and generally describes a fine distribution. The chemical definition of dispersion is a system in which fine particles of one substance are scattered in a continuous phase throughout another substance [77]. According to ASTM D 3053, dispersion is defined as the "degree of uniform distribution of a filler's primary unit (i.e., aggregate of carbon black) into a compound" [78]. In the following the term "dispersion quality" is used to describe the degree of size reduction of a filler inside a rubber matrix whereas "distribution" characterizes the homogeneity of the filler in the rubber matrix. The in-rubber dispersion quality can be distinguished between visual, macro- and micro-dispersion (Fig. 2.18). Visual dispersion $(>100 \mu \mathrm{m})$ can usually be detected by a human eye without any magnification. The term "macro-dispersion" describes the degree of a filler distribution at a scale of 2 up to $100 \mu \mathrm{m}$, whereas the degree of a filler distribution at a scale smaller than $2 \mu \mathrm{m}$ is referred to as microdispersion [78].

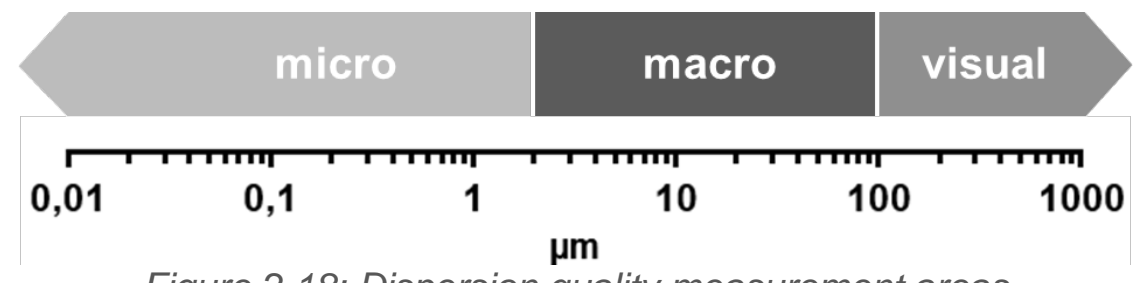

Figure 2.18: Dispersion quality measurement areas 
Compared to dispersion, the term "dispersibility" describes the relative ability of fillers to be dispersed and uniformly distributed in a rubber matrix. This approach is used in the present work. According to Stöckelhuber et al. [79] several thermodynamic parameters have to be taken into account to assess the dispersibility of fillers. In general, different types of one type of filler (e.g. silica) can only be assessed regarding their dispersibility within the same formulation, mixing equipment and mixing process.

\subsubsection{Dispersion Process}

The dispersion process of fillers in general occurs during the mixing process of the rubber compound inside an internal mixer. Various different models and mechanisms to describe this process were proposed in the past. In the following, only the most common and basic ones are summarized.

\section{Requirement for dispersion}

Bagster and Tomi [80] investigated the stress field around a spherical particle inside a counterrotating shear cell. This stress results in tension and compression and the general conditions for dispersion were stated as following:

$$
\sigma_{\text {hydrodyn }} \geq \sigma_{\text {cohesion }}
$$

where $\sigma_{\text {cohesion }}$ includes all intrinsic properties of clusters respectively particle-particle interactions and $\sigma_{\text {hydrodyn }}$ is defined as the shear stress in dependency of the viscosity $\eta$ and shear speed $\bar{y}$ as shown in equation 2.16:

$$
\boldsymbol{\sigma}_{\text {hydrodyn }}=\frac{\mathbf{5}}{2} * \boldsymbol{\eta} * \dot{\gamma}
$$

The dispersion process therefore can only occur when the shear stress is at least as high as the intrinsic forces to overcome particle-particle interactions.

\section{Steps of dispersion}

In 1965, Palmgren [81] proposed a basic model which divides the mixing process into four elementary steps (Fig. 2.19):

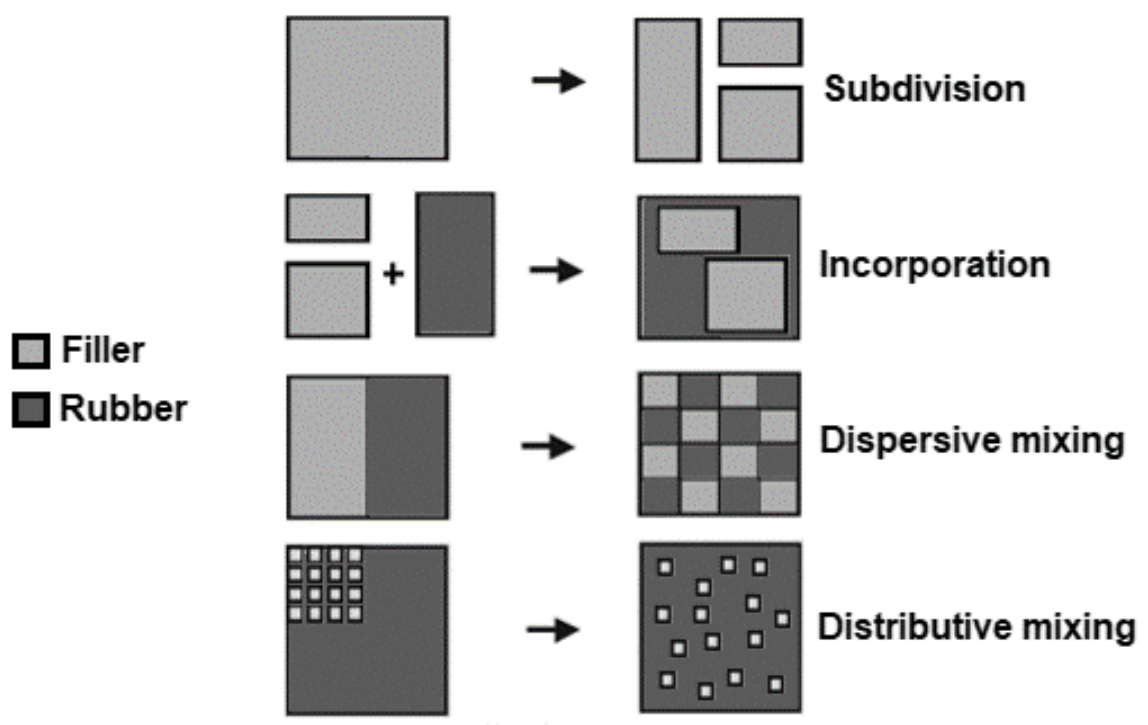

Figure 2.19: Filler in-rubber dispersion model

The first step that occurs at the beginning of the mixing is the subdivision of large clusters (e.g. granules) which break into smaller fragments. These clusters are able to be incorporated into the rubber matrix in the second step. Due to the structure of the filler, rubber can penetrate into the free voids of the filler clusters and replace the trapped air. During that process a coherent mass is formed and therefore energy from the mixer consumed to allow further steps. 
During the dispersion process, the size of agglomerates are reduced to smaller units, ideally into aggregates (ultimate size) by means of shear, tension and compression stress [82]. In addition, a distribution of the particles inside the rubber matrix takes place. The dispersion efficiency decreases with reduced viscosity during mixing and hence a minimum particle size is reached where a further energy input does not result in a further size reduction [83]. In the last mixing step the fillers (ideally aggregates) are distributed randomly inside the rubber matrix to achieve a homogeneous compound. During the distributive mixing, the physical shape of the filler particles remains unchanged. In general, dispersive and distributive mixing occur at the same time.

\section{Dispersion mechanisms}

Typical mechanisms to describe the dispersion process of fillers are the rupture and erosion models. Collin [84] and Peuvrel-Disdier [85] investigated the different dispersion mechanisms for carbon black and silica as well as the differences between granules and micro-pearls. It was stated that silica in general needs a higher critical shear stress to be dispersed in comparison to carbon black due to higher intrinsic properties of the agglomerates respectively cohesion forces. Moreover, granules are mainly reduced in size by rupture into several large fragments during the first dispersion step whereas micro-pearls undergo another mechanism named disintegration where a large number of smaller fragments are created within a short period of time. Fig. 2.20 depicts the three different dispersion mechanisms presented by the example of a micro-pearl.

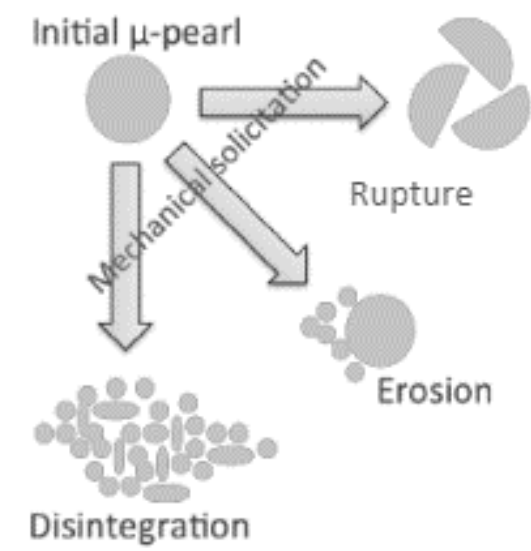

Figure 2.20: Filler in-rubber dispersion model [86]

It was claimed that rupture is a mechanism which is very efficient at the beginning of the dispersion process and independent of the intrinsic parameters but limited to a critical minimum size where it cannot occur anymore. It is a sudden mechanism which requires a critical stress resulting in a breakage. Erosion in turn is a slow continuous process crucial for later steps during the dispersion process and said to be very sensitive to the intrinsic parameters of the filler. It is a gradual detachment of aggregates based on the model of Kao and Mason [87].

\subsubsection{Dispersion Measurement Methods}

To determine the in-rubber dispersion quality of fillers several methods are available, either for measuring the macro- or the micro-dispersion. The most common techniques can be divided into mechanical, light optical and electrical as well as certain special techniques. Tab. 2.3 gives a general overview of different dispersion measurement methods and their respective resolution limits. 
Table 2.3: Dispersion measurement methods and their resolution limits

\begin{tabular}{lc}
\hline Dispersion Measurement Method & Resolution limit* in $\mathbf{\mu m}$ \\
\hline Mechanical & $2-15$ \\
EVONIK Topography Test & $0.005-1$ \\
Atomic Force Microscopy (AFM) & $>100$ \\
\hline Light Optical Reflection & $>10$ \\
ASTM D 2663 Method A & $4-42$ \\
PHILLIPS Method & $>0.250$ \\
Dispergrader/-tester & 0.83 \\
Dispersion Index Analysis System (DIAS) & $>5$ \\
Confocal light microscope (CLM) & not applicable for silica \\
CABOT TL & illed rubbers \\
\hline Electrical & $0.0005-1$ \\
\hline Special & $>0.025$ \\
Transmission Electron Microscopy (TEM) & $>1$ \\
Scanning Electron Microscopy (SEM) &
\end{tabular}

*depending on the used system and evaluation procedure

The visual dispersion is usually not taken into consideration when the dispersion quality of a rubber compound is measured. However, Abraham et al. investigated the influence of defects (respectively undispersed filler particles) to rubber properties up to $200 \mu \mathrm{m}$ in diameter. It was shown that the lifetime of a rubber product is decreased with increasing cluster size [88].

\section{Mechanical Dispersion Measurement Methods}

The mechanical - also termed tactile - measurement methods differ on two main aspects. The EVONIK Topography Test method measures the dispersion quality on a macro scale whereas the Atomic Force Microscopy method (AFM) quantifies the micro-dispersion of the filler in reinforced compounds. Both measurement methods were originally used in metallurgy, for example to detect the roughness of ceramic surfaces. The measured surface roughness of a rubber sample can be directly correlated with the filler distribution and dispersion quality. Influences that are due to variability in sample preparation (for example stripes from a damaged blade) can be recognized and excluded by suitable evaluation software [89].

\section{EVONIK Topography Test}

Based on ASTM D 2663 - Method C [90], macro-dispersion can be evaluated by applying the EVONIK Topography Test (Topo). This quantitative method is used to detect the surface roughness of rubber compounds independent of the type of filler used. A freshly cut rubber surface is required for the measurement. While cutting the rubber specimen with a razor blade, the softer polymer is divided into two halves whereas the harder filler particles remain intact only on one side of the cut rubber sample. Consequently, certain irregularities (such as protrusions and depressions) will be created as shown in Fig. 2.21-A. The resulting roughness can be scanned by a suitable probe. Fig. 2.21-B shows how the surface is scanned over a total area of $5 \mathrm{~mm}^{2}$ (100 profiles) by a stylus with a diamond tip of $5 \mu \mathrm{m}$ radius. The resulting lines assembled together create a spatially differentiated profile (Fig. 2.21-C), including information about peak heights and the defected area (Fig. 2.21-D) [89-91]. 
A
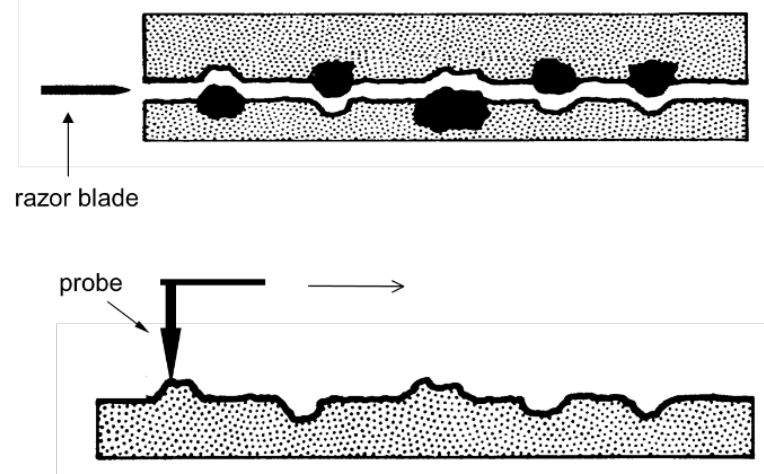

C

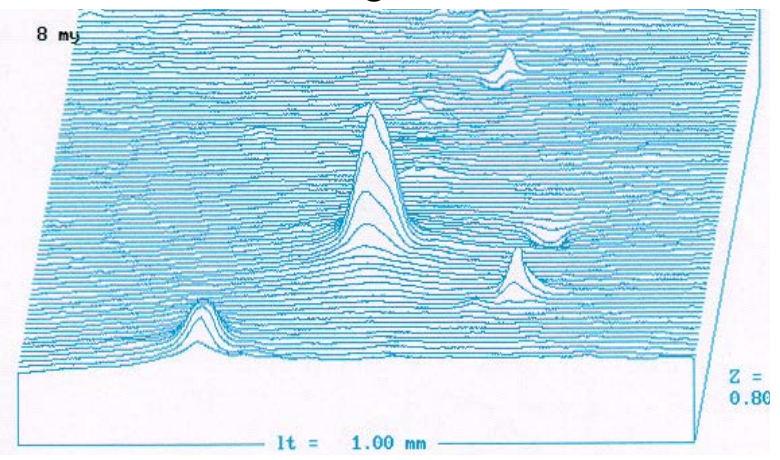

B

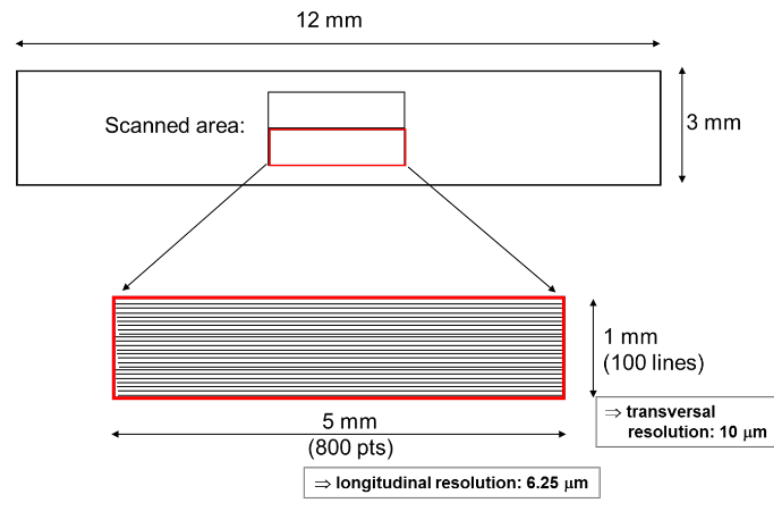

D

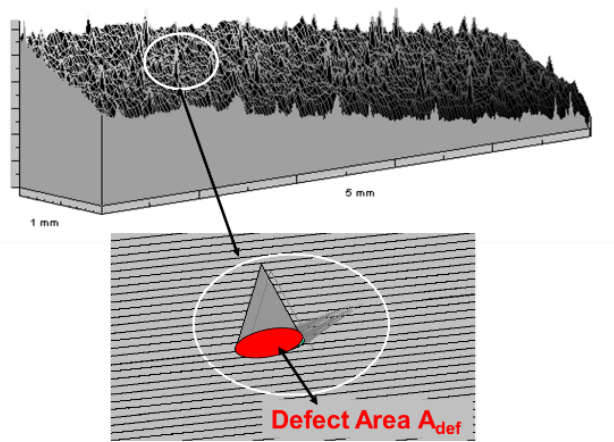

Figure 2.21: EVONIK Topography Test [91]

A computer software program calculates the roughness value, the total number of peaks, the average peak heights and the defected area. The resolution is limited to defects larger than 2 $\mu \mathrm{m}$ in diameter. The dispersion quality can be compared to light optical dispersion measurements and is shown visually by means of a photorealistic image (Fig. 2.22).

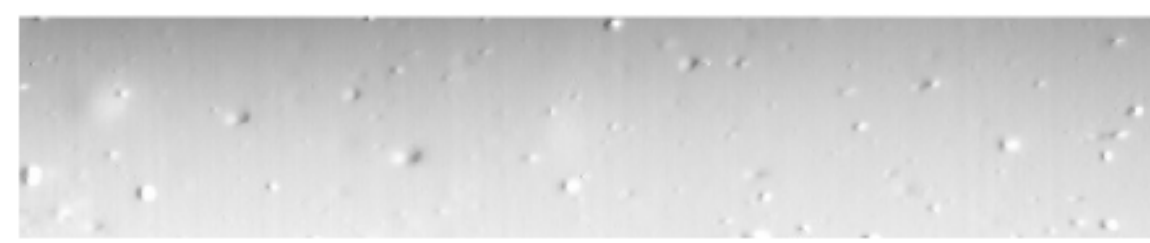

Figure 2.22: Visualised Topography measurement

\section{Atomic Force Microscopy (AFM)}

The Atomic Force Microscopy (AFM) method is used for mapping a sample surface, assessing its roughness, and is in principle comparable to the EVONIK Topography Test. However, this method can be transferred to a much smaller scale and is able to detect the micro-dispersion quality (> $5 \mathrm{~nm}$ ) of a rubber compound. The sample preparation can be done in the same way as for the Topography Test. The principle assembly of an AFM measurement system is depicted in Fig. 2.23. The ultrafine tip (10 to $100 \mathrm{~nm}$ ) is attached to a cantilever. A piezoelectric adjusting element is able to control the movement in three dimensions. A laser can detect even the smallest deflections of the probe [92]. 


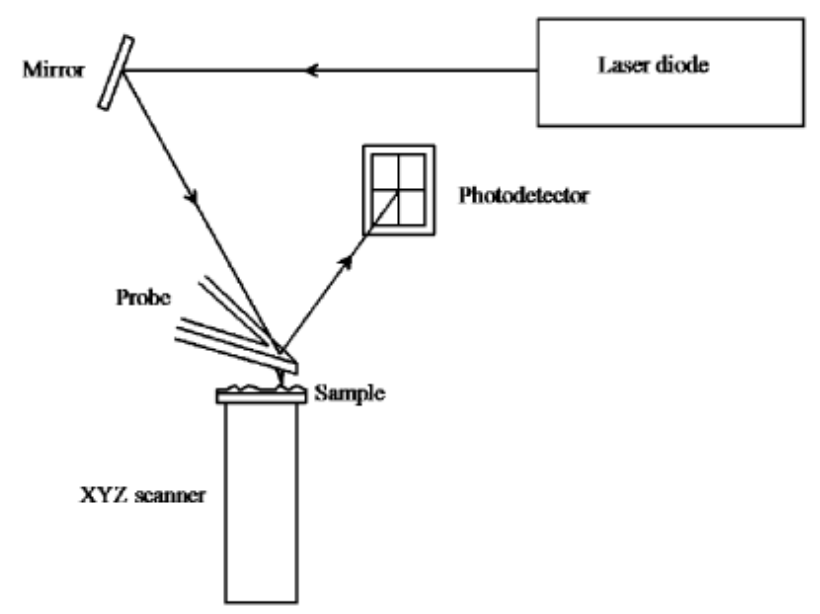

Figure 2.23: Principle assembly of AFM [93]

When the probe is close enough to the surface (but still not touching it), interactions between the tip and the sample surface occur, e.g. due to van-der-Waals forces. Because of differences between the chemistry of the filler and polymer it is possible to scan the surface in this noncontact mode. A second approach is the use of the contact mode whereby the surface is scanned in the $x$ - and $y$ - directions where the tip is in contact with the sample. Additionally, the roughness is acquired in z-direction from the height information. In a variation of the contact mode (lateral force or constant-height mode), the pressure force in z-direction is set to a fixed value and kept constant by an electronic feedback. A third alternative is the tapping mode. The tip oscillates with a certain amplitude (20 to $100 \mathrm{~nm})$ and frequency $(\sim 300 \mathrm{kHz})$ while striking the sample surface. Different disturbances on the oscillation amplitude due to surface variations are detected by a feedback system [93-94]. Johnson [93] summarized several additional modes for the AFM which can be used to investigate rubber e.g. to examine the microstructure of polymer blends.

One big advantage of the AFM method is that the distance between the tip and the surface can be varied step-by-step to only a few $\mathrm{nm}$. The results generated can be transferred to a three dimensional map with a high resolution of particle characteristics and dispersion (Fig. 2.24). However, unvulcanised rubber can only be investigated in contactless mode due to the fact that raw compounds are, in general, too sticky and soft for fine tactile measurements [95].

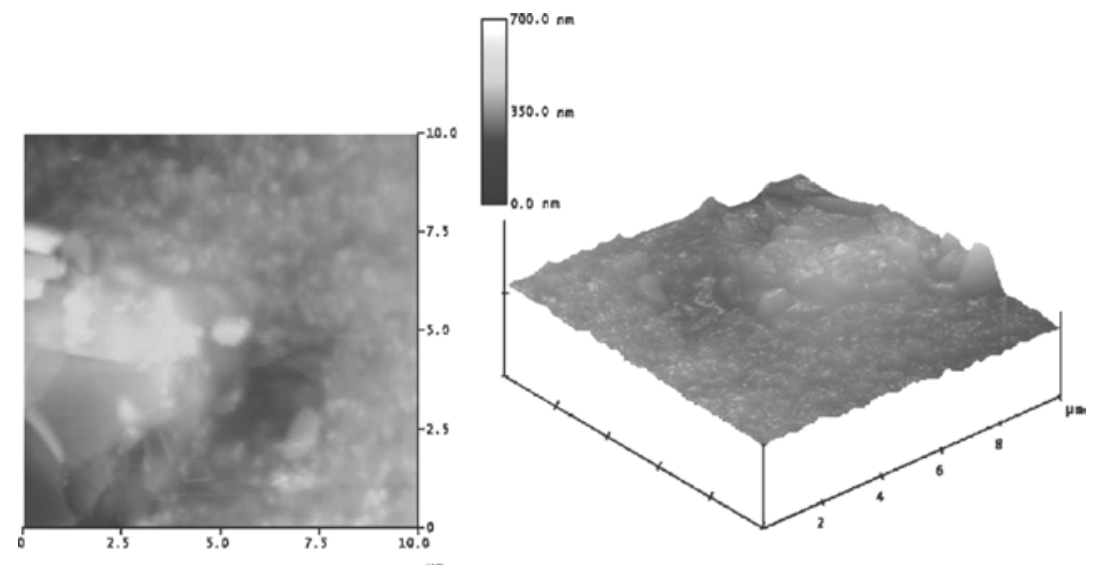

Figure 2.24: Example for a 2D and 3D AFM image [95]

\section{Light Optical Dispersion Measurement Methods}

The light optical dispersion measurement methods can be divided basically into two categories, namely the reflection and transmission method. The most common reflection methods are ASTM D 2663 method A, PHILLIPS, Dispergrader/-tester, the Dispersion Index Analysis 
System (DIAS) and the recently developed confocal laser microscope (CLM), whereas the typical transmission method is the CABOT TL (ASTM 2663 method B).

\section{Reflection Methods ASTM D 2663 Method A}

One of the first developed and simplest methods for a rough estimation of the dispersion quality is described in the ASTM D 2663, method A. Cured rubber samples or uncured pressed samples are cut using a sharp razor blade and the exposed fresh surface is examined visually under a magnifying glass or a binocular microscope with a maximum of $20 x$ magnification. The operator compares the visual dispersion to five standard pictures to determine the dispersion quality. This method is strongly dependent on the operator's experience and is, according to ASTM, only applicable for carbon black filled rubber. Therefore, it is rarely used nowadays [96].

\section{PHILLIPS Method}

The PHILLIPS method is a further development of the ASTM D 2663 method A. Fig. 2.25 depicts the PHILLIPS-scale for evaluating the macro-dispersion quality. It distinguishes between ten dispersion standard scales of a razor cut surface, in which "1" means a very poor and "10" a very good level of dispersion. The surface is evaluated at 30x magnification under an optical light microscope illuminated at a defined irradiation angle of $30^{\circ}$ [97].

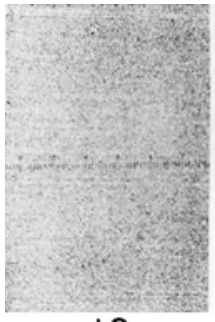

10

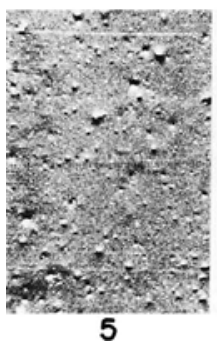

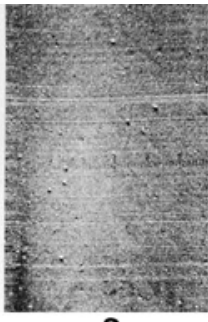

9

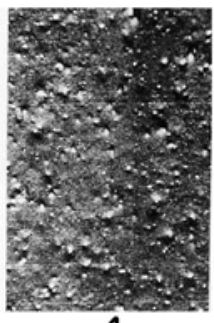

4

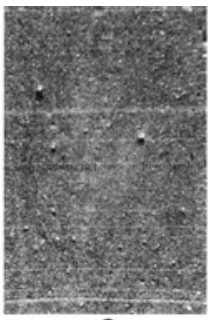

8

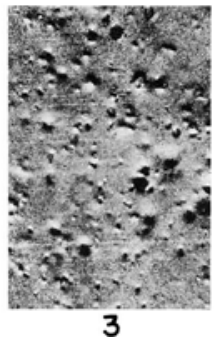

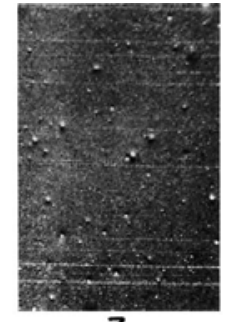

7

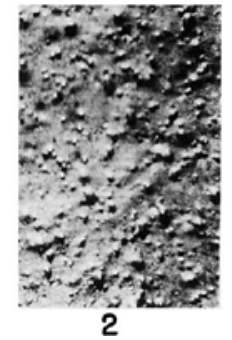

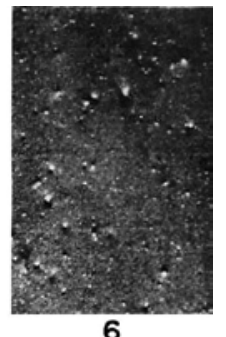

6

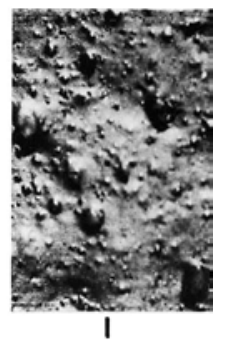

\section{Dispergraderl-tester}

The Dispergrader and Dispertester are related measurement systems based on ASTM D772311, the Standard Test Method for Rubber Property - Macro Dispersion of Fillers in Compounds. Similar to the PHILLIPS method, the cut rubber sample is investigated by an optical light microscopy with $30^{\circ}$ irradiation angle and usually at 100x magnification (Fig. 2.26). The Charge-Coupled Device (CCD) camera used digitises a grayscale image that can be automatically compared to standard scales with a rating from 1 to 10 for carbon black (for example the PHILLIPS scale), silica and silica/carbon black compounds to determine the dispersion quality. Furthermore, the percentage dispersion of fillers can be calculated by evaluating the white area (the scanned area that contains undispersed fillers) related to the total scanned sample area. Additionally, the particle size distribution of undispersed filler clusters can be analysed [98]. 


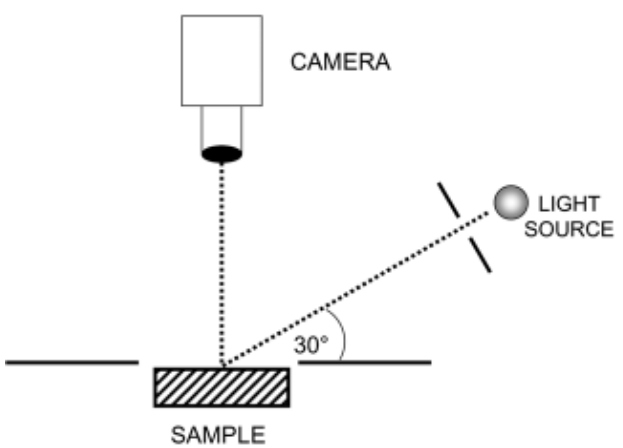

Figure 2.26: Schematic assembly of the Dispergrader/-tester system [99]

\section{Dispersion Index Analysis System (DIAS)}

The DIAS method is based on a typical reflected-light microscope in combination with suitable image analysis software and was developed by the German Institute of Rubber Technology. A razor-cut specimen surface is magnified 150x and investigated at 10 different spots having a total area of $2.76 \mathrm{~mm}^{2}$. Polymers reflect the incoming light whereas undispersed filler particles absorb it. The emerging difference in contrast is evaluated. As a result, the Dispersion Index (DI) according to Medalia [76] is determined. It indicates the percentage of filler being dispersed into the rubber matrix as a quantitative result provided that the filler volume is known. The higher the DI value (maximum of $100 \%$ ) the better the dispersion quality [100]. Eq. 2.17 describes the calculation of the dispersion index in detail:

$$
D I=100-\frac{(\Sigma \text { peak area }) * 10000 * \text { Medaliafactor }}{\text { filler volume } * \text { examined area }}
$$

where:

$$
\text { Medaliafactor }=\frac{\frac{\text { filler volume }}{100}+0.78}{2}
$$

and $\mathrm{DI}$ is the Dispersion Index in $\%$, filler volume in $\%, \Sigma$ peak area in $\mu \mathrm{m}^{2}$ and examined area in $\mu \mathrm{m}^{2}$.

\section{Confocal Light Microscope (CLM)}

Recently, Palm [101] and Kunkel [102] investigated the in-rubber dispersion quality of carbon black by means of the confocal light microscopy (CLM/CFM) method. The measuring principle is the following: A light beam passes a small pinhole, is then directed to the freshly cut rubber sample surface by a lens and reflected back towards the pinhole. As long as the surface is in the focus point of the beam (Fig. 2.27-a), the light passes the pinhole again and is recorded by a detector. In case that the beam is out of focus, the scattered light cannot pass the pinhole and therefore is not detected (Fig. 2.27-b). By scanning the surface with a fixed distance from the focal plate to the lens in z-direction, a two dimensional cross section is generated containing information about all spots which are in focus. By progressively changing the distance along the z-axis, multiple maps are obtained which are composed into a three-dimensional picture containing the height information respectively surface topography. 

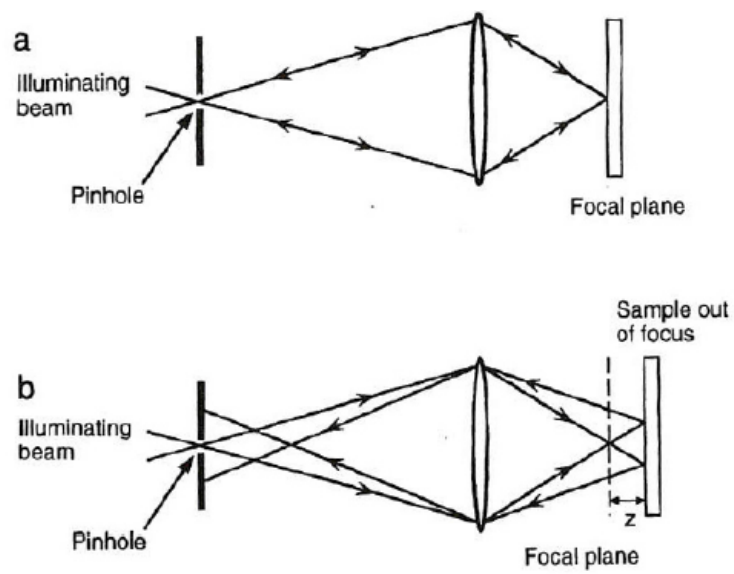

Figure 2.27: Schematic assembly of the CLM measurement system [101]

\section{Transmission Method}

The CABOT TL method is described in ASTM D 2663, method B (agglomerate count). It is a quantitative and direct measurement of the macro-dispersion. The vulcanised or unvulcanised rubber has to be frozen by liquid nitrogen and then cut by a glass blade or microtome to obtain a 1 to $2 \mu \mathrm{m}$ thin sample. Visible light shines partially through the rubber during the evaluation of the samples and is detected by a microscope having $75-100 x$ magnification. Filler particles are perceived as a shadow while the pure polymer is almost transparent. An area of $1 \mathrm{~cm}^{2}$ is evaluated. Ten samples are normally investigated for each compound to obtain a representative average. Inspired by the PHILLIPS scale, the measurement can either be compared to 48 different reference pictures summarized in the CABOT Dispersion Classification Chart or all agglomerates of larger than $5 \mu \mathrm{m}$ diameter can be analysed in terms of their number and size [103].

\section{Electrical Dispersion Measurement Methods}

The measurement of the electrical conductivity and resistance of a reinforced rubber sample is based on two general assumptions. Rubber is a very low or non-conductive material whereas the filler like carbon black is conductive. The better the micro-dispersion quality of a filler, the higher the resistivity of the whole rubber compound due to the fact that the particles being separated and do not form a conductive filler-filler network. Fig. 2.28 depicts the filler loading as a function of the compound volume resistivity [104].

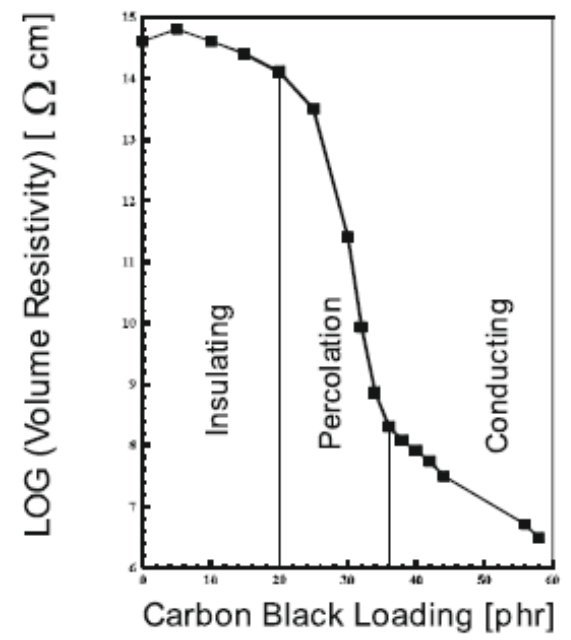

Figure 2.28: Electrical dispersion measurement [104]

In general, three different areas of the plot can be distinguished: the insulating, the percolation and the conductive zones. When the filler loading is below the percolation threshold and the particles are very well dispersed, no continuous filler-filler network is formed and therefore the 
resistivity is high. In the area of the percolation, a threshold current can pass through the sample and as a result the conductivity rises substantially. However, a further increase of filler loading does not affect the resistivity to a high degree because the filler-filler network is already fully build up [104]. The electrical dispersion measurement methods can be divided into two different classes: (1) the altering current (AC) method named impedance spectroscopy; and (2) the direct current (DC) method. However, conductive methods are very limited in their use and have only very limited applications. Especially the dispersion quality of non-conductive fillers (e.g. silica) cannot be determined with these techniques as well as compounds with very low, lower than $40 \mathrm{phr}$, very high filler loadings and depending on the particle size of the filler itself $[97,105]$.

\section{Special Dispersion Measurement Techniques \\ Transmission Electron Microscopy (TEM)}

One of the most commonly used measurement systems to determine the micro-dispersion of filled rubber compounds is the Transmission Electron Microscopy (TEM). An electron beam is bundled by means of a strong electric magnet and transmitted through a rubber sample. For this purpose, ultra-thin cryosections having a thickness of 50-150 nm of the rubber samples have to be prepared by ultramicrotome at very low temperature below the glass transition temperature of the compound. A screen detects the transmitted beam and converts the signal into a two-dimensional image (Fig. 2.29) [106].
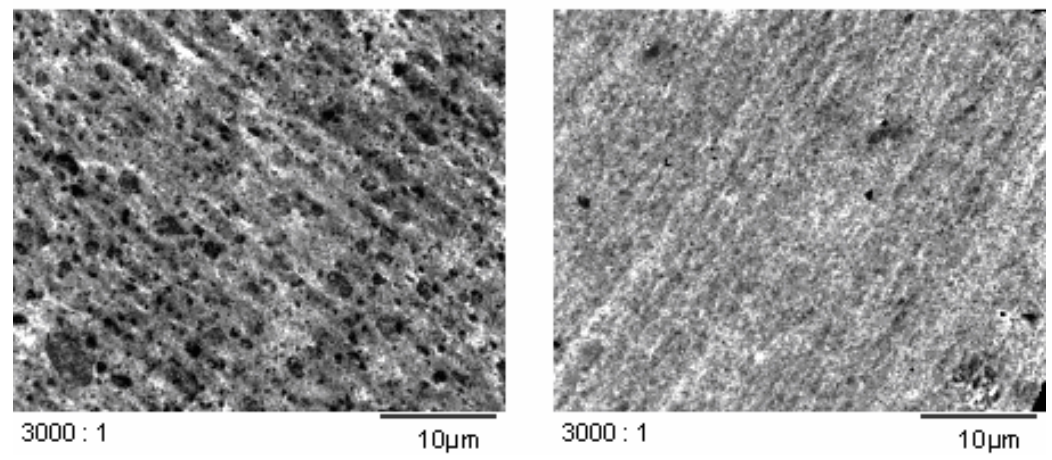

Figure 2.29: TEM images of good (right) and bad (left) micro-dispersion

This procedure contains certain limitations [106-107]. The sample preparation is relatively time consuming and cost-intensive. Additionally, a high number of images has to be analysed to get a statistically trustworthy result but furthermore, it highly depends on the operators' perspective of view. Another critical point is that the obtained three-dimensional information is transferred to a two-dimensional picture as shown in Fig. 2.30.

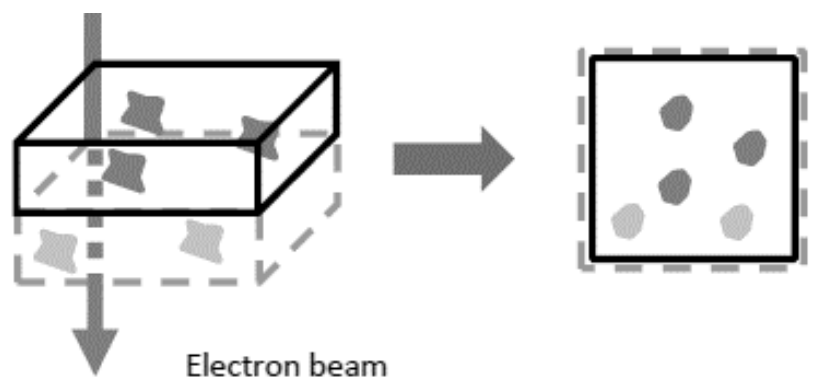

Figure 2.30: Transformation of the three-dimensional information to a two-dimensional picture for a TEM evaluation [108]

Additionally, the result strongly depends on the sample thickness. It is e.g. not possible to differ between two clusters positioned one after the other. It seems to be that for higher filler loadings, reliable and reproducible results cannot be obtained [108]. However, Julve et al. [109] published a paper including a method to determine the dispersion quality of silica in a highly loaded vulcanized compound. 


\section{Scanning Electron Microscopy (SEM)}

The scanning electron microscopy (SEM) technique is a further development of the TEM method. An electron beam scans the surface of a rubber sample (Fig. 2.31). For this, usually a 10 times lower voltage than that for TEM is used that the electrons do not pass through the sample and damage it. Some electrons are directly reflected, others penetrate into the surface and induce additional electrons to emit. Those secondary electrons are detected as well as the reflected electron beam and their signals are assembled into a two-dimensional image containing information of the rubber surface. This technique can be applied only for conductive surfaces. Non-conductive materials can be coated by a several $\mathrm{nm}$ thin layer of metal, e.g. gold by sputtering or evaporating [110-111].

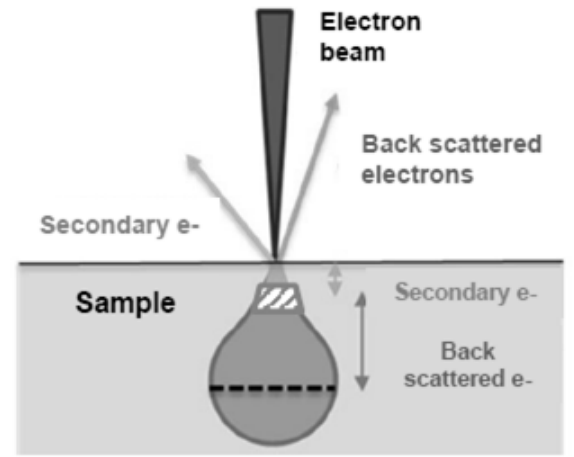

Figure 2.31: Functional principle of SEM [108]

\section{X-Ray Computed Microtomography (CT)}

The X-ray Computed Microtomography (CT) method is the first and up to now only technique to receive real three-dimensional information of filler macro-dispersion and distribution throughout a rubber matrix. Originally developed for medical purposes, it is nowadays used for a wide range of non-destructive investigations of materials. In principle, an X-ray cone beam passes a sample and is partly absorbed by different elements of the rubber compound. The remaining beam energy is detected (Fig. 2.32). Due to different densities and absorption coefficients of the elements, a two-dimensional map is generated [112].

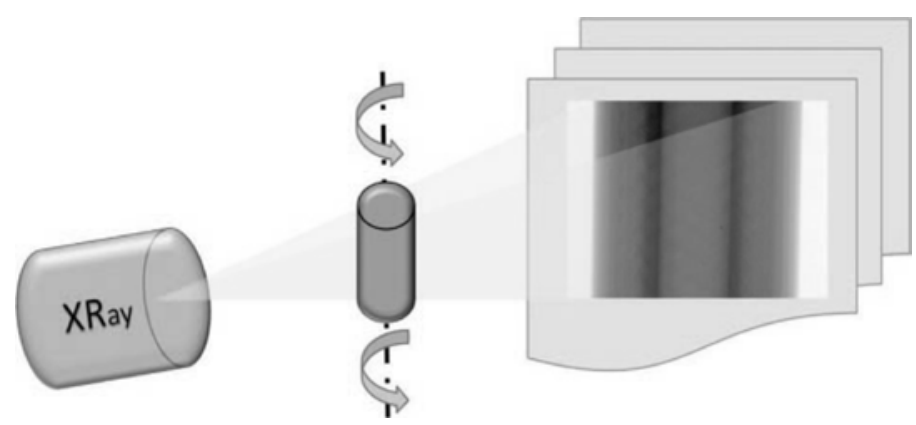

Figure 2.32: Schematic view of a CT [112]

To receive a three-dimensional information the sample has to be step by step completely rotated $\left(360^{\circ}\right)$. For each angle of rotation a new two dimensional image is generated. With the use of the Feldkamp algorithm [113] the three-dimensional picture is reconstructed (Fig. 2.33) and the particle size distribution can be evaluated. 


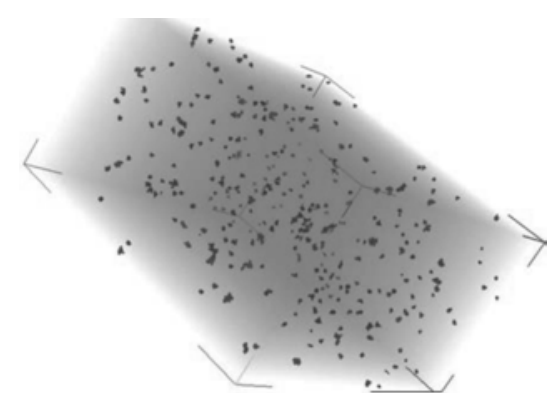

Figure 2.33: Schematic view of a 3D-CT image [114]

The advantages of this method are described in the following $[112,114]$ : No special sample preparation has to be performed. The size of the investigated area can be much larger in comparison to other dispersion measurement methods mentioned previously and, due to this, the gathered information is more representative. Therefore, even undispersed particles on a larger scale (up to $250 \mu \mathrm{m}$ ) can be evaluated. An additional use of X-ray spectrometry allows the characterization of the elementary composition and distinguishes between filler (e.g. silica), undispersed $\mathrm{ZnO}$ and trapped air. However, up to now, this method is limited to macro-dispersion at higher scale $(>6 \mu \mathrm{m})[112]$.

\subsubsection{Influences on dispersion quality}

The dispersion quality of fillers inside a rubber matrix can mainly be influenced by four factors: The mixing equipment, the mixing process, the compound formulation and the filler properties. This is described in the following.

\section{The mixing equipment / type of kneader}

The dispersion process of fillers occurs during the mixing process. Compounding usually is a batch wise process that is either realized on a two roll mill or inside an internal mixer. However, the exclusively use of a two roll mill is not state of the art anymore. Mixing on a two roll mill has certain disadvantages. It takes a comparable long time, depends on the operator, has a limited throughput and the incorporation of a higher amount of fillers is challenging [1]. In case of silica the needed high temperature for the silanization reaction can hardly be achieved $[104,115]$. Therefore, mixing of rubber compounds is usually realized inside an internal mixer. In principle, it is distinguished between two types of rotors, tangential and intermeshing rotors (Fig. 2.34).

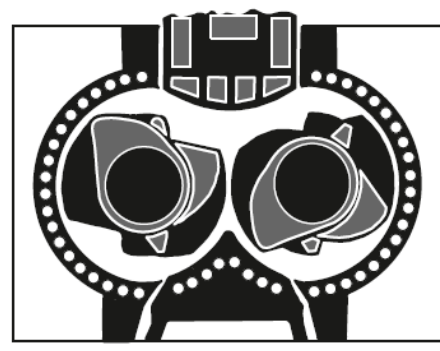

$\mathbf{N}$

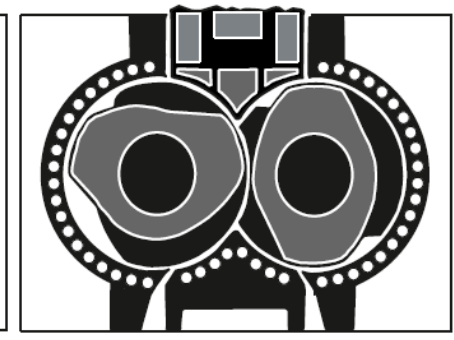

E

Figure 2.34: Typical rotor shapes of internal mixers - tangential (N) and intermeshing (E) [1]

Tangential rotors ("N") are characterized by a wide gap in between the rotors in such a way that their shape do not overlap during mixing. As a result, both rotors can run at different speeds. The material is forced into the gap and the incorporation of e.g. fillers is improved. This step is named the "fast feeding" step. The fill factors are usually higher than for intermeshing mixers and the mixing time is reduced. Therefore, tangential rotor geometries are preferred when a high machine efficiency is required [116]. 
Intermeshing rotors ("E") overlap during mixing and as a consequence they have to run at the same rotation speed. Due to the higher surface area of the rotors the temperature during mixing can be controlled more effectively which means that the mixer provides a higher cooling efficiency. This is especially for silica/silane filled compounds a big advantage. The narrow gap in between the rotors leads to higher shear forces and thus to an improved dispersive mixing. Intermeshing rotor geometries are preferred when a high mixing efficiency is required [116].

Beside the type of rotor, the rotor geometry itself has an impact on the final compound properties. The shape, number, length and setting of the wings can be varied which affect the material flow, occurring shear forces and therefore the dispersion process and efficiency [104].

\section{The mixing process}

During the mixing process a variety of parameters can be varied which have an impact on the dispersion process and therefore on the final dispersion quality. The most important ones are the mixing time and rotor speed, the mixing temperature, the fill factor and ram pressure and the order of adding ingredients. Additionally, the silanization reaction plays an important role.

\section{Mixing time and rotor speed}

The mixing time and rotor speed have both a major impact on the macro-dispersion quality. Schuster [104] showed that an increase of both parameters in a carbon black filled compound firstly leads to an improved macro-dispersion ending in a plateau (Fig. 2.35), where the dispersion quality cannot be improved any further within the given mixing conditions. The reason for this is that with increasing mixing time and rotor speed the temperature of the compounds increases as well and hence the viscosity decreases. Therefore, less energy can be transmitted to break down bigger agglomerates into smaller aggregates. Additional investigations of Geisler [117] pointed out that an increase of one minute in mixing time at early stages of the mixing phase improves the filler dispersion quality much more than an increase of one minute at a later stage where the dispersion quality hardly changes at all.

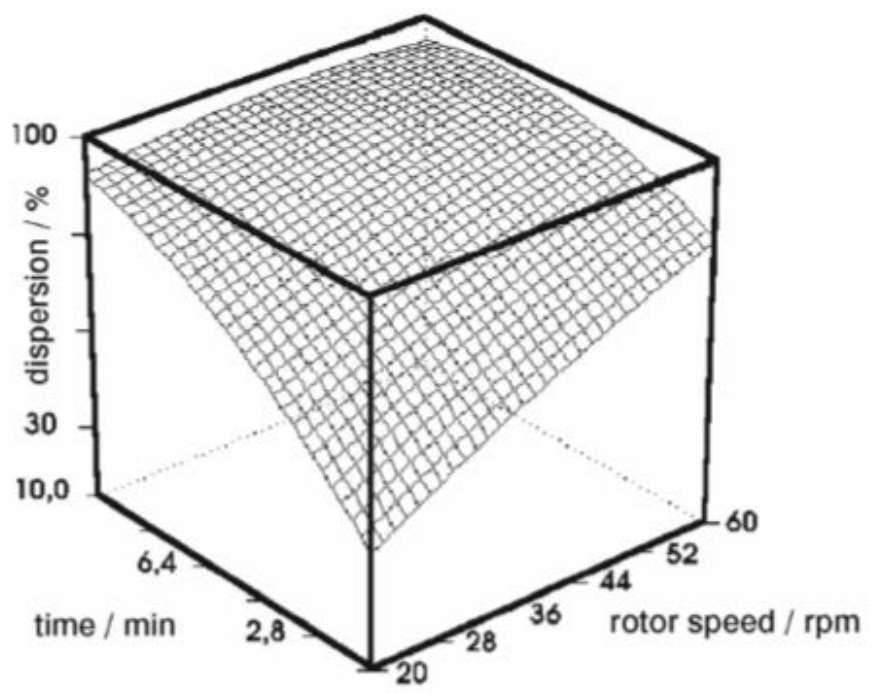

Figure 2.35: Influence of mixing time and rotor speed on carbon black macro-dispersion (light-optical dispersion measurement) [104]

\section{Mixing temperature}

A change in mixing temperature does affect the dispersion process in two ways. On the one hand, a higher temperature increases the polymer chain mobility and therefore its ability to penetrate faster into free voids of the filler structure [118]. The incorporation is improved. On the other hand, a higher mixing temperature leads to a lower compound viscosity. This results in lower shear forces and therefore lower dissipated energy which reduces the size of filler clusters less effectively [104]. Richmond [118] investigated the influence of the dump temper- 
ature on the macro-dispersion quality in a carbon black filled passenger car tire tread compound. With an increase of the dump temperature from $120^{\circ} \mathrm{C}$ up to $200{ }^{\circ} \mathrm{C}$, the macro dispersion quality is improved with an almost linear correlation. When using a silica / silane filler system, the mixing temperature additionally effects the silanization reaction and therefore influences the dispersion quality.

\section{Silanization reaction}

During the mixing of a silica / silane filler system it is not only necessary to achieve a good dispersion of silica itself but also to obtain an effective coupling of the silane to the silica surface. On the one hand, the better the dispersion of the silica, the higher the available surface area to react with the coupling agent. On the other hand, an optimum silanization reaction decreases the strong tendency of silica to re-agglomerate and leads to a better final in-rubber dispersion quality as well. As a consequence, the mixing has to be carried out in a certain processing window (Fig. 2.36) where the mixing temperature and rotor speed have to be adjusted in such a way that an optimum in silanization, dispersion quality and mixing time can be reached [7].

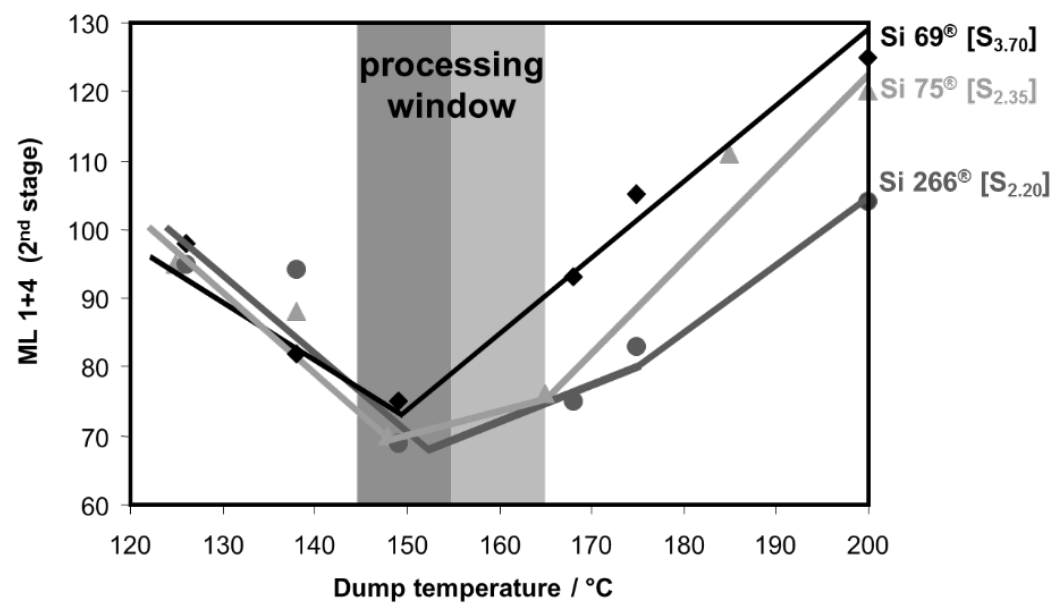

Figure 2.36: Processing window for an optimum silanization reaction for three different types of silane: bis-(triethoxysilyl-propyl)tetrasulfide (Si 69 ${ }^{\circledR}$ ) and bis-(triethoxysilyl-propyl)disulfide

(Si $266^{\circledR}$ and Si $75^{\circledR}$ ) with slightly different sulfur length [119]

Mixing below a certain temperature increases the mixing time due to a slower reaction between the silica and the silane. However, the higher viscosity at lower mixing temperature generates higher shear forces to overcome strong filler-filler interactions. When the mixing temperature exceeds the recommended range, there is a risk of pre-scorch which implies an unwanted early coupling of the silane towards the polymer, leading to processing difficulties [7].

\section{Fill factor and ram pressure}

The effect of fill factor and ram pressure on the dispersion quality is shown in Fig. 2.37. The fill factor is defined as the ratio of the compound volume and the free volume of the mixer. At low fill factors the distributive mixing is more pronounced whereas at higher degrees of filling the dispersive mixing prevails. Overloading the mixing chamber leads to an increase in mixing time and power consumption. The temperature increases, therefore the viscosity decreases which affects the dispersion process in a negative way. Additionally, the free volume or voids inside the mixer are reduced which are necessary for a sufficient distributive mixing and homogeneity of the compound $[116,120]$. 


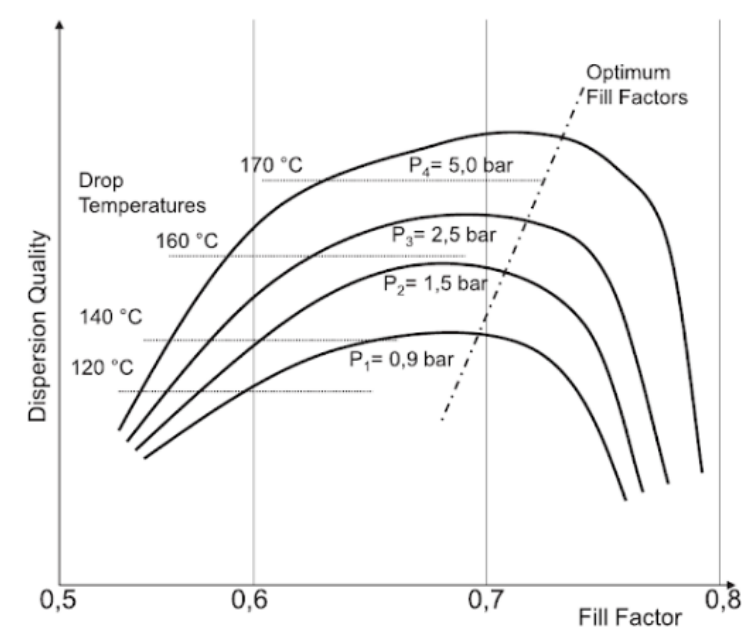

Figure 2.37: Influence of the fill factor and ram pressure on the dispersion quality [121]

A higher ram pressure at a fixed fill factor leads to a higher drop temperature and better dispersion quality. The incorporation is enhanced and therefore the dispersion process starts at an earlier stage. The ram position can be seen as an indicator for the incorporation process, the faster the ram settles to its final position the faster the incorporation of the filler occurs. And the earlier the filler is incorporated the earlier the dispersion process can start $[81,104,116]$.

\section{The order of adding ingredients}

When mixing rubber compounds, the order and timing of adding all ingredients affect the effective dispersive mixing to a great extent. One main factor is the change of viscosity of the compound e.g. by adding processing oil. Typically for a silica-filled compound, the mixing is separated in three stages where the first two mixing stages are usually carried out in an internal mixer. In the first stage, polymer is added, followed by the fillers and additional ingredients like processing oil and protecting agents. During this process the compound temperature rises and the viscosity drops which reduces the effective dispersive mixing. Therefore, the batch is stored after the first mixing stage for a certain amount of time to cool down. During the second mixing stage the cold compound is re-mixed and the higher viscosity results in more effective dispersive mixing again. The last stage is either be mixed inside an internal mixer or on a two-roll mill. The vulcanisation system is added and therefore the mixing is done at a low temperature to avoid pre-scorch [1].

An alternative proceeding is the upside-down mixing where all ingredients besides the polymer and vulcanisation system are added to the mixer at once followed by the polymer. Especially when using a high amount of oil, there is a certain risk of lubricating the surfaces of the rotors and chamber wall resulting in a less effective mixing. When adding the oil together with the filler first and after that the polymer, it can be absorbed onto the surface of the filler. As a result, the amount of free oil is sufficiently reduced and a more effective mixing can be achieved $[1,104]$.

Especially when mixing compounds including a silica-silane system different side reactions can occur. Reuvekamp [122] investigated the effect of adding zinc oxide and silane at different mixing times showed the impact on viscosity and pre-scorch.

\section{The compound formulation}

Different ingredients added to the compound formulation can influence the final dispersion quality as well. The most important ones are the polymer type and blend system, the type of processing oil, silane and silica itself.

In general, the interaction between the polymer and the filler is the better, the easier it is to reduce bigger clusters into smaller sizes. Polymer chains can either be physically absorbed or 
chemically linked to the filler surface. The compatibility of both materials strongly depends on their polarity, solubility parameter and chemical nature. In case of natural rubber e.g. it was shown that a higher molecular weight provided a significant improved macro-dispersion quality as long as the CB loading is kept below $80 \mathrm{phr}$ [104]. An experiment with one type of CB mixed into three polymers with different contents of styrene and vinyl groups demonstrates a significant influence on the macro-dispersion quality as can be seen in Fig. 2.38.

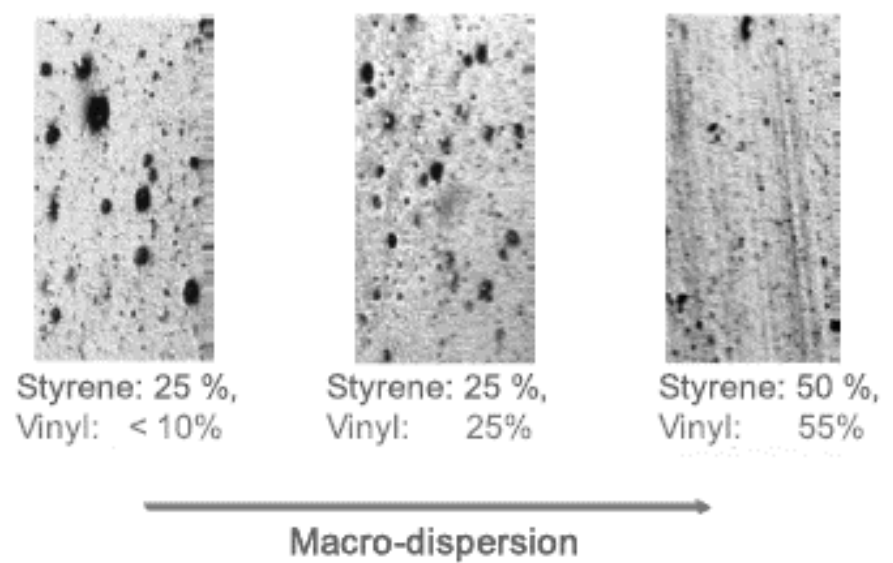

Figure 2.38: Influence of styrene and vinyl content on the macro-dispersion quality of $C B$ [104]

Moreover, the affinities of fillers to different types of polymers within a blend system varies during the mixing process [104]. Therefore, the distribution inside the different rubber phases differs as well and influences the dispersion process and hence the final dispersion quality [123]. Highly filled rubber compounds in general possess a relatively high viscosity resulting in a difficult processing. Therefore, it is recommended to add a higher quantity of processing oil to the compound. These mineral oils compete with the rubber being adsorbed on the fillers' surface. Due to their lower molecular weight and higher mobility, they tend to be adsorbed more easily, the adsorption of polymer chains is hindered. This results in lower rubber-filler interactions during the mixing and as a consequence the dispersion process is more difficult [124]. Fig. 2.39 depicts the influence of 10,25 and $40 \mathrm{phr}$ of oil on the macro-dispersion quality of $C B$ inside an SBR compound.

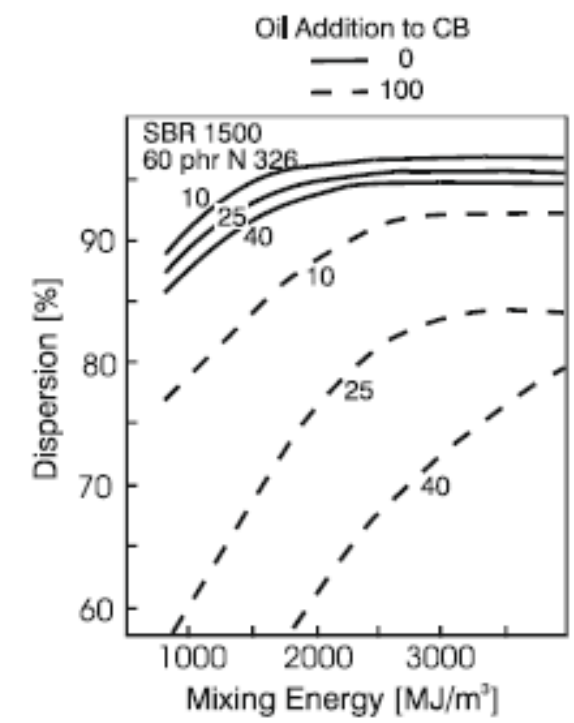

Figure 2.39: Influence of 10, 25 and 40 phr of oil and moment of its addition to the macro-dispersion quality of CB as a function of mixing energy [104] 
When the oil is added together with the filler (- - 100) the dispersion quality is reduced with increasing amount of oil. However, if CB is added to the rubber first, the polymer chains can be adsorbed first and the oil is only added after the filler incorporation $(-0)$, hardly any differences in the dispersion quality can be seen.

Beside the processing oil, silane is claimed to be a dispersion aid for silica as well. As described beforehands the silanization reaction enhances the compatibility of the polymer with the surface of silica. Therefore, it can be assumed that higher polymer-filler interactions occur resulting in an improved effective dispersive mixing. Three different types of silica were investigated inside an identical rubber compound with and without the addition of silane [19]. The macrodispersion quality was measured as a function of mixing time. It was shown that for a conventional as well as a semi-highly dispersible silica type an improved dispersion quality could be achieved with the use of silane. In addition, these compounds reached the final dispersion quality at an earlier stage of mixing. A highly dispersible type of silica on the contrary did demonstrate a very good level of dispersion even without the use of silane. However, the use of silane also results in a decrease of the compound viscosity which can cause less shear forces for the dispersive mixing and hence lead to a worse dispersion quality.

\section{Filler properties}

The filler itself has a great impact on the in-rubber dispersion quality. As described in chapter 2.2 , various analytical silica parameters can be measured and their influence on the silanization reaction and dispersion process are depicted in Tab. 2.4.

Table 2.4: Typical analytical parameters and their influence on the dispersion process [91]

\begin{tabular}{|c|c|c|}
\hline Method & Standard & Influence on \\
\hline BET: specific surface area & ISO 9277 & filler-filler interac- \\
\hline CTAB: specific surface area & ISO $5794 / 1 G$ & tions / dispersion \\
\hline DOA: initial structure & ISO 19246 & $\begin{array}{l}\text { incorporation / dis- } \\
\text { nersion }\end{array}$ \\
\hline moisture content $\left(2 \mathrm{~h} / 105^{\circ} \mathrm{C}\right)$ & ISO $787 / 2$ & silica/silane reaction \\
\hline $\mathrm{pH}$ & ISO $787 / 9$ & silica/silane reaction \\
\hline sieve residue (Mocker) & ISO $787 / 18$ & $\begin{array}{l}\text { dispersion / rubber } \\
\text { surface defects } \\
\text { aranules stability for }\end{array}$ \\
\hline sieve analysis (Rotap) & ISO 5794/1F & $\begin{array}{l}\text { conveying / incorpo- } \\
\text { ration }\end{array}$ \\
\hline
\end{tabular}

Especially the structure and specific surface area of silica are said to directly influence the dispersion quality of a final rubber compound as shown in the following.

\section{Filler Structure and Surface Area}

The effect of different carbon black structures on the filler macro-dispersion quality is shown exemplarily in Fig. 2.40. Two types of carbon black with similar surface areas (Statistical Thickness Surface Area STSA [125]) and different structures (OAN [35], Compressed Oil Absorption Number COAN [126]) are compared in two compounds using the same formulation, mixing process and equipment. It is shown that after the incorporation of the fillers after the first three minutes, the higher structured N330 reaches a higher macro-dispersion quality within the same mixing time [89]. 


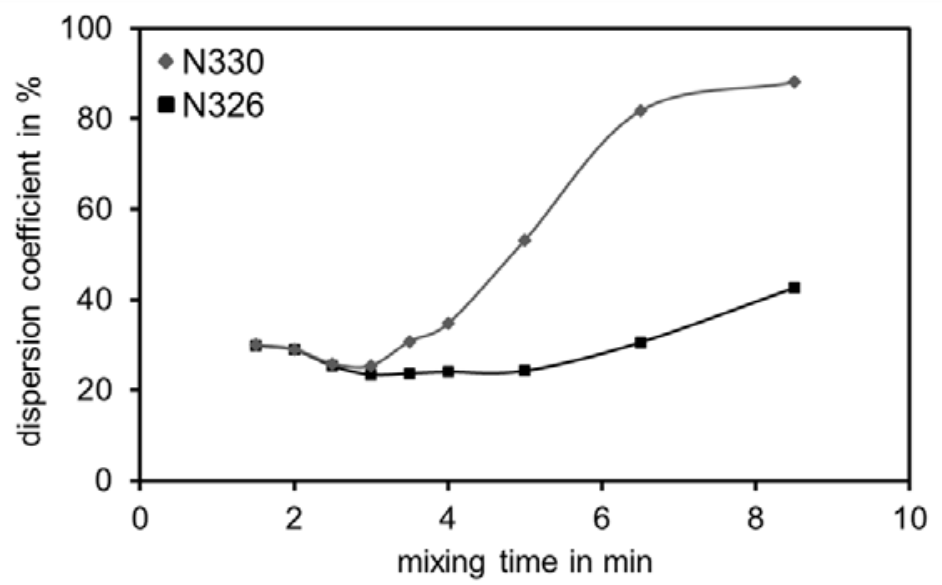

Figure 2.40: Influence of carbon black structure on dispersion quality [89]

Schuster [104] investigated the influence of different surface areas and structures of carbon black on the macro-dispersion quality measured by optical surface roughness (Fig. 2.41). It is shown that a higher structure generally leads to an improvement in the dispersion index. With an increase in the surface area of a low structured $C B$, the dispersion is improved whereas an increase of the surface area of a high structured CB leads to a decrease in dispersion quality.

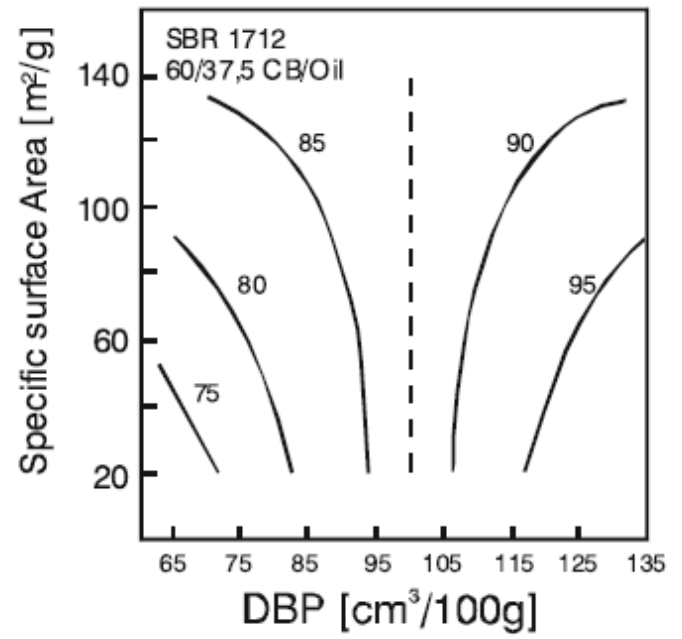

Figure 2.41: Influence of carbon black structure and specific surface area on dispersion quality [104]

Blume [127] investigated the influence of the BET surface area and the DBP value of silica on different rubber performances. Together with Uhrlandt [128] they demonstrated that a highly dispersible silica possesses a higher and less fragile structure than conventional silica grades.

\subsubsection{Influences of dispersion quality on rubber properties}

The dispersion quality of fillers inside a rubber compound strongly influences the dynamical and mechanical properties of the final product. A better dispersion quality in general leads to a higher reinforcement [104]. However, in some cases a perfectly dispersed and distributed filler is not desired [10]. In the following, the relevant influences of the filler dispersion quality on rubber properties are de-scribed.

\section{Rheological Properties}

A first effect caused by the dispersion quality of the filler in a rubber compound is a change in its rheological properties e.g. detected by the Mooney viscosity. In case of carbon black it is 
assumed that a better dispersion and distribution of the filler lead to a higher amount of polymer-filler interaction. Thereby the immobilized rubber is hindered in its mobility and partly increases the effective volume fraction of the filler. As a consequence, the viscosity increases which also influences the processibility of the compound [104].

\section{Fatigue Properties}

To investigate the influence of defects (respectively undispersed filler particles) on the fatigue to failure properties of rubber compounds, Abraham et al. [88] mixed glass beads of different diameters from $35 \mu \mathrm{m}$ up to $200 \mu \mathrm{m}$ into a EPDM compound. It was shown that the lifetime of a rubber product is decreased with increasing diameter of the glass beads and therefore it can be concluded that a better dispersion quality leads to improved fatigue properties.

\section{In-Rubber Properties}

Boonstra and Medalia [129] investigated various in-rubber properties as a function of the carbon black dispersion. By increasing the mixing time and therefore improving the dispersion quality, the tensile properties are enhanced, the permanent set, heat built-up and abrasion loss are reduced and the cut growth is improved. Based on this work, Payne [39] continued with the investigation of the dynamical properties of filler-loaded rubbers influenced by the dispersion quality. It was shown that the dynamical modulus, the viscous modulus and the phase angle at high strains are reduced with an improved dispersion quality at moderate oscillation amplitudes. Moreover, Cochet et al. [130] investigated the influence of the dispersion quality of precipitated silica on mechanical properties of different rubber compounds. It is stated that a better dispersion quality leads to an improvement of the tensile strength, elongation at break, abrasion and fatigue resistance.

\section{Rolling Resistance}

Nikiel et al. [131] showed that an improved carbon black dispersion quality achieved by increasing the mixing time leads to a reduction of the shear modulus G". This results in a lower hysteresis which is an indication for the desired improvement of the rolling resistance of a tire.

\section{Abrasion resistance}

Medalia [76] claimed that the macro-dispersion quality of carbon black strongly influences the abrasion resistance of passenger car tire treads. The same tendency was observed in real tire tests with silica / silane filled tire tread compounds [128] as shown in chapter 1 . A better macrodispersion, which means less undispersed filler-particles, results in a higher abrasion resistance and therefore a longer service life of tires.

\section{Crack propagation}

In case of off-the-road tire tread compounds, carbon black is usually partially replaced by silica to improve the tear and cut resistance [10]. A propagating crack can thereby be stopped by being trapped inside a silica network as shown in Fig. 2.42.

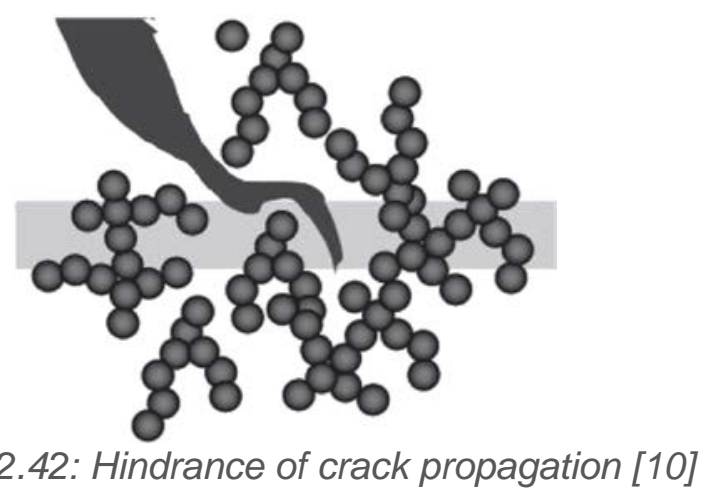

For this specific application, silica does not have to be perfectly dispersed and distributed and therefore a partly poor micro-dispersion quality is desired. 


\subsubsection{Prediction of dispersion quality}

The prediction of the dispersion quality respectively dispersibility of fillers by means of an analytical parameter was investigated by a few approaches as described in the following.

The PAYNE-Effect

The measurement of the PAYNE-effect is claimed to be an indirect measurement to predict the dispersion quality of fillers [132]. The higher the PAYNE-effect, the more pronounced a filler-filler network respectively the worse the distribution of filler particles. Therefore, this measurement corresponds to the micro-dispersion whereas the contribution of undispersed particles on a micro level is not determinable. However, this measurement has to be conducted with an already mixed compound and does not give any information about the analytical parameter of the filler and their effect on the dispersibility.

\section{WK-Coefficient}

One analytical method to describe the dispersibility of silica is the WK-coefficient [128]. The particle size distribution of a silica/water suspension is measured by means of a laser diffraction system after a defined sonification time. Usually, different types of silica show a bimodal particle size distribution in the size range of $40 \mathrm{~nm}$ to $500 \mu \mathrm{m}$. The main peak is situated around $10 \mu \mathrm{m}$ and corresponds to the original agglomerate structure. During sonification, silica is fragmented into aggregates possessing a peak around $400 \mathrm{~nm}$. The ratio of the height of the main peak to the second peak is referred to as the WK-coefficient. The smaller the coefficient, the easier it was to reduce the size of the silica respectively the better the dispersibility. However, newly developed types of silica do not necessarily demonstrate a bimodal distribution [133]. This method is therefore not applicable for all types of silica.

\section{Thermodynamic parameter}

Stöckelhuber et al. [79] defined the term dispersibility as a thermodynamic parameter of fillers. By investigating a few layers of filler particles fixed on an adhesive tape the dynamic contact angles could be assessed. With the calculated different surface energies and polarities in combination with measured surface energetic properties of the rubber, certain thermodynamic parameters were derived. In this way, the dispersibility, adhesion and flocculation behavior of fillers could be described.

\section{Laser Granulometry}

The laser granulometry [134] is a measurement system which monitors the particle size distribution of silica during an ultrasonic treatment. By recording certain characteristics of the distribution curve respectively their reduction over time, information about the speed of the particle size reduction can be obtained. The faster silica clusters get fragmented the easier they should be dispersed inside a rubber compound. This technique was developed for precipitated silica as therefore is been investigated in more detail in chapter 4.4.

\subsection{References}

[1] F. Röthemeyer; F. Sommer, Kautschuktechnologie, Carl Hanser Verlag, Munich (2013)

[2] J. Priestley, A familiar introduction to the theory and practice of perspective, Printed for

J. Johnson and J. Payne, London (1770)

[3] C. Goodyear, United States Patent Office, Improvement in india-rubber fabrics, US3633 (1844)

[4] J. B. Dunlop, The history of the pneumatic tyre, A. Thom \& co., Itd., (1925)

[5] P. J. Morris, Polymer Pioneers: A Popular History of the Science and Technology of Large Molecules, Chemical Heritage Foundation, (2005)

[6] R. Rauline, Compagnie Generale des Establissements Michelin et Cie, Rubber compound and tires based on such a compound, EP 0501227 B1 (1991)

[7] H.-D. Luginsland, A Review on the Chemistry and the Reinforcement of the SilicaSilane Filler System for Rubber Applications, Shaker Verlag, Cologne (2002)

[8] ASTM D1566:2015 - Standard Terminology Relating to Rubber

[9] DIN ISO 1629:2015-03 - Kautschuk und Latices - Nomenklatur 
[10] B. Rodgers, Rubber Compounding - Chemistry and Applications, CRC Press, Boca Raton - London - New York (2016)

[11] M. F. Sheridan, Rubber Handbook, 14, R.T. Vanderbilt Company, Inc., Norwalk, CT (2010)

[12] A. E. Boss, Adaptability - A tool for production development, Chem. Eng. News 27 (1949)

[13] R. K. Iler, The Chemistry of Silica, John Wiley \& Sons, New York - Chichester Brisbane - Toronto (1978)

[14] N. Hewitt, Compounding Precipitated Silica in Elastomers, William Andrew Publishing, Norwich, NY 13815 (2007)

[15] A. Wehmeier, Precipitated Silica, Evonik Industries "Rubber Seminar", (2015)

[16] ISO 9277:2010 - Determination of the specific surface area of solids by gas adsorption - BET method

[17] ISO 5794:2010 - Rubber compounding ingredients - Silica, precipitated, hydrated - Part 1: Non-rubber tests

[18] A. Voet et al., Reinforcement of Elastomers by Silica, Rubber Chem. Technol. 50 (1977) 342-355

[19] A. Wehmeier, Incorporation and Dispersion of Silica, presented at: 111. DKG West Bezirksgruppentagung, Bad-Neuenahr-Ahrweiler, Germany (2018)

[20] ISO 19246:2016 - Rubber Compounding Ingredients - Silica - Oil absorption of precipitated silica

[21] DIN 66133:1993-06 - Determination of pore volume distribution and specific surface area of solids by mercury intrusion

[22] A. Blume, Operando infrared study of the reaction of triethoxypropylsilane with silica, Kautsch. Gummi Kunstst. 8 (2008) 359-362

[23] O. Stenzel et al., EP1764344 (A2) - Precipitated silica having a specific pore size distribution, (2007)

[24] R. Mushack et al., White fillers in elastomers, Eur. Rubber J. 178 (1996) 24

[25] W. B. Wiegand, Reinforcement of rubbers, Ind. Rubber J. 60 (1920) 397-453

[26] W. B. Wiegand, Tendencies in rubber compounding, Trans. Inst. Rubber Ind. 1 (1925) 141

[27] A. Einstein, Ann. der Physik 19 (1906) 289-306

[28] A. Einstein, Ann. der Physik 34 (1911) 591-592

[29] E. Guth; O. Gold, On the hydrodynamical theory of the viscosity of suspensions, Phys. Rev. 53 (1938) 322

[30] H. M. Smallwood, Limiting Law of the Reinforcement of Rubber, J. Appl. Phys. 15 (1944) 758-766

[31] E. Guth, Theory of filler reinforcement, J. Appl. Phys. 16 (1945) 20-25

[32] S. Wolff; J.-B. Donnet, Characterization of fillers in vulcanizates according to the Einstein-Guth-Gold equation, Rubber Chem. Technol. 63 (1990) 32-45

[33] A. I. Medalia, Effective degree of immobilization of rubber occluded within carbon black aggregates, Rubber Chem. Technol. 45 (1972) 1171-1194

[34] A. I. Medalia, Elastic modulus of vulcanizates as related to carbon black structure, Rubber Chem. Technol. 46 (1973) 877-896

[35] ASTM D 2414:2016 - Standard Test Method for Carbon Black - Oil Absorption Number (OAN)

[36] P. P. A. Smit, Glass Transition in Carbon Black Reinforced Rubber, Rubber Chem. Technol. 41 (1968) 1194-1202

[37] I. Pliskin; N. Tokita, Bound rubber in elastomers: Analysis of elastomer-filler interaction and its effect on viscosity and modulus of composite systems, J. Appl. Sci. 16 (1972) 473-492

[38] S. Mihara, "Reactive processing of silica-reinforced tire rubber", 2009, ETE, University of Twente, Enschede

[39] A. R. Payne, Effect of dispersion on dynamic properties of filler-loaded rubbers, Rubber Chem. Technol. 39 (1966) 365-374 
[40] A. R. Payne; R. E. Whittaker, Low strain dynamic properties of filled rubbers, Rubber Chem. Technol. 44 (1971) 440-478

[41] J.-B. Donnet, Black and White Fillers and Tire Compound, Rubber Chem. Technol. 71 (1998) 323-341

[42] S. Wolff; M.-J. Wang, Filler-Elastomer Interactions. Part IV. The Effect of the Surface Energies of Fillers on Elastomer Reinforcement, Rubber Chem. Technol. 65 (1992) 329

[43] M.-J. Wang et al., Filler-Elastomer Interactions. Part VIII. The Role of the Distance between Filler Aggregates in the Dynamic Properties of Filled Vulcanizates, Rubber Chem. Technol. 66 (1993) 178-195

[44] M.-J. Wang, Effect of Polymer-Filler and Filler-Filler Interaction on Dynamic Properties of Filled Vulcanizates, Rubber Chem. Technol. 71 (1998) 520-589

[45] H.-D. Luginsland et al., Influence of Different Silanes on the Reinforcement of SilicaFilled Rubber Compounds, Rubber Chem. Technol. 75 (2002) 563-579

[46] M.-J. Wang; S. Wolff, Filler-Elastomer Interactions. Part V. Investigation of the Surface Energies of Silane-Modified Silicas, Rubber Chem. Technol. 65 (1992) 715-735

[47] S. Wolff, Reinforcement and Vulcanization Effects of Silane Si 69 Silica-Filled Compounds, Kautsch. Gummi Kunstst. 34 (1981) 280-284

[48] U. Görl et al., Investigation into the silica/silane reaction system, Rubber Chem. Technol. 70 (1997) 608-623

[49] A. Hunsche, Investigations Concerning the Reaction Silica/Organosilane and Organosilane/Polymer - Part 2, Kautsch. Gummi Kunstst. 51 (1998) 525-533

[50] A. Hunsche, Investigations Concerning the Reaction Silica/Organosilane and Organosilane/Polymer - Part 2, Kautsch. Gummi Kunstst. 50 (1997) 881-889

[51] A. Blume, Infrared Study of the Silica / Silane Reaction, presented at: $11^{\text {th }}$ Fall Rubber Colloquium, Hanover, Germany (2014)

[52] P. J. Nieuwenhuizen et al., Thiuram- and dithiocarbamate-accelerated sulfur vulcanization from the chemist's perspective: methods, materials and mechanisms reviewed, Rubber Chem. Technol. 70 (1997) 368-429

[53] A. Hasse et al., Influence of the amount of TESPT and sulfur on the reinforcement of silica-filled rubber compounds, Kautsch. Gummi Kunstst. 55 (2002) 236-243

[54] M. Sato, "Reinforcing mechanisms of silica / sulfide-silane vs. mercapto-silane filled tire tread compounds", PhD: 2018, Elastomer Technology Engineering, University of Twente, Enschede, the Netherlands

[55] G. Kraus, J. Appl. Polym. Sci. 75 (1984) 329

[56] G. Heinrich; M. Klüppel, Structure and properties of reinforcing fractal filler networks in elastomers, Rubber Chem. Technol. 70 (1997) 243-255

[57] H. Lorenz et al., Microstructure-based modelling and FE implementation of fillerinduced stress softening and hysteresis of reinforced rubbers, ZAMM 8 (2012) 608-631

[58] D. F. Twiss, J. Soc. Chem. Ind. 44 (1925) 1067

[59] G. Kraus, Interaction of elastomers and reinforcing fillers, Rubber Chem. Technol. 38 (1965) 1070-1114

[60] J. B. Donnet; A. Voet, Carbon Black: Physics, Chemistry, and Elastomer Reinforcement, M. Dekker, (1976)

[61] M.-J. Wang; S. Wolff, Surface Energy of Carbon Black, Carbon Black, Chapter 6 (Ed. J.-B. Donnet), Marcel Dekker Inc., New York (1993)

[62] J. M. Funt, Dynamic testing and reinforecement of rubber, Rubber Chem. Technol. 61 (1987) 842-865

[63] P. G. Maier; D. Göritz, Molecular Interpretation of the Payne Effect, Kautsch. Gummi Kunstst. 49 (1996) 18-21

[64] J. Berriot et. al., Evidence for the Shift of the Glass Transition near the Particles in Silica-Filled Elastomers, Macromolecules 35 (2002) 9756-9762

[65] C. Wrana; V. Härtel, Dynamic Mechanical Analysis of Filled Elastomers, Kautsch. Gummi Kunstst. 12 (2008) 647-655

[66] A. Blume, Lecture: Reinforcement, presented at: Elastomer Science Technology Course, University of Twente (2018) 
[67] J. D. Ferry, Viscoelastic Properties of Polymers, 3rd ed., John Wiley \& Sons, New York (1980)

[68] W. K. Dierkes, "Economic mixing of silica-rubber compounds: interaction between the chemistry of the silica-silane reaction and the physics of mixing", 2005, ETE, University of Twente, Enschede

[69] D. E. Hall; J. C. Moreland, presented at the Am. Chem. Soc. Rubber Dev. conference, April 4-6, Dallas, Texas (2000)

[70] D. E. Hall; J. C. Moreland, Fundamentals of Rolling Resistance, Rubber Chem. Technol. 74 (2001) 525-539

[71] K. A. Grosch; A. Schallamach, Tire Friction on Wet Roads, Rubber Chem. Technol. 49 (1976) 862-908

[72] M. L. Williams; R. L. Landel; J. D. Ferry, The Temperature Dependence of Relaxation Mechanisms in Amorphous Polymers and Other Glass-forming Liquids, J. Am. Chem. Soc. 77 (1955) 3701-3707

[73] G. Heinrich, Dynamics of Carbon Black Filled Networks, Viscoelasticity and Wet Skid Behavior, Kautsch. Gummi Kunstst. 45 (1992) 173-180

[74] K. Bandzierz et al., Influence of Network Structure on Glass Transition Temperature of Elastomers, Materials 9 (2016)

[75] K. Bandzierz et al., Effect of Polymer Chain Modifications on Elastomer Properties, Rubber Chem. Technol. in-press, DOI: 10.5254/RCT.18.82685 (2017)

[76] A. I. Medalia, Microscopic estimation of carbon black dispersion, Rubber Age (1965) 82-93

[77] Der Duden, Band 5, Das Fremdwörterbuch, Duden Verlag Bibliographisches Institut, Berlin (2015)

[78] ASTM D3053:2015 - Standard Terminology Relating to Carbon Black

[79] K. W. Stöckelhuber et al., Contribution of physico-chemical properties of interfaces on dispersibility, adhesion and flocculation of filler particles in rubber, Polymer 51 (2010) 1954-1963

[80] D. F. Bagster; D. Tomi, The stresses within a sphere in simple flow fields, Chem. Eng. Sci. 29 (1974) 1773-1783

[81] H. Palmgren, Processing Conditions in the Batch-Operated Internal Mixer, Rubber Chem. Technol. 48 (1975) 462-494

[82] J. M. Funt, Principles of mixing and measurement of dispersion, Rubber World (1986) 21-32

[83] N. Nakajima; E. R. Harrell, Contributions of Elastomer Behavior to Mechanisms of Carbon Black Dispersion, Rubber Chem. Technol. 57 (1984) 153-167

[84] V. Collin et al., New Insights in Dispersion Mechanisms of Carbon Black in a Polymer Matrix Under Shear by Rheo-Optics, J. Appl. Polym. Sci. (2013) 2121-2131

[85] E. Peuvrel-Disdier et al., Dispersion mechanisms of fillers in an elastomer matrix: What did we learn?, presented at: 111th Anniversary Meeting of DKG West Regional Group, Bad Neuenahr-Ahrweiler, Germany (2018)

[86] P. Grosseau et al., Internal structure and fragmentation kinetics of silica granules presented at: Powders and Grains, Sydney (2013)

[87] S. V. Kao; S. G. Mason, Dispersion of particles by shear, Nature International Journal of Science 253 (1975) 619-621

[88] F. Abraham et al., Untersuchung und Simulation des Einflusses von Fehlstellen auf das Risswachstum und die Ermüdung von Elastomeren, Kautsch. Gummi Kunstst. 58 (2005) 595-599

[89] A. Wehmeier, "Entwicklung eines Verfahrens zur Charakterisierung der Füllstoffdispersion in Gummimischungen mittels einer Oberflächentopographie", 1998, Fachhochschule Münster, Münster

[90] ASTM D 2663 - 14:2014 - Standard Test Method for Carbon Black - Dispersion in Rubber, Test Method C - Microroughness Measurement with Profilometer

[91] A. Wehmeier, Determination of the Macro-Dispersion of Advanced Fillers in Rubber Compounds, Evonik Industries Customer Seminar, Wesseling, Germany (2015) 
[92] T. Alshuth; R. H. Schuster, Raster-Kraft-Mikroskopie an Elastomerwerkstoffen - Teil 1. Charakterisierung von Rußmorphologie und Rußdispersion im „non-contact mode“, Kautsch. Gummi Kunstst. 47 (1994) 702-708

[93] L. L. Johnson, Atomic Force Microscopy (AFM) for Rubber, Rubber Chem. Technol. 81 (2008) 359-383

[94] N. Yerina; S. Magonov, Atomic Force Microscopy in Analysis of Rubber Materials, Rubber Chem. Technol. 76 (2003) 846-859

[95] N. Natchimuthu, AFM Studies on Silica Dispersion in EPDM Rubber, Rubber Chem. Technol. 83 (2010) 123-132

[96] ASTM D 2663:2014 - Standard Test Method for Carbon Black - Dispersion in Rubber, Test Method A - Visual Inspection

[97] L. Nikiel et al., Advances in Filler Dispersion Measurements, Rubber Age 89 (2016) 142-153

[98] ASTM D7723:2011 - Standard Test Method for Rubber Property - Macro Dispersion of Fillers in Compounds

[99] S. Otto et al., New Reference Value for the Description of Filler Dispersion with the Dispergrader 1000 NT, Kautsch. Gummi Kunstst. 58 (2005) 390-393

[100] R. H. Schuster et al., 2nd conference on Carbon Black, Mulhouse (France) (1992)

[101] M. Palm, "Charakterisierung der Mikrodispersion von Carbon Black in Gummi mit Hilfe eines konfokalen Lichtmikroskops", 2014, Technische Hochschule Nürnberg (2014)

[102] J. Kunkel, "Optimierung und Automatisierung des konfokalen Messverfahrens zur Bewertung der Makro-/Mikrodispersion rußgefüllter Elastomere und Auswirkungen der Dispersionsqualität auf die Abriebfestigkeit ausgewählter Vulkanisatproben", Masterarbeit: Technische Hochschule Nürnberg (2016),

[103] ASTM D 2663:2014 - Standard Test Method for Carbon Black - Dispersion in Rubber, Test Method B - Agglomerate Count

[104] A. Limper, Mixing of Rubber Compounds, Carl Hanser Verlag, Munich, Germany (2012)

[105] W. Hess, Characterization of Dispersions, Rubber Chem. Technol. 64 (1991) 386-449

[106] L. Conzatti et al., Microscopic imaging of rubber compounds; rubber technologist's handbook, Smithers Rapra Technology Limeted, Shrewsbury, Shropshire, U.K. (2009)

[107] P. E. F. Cudby; B.A. Gilbey, Scanning transmission imaging of elastomer blends using an unmodified conventional scanning electron microscope, Rubber Chem. Technol. 68 (1995) 342-350

[108] C. Fayolle, "Influence de la dispersion de la silice sur les propriétés viscoélastiques et mécaniques des élastomères renforcés", Doctoral Thesis: 2015, Université Claude Bernard - Lyon I

[109] D. Julve et al., Microdispersion of Silica in Tire Tread Compounds above the Percolation Threshold by TEM Image Measurements, Rubber Chem. Technol. 84 (2011) 74-87

[110] J. R. White; E. L. Thomas, Advances in SEM of Polymers, Rubber Chem. Technol. 57 (1984) 457-506

[111] M.C. Putman et al., High resolution reflected light microscopy to determine filler microdispersion, 172th technical fall meeting of the rubber division, ACS, Cleveland (USA) (2007)

[112] K. G. Jago, X-ray computed microtomography of rubber, Rubber Chem. Technol. 85 (2012) 387-407

[113] L. A. Feldkamp et al., Practical cone-beam algorithm, J. Opt. Soc. Am. A 1 (2010) 612619

[114] S. Robin; T. Alshuth, Hochauflösende 3D - Röntgen - Computertomographie (CT) Eine neue leistungsfähige Methode zur Charakterisierung von Elastomerwerkstoffen und Bauteilen, Kautsch. Gummi Kunstst. 63 (2010) 383-387

[115] H.-D. Luginsland et al., Optimal silica dispersion in mill, internal mixing, Rubber \& Plastic News August 9 (2004) 14-17

[116] W. M. Wiedmann; H. M. Schmid, Optimization of rubber mixing in internal mixers, Rubber Chem. Technol. 55 (1982) 363-381 
[117] H. Geisler, Dispersionskennfelder zur Überprüfung der Prozeßstabilität, 3. KautschukHerbst-Kolloquium, Hannover (1998)

[118] B. R. Richmond, Carbon Black Dispersion Measurement, Paper No. 68, American Chemical Society, Denver, Colorado (1993)

[119] H.-D. Luginsland; A. Hasse, Processing of Silica/Silane Filled Tread Compounds, Paper No. 34, American Chemical Society, Dallas, Texas (2000)

[120] E. S. Dizon, Processing in an Internal Mixer as Affected by Carbon Black Properties, Rubber Chem. Technol. 49 (1976) 12-27

[121] H. El Maanaoui et al., Computertomographische Untersuchung zur Partikelgrößenverteilung, DIK Deutsches Institut für Kautschuktechnologie (2018)

[122] L. A .E. M. Reuvekamp, "Reactive mixing of silica and rubber", PhD: 2003, Elastomer Technology Engineering, University of Twente, Enschede, The Netherlands

[123] J. Ziegler; R. H. Schuster, Dynamisch-mechanische Eigenschaften und Verteilung von Kieselsäure in NBR/BR-Verschnitten, Kautsch. Gummi Kunstst. 56 (2003) 159-165

[124] W. M. Hess et al., The influence of Carbon Black, mixing, and compounding variables on dispersion, Rubber Chem. Technol. 57 (1984) 959-1000

[125] ASTM D6556:2016 - Standard Test Method for Carbon Black - Total and External Surface Area by Nitrogen Adsorption

[126] ASTM D3493:2016 - Standard Test Method for Carbon Black - Oil Absorption Number of Compressed Sample (COAN)

[127] A. Blume, Analytical Properties of Silica a Key for Understanding Silica Reinforcement, Kautsch. Gummi Kunstst. 53 (2000) 338-345

[128] S. Uhrlandt and A. Blume, Kieselsäure für den Grünen Reifen - Prozesse, Produkte, Eigenschaften, Kautsch. Gummi Kunstst. 54 (2001) 520-527

[129] B. B. Boonstra and A. I. Medalia, Effect of carbon black dispersion on the mechanical properties of rubber vulcanizates, Rubber Chem. Technol. 36 (1963) 115-142

[130] Ph. Cochet et al., Dispersibility measurements of precipitated silicas - influence of dispersion on mechanical properties, Paper No. 162, American Chemical Society, Orlando, Florida, USA (1993)

[131] L. Nikiel et al., Filler Dispersion, Network Density, and Tire Rolling Resistance, Rubber Chem. Technol. 74 (2001) 249-259

[132] A. I. Medalia, Reinforcement of Elastomers, Encyclopedia of Materials: Science and Technology (2001)

[133] Internal Information, Evonik Resource Efficiency GmbH

[134] R. Valero; J. Hernandez, WO 03/016215 A1, Method of preparing silicas, silicas with specific pore-size and/or particle-size distribution and the use thereof, in particular for reinforcing polymers (2003) 


\section{Chapter 3 - Rubber Compounding and Testing}

The present chapter summarizes all formulations for the mixed compounds, the mixing processes and in-rubber tests to evaluate the dispersion quality of silica in different rubber formulations. Therefore, 25 different types of silica with a variety of standard analytical parameters and dosage forms were mixed into four compounds. The Green Tire compounds filled with 80 phr of silica were defined as the reference series. In addition, another Green Tire test series with 50 phr of silica were evaluated. Moreover, NR compounds filled with 55 phr of silica were investigated. All three test series included silane inside the formulation. Finally, Green Tire compounds filled with 80 phr of silica but without the use of silane were mixed.

The macro-dispersion qualities of all four series were evaluated by means of the Topography (mechanical) and Dispertester (optical) measurement systems. Furthermore, the PAYNE-effect was investigated, which is stated in the literature to be an indirect measurement for the dispersion quality as well.

In case of the reference series (GT80) special investigations were conducted on selected compounds. First of all, samples were evaluated by means of the Dispergrader, a measurement system comparable to the Dispertester. In addition, the dispersion quality was measured with a confocal light microscope and with an X-ray computed microtomography ( $\mu m-C T)$. Finally, the abrasion resistance of several compounds were evaluated by means of the laboratory abrasion tester (LAT100).

\subsection{Introduction}

As defined in chapter 2.4.1, the dispersibility of silica can in general only be assessed within the same formulation, using the same mixing equipment and mixing conditions. To achieve various different dispersion qualities and broadening the results, a wide variety of silica has therefore to be investigated. The dispersibility of silica is as well depending on the compound itself. Therefore, all selected fillers were tested within four different compound series. 25 different types of silica with a variety of analytical parameters were chosen to be investigated in the present work (Tab 3.1.). Three dosage forms can thereby be distinguished, granules (GR), powders (P) and micro-pearls (MP). The five standard analytical parameters of silica are specified and all silica grades are labelled as follows: The first number represents the specific surface measured by CTAB [1], the letter characterizes the dosage form and the last number describes the initial structure measured by DOA [2]. 
Table 3.1: 25 different types of silica and their five standard analytical parameters

\begin{tabular}{|c|c|c|c|c|c|}
\hline Silica & $\begin{array}{l}\text { CTAB [1] } \\
\text { in } \mathrm{m}^{2} / \mathrm{g} \\
\end{array}$ & $\begin{array}{c}\text { DOA [2] } \\
\text { in } \mathrm{ml} / 100 \mathrm{~g}\end{array}$ & $\begin{array}{l}\text { BET [3] } \\
\text { in } \mathrm{m}^{2} / \mathrm{g} \\
\end{array}$ & $\begin{array}{c}\text { moisture content } \\
\text { in } \%[4]\end{array}$ & $\begin{array}{c}\mathrm{pH}-\text { value } \\
{[5]}\end{array}$ \\
\hline 122GR195 & 122 & 195 & 120 & 3.8 & 7.1 \\
\hline 104GR175 & 104 & 175 & 107 & 5.8 & 6.7 \\
\hline 190GR197 & 190 & 197 & 208 & 5.7 & 6.7 \\
\hline 157GR186 & 157 & 186 & 152 & 6.2 & 6.7 \\
\hline 110GR205 & 110 & 205 & 120 & 5.9 & 6.8 \\
\hline 171GR196 & 171 & 196 & 199 & 4.8 & 6.6 \\
\hline 175GR203 & 175 & 203 & 198 & 5.2 & 6.8 \\
\hline 173GR196 & 173 & 196 & 190 & 4.6 & 6.9 \\
\hline 158GR209 & 158 & 209 & 153 & 6.0 & 7.0 \\
\hline 215GR210 & 215 & 210 & 215 & 4.7 & 6.6 \\
\hline 197GR199 & 197 & 199 & 213 & 8.1 & 6.6 \\
\hline 161GR193 & 161 & 193 & 163 & 6.6 & 6.6 \\
\hline 159GR204 & 159 & 204 & 163 & 6.1 & 6.5 \\
\hline 165GR181 & 165 & 181 & 185 & 5.2 & 6.1 \\
\hline 127P206 & 127 & 206 & 124 & 5.1 & 6.8 \\
\hline 173P223 & 173 & 223 & 203 & 4.9 & 6.6 \\
\hline 176P238 & 176 & 238 & 193 & 5.6 & 6.8 \\
\hline 195P228 & 195 & 228 & 225 & 5.8 & 6.5 \\
\hline 162P225 & 162 & 225 & 165 & 5.5 & 6.6 \\
\hline 155P213 & 155 & 213 & 162 & 6.5 & 6.6 \\
\hline 165P196 & 165 & 196 & 181 & 4.6 & 5.8 \\
\hline 157MP207 & 157 & 207 & 150 & 6.6 & 6.6 \\
\hline 173MP217 & 173 & 217 & 188 & 5.2 & 7.0 \\
\hline 176MP223 & 176 & 223 & 189 & 5.2 & 6.3 \\
\hline 114MP199 & 114 & 199 & 109 & 6.0 & 7.1 \\
\hline
\end{tabular}

It can be seen that roughly half of the types of silica chosen are granules due to the fact that this dosage form is most commonly used in the rubber industry. Powders are in general difficult to incorporate into the polymer and micro-pearls undergo a special production process. The five standard analytical parameters and their general impact on the in rubber performance are already described in chapter 2.2. These 25 types of silica were mixed in four different compound series and the macro-dispersion qualities were determined by means of the Topography [6-7] and the Dispertester [8] measurement. In addition, the PAYNE-effect [9] was evaluated. In case of the standard Green Tire formulation, additional tests were performed on selected compounds.

\subsection{Compound Series}

To achieve a variety of dispersion results all types of silica were mixed in the following four different compound series: The first one is a Green Tire formulation for passenger tire treads filled with $80 \mathrm{phr}$ of silica (GT80). This series represents the reference series for the present work. The second series is a similar Green Tire formulation but filled only with $50 \mathrm{phr}$ of silica (GT50). It is expected that the lower loading of filler leads to a lower viscosity, hence less energy input respectively shear forces and as a result a wider spreading of dispersion qualities in comparison to the GT80 series. The third series is a natural rubber formulation filled with $55 \mathrm{phr}$ of silica (NR55). Due to the naturally high molecular weight and hence high viscosity of NR [10] it is expected that even conventional silica are very well dispersed and hardly any differences in the dispersion qualities can be found. For these three different series silica was used in combination with silane. Finally, the Green Tire formulation filled with $80 \mathrm{phr}$ of 
silica was chosen again but without the use of silane (GT80 w/o). In this way, the influence of the bi-functional coupling agent on the dispersion process should be investigated.

All mixing processes were divided into three stages and mixed inside a $1.5 \mathrm{I}$ tangential mixer (Werner \& Pfleiderer, type 01.323). The fill factor, rotation speed of the rotors and chamber temperature were set to a fixed level without being adjusted during mixing. After each mixing stage the compounds were sheeted out on a two roll mill (Schwabenthan 3.0 - Servitec Maschinenservice $\mathrm{GmbH}$ ).

Series 1 - GT80: Green Tire formulation and mixing (80 phr of silica)

The Green Tire formulation filled with $80 \mathrm{phr}$ of silica was defined as the reference compound for the present work. Tab. 3.2 depicts the formulation used for this series.

Table 3.2: Green Tire formulation filled with 80 phr of silica (GT80) $1^{\text {st }}$ stage

\begin{tabular}{|c|c|c|}
\hline Material & type & phr \\
\hline Buna VSL 4526-2 & oil-extended S-SBR & 96.25 \\
\hline Buna CB 24 & Nd-BR; cis1,4 > $96 \%$ & 30.00 \\
\hline Silica & variable & 80.00 \\
\hline Si 266 & silane & adjusted to CTAB \\
\hline N330 & carbon black & 5.00 \\
\hline ZnO RS RAL 844 C & zinc oxide & 2.00 \\
\hline Edenor ST1 GS & stearic acid & 2.00 \\
\hline Vivatec 500 & TDAE* oil & 8.75 \\
\hline Vulkanox HS/LG & $\mathrm{TMQ}^{\star *}$ protector & 1.50 \\
\hline Vulkanox 4020/LG & $6 P P D^{\star \star \star}$ anti-aging & 2.00 \\
\hline Protektor G 3108 & Wax & 2.00 \\
\hline \multicolumn{3}{|c|}{$2^{\text {nd }}$ stage } \\
\hline \multicolumn{3}{|l|}{ batch $1^{\text {st }}$ stage } \\
\hline Rhenogran DPG-80 & $80 \%$ DPG ${ }^{\star \star \star \star}$ accelerator & 2.50 \\
\hline \multicolumn{3}{|c|}{$3^{\text {rd }}$ stage } \\
\hline \multicolumn{3}{|l|}{ batch $2^{\text {nd }}$ stage } \\
\hline Richon TBZTD OP & TBzTD***** accelerator & 0.20 \\
\hline Vulkacit CZ/EG-C & CBS ${ }^{\star \star \star \star \star \star}$ accelerator & 1.60 \\
\hline Sulfur $80 / 90$ & soluble sulfur & 2.00 \\
\hline
\end{tabular}

* TDAE: Treated Distillated Aromatic Extract

** TMQ: 2,2,4-TriMethyl-1,2-dihydroQuinoline

*** 6PPD: N-(1,3-dimethylbutyl)-N'-Phenyl-p-PhenyleneDiamine

**** DPG: N,N'-DiPhenylGuanidine

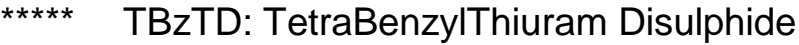

$\star \star \star \star \star * \quad$ CBS: N-Cyclohexyl-2-BenzothiazoleSulfenamide

The amount of silane (Si266) was adjusted to the specific surface area of silica (CTAB) to guarantee a sufficient hydrophobization of the polar surface of the filler. As a basis, the ratio of $5.8 \mathrm{phr}$ silane to $80 \mathrm{phr}$ of silica with a CTAB of $160 \mathrm{~m}^{2} / \mathrm{g}$ was chosen. The adjustment to other types of silica respectively CTAB surface areas was made by the rule of three. All other ingredients were kept constant so that differences in dispersion qualities should mainly be traced back to the analytical properties of the different types of silica itself. Tab. 3.3 shows the mixing process for the GT80 series. 
Table 3.3: Mixing process for the Green Tire compounds filled with 80 phr of silica

stage and time

\begin{tabular}{|c|c|}
\hline $\begin{array}{l}1^{\text {st }} \text { stage } \\
\text { min:sec }\end{array}$ & $\begin{array}{l}\text { fill factor } 0.73 ; 70 \mathrm{rpm} \text {; chamber temperature: } 70^{\circ} \mathrm{C} \\
\text { measured temperature: } 130-150^{\circ} \mathrm{C}\end{array}$ \\
\hline 00:00 - 00:15 & polymer \\
\hline 00:15 - 00:45 & 1/3 silica; $1 / 3$ silane \\
\hline $00: 45-01: 15$ & 1/3 silica; $1 / 3$ silane \\
\hline $01: 15-02: 15$ & $\begin{array}{l}\text { a) oil adsorbed on CB in a PE pouch } \\
\text { b) } 1 / 3 \text { silica; silane } \\
\text { c) protector }\end{array}$ \\
\hline $\begin{array}{l}02: 15-04: 15 \\
04: 15\end{array}$ & $\begin{array}{l}\mathrm{ZnO} \text {, stearic acid; Vulkanox HS; Vulkanox 4020; } \\
\text { dump and control temperature }\end{array}$ \\
\hline & $\begin{array}{l}45 \mathrm{~s} \text { on open mill ( } 4 \mathrm{~mm} \text { nip), sheet out } \\
\text { weigh compound for } 2^{\text {nd }} \text { step; storage for } 24 \mathrm{~h} / \mathrm{RT}\end{array}$ \\
\hline $\begin{array}{l}2^{\text {nd }} \text { stage } \\
\text { min:sec }\end{array}$ & $\begin{array}{c}\text { fill factor } 0.70 ; 70 \mathrm{rpm} \text {; chamber temperature: } 90^{\circ} \mathrm{C} \\
\text { measured temp.: } 130-150{ }^{\circ} \mathrm{C}\end{array}$ \\
\hline 00:00 - 01:00 & plasticize $1^{\text {st }}$ stage \\
\hline 01:00 - 03:00 & DPG; mix; \\
\hline 03:00 & dump and control temperature \\
\hline & $45 \mathrm{sec}$. on open mill ( $4 \mathrm{~mm}$ nip), sheet out \\
\hline $\begin{array}{l}3^{\text {rd }} \text { stage } \\
\text { min:sec }\end{array}$ & $\begin{array}{c}\text { fill factor } 0.68 ; 55 \mathrm{rpm} \text {, chamber temperature: } 50{ }^{\circ} \mathrm{C} \\
\text { measured temperature: }>110^{\circ} \mathrm{C}\end{array}$ \\
\hline 00:00 - 02:00 & batch stage 2; accelerators; sulfur \\
\hline $02: 00$ & $\begin{array}{l}\text { dump batch; process on open mill } 20 \text { sec. with } 3 \text { - } 4 \text { mm nip } \\
\text { cut } 3 \times \text { left, } 3 \times \text { right with } 3 \mathrm{~mm} \text { nip } \\
\text { roll up and pass through a } 3 \mathrm{~mm} \text { nip x } 3 \\
\text { sheet off; store for minimum } 12 \text { h before vulcanization }\end{array}$ \\
\hline
\end{tabular}

As mentioned beforehands, silica powder is more difficult to handle and to incorporate into the rubber during mixing in comparison to other dosage forms. Therefore, silica was added in three equal portions during the first stage. Fig. 3.1 depicts the fingerprint of the $1^{\text {st }}$ stage of mixing for one type of silica (155P213) including the ram position, the power and the temperature profile as a function of time. The rotor speed was kept constant throughout the whole mixing. 


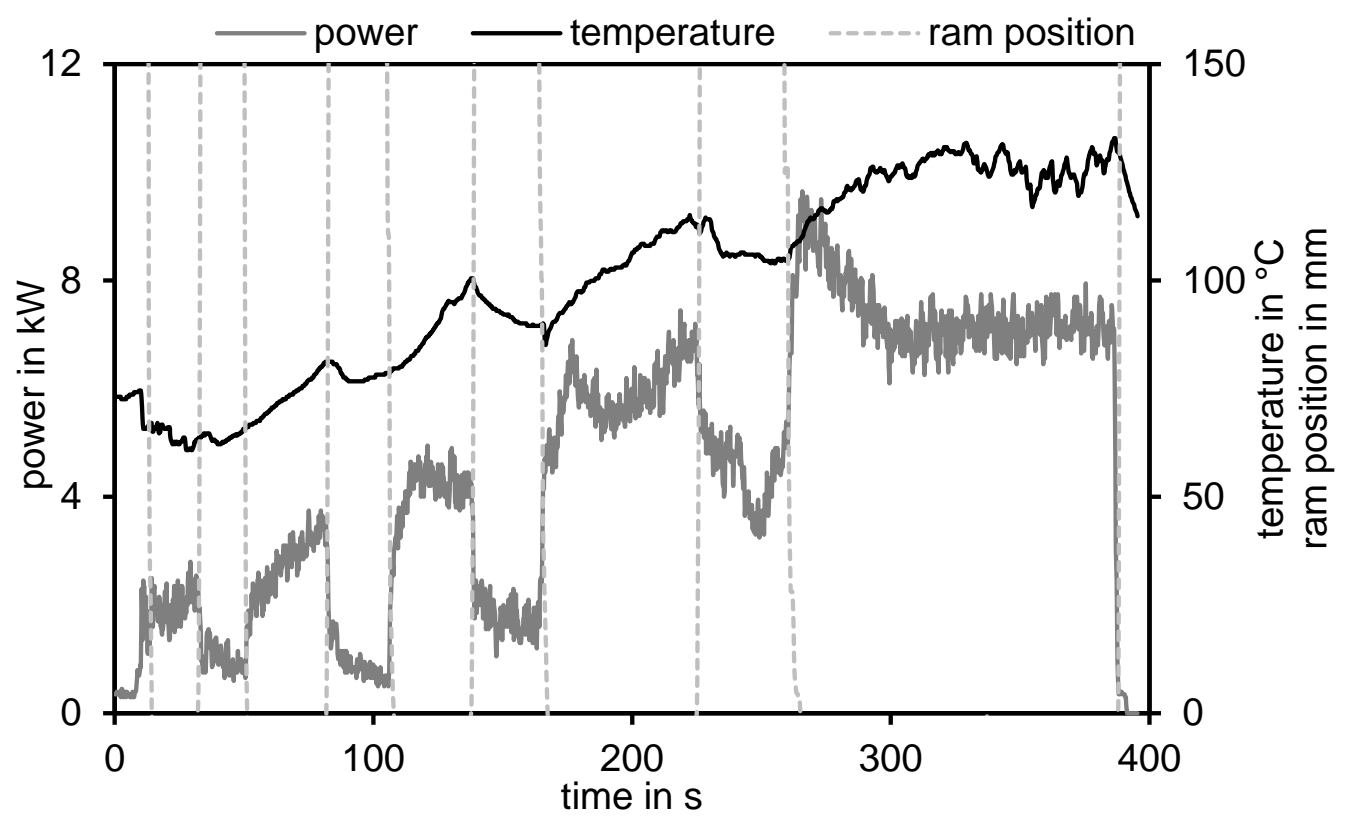

Figure 3.1: Fingerprint of the $1^{\text {st }}$ stage of the GT80 series

It can be seen that by adding the filler in three parts the ram was able to quickly settle to its final position and mixing could be continued. After the addition of the $3^{\text {rd }}$ portion of silica including the other ingredients the power increased strongly due to the fact that the optimum fill factor for the mixing chamber was reached. During the last part of the mixing, the temperature reached an optimum level of $+130^{\circ} \mathrm{C}$ where the silanization reaction takes place without a risk of pre-scorch (average power of $7 \mathrm{~kW}$ ). There was no need to adjust the rotor speed during mixing for any compound. The mixing of the stages 2 and 3 did not show any conspicuousness as well. 
Series 2 - GT50: Green Tire formulation and mixing (50 phr of silica)

The green tire formulation filled with $50 \mathrm{phr}$ of silica was chosen to be mixed in order to achieve a wider spreading of dispersion qualities in comparison to the standard Green Tire formulation (GT80). Tab. 3.4 depicts the formulation for series 2 - GT50:

Table 3.4: Green Tire formulation filled with 50 phr of silica (GT50) $1^{\text {st }}$ stage

\begin{tabular}{|c|c|c|}
\hline material & Type & phr \\
\hline Buna VSL 4526-2 & oil-extended S-SBR & 96.25 \\
\hline Buna CB 24 & Nd-BR; cis1,4 > $96 \%$ & 30.00 \\
\hline Silica & variable & 50.00 \\
\hline Si 266 & silane & adjusted to CTAB \\
\hline N330 & carbon black & 5.00 \\
\hline ZnO RS RAL 844 C & zinc oxide & 2.00 \\
\hline Edenor ST1 GS & stearic acid & 2.00 \\
\hline Vivatec 500 & TDAE oil & 8.75 \\
\hline Vulkanox HS/LG & TMQ protector & 1.50 \\
\hline Vulkanox 4020/LG & 6PPD anti-aging & 2.00 \\
\hline Protektor G 3108 & Wax & 2.00 \\
\hline \multicolumn{3}{|c|}{$2^{\text {nd }}$ stage } \\
\hline \multicolumn{3}{|l|}{ batch $1^{\text {st }}$ stage } \\
\hline Rhenogran DPG-80 & $80 \%$ DPG accelerator & 2.50 \\
\hline \multicolumn{3}{|c|}{$3^{\text {rd }}$ stage } \\
\hline \multicolumn{3}{|l|}{ batch $2^{\text {nd }}$ stage } \\
\hline Richon TBZTD OP & TBzTD accelerator & 0.20 \\
\hline Vulkacit CZ/EG-C & CBS accelerator & 1.60 \\
\hline Sulfur 80/90 & soluble sulfur & 2.00 \\
\hline
\end{tabular}

The formulation and mixing process were divided into three stages again and the amount of silane (Si266) was adjusted to the specific surface area of silica (CTAB) as well. As a basis, the ratio of $3.6 \mathrm{phr}$ silane to $50 \mathrm{phr}$ of silica with a CTAB of $160 \mathrm{~m}^{2} / \mathrm{g}$ was chosen. The adjustment to the other types of silica respectively surfaces areas was made by the rule of three. Tab. 3.5 shows the mixing process for the GT80 series. 
Table 3.5: Mixing process for the Green Tire compounds filled with 50 phr of silica

stage and time

\begin{tabular}{|c|c|}
\hline $\begin{array}{l}1^{\text {st }} \text { stage } \\
\text { min:sec }\end{array}$ & $\begin{array}{l}\text { fill factor } 0.73 ; 70 \mathrm{rpm} \text {; chamber temperature: } 70^{\circ} \mathrm{C} \\
\text { measured temperature: } 130-150^{\circ} \mathrm{C}\end{array}$ \\
\hline 00:00 - 00:15 & polymer \\
\hline 00:15 - 01:15 & 1/2 silica; silane \\
\hline 01:15 - 01:15 & cleaning step \\
\hline $01: 15-02: 15$ & $\begin{array}{l}\text { a) oil adsorbed on CB in a PE pouch } \\
\text { b) } 1 / 2 \text { silica } \\
\text { c) protector }\end{array}$ \\
\hline $\begin{array}{c}02: 15-03: 45 \\
03: 45\end{array}$ & $\begin{array}{l}\text { ZnO, stearic acid; Vulkanox HS; Vulkanox } 4020 \text {; } \\
\text { dump and control temperature } \\
45 \text { s on open mill ( } 4 \text { mm nip), sheet out } \\
\text { weigh compound for } 2 \text { nd step; storage for } 24 \text { h / RT }\end{array}$ \\
\hline $\begin{array}{l}2^{\text {nd }} \text { stage } \\
\text { min:sec }\end{array}$ & $\begin{array}{l}\text { fill factor } 0.70 ; 70 \mathrm{rpm} \text {; chamber temperature: } 90^{\circ} \mathrm{C} \\
\text { measured temperature: } 130-150{ }^{\circ} \mathrm{C}\end{array}$ \\
\hline 00:00 - 01:00 & plasticize $1^{\text {st }}$ stage \\
\hline 01:00 - 03:00 & DPG; mix; \\
\hline 03:00 & $\begin{array}{l}\text { dump and control temperature } \\
45 \mathrm{sec} \text {. on open mill ( } 4 \mathrm{~mm} \text { nip), sheet out } \\
\text { weigh compound for } 3 \mathrm{rd} \text { step; storage for } 4 \text { - } 24 \mathrm{~h} \text { / RT }\end{array}$ \\
\hline $\begin{array}{l}3^{\text {rd }} \text { stage } \\
\text { min:sec }\end{array}$ & $\begin{array}{l}\text { fill factor } 0.68 ; 55 \mathrm{rpm} \text {, chamber temperature: } 50{ }^{\circ} \mathrm{C} \\
\text { measured temperature: }>110^{\circ} \mathrm{C}\end{array}$ \\
\hline $\begin{array}{l}\text { 00:00 - 02:00 } \\
02: 00\end{array}$ & $\begin{array}{l}\text { batch stage } 2 \text {; accelerators; sulfur } \\
\text { dump batch; process on open mill } 20 \mathrm{sec} \text {. with } 3 \text { - } 4 \mathrm{~mm} \text { nip } \\
\text { cut } 3 \times \text { left, } 3 \times \text { right with } 3 \mathrm{~mm} \text { nip } \\
\text { roll up and pass through a } 3 \mathrm{~mm} \text { nip } \times 3 \\
\text { sheet off; store for minimum } 12 \text { h before vulcanization }\end{array}$ \\
\hline
\end{tabular}

With reduced filler loading to $50 \mathrm{phr}$, it was possible to add all types of silica in two equal portions during the first stage. After each stage the compounds were sheeted out by the help of a two roll mill and stored for the specified time. Fig. 3.2 depicts the fingerprint of the $1^{\text {st }}$ stage of mixing for one type of silica (162P225) including the ram position, the power and the temperature profile as a function of time. The rotor speed was kept constant throughout the whole mixing. 


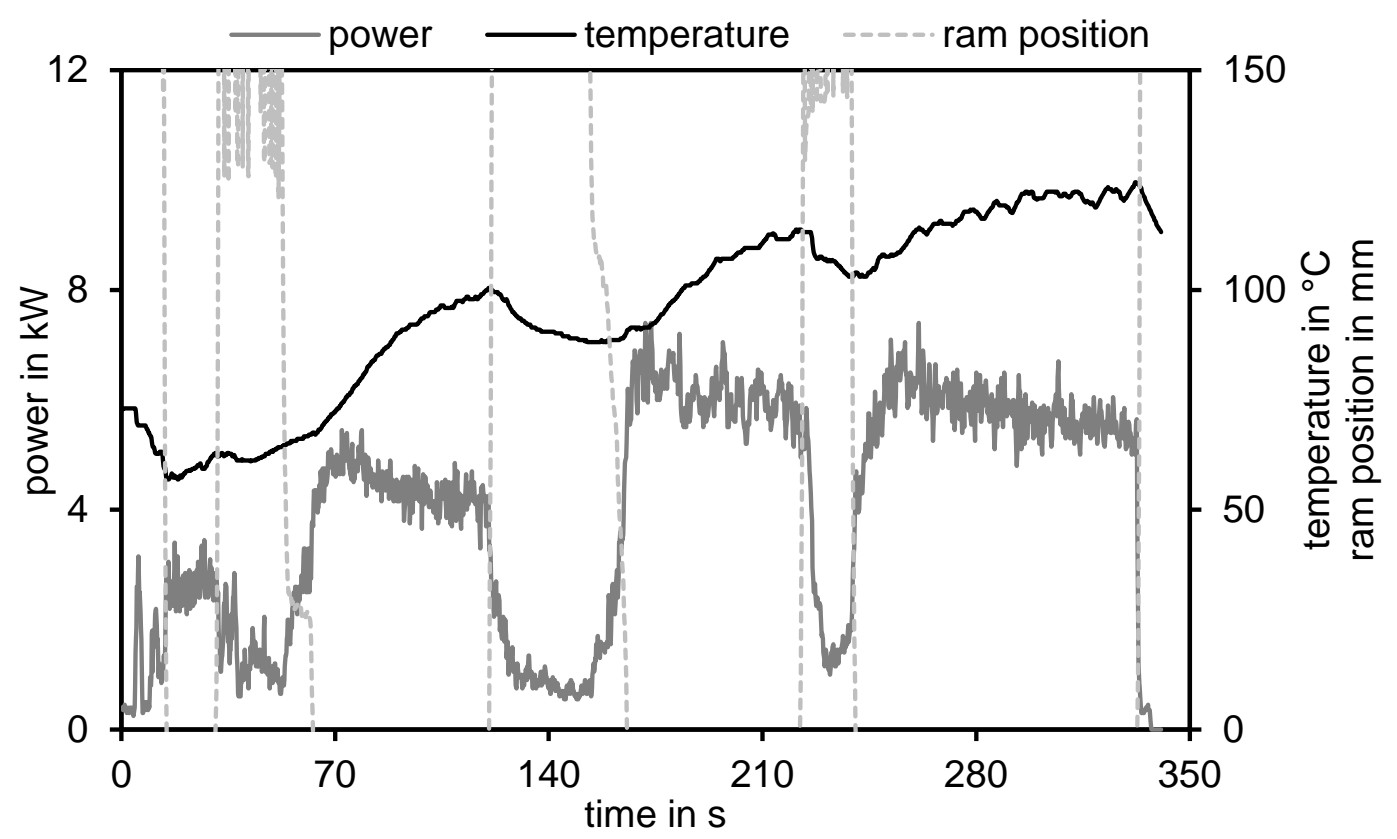

Figure 3.2: Fingerprint of the $1^{\text {st }}$ stage of the GT50 series

As can be seen the incorporation of the filler in two portions could be achieved but within a certain delay. Especially after adding the second portion of silica (time at $120 \mathrm{~s}$ ) the settling of the ram took $45 \mathrm{~s}$ in total. The average power during the last phase of mixing reached a value of roughly $6 \mathrm{~kW}$, which is significantly lower in comparison to the GT80 series (7 kW). The maximum temperature measured inside the mixing chamber did not reach the intended $130^{\circ} \mathrm{C}$. However, the temperature of all compounds were measured directly after mixing (dump temperature) by means of an external thermocouple and all values turned out to be in average 5 to $10^{\circ} \mathrm{C}$ higher than the measured temperature inside the mixer. Therefore, the mixing process to reach a suitable silanization of the silica was considered to be sufficient.

\section{Series 3 - NR55: NR formulation and mixing (55 phr of silica)}

The natural rubber formulation filled with $55 \mathrm{phr}$ of silica was chosen to be mixed in order to achieve a very narrow distribution of macro-dispersion results. Tab. 3.6 depicts the formulation for Series 3, NR55:

Table 3.6: NR formulation filled with 55 phr of silica (NR55)

$1^{\text {st }}$ stage

\begin{tabular}{llc}
\hline material & Type & phr \\
\hline SMR 10 (masticated) & Mooney 60 - 70 MU & 100.00 \\
Silica & variable & 55.00 \\
Si 266 & silane & adjusted to CTAB \\
ZnO RS RAL 844 C & zinc oxide & 3.00 \\
Edenor ST1 GS & stearic acid & 3.00 \\
Vulkanox HS/LG & TMQ protector & 1.00 \\
Vulkanox 4020/LG & 6PPD anti-aging & 1.00 \\
Protektor G 3108 & Wax & 1.00 \\
\hline \multicolumn{2}{c}{ 2 $^{\text {nd }}$ stage } \\
batch 1 & \\
\hline \multicolumn{2}{c}{ stage } & $3^{\text {rd }}$ stage \\
\hline batch 2 & \\
Rhenogran stage & & \\
Vulkacit CZ/EG-C & 80 \% DPG accelerator \\
Sulfur 80/90 & CBS accelerator \\
\hline
\end{tabular}


The formulation and mixing process were again divided into three stages and the amount of silane (Si266) was adjusted to the specific surface area of silica (CTAB). Therefore, the ratio of $5.0 \mathrm{phr}$ silane to $55 \mathrm{phr}$ of silica with a CTAB of $160 \mathrm{~m}^{2} / \mathrm{g}$ was used as a basis and the adjustment to the other types of silica respectively surfaces areas was made by the rule of three. Tab. 3.7 shows the mixing process for the NR55 series.

Table 3.7: Mixing process for the NR compounds filled with 55 phr of silica

stage and time

\begin{tabular}{|c|c|}
\hline $\begin{array}{l}1^{\text {st }} \text { stage } \\
\text { min:sec }\end{array}$ & $\begin{array}{c}\text { fill factor } 0.73 ; 70 \mathrm{rpm} \text {; chamber temperature: } 70^{\circ} \mathrm{C} \\
\text { measured temperature: } 130-150{ }^{\circ} \mathrm{C}\end{array}$ \\
\hline $00: 00-00: 30$ & polymer \\
\hline $00: 30-01: 30$ & 1/2 silica; silane \\
\hline $01: 30$ & cleaning step \\
\hline $01: 30-02: 30$ & 1/2 silica; TMQ; 6PPD; Wax \\
\hline $02: 30$ & cleaning step \\
\hline 02:30-04:00 & stearic acid; zinc oxide \\
\hline 04:00 & lift ram; aerate \\
\hline 04:00-05:00 & mixing \\
\hline \multirow[t]{3}{*}{ 05:00 } & dump and control temperature \\
\hline & $45 \mathrm{~s}$ on open mill ( $4 \mathrm{~mm}$ nip), sheet out \\
\hline & weigh compound for 2nd step; storage for $24 \mathrm{~h} / \mathrm{RT}$ \\
\hline $\begin{array}{l}2^{\text {nd }} \text { stage } \\
\text { min:sec }\end{array}$ & $\begin{array}{c}\text { fill factor } 0.70 ; 70 \mathrm{rpm} \text {; chamber temperature: } 80^{\circ} \mathrm{C} \\
\text { measured temperature: } 130-150^{\circ} \mathrm{C}\end{array}$ \\
\hline 00:00 - 03:00 & batch $1^{\text {st }}$ stage; \\
\hline 03:00 & dump and control temperature \\
\hline & $\begin{array}{l}45 \mathrm{sec} \text {. on open mill ( } 4 \mathrm{~mm} \text { nip), sheet out } \\
\text { weigh compound for } 3 \mathrm{rd} \mathrm{step;} \mathrm{storage} \mathrm{for} 4-24 \mathrm{~h} / \mathrm{RT}\end{array}$ \\
\hline $\begin{array}{l}3^{\text {rd }} \text { stage } \\
\text { min:sec }\end{array}$ & $\begin{array}{c}\text { fill factor } 0.68 ; 55 \mathrm{rpm} \text {, chamber temperature: } 50^{\circ} \mathrm{C} \\
\text { measured temperature: }>110^{\circ} \mathrm{C}\end{array}$ \\
\hline 00:00 - 02:00 & batch stage 2; accelerators; sulfur \\
\hline 02:00 & $\begin{array}{l}\text { dump batch; process on open mill } 20 \mathrm{sec} \text {. with } 3 \text { - } 4 \mathrm{~mm} \text { nip } \\
\text { cut } 3 \times \text { left, } 3 \times \text { right with } 3 \mathrm{~mm} \text { nip } \\
\text { roll up and pass through a } 3 \mathrm{~mm} \text { nip } \times 3 \\
\text { sheet off: store for minimum } 12 \mathrm{~h} \text { before vulcanization }\end{array}$ \\
\hline
\end{tabular}

Due to the relative low amount of silica of only $55 \mathrm{phr}$, it was decided to add the filler in two equal portions during the $1^{\text {st }}$ stage. Especially natural rubber is more sensitive to higher temperatures and has therefore be controlled more precisely [11]. Fig. 3.3 depicts the fingerprint of the $1^{\text {st }}$ stage of mixing for one type of silica (155P213) including the ram position, the power and the temperature profile as a function of time. The rotor speed was kept constant throughout the whole mixing. 


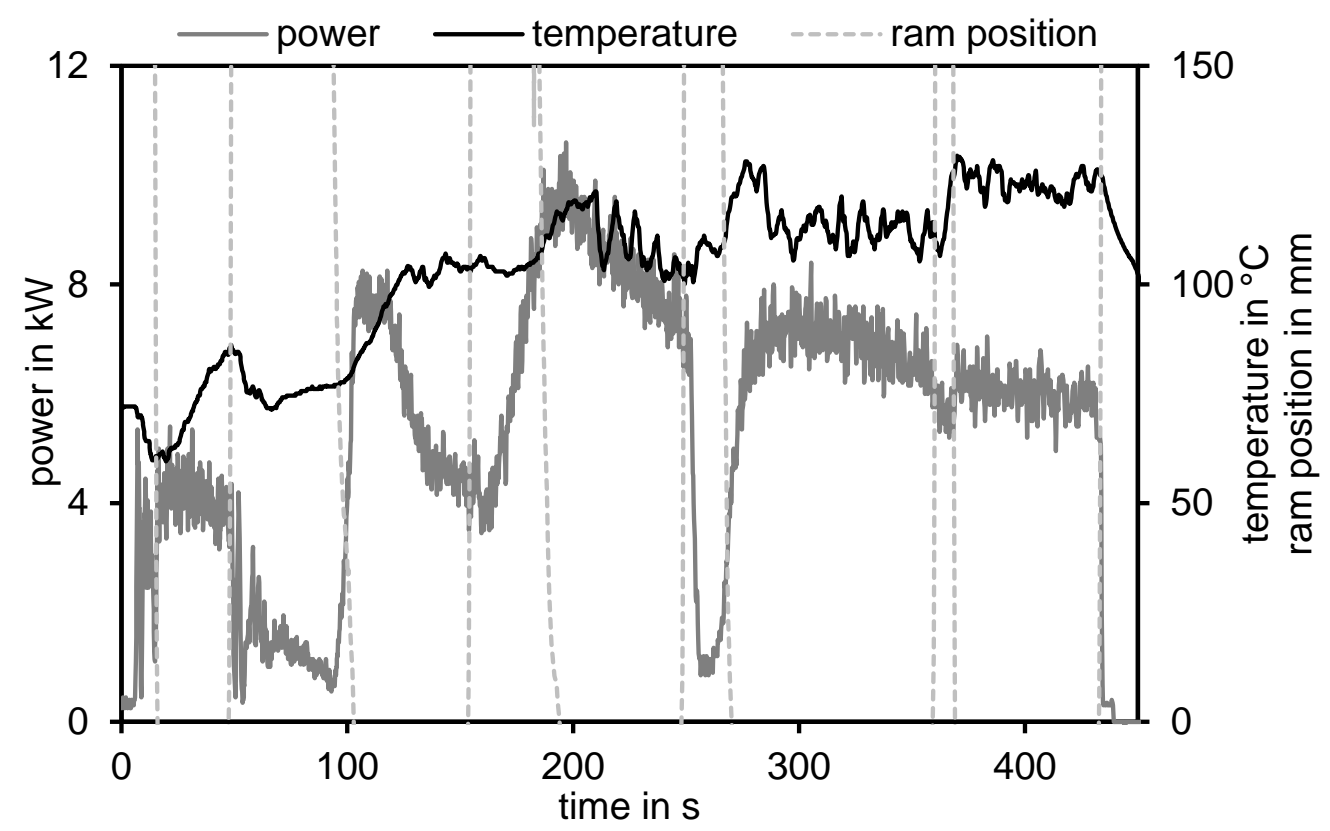

Figure 3.3: Fingerprint of the $1^{\text {st }}$ stage of the NR55 series

The filler was again incorporated in two portions. Especially the addition of the first portion of silica (time at $48 \mathrm{~s}$ ) took $45 \mathrm{~s}$. The average power during the last phase of mixing reached a value of roughly $8 \mathrm{~kW}$ and slowly dropped to $6 \mathrm{~kW}$ at the very end which might indicate a faster degradation of the natural rubber during mixing in comparison to synthetic polymers or a slower silanization reaction. The maximum temperature measured inside the mixing chamber did not reach the intended $130{ }^{\circ} \mathrm{C}$ as well but the measured dump temperatures were again roughly $10{ }^{\circ} \mathrm{C}$ higher. It can be expected that the mixing and therefore the silanization was sufficient while the temperature did not reach a critical value of $+160^{\circ} \mathrm{C}[11]$.

Series 4 - GT80 w/o: Green Tire formulation and mixing without silane (80 phr) As a final series, it was decided to mix a Green Tire formulation including 80 phr of silica but without silane (GT80 w/o). Therefore, the influence of the higher viscosity in this compound due to a higher filler-filler network on the one hand and the silane as a dispersion aid in the GT80 compound on the other hand can be discussed. Tab. 3.8 depicts the formulation for the GT80 w/o series. 
Table 3.8: Green Tire formulation filled with 80 phr of silica without silane (GT80 w/o) $1^{\text {st }}$ stage

\begin{tabular}{|c|c|c|}
\hline material & type & phr \\
\hline Buna VSL 4526-2 & oil-extended S-SBR & 96.25 \\
\hline Buna CB 24 & Nd-BR; cis1,4 > $96 \%$ & 30.00 \\
\hline Silica & variable & 80.00 \\
\hline N330 & carbon black & 5.00 \\
\hline ZnO RS RAL 844 C & zinc oxide & 2.00 \\
\hline Edenor ST1 GS & stearic acid & 2.00 \\
\hline Vivatec 500 & TDAE oil & 8.75 \\
\hline Vulkanox HS/LG & TMQ protector & 1.50 \\
\hline Vulkanox 4020/LG & 6PPD anti-aging & 2.00 \\
\hline Protektor G 3108 & Wax & 2.00 \\
\hline \multicolumn{3}{|c|}{$2^{\text {nd }}$ stage } \\
\hline \multicolumn{3}{|l|}{ batch $1^{\text {st }}$ stage } \\
\hline Rhenogran DPG-80 & $80 \%$ DPG accelerator & 2.50 \\
\hline \multicolumn{3}{|c|}{$3^{\text {rd }}$ stage } \\
\hline \multicolumn{3}{|l|}{ batch $2^{\text {nd }}$ stage } \\
\hline Richon TBZTD OP & TBzTD accelerator & 0.20 \\
\hline Vulkacit CZ/EG-C & CBS accelerator & 1.60 \\
\hline Sulfur $80 / 90$ & soluble sulfur & 2.00 \\
\hline
\end{tabular}

The chosen formulation as well as the mixing process is comparable to the GT80 series with the exception that the silane was fully left out. Therefore, only the type of silica was varied at a constant level of 80 phr. All other ingredients remained unchanged. The different dispersion qualities again should mainly be caused by the analytical properties of silica itself. Tab. 3.9 shows the mixing process for series 4 without the use of silane indicated as GT80 w/o. 
Table 3.9: Mixing process for the GT compounds filled with 80 phr of silica without silane

stage and time

\begin{tabular}{|c|c|}
\hline $\begin{array}{l}1^{\text {st }} \text { stage } \\
\text { min:sec }\end{array}$ & $\begin{array}{l}\text { fill factor } 0.73 ; 70 \mathrm{rpm} \text {; chamber temperature: } 70^{\circ} \mathrm{C} \\
\text { measured temperature: } 130-150^{\circ} \mathrm{C}\end{array}$ \\
\hline 00:00 - 00:15 & polymer \\
\hline 00:15 - 00:45 & 1/3 silica \\
\hline $00: 45-01: 15$ & 1/3 silica \\
\hline $01: 15-02: 15$ & $\begin{array}{l}\text { a) oil adsorbed on CB in a PE pouch } \\
\text { b) } 1 / 3 \text { silica } \\
\text { c) protector }\end{array}$ \\
\hline $\begin{array}{c}02: 15-04: 15 \\
04: 15\end{array}$ & $\begin{array}{l}\text { ZnO, stearic acid; Vulkanox HS; Vulkanox } 4020 \text {; } \\
\text { dump and control temperature } \\
45 \text { s on open mill ( } 4 \text { mm nip), sheet out } \\
\text { weigh compound for } 2 \text { nd step; storage for } 24 \text { h / RT }\end{array}$ \\
\hline $\begin{array}{l}2^{\text {nd }} \text { stage } \\
\text { min:sec }\end{array}$ & $\begin{array}{l}\text { fill factor } 0.70 ; 70 \mathrm{rpm} \text {; chamber temperature: } 90^{\circ} \mathrm{C} \\
\text { measured temperature: } 130-150^{\circ} \mathrm{C}\end{array}$ \\
\hline 00:00 - 01:00 & plasticize $1^{\text {st }}$ stage \\
\hline 01:00 - 03:00 & DPG; \\
\hline 03:00 & $\begin{array}{l}\text { dump and control temperature } \\
45 \mathrm{sec} \text {. on open mill ( } 4 \mathrm{~mm} \text { nip), sheet out } \\
\text { weigh compound for } 3 \mathrm{rd} \text { step; storage for } 4 \text { - } 24 \mathrm{~h} \text { / RT }\end{array}$ \\
\hline $\begin{array}{l}3^{\text {rd }} \text { stage } \\
\text { min:sec }\end{array}$ & $\begin{array}{c}\text { fill factor } 0.68 ; 55 \mathrm{rpm} \text {, chamber temperature: } 50{ }^{\circ} \mathrm{C} \\
\text { measured temperature: }>110^{\circ} \mathrm{C}\end{array}$ \\
\hline $\begin{array}{l}\text { 00:00 - 02:00 } \\
02: 00\end{array}$ & $\begin{array}{l}\text { batch stage } 2 \text {; accelerators; sulfur } \\
\text { dump batch; process on open mill } 20 \mathrm{sec} \text {. with } 3 \text { - } 4 \mathrm{~mm} \text { nip } \\
\text { cut } 3 \times \text { left, } 3 \times \text { right with } 3 \mathrm{~mm} \text { nip } \\
\text { roll up and pass through a } 3 \mathrm{~mm} \text { nip } \times 3 \\
\text { sheet off; store for minimum } 12 \text { h before vulcanization }\end{array}$ \\
\hline
\end{tabular}

Similar to the GT80 compounds it was necessary to add the silica in three equal portions, especially in case of powder materials. After each stage the compounds were sheeted out by the help of a two roll mill and stored for the specified time. Fig. 3.4 depicts the fingerprint of the $1^{\text {st }}$ stage of mixing for one type of silica (155P213) including the ram position, the power and the temperature profile as a function of time. The rotor speed was kept constant throughout the whole mixing. 


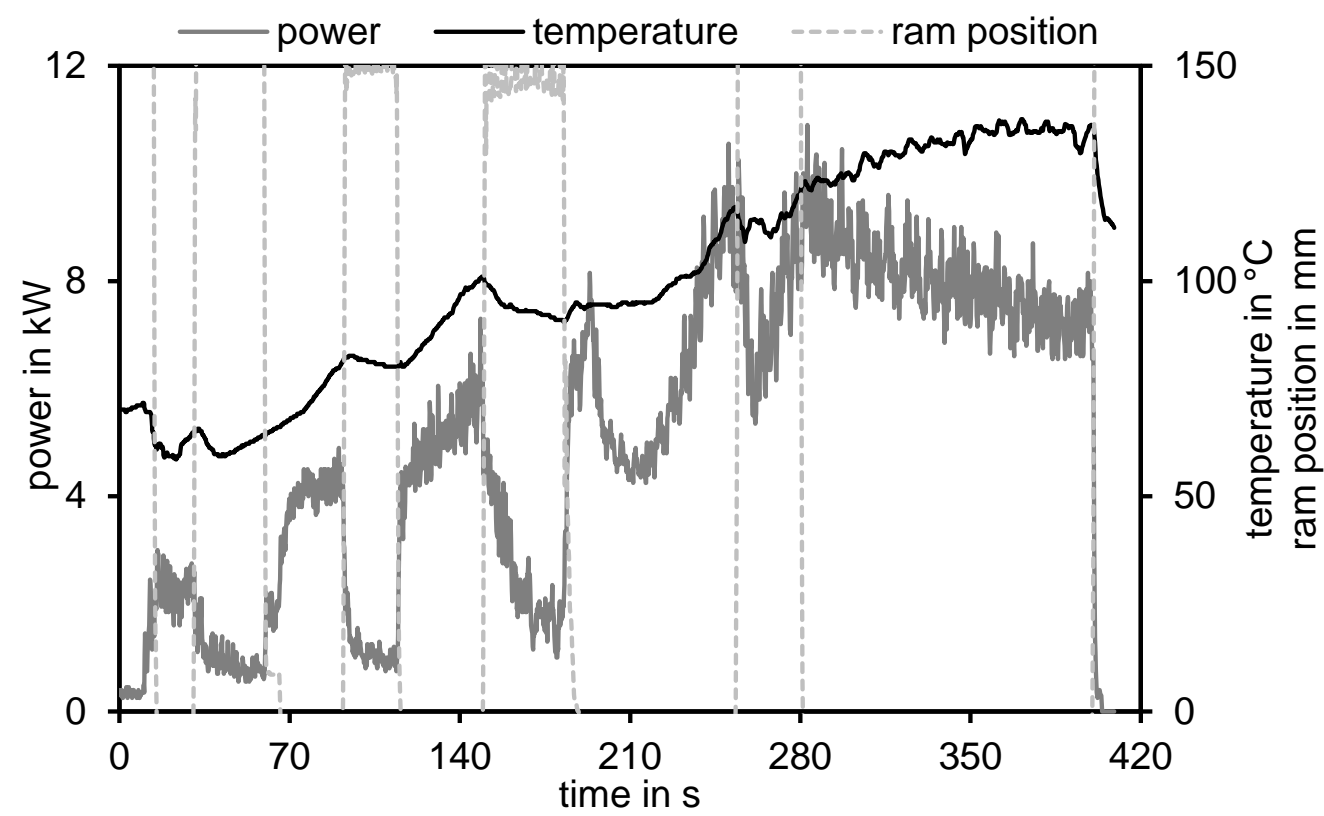

Figure 3.4: Fingerprint of the $1^{\text {st }}$ stage of the GT80 w/o series

All types of silica could be incorporated in three portions without facing major problems. Only during the addition of the third portion of silica (time at $150 \mathrm{~s}$ ) the settling of the ram was slightly delayed. It is noticeable that the power significantly dropped after the addition of the last ingredients (ZnO, stearic acid; Vulkanox HS; Vulkanox 4020). However, it reached almost the same value of $7 \mathrm{~kW}$ at the end of the mixing phase as the GT80 compounds with silane $(7 \mathrm{~kW})$. In the present case, the maximum temperature measured inside the mixer was roughly $135^{\circ} \mathrm{C}$. Without the use of low molecular weight silanes the compound viscosity is higher in comparison to the Green Tire 80 compounds with silane. A higher viscosity leads to higher shear forces and therefore increases the temperature of the compound.

To summarize, difficulties with the incorporation of silica respectively settling of the ram only occurred occasionally with the use of powders. All types of granules and micro-pearls in all four compound series were incorporated without any problems.

\subsection{Dispersion qualities}

The dispersion quality evaluations and the PAYNE-effect measurements are conducted with vulcanized rubber samples. Therefore, the optimum vulcanization times for each compound series were determined. The cure curves were determined by means of a moving die rheometer (MDR by Alpha Technologies) at an oscillating shear angle of $0.5^{\circ}$ and a frequency of $1.67 \mathrm{~Hz}$ in accordance with DIN 53529/ ISO 13145:2012 [12]. For each compound, the t95 time was evaluated. To keep to a consistent vulcanization time it was decided, to use the respectively longest t95 value within each series for all compounds within the same series. Following vulcanization conditions were finally chosen:

- Series 1-GT80: 15 min at $165^{\circ} \mathrm{C}$

- Series 2 - GT50: $13 \mathrm{~min}$ at $165^{\circ} \mathrm{C}$

- Series 3 - NR55: $17 \mathrm{~min}$ at $150^{\circ} \mathrm{C}$

- Series $4-\mathrm{GT} 80 \mathrm{w} / \mathrm{o}: 13 \mathrm{~min}$ at $165^{\circ} \mathrm{C}$

The samples for the Topography and Dispertester measurements were prepared as described in chapter 2.4.3 whereas the samples for the PAYNE-effect measurements were directly vulcanized before testing inside the Rubber Process Analyzer (Alpha RPA 2000). 


\subsubsection{Topography measurement}

The Topography measurement (Topo) as described in chapter 2.4.3 is a mechanical respectively tactile measurement system used in the present work to evaluate the macro-dispersion quality of all rubber compounds. In principle, two different values are evaluated, the peak area which corresponds to detected undispersed particles and the number of surface defects larger than $2 \mu \mathrm{m}$. The higher the peak area and the higher the number of surface defects larger than $2 \mu \mathrm{m}$, the more undispersed particles were detected, which means a worse macro-dispersion quality. Fig. 3.5 depicts the spread of both evaluation values of the Topography measurement for all four compound series.
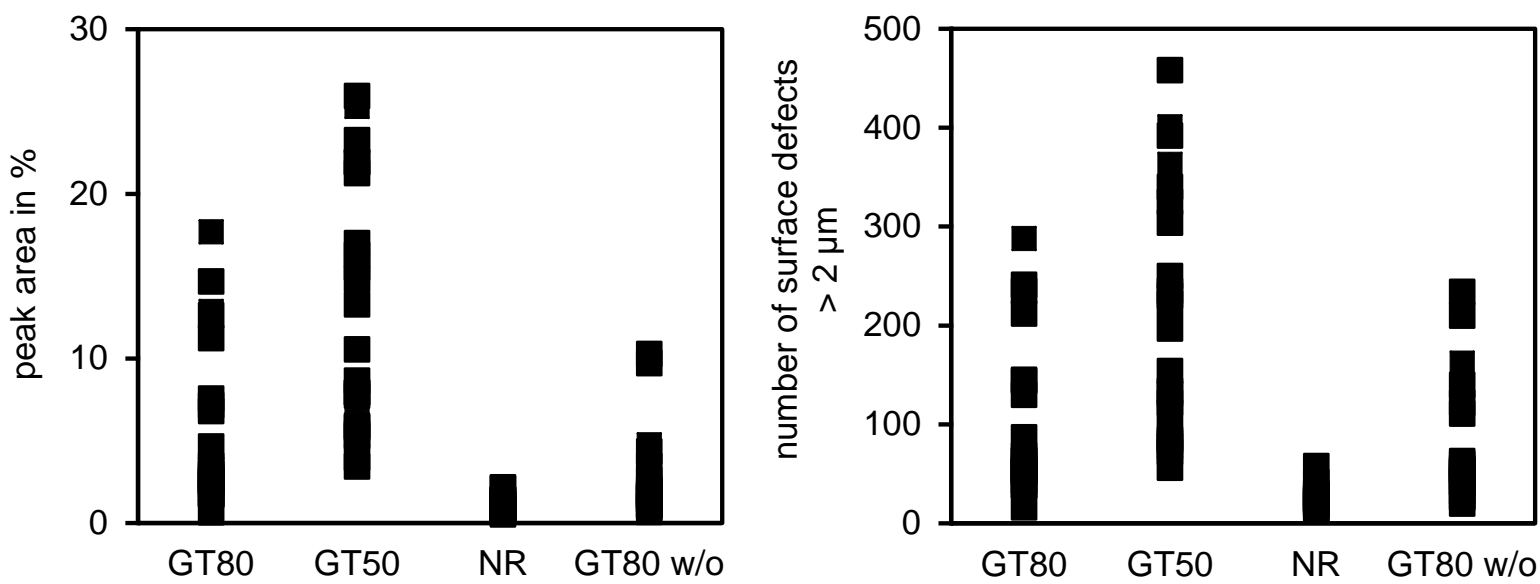

Figure 3.5: Range of different peak areas (left-hand side) and number of surface defects $>2 \mu \mathrm{m}$ (right-hand side) for all four compound series measured by means of the Topography Test

It can be seen that both evaluation values, peak area and number of surface defects $>2 \mu \mathrm{m}$, show the same tendency within each compound. As expected, the GT50 series lead to the widest spreading of results as well as the worst dispersion qualities due to the fact that the lower filler loading reduces occurring shear forces. Moreover, the NR series demonstrate the least differences and all over a very good level of dispersion qualities. The most surprisingly outcome of these measurements is the comparison of the GT80 with and without the use of silane. It was expected that the presence of silane functioning as a dispersion aid leads to an improved dispersion quality in comparison to the series without silane. However, the GT80 w/o compounds show improved macro-dispersion values in comparison with the GT 80 results. The use of silane with a relatively low molecular weight in comparison to the polymer leads to a lower viscosity of the compound due to a softening effect. A lower viscosity leads to lower shear forces and therefore decrease the effective dispersive mixing. As a conclusion, it can be said that the viscosity of the compounds seems to affect the macro-dispersion quality of silica to a higher extend than the use of silane as a dispersion aid. However, the use of silane might improve the micro-dispersion to a higher extend and lead to an all in all higher level of reinforcement.

\subsubsection{Dispertester measurement}

The Dispertester is an optical measurement system to evaluate the macro-dispersion of rubber compounds as described in chapter 2.4.3. All four compound series in the present work were investigated by means of this technique. As a result, the level of dispersion is given, the higher the dispersion value, indicated as maximum $100 \%$, the less undispersed particles were detected. Fig. 3.6 depicts the measured level of dispersion for all four compound series. 


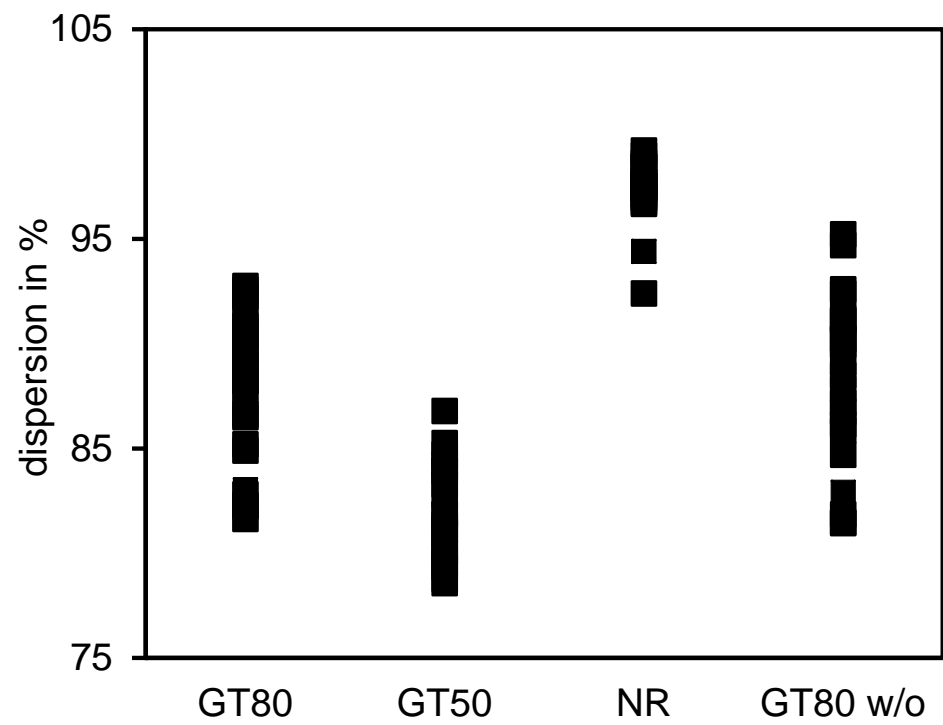

Figure 3.6: Range of different measured levels of dispersion for all four compound series measured by means of the Dispertester

Similar to the Topography measurements beforehand, the GT50 series possess the worst dispersion qualities whereas NR shows the best values. The result of both GT80 series are closer to each other but still indicating that the GT80 w/o compounds lead to a better macro-dispersion.

\subsubsection{PAYNE-Effect measurement}

It is stated in the literature that the PAYNE-effect can be used as an indirect measurement for the micro-dispersion [13]. Therefore, a strain sweep from $0.28 \%$ to $42 \%$ of deflection was measured by means of a Rubber Process Analyzer (Alpha RPA 2000) with a frequency of 1.6 Hz and at a temperature of $60{ }^{\circ} \mathrm{C}$. Beforehand, the samples were cured inside the measuring chamber in accordance with the determined vulcanization conditions. Two identical strain sweeps were performed directly after each other and the second one was evaluated between a maximum $G^{*}$ at $42 \%$ strain and a minimum $G^{*}$ at $0.28 \%$ strain. By means of the first strain sweep it was possible to reduce the effect of flocculation of fillers due to different storage times of the compounds. Fig. 3.7 depicts the results of the PAYNE-effect measurements for all four compound series.

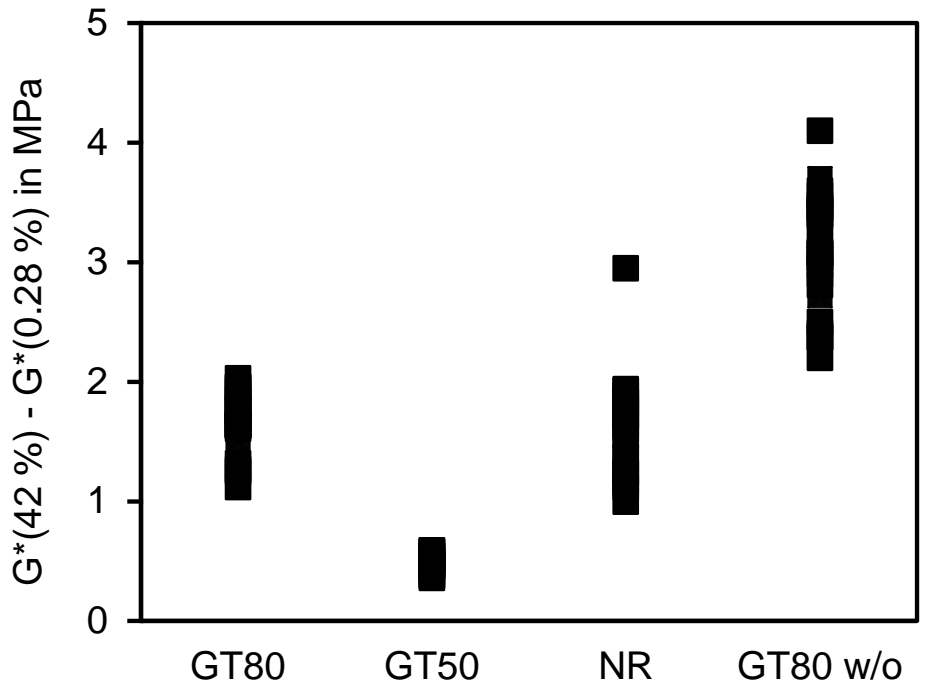

Figure 3.7: Range of different measured levels of the PAYNE-effect for all four compound series measured by means of the RPA

The first point to be recognized is the comparison between the Green Tire formulation (80 phr of silica) with (GT80) and without (GT80 w/o) the use of silane. The PAYNE-effect is strongly 
decreased by the use of silane due to the fact that the polar surface of silica is covered and therefore filler-filler interactions are reduced. This results in a less pronounced filler-filler network and hence in a reduced strain dependency of $G^{*}$. The Green Tire formulation filled with $50 \mathrm{phr}$ of silica possesses the lowest PAYNE-effect values which can be explained by the lower filler content and therefore lowest filler-filler network. These results strongly differ from the macro-dispersion measured by means of the Topography and Dispertester where e.g. the NR compounds demonstrated the best dispersion results. This indicates that the PAYNE-effect measurement is not a suitable method to evaluate the macro-dispersion quality.

\subsection{Special Investigations}

To get a deeper insight into the dispersion of silica, eleven selected samples of the first series GT80 were investigated by means of special methods. First of all, the results obtained by the Dispergrader were correlated with the outcome of the Dispertester measurements in order to compare two similar techniques with each other. Moreover, a confocal light microscope (CLM) was used to evaluate the dispersion quality within a wider size range. In addition, an X-ray computed microtomography $(\mu \mathrm{m}-\mathrm{CT})$ measurement system was used to obtain threedimensional information about the actual particle size and distribution inside a rubber compound. Finally, the abrasion resistance of different compounds were evaluated by means of the laboratory abrasion tester LAT100. These results are expected to possess a direct correlation to the in-rubber macro dispersion quality in chapter 5.

\subsubsection{Dispergrader measurement}

To compare two similar optical macro-dispersion measurement systems, series 1 (GT80) was investigated by means of the Dispertester (DisperTester 3000 Plus by MonTech) as well as the Dispergrader (DisperGRADER Alpha View by Alpha Technologies). Fig. 3.8 shows the range of measured dispersion results in \% (left-hand side) as well as the direct correlation of both measurement systems (right-hand side).
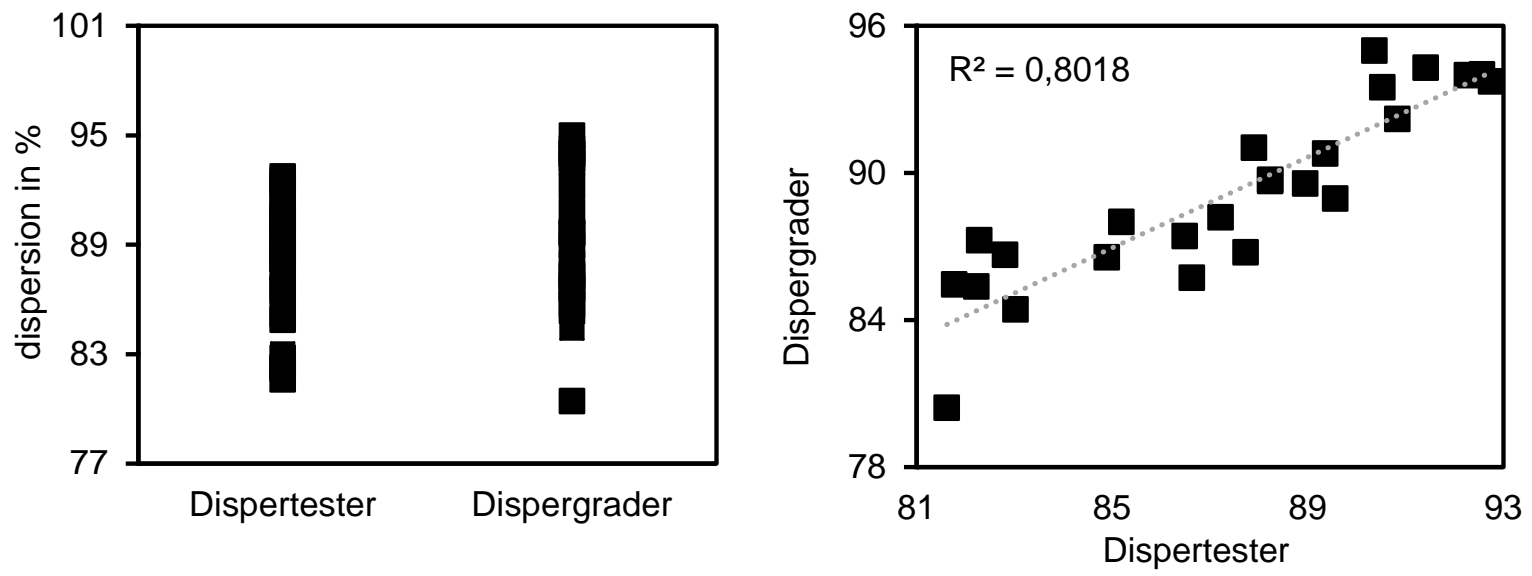

Figure 3.8: Range of different dispersion qualities (left-hand side) and direct correlation of the results (right-hand side) obtained from the Dispertester and Dispergrader of series 1 (GT80)

The obtained dispersion range of the Dispergrader measurements are slightly broader in comparison to the result of the Dispertester. Even with a similar measurement approach which in both cases is an optical measurement, the direct correlation of both systems shows noticeable differences. These deviations can be explained by different set-ups of the machines e.g. light sources, different evaluation algorithms of the evaluation software, not being able to measure the exact same spot of the sample and a general inhomogeneity of the rubber compounds itself. 


\subsubsection{Confocal light microscope (CLM)}

The confocal light microscope as described in chapter 2.4 .3 is a light optical measurement system to evaluate the dispersion quality of fillers inside a rubber matrix. By means of different lenses it is possible to evaluate the dispersion quality over a wider size range even to a rough sub-micron level (approximately $0.8 \mu \mathrm{m}$ ) in comparison to other optical systems. Therefore, eleven different compounds from series 1 (GT80) were investigated by this technique at a magnification of 10 and 100. Fig. 3.9 depicts the spreading of results for both resolutions as well as the linear correlation of the values.
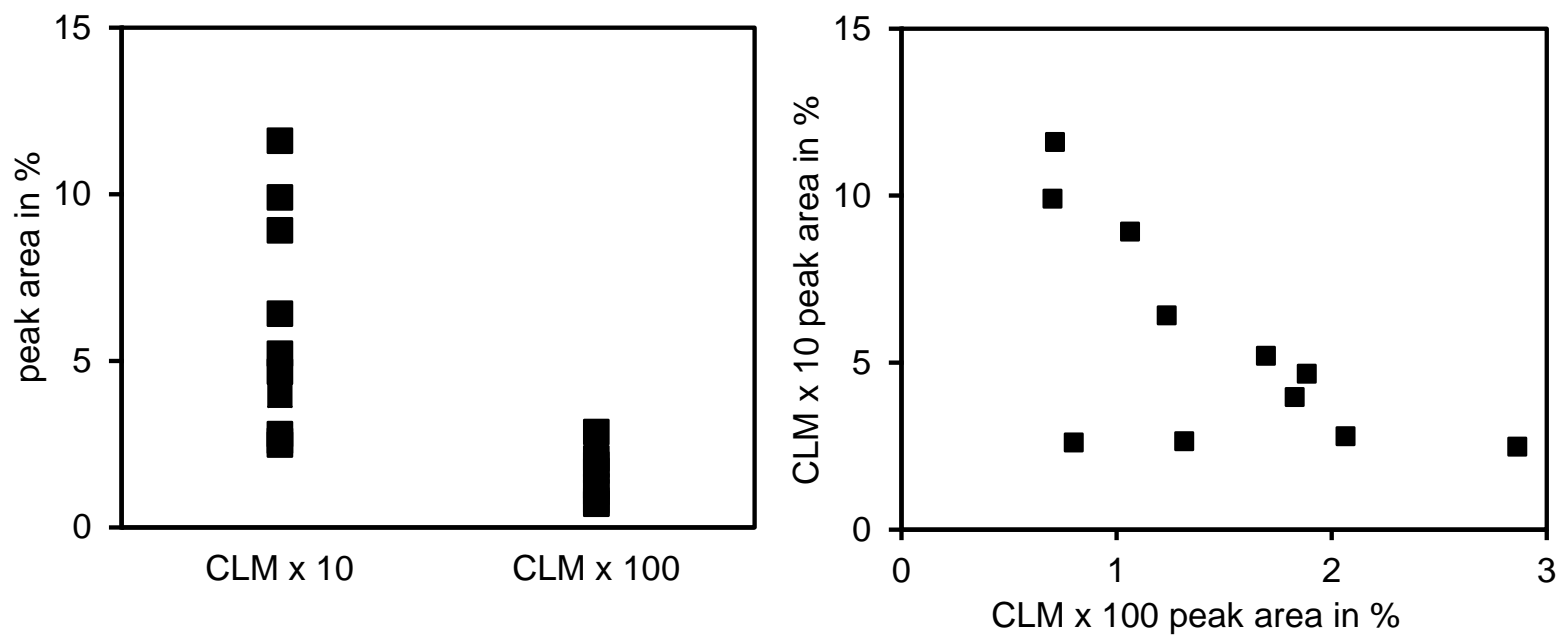

Figure 3.9: Range of different dispersion qualities (left-hand side) and direct correlation of results (right-hand side) from the CLM measurements at a magnification of 10 and 100

It can be seen that with a coarser resolution (CLM x 10) the dispersion qualities of the different samples spread widely in their results whereas with a finer resolution (CLM x 100) the dispersion qualities hardly differ. No linear correlation between both magnifications could be obtained which leads to the conclusion that the dispersion quality of fillers is not constant throughout each size range but clearly depends on the evaluated scale.

\subsubsection{X-ray computed microtomography ( $\mu \mathrm{m}-\mathrm{CT})$}

A new technique to evaluate the particle size distribution of fillers inside a rubber compound is the X-ray computed microtomography (Nanotom by GE Phoenix|x-ray) as described in chapter 2.4.3. In this way, it is possible to evaluate a bigger range of particle diameters and larger particles sizes in general [14]. To get a first indication about this system, four different compounds out of the series 1 (GT80) were investigated by means of this measurement. For the sample preparation, vulcanized samples were cut in a cylindrical shape containing a volume of roughly $110 \mathrm{~mm}^{3}$. Using this volume, it is possible to detect particles larger than $100 \mu \mathrm{m}$, however, the minimum size is limited to $15 \mu \mathrm{m}$. To investigate even smaller particles, the sample volume has to be reduced but then it is not possible anymore to detect larger clusters. Fig. 3.10 depicts the frequency of particles per volume as a function of their diameter of the four selected compounds whereby the 159GR204 sample was tested twice. 


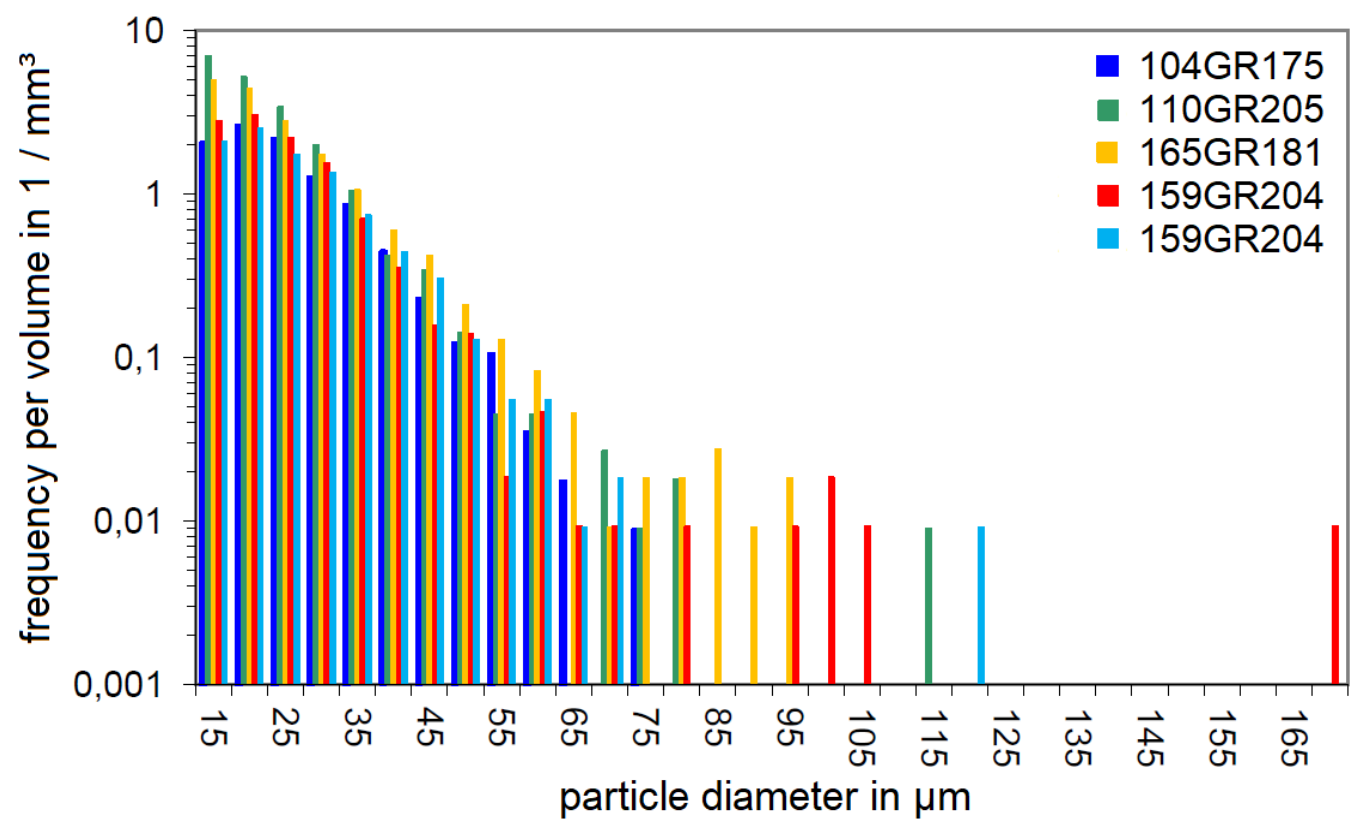

Figure 3.10: Detected frequency of particles per volume as a function of particle diameter
measured by means of the X-ray computed microtomography [15]

It is shown that a statistically relevant particle size can be found up to roughly $50 \mu \mathrm{m}$. Particles larger than that can still be detected but it is not possible to obtain reproducible results as seen when comparing both 159GR204 measurements e.g. for particles at 95, 100 and $105 \mu \mathrm{m}$ in diameter which are only detected in one compound. The compounds filled with 110GR205 and 165GR181 show the worst dispersion qualities respectively highest amount of particles in the area between 15 and $50 \mu \mathrm{m}$. The sample 104GR175 on the contrary shows the smallest amount of clusters and no single particles bigger than $75 \mu \mathrm{m}$ could be found [15]. It seems to be necessary to investigate a higher amount of samples to be able to judge the repeatability and in addition to use smaller samples as well for a finer resolution.

\subsubsection{Laboratory abrasion tester (LAT100)}

As stated in the literature [16-17] the macro-dispersion quality of fillers inside a passenger tire tread compound is related to the abrasion resistance. Therefore, one additional test method was taken into account to get an indication of this statement. A well-established method [18] to evaluate the abrasion resistance of tread compounds on a lab scale is the use of the laboratory abrasion tester LAT100. For this test, eleven selected compounds were vulcanized into the shape of a small wheel which were tested on a driving abrasive disc made out of corundum. During the measurement, the wheel was pressed to the disc at a constant load of $75 \mathrm{~N}$ whereas the speed of the disc and the slip angles were varied and the side force and friction were obtained. As a result, the abrasion loss over a wide range of speeds and energies was calculated [18]. The disc sharpness changes during the measurement, therefore the abrasion rate is usually evaluated as a relative rating in comparison to a standard compound as shown in eq. 3.1:

$$
\text { rel.rating }=\frac{\text { abrasion standard comp. }}{\text { abrasion experimental comp. }} * 100
$$

As a reference compound, the GT80 filled with the 159GR204 silica was chosen. In comparison to this, ten different compounds filled with silica granules were selected and tested. The relative abrasion ratings under low severity, high severity and in total were evaluated in accordance with GROSCH [19] relative to the reference compound which was set to $100 \%$, as shown in Tab. 3.10. The higher the rating, the better the abrasion resistance. 
Table 3.10: Evaluated abrasion ratings for low and high severity as well as in total obtained by means of the laboratory abrasion tester LAT100 for eleven different compounds

\begin{tabular}{cccc} 
Silica & $\begin{array}{c}\text { total rating } \\
\text { in } \%\end{array}$ & $\begin{array}{c}\text { low severity } \\
\text { in } \%\end{array}$ & $\begin{array}{c}\text { high severity } \\
\text { in } \%\end{array}$ \\
\hline 159GR204 & 100.0 & 100.0 & 100.0 \\
\hline 104GR175 & 92 & 90.8 & 87.8 \\
190GR197 & 98 & 93.8 & 103.9 \\
157GR186 & 99 & 97.0 & 99.7 \\
110GR205 & 88 & 88.9 & 86.0 \\
171GR196 & 105 & 100.7 & 108.2 \\
175GR203 & 105 & 103.8 & 106.3 \\
173GR196 & 100 & 96.9 & 103.4 \\
158GR209 & 109 & 105.6 & 110.2 \\
197GR199 & 104 & 100.8 & 108.4 \\
165GR181 & 98 & 95.3 & 98.8 \\
\hline
\end{tabular}

Especially the compounds with a lower CTAB surface area (104GR175 and 110GR205) possess a noticeable lower abrasion rating in comparison to the other samples. These results are correlated to the in-rubber macro-dispersion quality in chapter 5.

\subsection{Summary}

This chapter provides an overview of all silica used and rubber compounds mixed and tested within the frame of the present work. In total, 25 different types of silica with a variety of analytical parameters and dosage forms (powders, granules and micro pearls) were chosen. For all these fillers, the five standard analytical parameters were specified which are the surface area measured by CTAB and BET, the initial structure measured by DOA, the moisture content and the $\mathrm{pH}$-value. All types of silica were mixed into four different compounds. A standard Green Tire formulation filled with $80 \mathrm{phr}$ of silica, a Green Tire formulation filled with $50 \mathrm{phr}$, a natural rubber formulation (NR) filled with $55 \mathrm{phr}$ of silica and finally a Green Tire formulation with 80 phr but without the use of silane were evaluated. For all compounds, the macro-dispersion quality was investigated by means of the Topography Test (mechanical system) and the Dispertester measurement (optical system). In addition, the PAYNE-effect was measured to obtain an indication for the micro-dispersion.

As expected, the NR compounds in general show the best dispersion qualities whereas the GT50 shows the widest spreading of results. Surprisingly, the use of the GT80 without silane possessed slightly better dispersion results than the GT80 compounds with silane. Therefore, it is concluded that the effect of silane as a dispersion aid is less pronounced than the effect of higher shear forces due to a higher viscosity in the absence of silane to the macro-dispersion quality. However, the PAYNE-effect could be reduced significantly by means of silane and therefore a better micro-dispersion and reinforcement can be expected.

In addition to the mentioned tests, further special measurements were conducted. First of all, the GT80 series was investigated by means of the Dispergrader. In this way, the results of two similar macro-dispersion measurement systems, the Dispergrader and the Dispertester, could be compared. A direct correlation of both systems was possible but expressed some noticeable differences most likely due to differences caused by the evaluation of the results. Moreover, a confocal light microscope (CLM) was used to investigate selected samples. With this light optical measurement system it was possible to evaluate the dispersion quality of fillers over a wider size range even to a rough sub-micron level. It turned out that the use of different lenses respectively magnifications did not necessarily result in similar dispersion qualities. Another method taken into account was the X-ray computed microtomography. 
In this way it should be possible to investigate a full volume of a rubber sample and not only the surface to get information about the particle size and distribution. First results showed that it is necessary to investigate a higher amount of samples to be able to judge the repeatability before the results can be interpreted.

Finally, eleven selected rubber samples were tested by means of the laboratory abrasion tester LAT100 to obtain additional information about the abrasion resistance of the compounds.

All these results will be correlated to the analytical parameters obtained by standard tests as well as the newly developed methods (chapter 4) in the upcoming chapter 5.

\subsection{References}

[1] ISO 5794:2010 - Rubber compounding ingredients - Silica, precipitated, hydrated - Part 1: Non-rubber tests

[2] ISO 19246:2016 - Rubber Compounding Ingredients - Silica - Oil absorption of precipitated silica

[3] ISO 9277:2010 - Determination of the specific surface area of solids by gas adsorption - BET method

[4] ISO 787-2:1981 - General methods of test for pigments and extenders - Part 2: Determination of matter volatile at 105 degrees $C$

[5] ISO 787-9:1981 - General methods of test for pigments and extenders - Part 9: Determination of $\mathrm{pH}$ value of an aqueous suspension

[6] A. Wehmeier, "Entwicklung eines Verfahrens zur Charakterisierung der Füllstoffdispersion in Gummimischungen mittels einer Oberflächentopographie", 1998, Fachhochschule Münster, Münster

[7] ASTM D 2663 - 14:2014 - Standard Test Method for Carbon Black - Dispersion in Rubber, Test Method C - Microroughness Measurement with Profilometer

[8] ASTM D7723:2011 - Standard Test Method for Rubber Property - Macro Dispersion of Fillers in Compounds

[9] A. R. Payne, Effect of dispersion on dynamic properties of filler-loaded rubbers, Rubber Chem. Technol. 39 (1966) 365-374

[10] C. Hayichelaeh et al., Reinforcement of natural rubber by silica/silane in dependence of different amine types, Rubber Chem. Technol. 90 (2017) 651-666

[11] F. Röthemeyer; F. Sommer, Kautschuktechnologie, Carl Hanser Verlag, Munich (2013)

[12] ISO 13145:2012 - Rubber - Determination of viscosity and stress relaxation using a rotorless sealed shear rheometer

[13] A. I. Medalia, Reinforcement of Elastomers, Encyclopedia of Materials: Science and Technology (2001)

[14] K. G. Jago, X-ray computed microtomography of rubber, Rubber Chem. Technol. 85 (2012) 387-407

[15] H. El Maanaoui et al., Computertomographische Untersuchung zur Partikelgrößenverteilung, DIK Deutsches Institut für Kautschuktechnologie (2018)

[16] A. I. Medalia, Microscopic estimation of carbon black dispersion, Rubber Age (1965) 82

[17] S. Uhrlandt; A. Blume, Kieselsäure für den Grünen Reifen - Prozesse, Produkte, Eigenschaften, Kautsch. Gummi Kunstst. 54 (2001) 520-527

[18] M. Heinz; K. A. Grosch, A laboratory method to evaluate comprehensively abrasion, traction and rolling resistance of tire treat compounds, Rubber Chem. Technol. 80 (2007) 580-607

[19] K. A. Grosch, Rubber Abrasion and Tire Wear, Rubber Chem. Technol. 81 (2008) 470505 


\section{Chapter 4.1 - The Principles of Method Development and Sample Preparation}

The present chapter provides an inside into the principles of an analytical method development which forms the basis for all upcoming investigations. These principles can be divided into the purpose of the measurement, the requirements which have to be fulfilled by a new method, the variation possibilities of the instrument settings and the evaluation of the final results.

A special emphasize is put on the sample preparation. It is known that e.g. the moisture content and the dosage form can influence the final outcome of a measurement. Moreover, it is important to be aware of the assumptions and approximations made during a calculation when using a particle size distribution measurement system.

A common technique to reduce the cluster sizes of silica is the use of an ultrasonic device. A watery silica solution is thereby treated by sonification respectively cavitation. This effect is influenced by various parameters and represents one of the most sensitive sample preparation methods. Additionally, the wear of the sonotrode has to be taken into account as well.

\subsubsection{Introduction}

The development of new analytical test methods to investigate silica is a continuous endeavor. Therefore, a guideline is proposed to divide this complex task into four main basic principles. The first one is referred to as the purpose of the measurement. The product characterization of silica is commonly used for the quality control and to support the development of new products. The second principle is the definition of the requirements to be fulfilled by the new method which are mainly the repeatability and distinguishability of different types of silica. Additionally, an independency of storage effects and dosage form is requested as well as the possibility to use the method within a daily lab routine. The third category are the variation possibilities of the instrument settings and the sample preparation. The latter is one of the most influencing parameters during a method development and is therefore emphasized to a greater extend. The last principle takes the evaluation possibilities of the final results into account. The aim of these investigations is to determine the impact of varying machine parameters on the sensitivity, reproducibility and distinguishability of the test results.

After dealing with the basic principles of a method development in the first part of this chapter, the second part deals with the sample preparation itself. Depending on the type of measurement different silica characteristics have to be taken into account e.g. the moisture content and the dosage form. To reduce the cluster sizes of different types of silica, an ultrasonic device was used. A watery suspension was treated by means of sonification respectively cavitation to apply energy and overcome particle-particle interactions. This sample preparation method is one of the most sensitive ones and is influenced by various parameters. Especially the wear of the sonotrode has to be taken into consideration and therefore monitored on a regular base.

The present work comprised two different types of particle size measurement systems. For those it is necessary to be aware of the assumptions and approximations used to calculate the diameters of the particles. These values cannot be considered as absolute numbers but can only be compared relatively to each other.

\subsubsection{The basic principles of method development}

The first basis to be defined is the purpose of the measurement itself. In case of an analytical method for investigated silica the objective is the product characterization. On the one hand it can aim for quality control of the production process. On the other hand it is supposed to support the development of new products. In this way new insights into the chemistry and morphology of the filler can be gained. In addition, direct correlations between the analytical and in-rubber properties can be obtained. Ideally, it would be possible to predict certain impacts on final compound characteristics with a new method. 
The second principle of a method development is referred to as the requirements which has to be fulfilled by the analytical approach. First of all, the method has to have a proper repeatability and reproducibility of results independently of the operator. Secondly, it has to be able to distinguish between different types of silica. Ideally, the final outcome should be independent of storage effects and the dosage form. These aspects will be considered more in particular later on in this chapter. Another issue to be taken into account is the daily lab routine. Especially handling and duration of a method should be consistent with the amount and frequency of samples to be tested as well as the benefit of the results. Finally, it is necessary to monitor and control the method itself e.g. by calibration standards which can guarantee comparability of results over a long period of time.

The variation possibilities are the third basis of a method development which has to be taken into account. Those can be divided into the parameters like those which are already given from the machine software, those which have to be added manually or those which should be considered additionally and the sample preparation in advance. The latter has a particular importance and will therefore be discussed more in detail later on in this chapter. In case of the machine parameters it is necessary to be aware of sample properties which possibly have to be specified e.g. the density of silica. In addition, all parameters which influence the procedure e.g. measurement speed and their impact on the final results have to be investigated in order to achieve the most suitable test conditions. To start with, most methods provide a default setting from which on all parameters subsequently can be varied.

Finally, the evaluation of the final results have to be investigated. These values are either directly supplied by the measurement device or have to be determined individually. These investigations have to be conducted in parallel with the variation of machine parameters in order to check which results are more sensitive to changes within the measurement.

\subsubsection{Sample preparation}

The sample preparation is one of the most influencing and hence important steps when using and developing an analytical method. A variety of parameters has to be taken into account and therefore the following points are discussed in more detail:

- Drying of silica

- Ultrasonic treatment

o Influences on the ultrasonic sample preparation

o Cavitation process during sonification

o Measuring the wear of the sonotrode

- Influence of the dosage form

- Particle size measurement systems

\section{Drying of silica samples}

Precipitated silica in general contains roughly 4 to $7 w t . \%$ of free water which does not only differ from type to type but also from batch to batch [1]. Especially when the density of silica is used for calculations within an analytical method, these differences might affect the final outcome of the measurement. Several already existing methods like the DOA measurement [2] include either a pre-drying step to overcome these deviations or the moisture content is measured separately [3] and considered in the calculation.

\section{Ultrasonic treatment}

Influences on the ultrasonic sample preparation

The ultrasonic sample preparation is a very common technique used to reduce the size of silica samples inside a watery suspension for analytical measurements. Various parameters can influence the final outcome of the preparation. The most obvious settings are the sonification 
time and used amplitude of the sonotrode. Both directly correlate to the energy input applied to the sample [4]. However, a higher energy input does not automatically lead to e.g. a smaller particle size distribution. It could be observed that there is a certain possibility of re-agglomeration of particles due to ultrasonic treatment as well [5].

In addition to the setting, the equipment of the ultrasonic device itself can influence the final results as well. Even two generators with the same wattage from different manufacturers can affect the sample preparation. Therefore, it is necessary to be aware of the used type of generator inclusive maximum watt and the type of the sonotrode [4].

Finally, the position of the sonotrode inside the beaker might better be kept constant in order to guarantee an even mixing of the suspension and therefore a homogeneous sample.

\section{Cavitation process during sonification}

To reduce the cluster sizes of different silica samples an ultrasonic device is often used. This setup consists of a generator, a converter and a horn respectively sonotrode. The sonotrode thereby immerses into a suspension of silica and water. While turned on the sonotrode oscillates at a high frequency of $20 \mathrm{kHz}$ which is within the ultrasonic range. When liquids are treated with ultrasound a phenomenon named cavitation occurs resulting from high differences in pressure. Thereby, the static pressure reaches a value beneath the vapor pressure of the fluid, gas dissolves from the liquid and vapor bubbles are formed. These bubbles grow and implode (Fig. 4.1.1) within ms resulting in a locally high temperature of approximately $5000{ }^{\circ} \mathrm{C}$ and very high pressure.

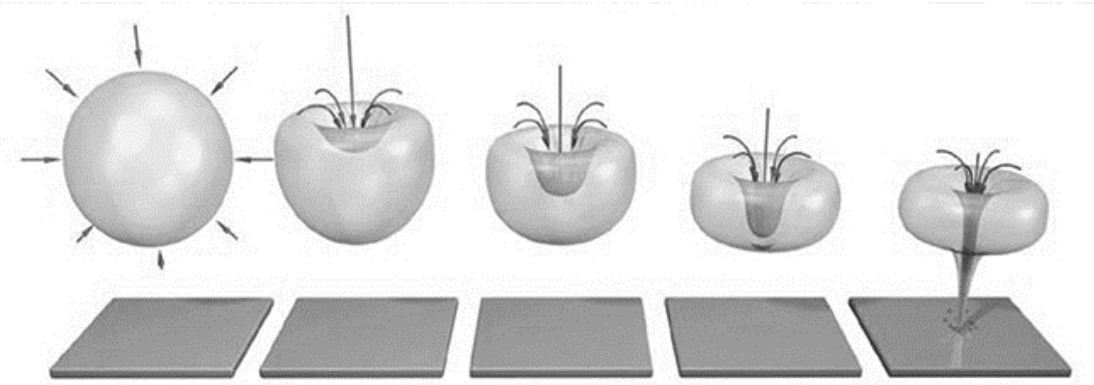

Figure 4.1.1: Schematic view of an imploding cavitation bubble [6]

Jet streams are formed at or nearby solid interfaces resulting in high shear forces which finally can reduce the cluster sizes of the silica sample. Inhomogeneities within the fluid, trapped air inside the sample and rough surfaces thereby support the formation of cavitations [4-5].

A simple experiment was conducted to observe where and how cavitation occurs during the sample preparation of silica. Two single pieces of silica granules were therefore put into a beaker filled with $50 \mathrm{ml}$ of demineralized water. At the beginning both pieces of granules settled to the bottom of the glass. A sonotrode was positioned in the center of the beaker with a distance of $20 \mathrm{~mm}$ to the bottom. When applying ultrasound with $70 \%$ of amplitude to the system the cavitation pressure leads to a flow and mixing of the suspension. This process was recorded by means of a high speed camera. Fig. 4.1.2 depicts three snapshots taken from this short movie. 
I

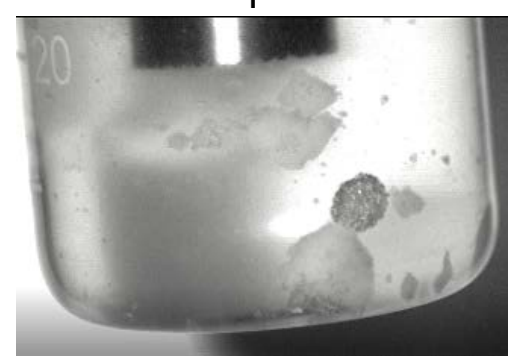

II

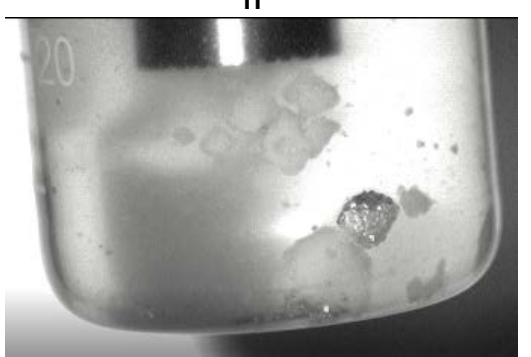

III

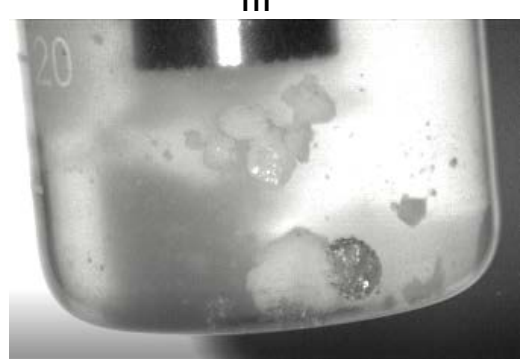

Figure 4.1.2: Impact of cavitation on two pieces of silica granules within a watery suspension

It is shown that after a short time of sonification (I) one granule was already dragged up by the occurred water flow into the direction of the tip of the sonotrode whereas the second granule still remained on the bottom of the beaker. Next to it, a trapped air bubble can be seen which however does not correspond to a cavitation bubble. With continuing time of the experiment (II) and (III) the upper granule starts to get fragmented whereas the lower particle still remains intact. This observation clearly indicates that cavitation only occurs directly underneath the tip of the sonotrode. Therefore, it is necessary to perform ultrasonic sample preparations for a decent period of time to guarantee a homogeneous treatment of the suspension.

As a conclusion it can be said that the ultrasonic sample preparation is one of the most sensitive sample preparation methods and all settings have to be precisely fulfilled. Otherwise, it is hardly possible to compare and reproduce final results. However, even if assuming that all parameters are determined, the wear of the sonotrode has to be taken into account as well.

\section{Measuring the wear of the sonotrode}

The wear of the sonotrode has a major impact on sample preparation by sonification and has to be therefore controlled regularly. The easiest approach is to visually inspect the sonotrode as can be seen in Fig. 4.1.3:

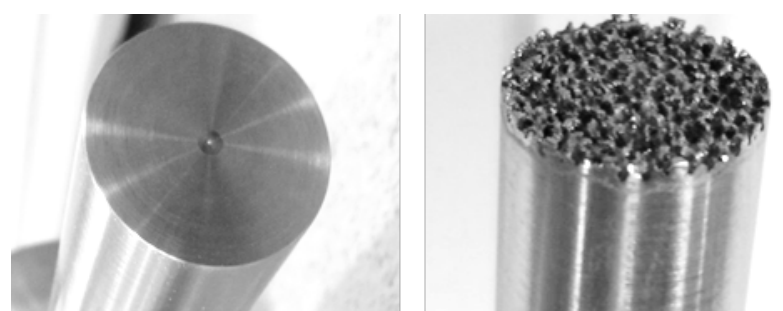

Figure 4.1.3: Pictures of new (left-hand side) and a worn (right-hand side) sonotrode

The figure on the left-hand side shows a new sonotrode with an even tip whereas an eroded tip is depicted on the right-hand side. These can just be judged qualitatively but no quantitative statement can be made. A worn-out sonotrode also tends to be noisier during sonification but again this cannot be evaluated scientifically [7].

A second approach to control the wear of the sonotrode is the use of a standard silica which is subsequently measured after sonification e.g. by a particle size distribution device. Deviations of the final results thereby can most likely be correlated to the change in sonification by the eroded sonotrode. However, choosing a suitable silica as a standard comprises certain risks: Highly dispersible (HD) types of silica are on the one hand in general less sensitive to the erosion of the sonotrode and changes in their particle size distribution can be detected only after intensive wear-off. The breakdown of conventional types of silica on the other hand results in a much broader particle size distribution and is therefore less reproducible. 
Another approach know from the literature [8] is to determine the energy input by means of a calorimetric measurement. A thermal imaging camera was used to detect the heat of a suspension during sonification. It is expected that the erosion of a sonotrode results in a change in the energy input and can therefore be detected indirectly. Fig. 4.1.4 depicts the change in temperature of the sonotrode and suspension over time.

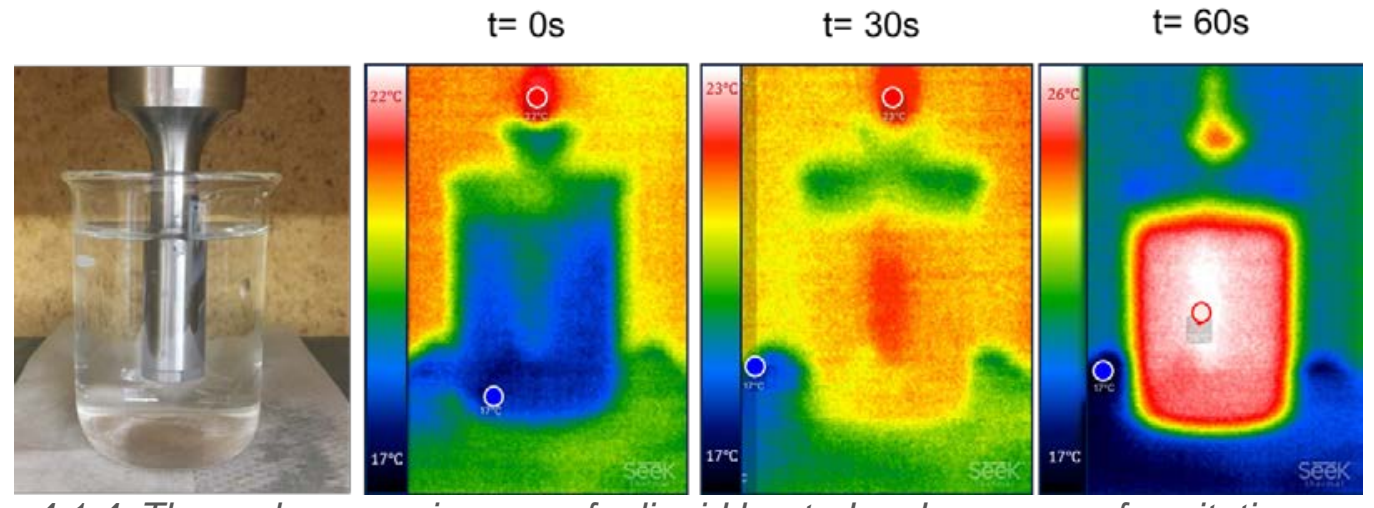

Figure 4.1.4: Thermal camera images of a liquid heated up by means of cavitation over time [8]

It can clearly be seen that the temperature rises with continuous sonification time. However, it is especially noticeable at 30 and $60 \mathrm{~s}$ that the hottest area is the whole sonotrode itself, not only at its tip where cavitation takes place. Therefore, it is most likely that the raise in temperature is due to the heat transfer from the sonotrode to the suspension and not due to the cavitation itself.

Rieger [5] tried to evaluate the energy input of the sonotrode by measuring the pressure of the cavitation. Therefore, a silica/water suspension with different concentrations was treated with ultrasound while the beaker was positioned on a scale. By subsequently increasing the amplitude the displayed weight increased. However, the measured deviations at higher amplitudes were too high to use this experiment as a suitable method to evaluate the energy input precisley.

A final approach to determine the wear of the sonotrode was to monitor the energy consumption respectively watt output of the generator. Therefore, three new sonotrodes and four worn-out ones were tested at $100 \%$ amplitude for 10 min (Branson Digital Sonifier 450D inclusive a flat $1 / 2$ " tip by BRANSON Ultrasonic Cooperation). $50 \mathrm{ml}$ of demineralized water was put inside a cooling bath $\left(5^{\circ} \mathrm{C}\right)$ to reduce the influence of the efficiency of the sonotrodes. The watt output displayed by the generator was recorded and plotted over time as shown in Fig. 4.1.5. 


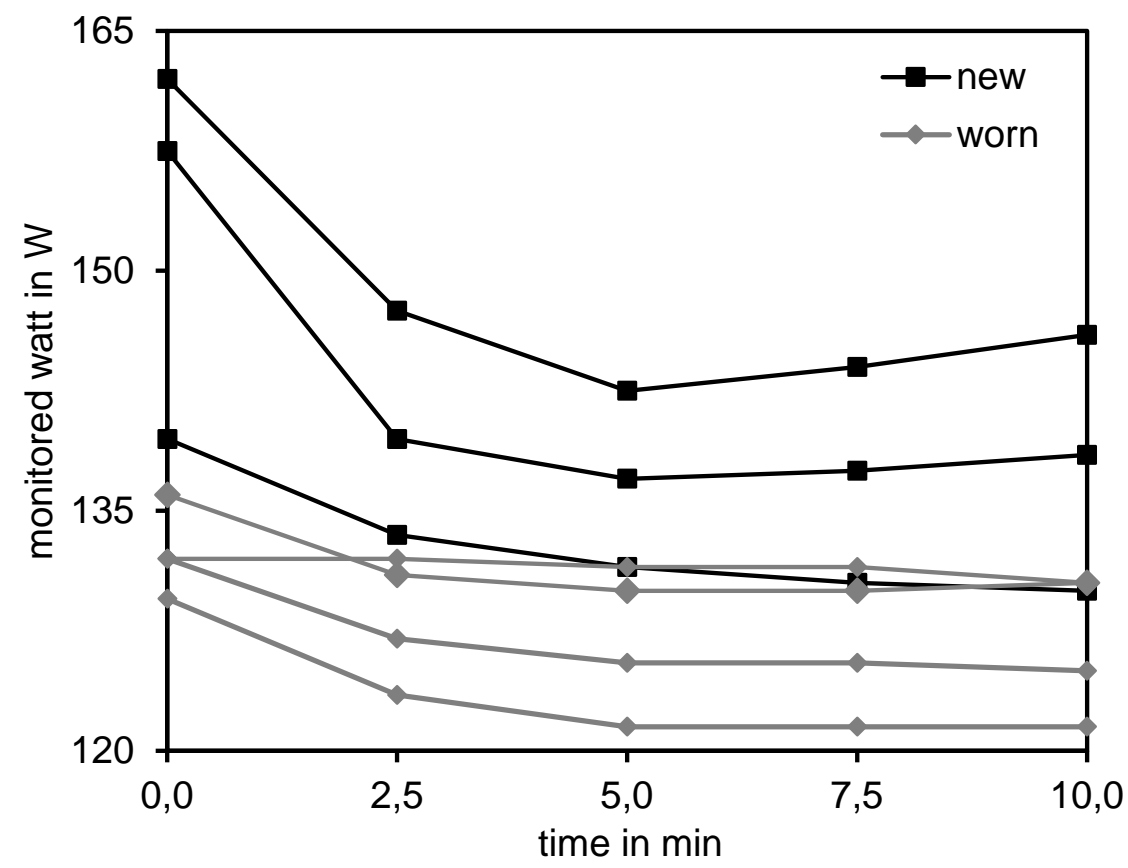

Figure 4.1.5: Monitored watt output of different new and worn sonotrodes over time

Almost all sonotrodes show a decrease in their watt output within the first minutes and subsequently reached a constant value. New sonotrodes thereby show a clear tendency to higher numbers in comparison to worn ones. However, the deviation of the three new ones is particularly high and one of them possess a comparable level to the old ones after 5 min sonification time. The direct comparison of the absolute watt values is therefore no suitable method to monitor the energy input. Heinemann [4] published a manuscript in which he discussed the watt output of ultrasonic generators and claimed also that this value is not reliable. A suitable solution could be to control the watt output for one sonotrode over time and evaluate a possible decrease due to erosion.

\section{Influence of the dosage form}

In the present work the DOA values [2] of the used types of silica vary in a wide range. However, powder samples in general seem to possess higher initial structure values $(196-238 \mathrm{ml} / 100 \mathrm{~g})$ in comparison to granules $(174-210 \mathrm{ml} / 100 \mathrm{~g})$. In addition, powders which were additionally granulated to obtain two types of dosages forms (related silica samples) always show a higher DOA value in their original powdery form as can be seen in Tab. 4.1.1:

\begin{tabular}{|c|c|c|c|c|c|}
\hline in $\mathrm{ml} / 100 \mathrm{~g}$ & Silica 1 & Silica 2 & Silica 3 & Silica 4 & Silica 5 \\
\hline Powder & 222.9 & 238 & 216.8 & 213 & 196 \\
\hline Granules & 195.8 & 203.4 & 196 & 204 & 181 \\
\hline
\end{tabular}

One explanation for the change in the DOA values could be that the initial structure of silica partly breaks down by applying high pressure to turn powder into more compact granules. Therefore, an additional experiment was conducted where three types of silica granules were milled by means of a rotor mill including an $80 \mu \mathrm{m}$ sieve [9]. In this way, the structure of silica should be further reduced and the obtained material is more similar to a powdery dosage form again. These milled samples were measured by means of the DOA method to evaluate its initial structure. Fig. 4.1.6 shows the DOA value of all three stages, the original powder, the granules and the milled material of three different silica. 


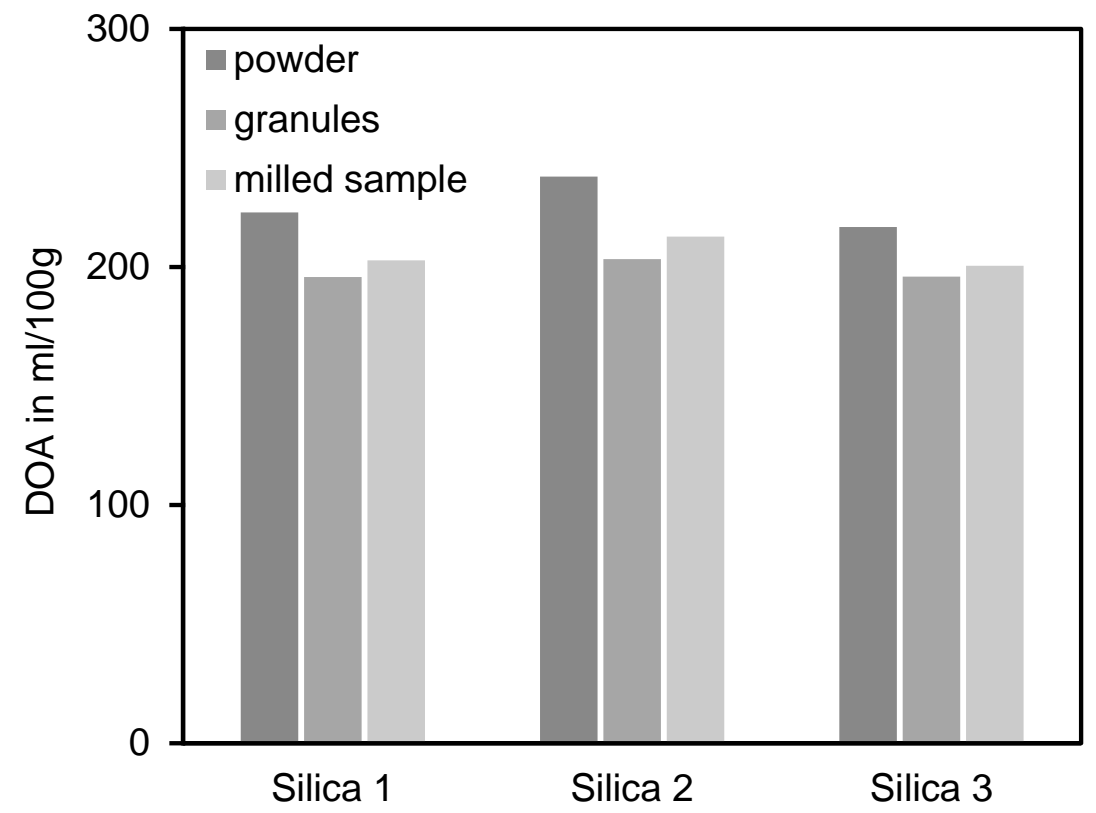

Figure 4.1.6: DOA values of 3 types of silica in their original form (powder), their related granules and the milled samples

Unexpectedly, it can be seen that the milled granules of all three samples show a higher DOA value than the granules itself. It is most unlikely that structure is rebuilt during the milling process. The conclusion which therefore can be drawn is that the measurement is indeed dependent on the dosage form and a comparison of different samples is to a certain extend limited.

\section{Particle size measurement systems}

In principle only one geometrical shape exists which can be described by a single uniform number which corresponds to a perfect sphere. In order to determine the actual particle size respectively diameter of a non-spherical shaped sample, it has to be clarified how to define the size. Therefore, an assumption has to be made to transfer the actual particle into a perfect sphere with a defined size which is referred to as the equivalent diameter. Even taking only a two dimensional particle into account various sizes can be measured respectively defined as depicted in Fig. 4.1.7 [10]:

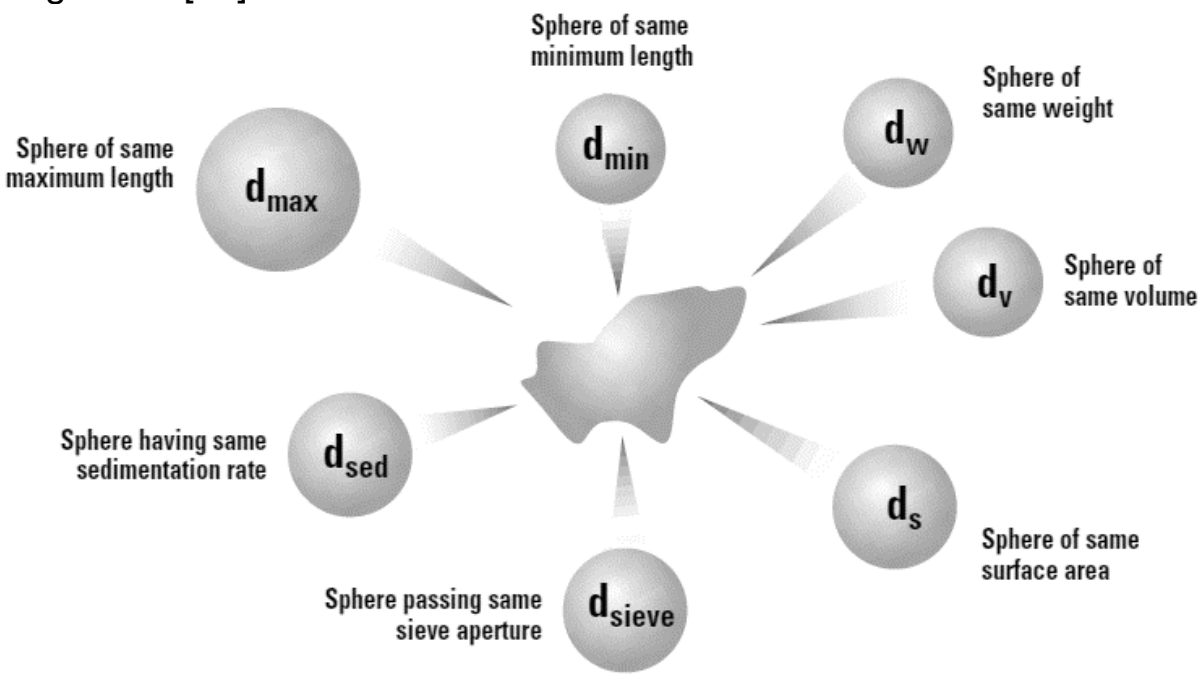

Figure 4.1.7: Schematic view of a non-spherical silica particle and the different possibilities to define its equivalent diameter [11] 
Depending on the used assumption the obtained diameter can vary significantly. As an example, a cylindrical particle with a height $h$ of $30 \mu \mathrm{m}$ and a diameter $d$ of $10 \mu \mathrm{m}$ is taken into account. This particle is measured with three different systems, a sieve aperture of $10 \mu \mathrm{m}$ mesh size, a measurement system which calculates the diameter on the basis of a sphere with the equivalent surface area and one system which is volume based. The actual volume $V_{c}$ of the cylinder thereby can be calculated as follows:

$$
V_{c}=\pi * r^{2} * h
$$

Where $r$ is the radius of the cylinder (half the diameter $d$ ) and $h$ is its real height in $\mu \mathrm{m}$. The surface $A_{c}$ can be expressed as:

$$
\boldsymbol{A}_{\boldsymbol{c}}=\mathbf{2} * \boldsymbol{\pi} * \boldsymbol{r} *(\boldsymbol{r}+\boldsymbol{h})
$$

The volume of a sphere on the other hand can be calculated by means of equation 4.1.3:

$$
V_{s}=\frac{4}{3} * \pi * r^{3}
$$

And its surface $A_{s}$ equals:

$$
A_{s}=\mathbf{4} * \pi * r^{2}
$$

Taken the actual volume $V_{c}$ respectively surface area $A_{c}$ into account and substituting it with equation 4.1.3 $\left(V_{s}\right)$ and equation 4.1.4 $\left(A_{s}\right)$, the equivalent volume diameter $d_{v}$ and the equivalent surface area diameter $d_{A}$ can be calculated. In addition, the sieve diameter $d_{S}$ can be obtained by means of a sieve aperture. Fig. 4.1.8 depicts the calculated particle sizes of these three different measurement systems:

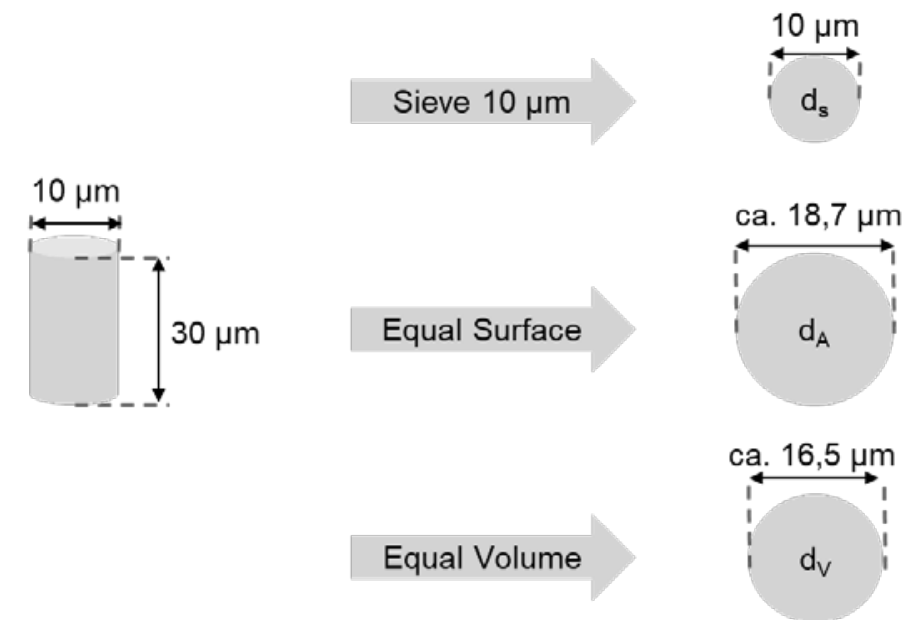

Figure 4.1.8: Schematic view of a cylindrical particle and evaluation of its equivalent diameter by means of a sieve, a surface and a volume based calculation [11]

It can be seen that the final outcome strongly depends on the used system and principle to obtain the particle diameter. Therefore, it is not possible to compare different particle size measurement systems with each other. Hence, the obtained numbers cannot be seen as absolute values. All results hence can only be compared relatively within the same measurement system. In addition to the different equivalent diameter, the calculation principle has to be taken into account. As an example, a scattering pattern obtained by using a laser diffraction system can be transferred into a particle size distribution curve by means of Fraunhofer or Mie approximation resulting in different values as shown in chapter 4.4. 


\subsubsection{Conclusion}

The analytical method development is a complex task. The basic requirements can thereby be divided into four different categories which are: The purpose of the measurement, either for quality and production control or to support the development of new products. The requirements an analytical method has to fulfil are a proper repeatability, distinguishability of different types of silica, an independency of storage effects and dosage form and it should be suitable for a daily lab routine. The variation possibilities include the parameters which can be adjusted by the device and the sample preparation. Finally, different evaluation possibilities have to be taken into account to compare the results.

One of the most important tasks is the sample preparation. For instance the moisture content of silica can influence the final result as well as the dosage form. When using a particle size distribution measurement system it is important to be aware of the assumptions and approximations used to calculate the diameters. These values can only be compared relatively to each other and it is not possible in general to compare results from different devices with each other. Cavitation by means of ultrasound plays a major role when a reduction of particle clusters is needed. Various parameters can thereby influence the ultrasonic sample preparations and the wear of the sonotrode has to be taken into account as well.

\subsubsection{References}

[1] R. K. Iler, The Chemistry of Silica, John Wiley \& Sons, New York - Chichester - Brisbane - Toronto (1978)

[2] ISO 19246:2016 - Rubber Compounding Ingredients - Silica - Oil absorption of precipitated silica

[3] ISO 787-2:1981 - General methods of test for pigments and extenders - Part 2: Determination of matter volatile at 105 degrees $C$

[4] A. Heinemann, Watt-Angaben beim Einsatz von Ultraschall-Desintegratoren (2005)

[5] J. Rieger, "Verfahrensentwicklung zur Partikelgrößenbestimmung gefällter Silica mittels Scheibenzentrifugen-Photosedimentometrie", 2016, Fachbereich Chemie- und Biotechnologie, Hochschule Darmstadt,

[6] https://www.theprocesspiping.com/introduction-to-cavitation/, date of access: 12.09.2018

[7] Internal Information, Evonik Resource Efficiency $\mathrm{GmbH}$

[8] R. R. Retamal Marín et al., Vergleichbarkeit der Dispergiervorschriften für die Charakterisierung von synthetischen amorphen Silicas, presented at: ProcessNet-FG „PMT“ Dresden (2017)

[9] Fritsch $\mathrm{GmbH}$, Advertising brochure: Rotor-Schnellmühle und Schlagkreuzmühle, (2016)

[10] M. Rhodes, Introduction to ParticleTechnology - Second Edition, John Wiley \& Sons, Southern Gate, Chichester, England (2008)

[11] Malvern Instruments Limited, Mastersizer 3000 User Manual (2013) 


\section{Chapter 4.2 - Method Development 1: Void Volume Structure Measurement}

To improve the abrasion resistance of silica filled passenger car tire tread compounds a good dispersion and distribution of the silica in the rubber matrix is required. From the literature it is known that the structure of fillers affects the dispersion process. The most common method to evaluate the initial structure of silica is the DOA measurement, whereby dioctyladipate (DOA) is absorbed by the void volume of a filler. Due to certain limitations of this DOA measurement an alternative method was investigated, the void volume structure measurement (VV).

The outcome of this measurement depends on the machine parameters, the sample weight and the sample preparation. Especially the moisture content of different grades of silica has to be taken into consideration by comparing results. Therefore, it is recommended to dry all samples beforehand.

The comparison of the void volume measurement evaluation with the DOA values result in a high correlation. Therefore, this newly developed method was successfully implemented to use it as a favorable alternative to the DOA measurement to describe the initial structure of silica.

\subsubsection{Introduction}

To develop new highly dispersible silica it is crucial to be aware of the typical analytical silica parameters and their impact on the dispersion process. It is known from the literature that the structure of fillers affects the in-rubber incorporation and dispersion process substantially as can be seen in chapter 2.4 [1]. Therefore, the present chapter focuses on the characterization and measurement of this analytical parameter. The initial structure of silica is usually determined by the DOA measurement whereby dioctyladipate (DOA) is absorbed by the void volume of a filler [2]. This is based on the assumption that a higher void volume indicates a higher initial structure and as a consequence a better dispersion behavior [3]. The DOA method is derived from the OAN (oil absorption number) which was developed for the investigation of carbon black [4]. The DOA number is influenced by the dosage form and the moisture content of a silica sample. Furthermore, this measurement system is limited by the degradation of the chamber and rotors over a period of time [5]. Additionally, working with oil is undesirable due to health issues, the time-consuming measurement and the cleaning process. To overcome these inconveniences an alternative method has to be taken into consideration.

In contrast to silica, there are different methods established to measure the structure of carbon black (CB). The most common technique is the determination of the OAN. To get more information about the stability of the CB structure the OAN was evolved into the COAN (compressed oil absorption number) where $\mathrm{CB}$ is pre-treated (compressed) before the actual measurement starts [6]. But there is no advantage in the measurement procedure itself, oil is still used. Due to the required extra step, the additional pre-treatment, this method is even more time-consuming. Additionally, silica cannot be investigated with this method due to the fact that the pre-treatment (compression) is not realizable [5]. A further development of the COAN is the calculation of the void volume of $\mathrm{CB}$ during a compression and decompression treatment inside a piston/cylinder system [7]. Previous works [8-9] show a linear correlation between the residual void volume of the decompression curve and the structure of CB (Fig. 4.2.1). The residual volume corresponds to the void volume value at atmospheric pressure after decompression, which is explained in more detail later in this chapter. 


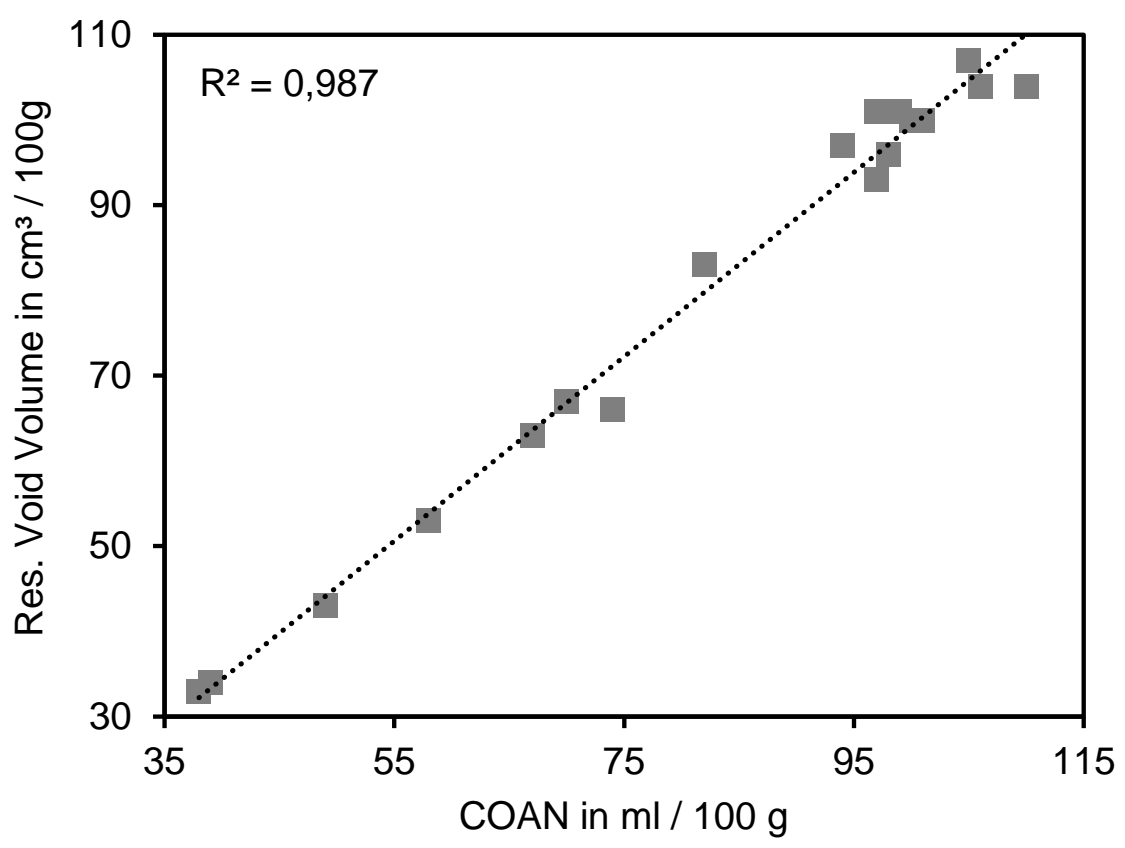

Figure 4.2.1: Linear correlation between residual void volume of carbon black and its COAN [9]

In the present chapter, silica was investigated with the above described void volume measurement system. Silica samples with different DOA numbers as well as different dosage forms (powders and granules) were tested. Characteristic values of the curve progression were evaluated and correlated to the DOA measurements. Finally, it was checked if the method meets the requirements for a newly developed method in accordance with what was claimed in chapter 4.1 .

\subsubsection{Theoretical principles}

The void volume measurement system (Compressed Volume Structure Tester by HITEC Luxembourg S.A.) consists of a cylinder, a piston (25.4 mm diameter) and a cover (Fig. 4.2.2). The piston and the cover include pressure sensors to report the applied and transmitted pressure during a measurement cycle. Additionally, the piston height (sample chamber height) was captured.

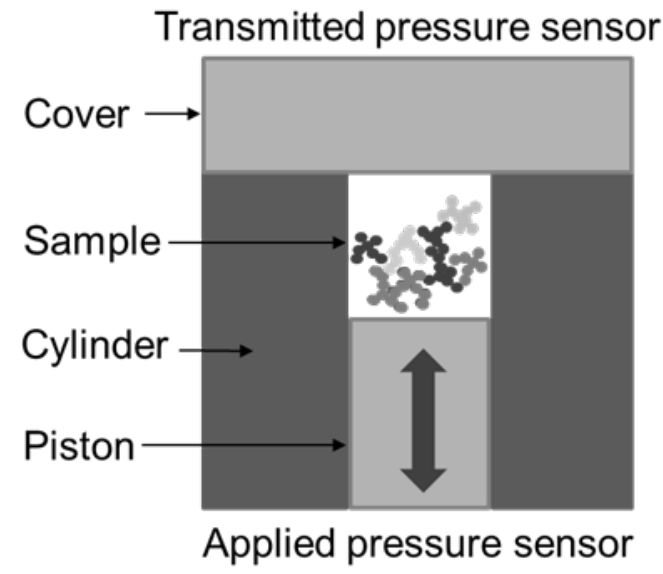

Figure 4.2.2: Schematic view of a void volume measurement system including filler sample 
With a known density $\rho$ in $\mathrm{g} / \mathrm{cm}^{3}$ (silica $=2.0$ [5] / carbon black $=1.8[10]$ ) and weight $m$ in $\mathrm{g}$ of the filler sample the theoretical volume $V_{T}$ in $\mathrm{cm}^{3}$ can be calculated by equation 4.2.1 [7]:

$$
V_{T}=\frac{m}{\rho}
$$

$V_{T}$ represents the absolute volume of the silica without any trapped air and pores. With a known cylinder diameter $D$ in $\mathrm{cm}$ and height of the sample chamber $h$ in $\mathrm{cm}$ the apparent compressed volume $V_{A}$ in $\mathrm{cm}^{3}$ can be calculated for each single pressure value by equation 4.2.2 [7]:

$$
\boldsymbol{V}_{\boldsymbol{A}}=\boldsymbol{h} * \boldsymbol{\pi} * \frac{D^{2}}{4}
$$

$V_{A}$ represents the total volume of the silica including trapped air due to the filler pores. The difference between the measured apparent volume $V_{A}$ and the theoretical volume $V_{T}$ results in the void volume $V V$ which correspond to trapped air inside the sample. The void volume (VV) was calculated per $100 \mathrm{~g}$ sample weight $(m)$ in $\mathrm{cm}^{3} / \mathrm{g}$ using equation 4.2 .3 [7]:

$$
\boldsymbol{V} \boldsymbol{V}=\frac{\left(\boldsymbol{V}_{A}-\boldsymbol{V}_{T}\right)}{\boldsymbol{m}}
$$

Fig. 4.2.3 depicts a typical carbon black void volume curve progression evaluated in accordance to international standards [7]. Due to friction loss there is always a difference between the measured applied and transmitted pressure. Therefore, the mean of both values is taken into account. The void volume was calculated for each single pressure value of the compression and decompression phase. The area between both curves corresponds to the dissipated energy (work) during the measurement. The carbon black sample expands while decompressing and stabilizes finally when atmospheric pressure is reached. The point where the curve hits the $y$-axis for the first time is determined as the residual void volume.

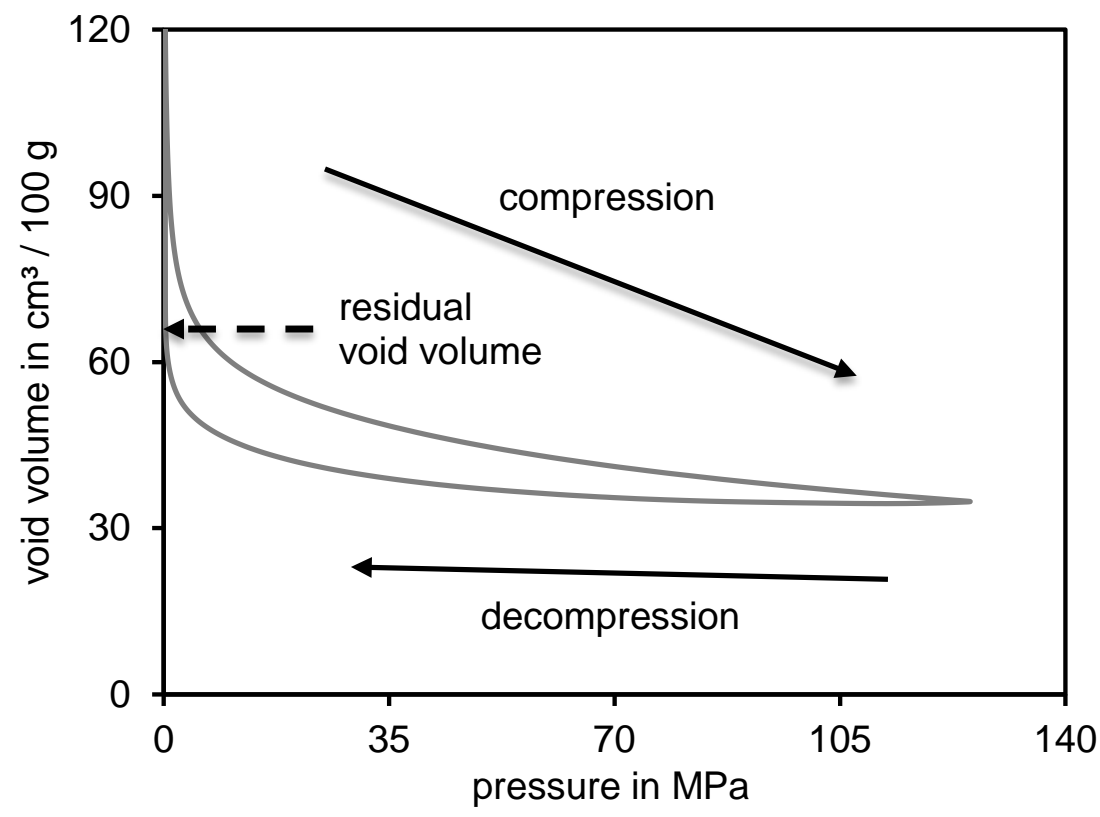

Figure 4.2.3: Typical carbon black void volume curve 


\subsubsection{Method Development \\ Carbon Black investigations}

In order to obtain a reference for the silica samples, three different types of carbon black (N326 / N330 / N339) were examined using void volume measurements. The default measurement setting in accordance with the standard was used [7]. $2.00 \mathrm{~g}$ of a filler sample were compressed with a default pressure rate of $2 \mathrm{MPa} / \mathrm{s}$ up to a target pressure of $125 \mathrm{MPa}$. Subsequently, the pressure was decreased with a constant rate of $2 \mathrm{MPa} / \mathrm{s}$ to atmospheric pressure. To avoid additional influences on the final result due to different moisture contents all samples were pre-dried for $2 \mathrm{~h}$ at $105^{\circ} \mathrm{C}$. A series of samples with different structures (OAN and COAN) and slightly varying external surface areas (STSA [11]) were chosen (Tab. 4.2.1).

\begin{tabular}{|c|c|c|c|c|}
\hline $\begin{array}{c}\text { carbon black } \\
\text { grade }\end{array}$ & $\begin{array}{c}\text { STSA [11] } \\
\text { in } m^{2} / g\end{array}$ & $\begin{array}{c}\text { OAN [4] } \\
\text { in } \mathrm{cm}^{3} / 100 \mathrm{~g}\end{array}$ & $\begin{array}{c}\text { COAN [6] } \\
\text { in } \mathrm{cm}^{3} / 100 \mathrm{~g}\end{array}$ & $\begin{array}{l}\text { residual VV [7] } \\
\text { in } \mathrm{cm}^{3} / 100 \mathrm{~g}\end{array}$ \\
\hline N326 & 77 & 72 & 69 & $70 \pm 0.2$ \\
\hline N330 & 76 & 102 & 88 & $93 \pm 0.3$ \\
\hline N339 & 88 & 120 & 99 & $107 \pm 0.3$ \\
\hline
\end{tabular}

Fig. 4.2.4 depicts the void volume measurements of N326, N330 and N339. Every measurement was repeated three times. The curve progressions of the repeated measurements run precisely on top of each other. The types of carbon black differ in their void volume at every pressure value which can be seen especially at the maximum value of $125 \mathrm{MPa}$. The higher the measured structure (OAN and COAN) the higher the calculated residual void volumes.

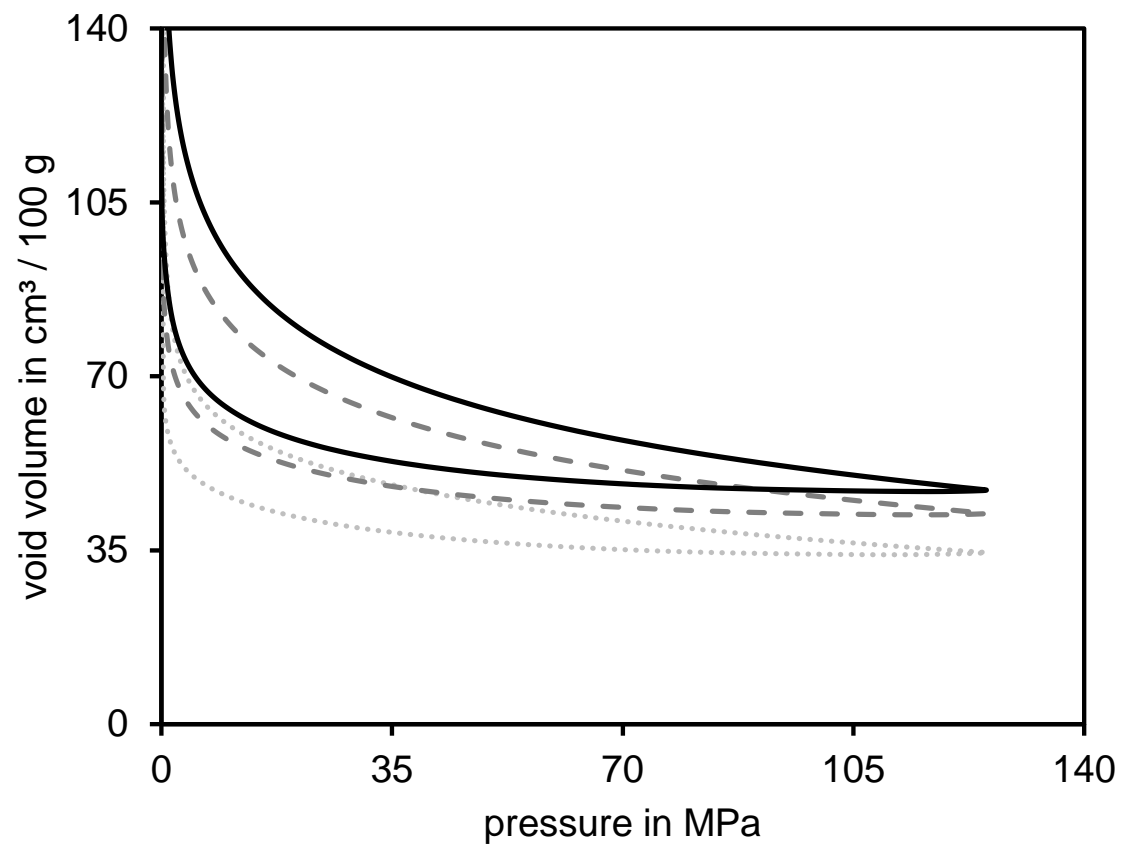

Figure 4.2.4: Void volume measurements of three different carbon blacks N339 (--), N330 (- - ) and N326 ( ..)

To confirm what was shown in Fig. 4.2.1 [9] a comparison of the COAN and the residual void volumes of the three measured CBs was conducted. Fig. 4.2 .5 confirms a linear correlation with a coefficient of determination $\mathrm{R}^{2}$ of 0.99 . 


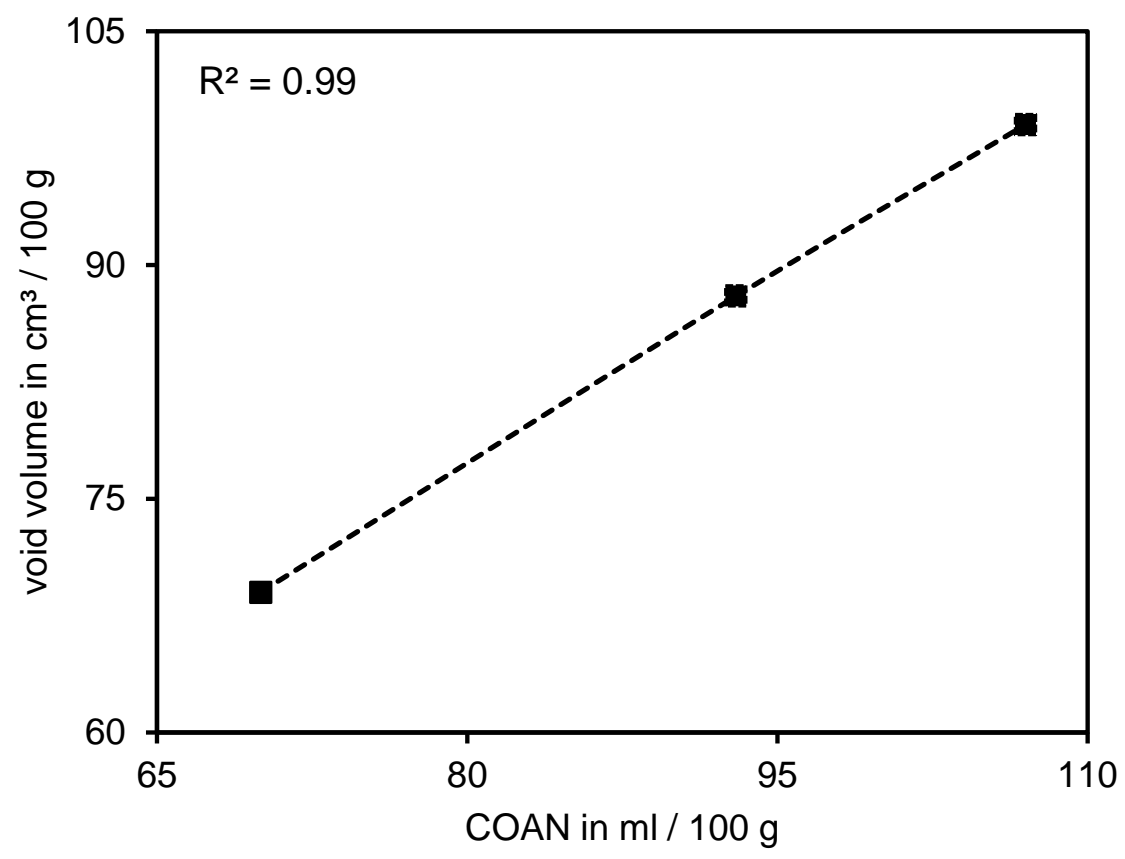

Figure 4.2.5: Correlation of COAN vs. residual void volume of $C B$

As a first result the void volume measurement can be judged as a suitable method to describe the structure of carbon black. It is able to distinguish between different types of CB and has an appropriate repeatability. The correlation between the COAN structure and the residual void volume measurements known from literature [8] is valid for the three tested CB samples as well. In the next step it was tested if this method can be transferred to silica.

\section{Silica investigations}

In order to develop a suitable method for the characterization of different types of silica the basic principles as described in chapter 4.1 have to be taken into account. Following this, it is necessary to be aware of the parameters which can be varied namely the machine settings on the one hand and the sample preparation on the other hand. The following list summarizes the investigations which were conducted:

- Default settings following carbon black measurement settings

- Influence of the moisture/water content (free water) inside the material

- Influence of the drying time

- Influence of the fines of granules

- Variation of the weight of the sample

- Variation of the piston speed: pressure-controlled

- Variation of the piston speed: path controlled

- Repeatability and reproducibility

- Storage test

\section{Default Settings}

A variety of silica samples were investigated with the void volume measurement system. As a starting point the default settings were chosen following the carbon black measurement without an additional pre-drying step:

- $\quad$ Sample weight:

- compression:

$2.00 \mathrm{~g}$

- decompression:

pressure rate of $2 \mathrm{MPa}$ / s up to a target pressure of $125 \mathrm{MPa}$ pressure decreased with a constant rate of $2 \mathrm{MPa} / \mathrm{s}$ to atmospheric pressure 
Firstly, the repeatability in general was tested. Fig. 4.2.6 depicts the void volume curve for one type of silica (104GR175) measured five times. Similar to carbon black, all measurement curves run on top of each other. Hardly any expanding during the decompression could be recognized which means that silica remains in the compressed state after pressure was applied. This behaviour can be explained by the high surface polarity of silica due to the high amount of silanol groups at its surface. While being compressed these silanol groups form additional strong hydrogen-bonds which inhibit the decompression. At a certain point, the silica sample gets stuck at one side of the sample chamber whereas it is released from the other side. Therefore, one sensor still records a certain pressure while the other one does not. As a consequence, the void volume rises without reaching atmospheric pressure and the residual void volume cannot be evaluated.

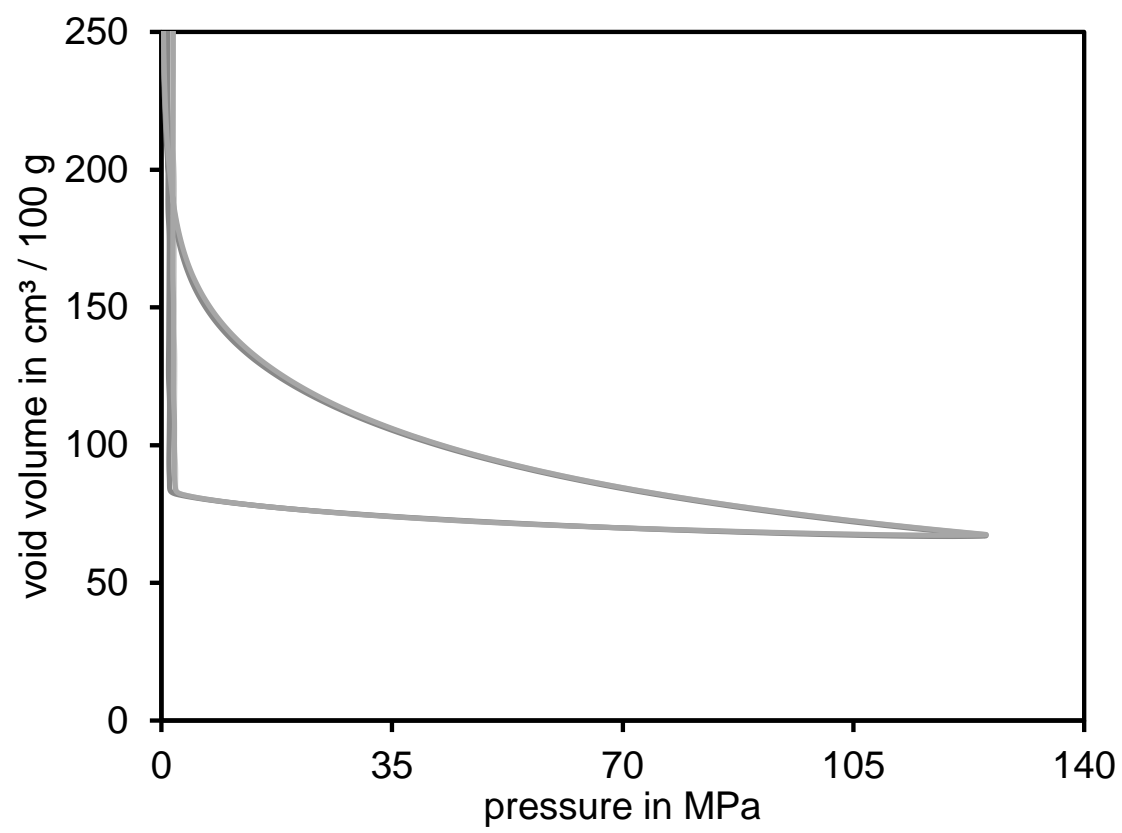

Figure 4.2.6: Void volume measurement of one type of silica (104GR175) measured five times

Secondly, different types of silica and dosage forms were investigated in order to evaluate their influence on the measurement. Fig. 4.2.7 shows two different silica granules (104GR175, 158GR190), one silica powder (169P202) and one micro-pearl (157MP207). As mentioned before, the decompression cannot be evaluated, therefore only the compression curve is taken into consideration. 


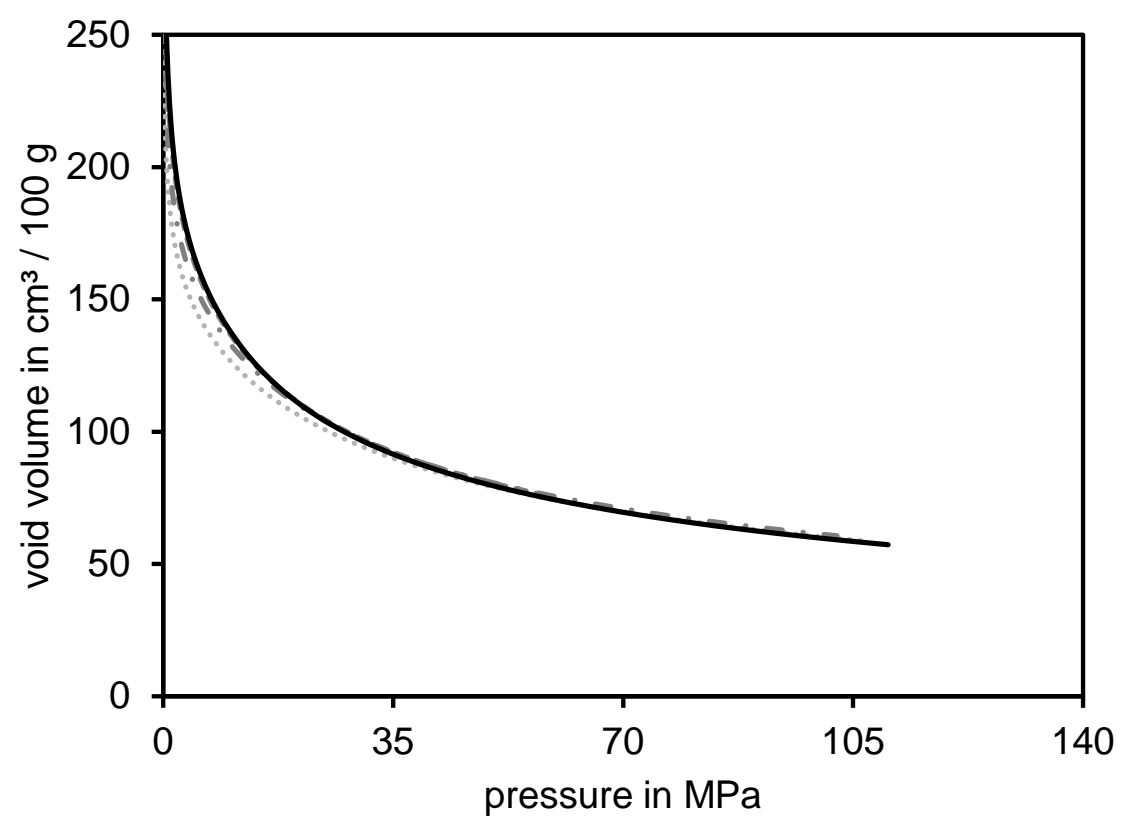

Figure 4.2.7: Void volume measurements of four different types of silica 157MP207 (--); 169P202 (- - -); 104GR175 (‥) and 158GR190 (- - -)

The curves only differ during the compression phase up to $50 \mathrm{MPa}$ and slightly in the maximum value at $125 \mathrm{MPa}$. Due to all the above mentioned facts, it is not possible to evaluate the residual void volume as well as the void-volume values at mean pressure 50,75 and $100 \mathrm{MPa}$ (ASTM standard request [7]) of silica in accordance with the CB void volume method.

\section{Sample preparation}

The best sample preparation was evaluated by investigating three different possible influencing parameter: The influence of the free water content, the influence of the drying time and the effect of grain fractions of granulated silica on the final result.

\section{Moisture/water content (free water) inside the material}

To investigate possible influences on the final result due to different moisture contents all four samples were pre-dried for $2 \mathrm{~h}$ at $105^{\circ} \mathrm{C}$ inside an oven and cooled down to RT in a desiccator for 30 min before being tested in the next step. The time and temperature setting for drying was chosen in accordance with ISO 787-2 [12]. Fig. 4.2.8 shows the results of the four different types of silica with and without a pre-drying step. Each silica was measured three times within both conditions and the void volume values at 5 and $15 \mathrm{MPa}$ pressure were compared. 


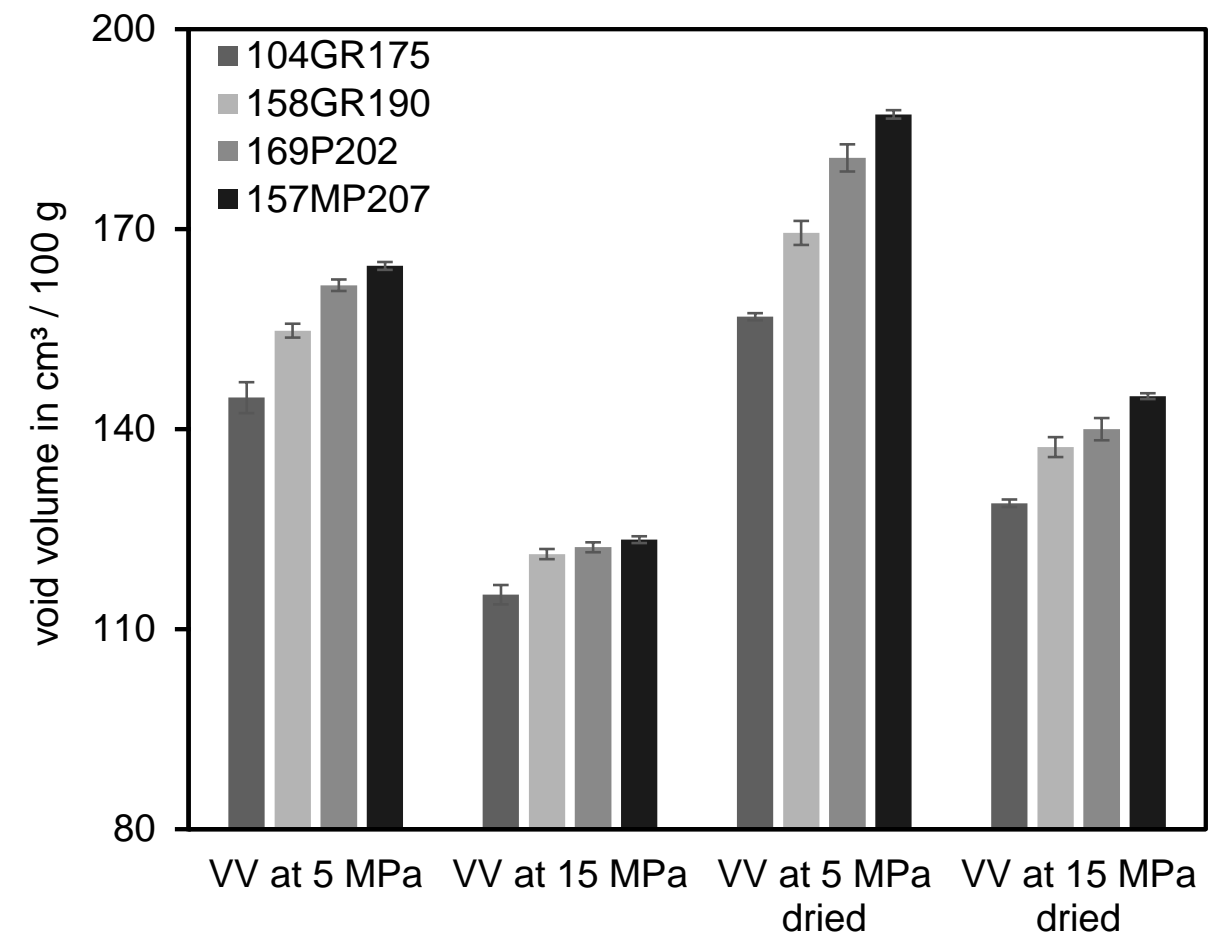

Figure 4.2.8: Void volume measurements of four different types of silica at 5 and $15 \mathrm{MPa}$ in their original state as well as dried for $2 \mathrm{~h}$ at $105^{\circ} \mathrm{C}$

In general, it can be said that the dried samples exhibit higher void volume values at comparable pressure values. This can be explained as following: silica in average contains 4 - 7 wt.\% [13] of free water which can be released during the drying step. $2.0 \mathrm{~g}$ of a dried silica sample therefore contains in total more structured material respectively silica including a certain amount of voids and less non-structured water than $2.0 \mathrm{~g}$ of a non-dried sample. Additionally, the density of the samples differ slightly due to the fact that free water having a density $\rho_{w}$ of $1.0 \mathrm{~g} / \mathrm{cm}^{3}$ within the material and was replaced by silica with a density $\rho_{s}$ of $2.0 \mathrm{~g} / \mathrm{cm}^{3}$. This affects the calculation of the void volume during the measurement in accordance with equations 4.2.1 - 4.2.3.

With increasing pressure it is more difficult to distinguish between different types of silica. Moreover, the dried samples can be differed more easily at low pressure values than the undried ones. The moisture content of one type of silica can also slightly differ from one batch to the other which can affect the accuracy of the final results. Therefore, a pre-drying step was conducted for every following experiment.

Due to these results, the following settings were chosen as the basis for all experiments:

- $\quad$ Sample weight: $2.00 \mathrm{~g}$

- compression: pressure rate of $2 \mathrm{MPa} / \mathrm{s}$ up to a target pressure of $125 \mathrm{MPa}$

- $\quad$ decompression: pressure decreased with a constant rate of $2 \mathrm{MPa} / \mathrm{s}$ to atmospheric pressure)

- $\quad$ pre-drying step: $2 \mathrm{~h}$ at $105^{\circ} \mathrm{C}$ inside an oven

- evaluation: $\quad \mathrm{VV}$ value at $5 \mathrm{MPa}$ mean pressure during the compression phase

\section{Drying time}

As shown beforehand, all silica samples have to be pre-dried before testing. In order to confirm that all free water is released after $2 \mathrm{~h}$ at $105^{\circ} \mathrm{C}$ as assumed $[7,12]$ two different types of silica (195P228 and 196GR210) with relatively high specific surface areas and initial structures were dried for different time periods $(0 \mathrm{~h} / 0.5 \mathrm{~h} / 1 \mathrm{~h} / 2 \mathrm{~h} / 24 \mathrm{~h})$. The test sequence was performed 
as described in the default setting. Each experiment was repeated three times and the standard deviation was determined as depicted in Fig. 4.2.9.

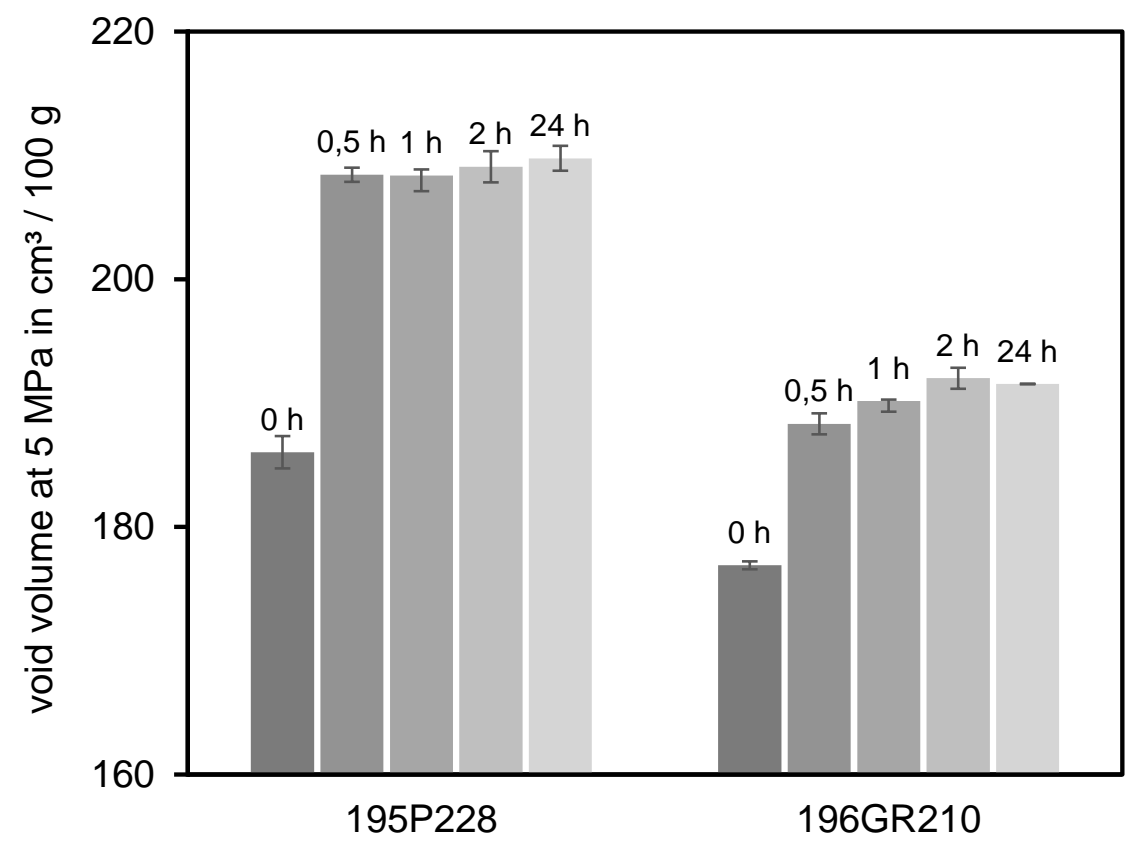

Figure 4.2.9: The effect of drying time at $105^{\circ} \mathrm{C}$ on two types of silica (powder and granule) with relatively high specific surface areas and high initial structures

As shown a drying time of at least $1 \mathrm{~h}$ is needed to achieve a stable condition. In case of granules even a slight difference between 1 and $2 \mathrm{~h}$ can be seen. The dosage form seems to have an influence as well due to the fact that the powder material reaches a constant value already after $30 \mathrm{~min}$. One reason for this might be that it is more difficult to remove free water which is trapped inside the compacted granules having several $\mathrm{mm}$ in diameter compared to an easier release from an open surface in case of a fluffy powder. The investigations confirm that a drying time of $2 \mathrm{~h}$ at $105^{\circ} \mathrm{C}$ is suitable for the sample preparation. Therefore, this procedure was implemented as a standard and used for all following experiments.

\section{Grain fractions and finest of granules}

Silica granules usually have a diameter of a few $\mathrm{mm}$. This type of dosage form can additionally contain a certain amount of fines. Fines is defined as the dusty residual which can be formed during the granulation and transportation of silica granules e.g. due to abrasion between grains. In this way, the size of granules is reduced and a mixture of granules and fines is obtained. To check if this by-product influences the void volume measurement different grain fractions of one type of silica (158GR190) was selected. These fractions were separated by means of sieves with different mesh sizes. The reference sample represents the original sample including all fractions.

Up to now all void volume values were calculated with a silica density of $1.9 \mathrm{~g} / \mathrm{cm}^{3}$ in accordance with the factory specification of the machine supplier. For every following experiments the density of silica is adjusted to $2.0 \mathrm{~g} / \mathrm{cm}^{3}$ [13].

Fig. 4.2.10 depicts the $\mathrm{VV}$ results of the different grain fractions at 5 and $15 \mathrm{MPa}$. It can be seen that the finer the grain size, the higher the void volume at $5 \mathrm{MPa}$. Therefore, it is concluded that the size and as a consequence the dosage form does influence the void volume measurement in the area where the results are evaluated ( $5 \mathrm{MPa})$. The reference and the grain fraction bigger than $300 \mu \mathrm{m}$ show an equal value. One reason for this can be that the total amount of fines in the reference sample is less than $5 \mathrm{wt} .-\%$ and therefore both samples are almost comparable. 


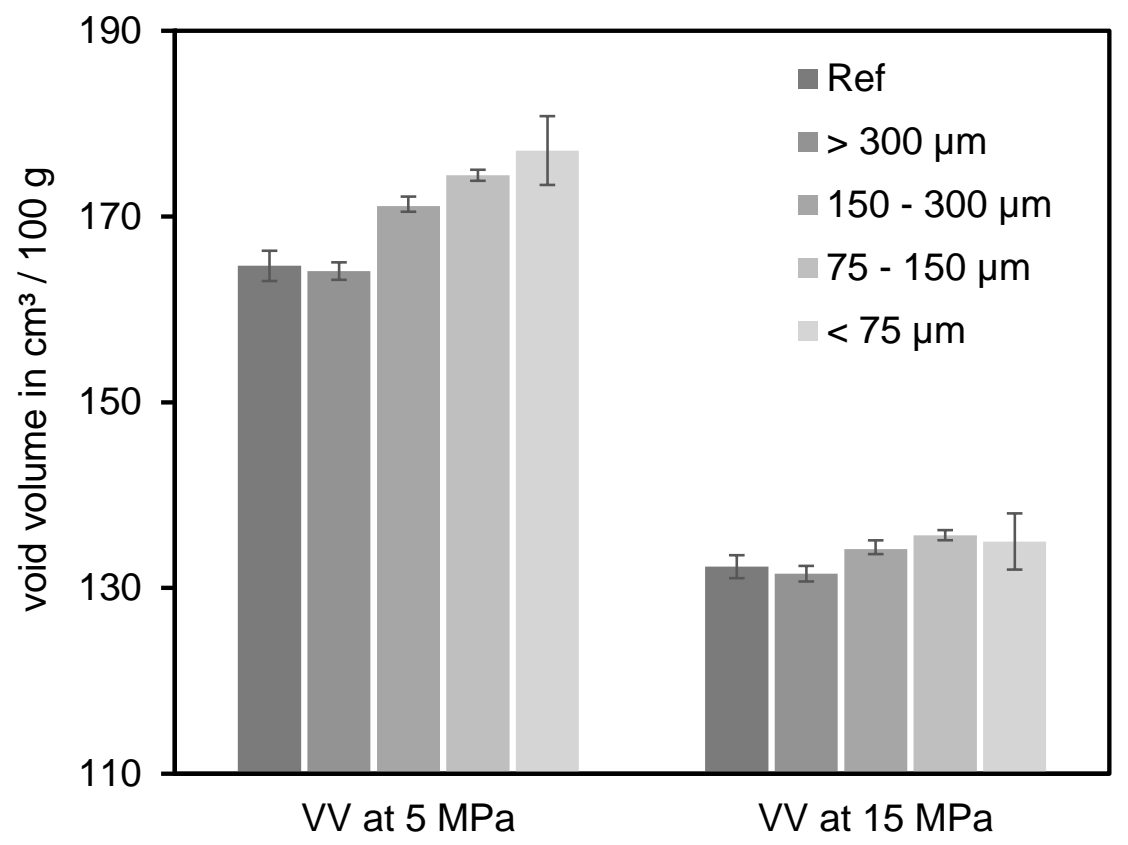

Figure 4.2.10: The effect of different grain sizes of one types of silica on the $V V$ value at $5 \mathrm{MPa}$

The effect of the grain size only can be seen at the low pressure values. Hardly any differences can be recognized at $15 \mathrm{MPa}$ and all samples result in the same end value at $125 \mathrm{MPa}$. Since the amount of fines inside a granule sample can differ from batch to batch it was decided to prepare a size fraction of $>500 \mu \mathrm{m}$ by means of a sieve (mesh width of $0.5 \mathrm{~mm}$ ) in order to use a specific sieve fraction without fines for all granules used in upcoming investigations.

\section{Parameter variations}

Basically, two parameters can be varied for performing a measurement: The sample weight and the measuring procedure. The latter can be subdivided in a path controlled and pressure controlled sequence. Following settings were chosen as reference:

- $\quad$ Sample weight: $2.00 \mathrm{~g}$

- compression: pressure rate of $2 \mathrm{MPa} / \mathrm{s}$ up to a target pressure of $125 \mathrm{MPa}$

- $\quad$ decompression: pressure decreased with a constant rate of $2 \mathrm{MPa} / \mathrm{s}$ to atmospheric pressure)

- $\quad$ pre-drying step: $2 \mathrm{~h}$ at $105^{\circ} \mathrm{C}$ inside an oven

- $\quad$ evaluation: $\quad \mathrm{V}$ value at $5 \mathrm{MPa}$ mean pressure during compression phase

\section{Variation of the weight}

The weight of the sample were varied in four different settings: $0.5 \mathrm{~g} / 2 \mathrm{~g}$ (ref) / $4 \mathrm{~g} / 8 \mathrm{~g}$. Two types of silica were chosen, a powder (169P202) and a granule (158GR190) and each test was repeated three times (Fig. 4.2.11). 


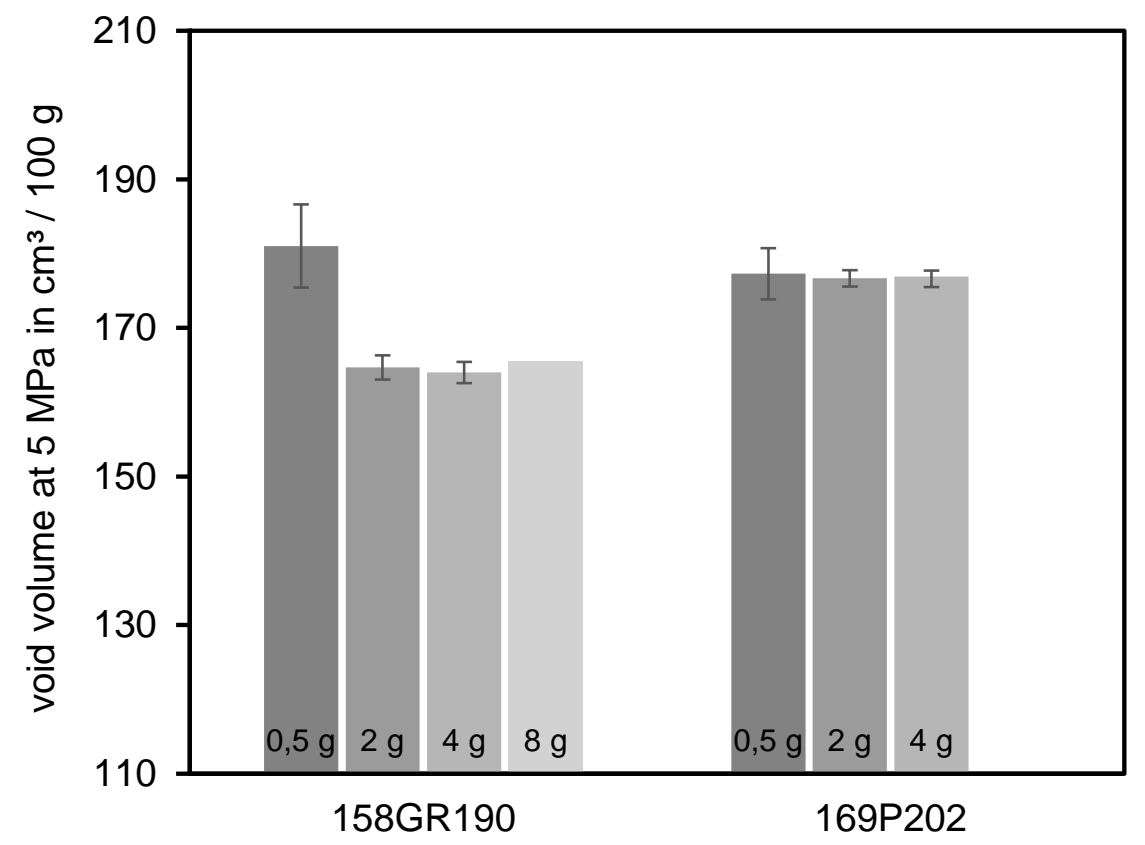

Figure 4.2.11: The effect of the sample weight of two types of silica on the $V V$ value at $5 \mathrm{MPa}$

First of all it can be seen that with a weight of $0.5 \mathrm{~g}$ the standard deviation for the powder and the granules is higher than for the other settings. This results in a poorer repeatability of the measurement and is therefore not recommended to use. Additionally, the $\mathrm{VV}$ value at $5 \mathrm{MPa}$ of the granules differ from all other settings which are on a similar level. The maximum loading of the sample is limited by the size of the measurement chamber. Hence, it was not possible to fill in $8 \mathrm{~g}$ of silica powder. Using $8 \mathrm{~g}$ of granules lead to an increase in the measurement time which is not desired as well. Furthermore, the higher loading leads to a stronger abrasion of the measurement chamber which reduces its lifetime. Hardly any differences can be recognized between the use of 2 and $4 \mathrm{~g}$. The VV values at $5 \mathrm{MPa}$ as well as the standard deviation are on a similar level. As a result, it is concluded that it is more suitable to use the lowest possible amount of silica which corresponds to $2 \mathrm{~g}$ as a fix value. In this way, the measurement time as well as the abrasion of the chamber can be slightly reduced.

\section{Variation of the piston speed: pressure-controlled}

The piston speed during the compression and decompression cycle of a measurement is usually controlled by a fixed pressure-rate of $2 \mathrm{MPa} / \mathrm{s}$. Additionally, two settings were chosen to investigate the influence of a faster (4 MPa / s) and a slower (0.5 MPa / s) speed. Again, two types of silica were chosen, a powder (169P202) and a granule (158GR190) and each measurement was performed three times. Fig. 4.2.12 depicts the results of the $\mathrm{VV}$ values at $5 \mathrm{MPa}$ mean pressure during the compression phase. 


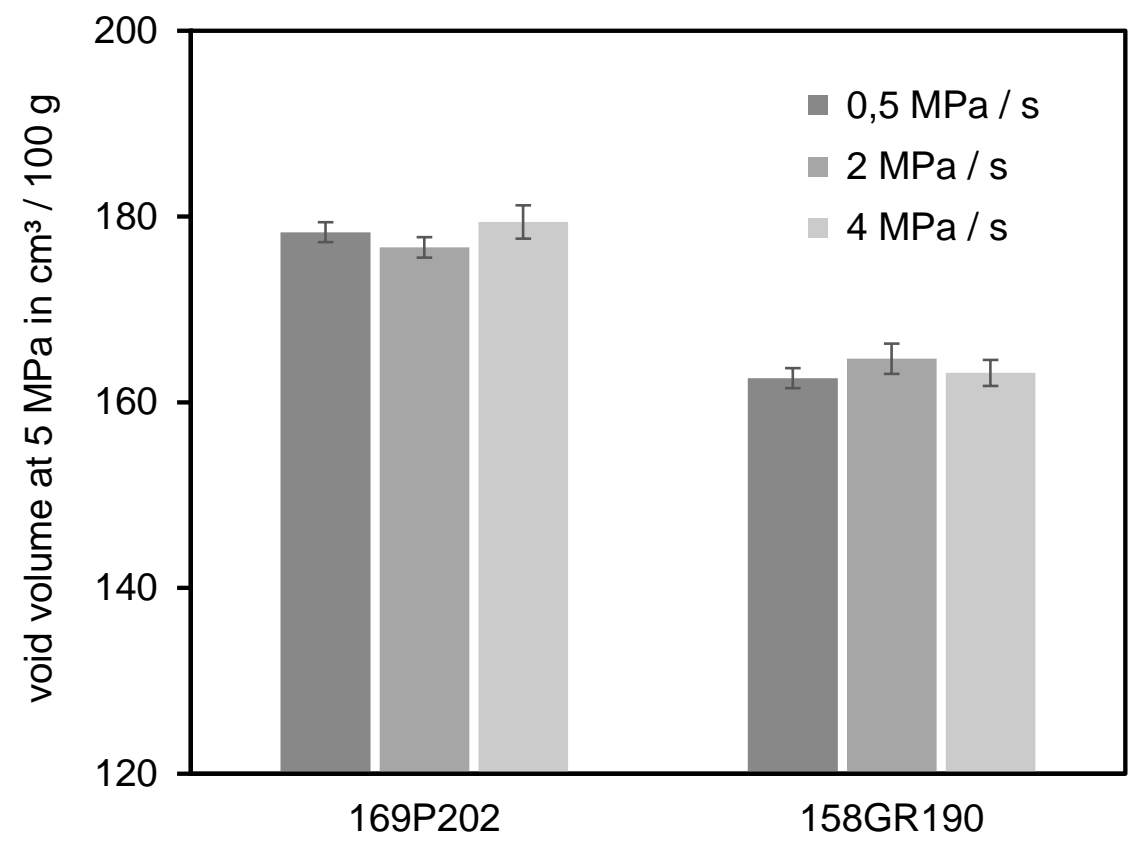

Figure 4.2.12: The effect of the pressure-rate of the piston on the $V V$ value of two types of silica at $5 \mathrm{MPa}$

As can be seen the effect of the pressure-rate to the final results is comparable low. Measuring with $0.5 \mathrm{MPa} / \mathrm{s}$ takes the longest time and is therefore not preferred. However, this method should be applicable for different types of silica with higher structures as well. Due to a certain risk that fast compression cause higher deviations it was decided to choose $2.0 \mathrm{MPa} / \mathrm{s}$ as a compromise.

\section{Variation of the piston speed: path-controlled}

The piston speed during the compression and decompression cycle of a measurement can also be path-controlled by a fixed pace in $\mathrm{mm} / \mathrm{s}$. Therefore two different speeds were chosen and compared to the default settings (pressure rate: $2 \mathrm{MPa} / \mathrm{s}$ ) for a 169P202 and 158GR190.

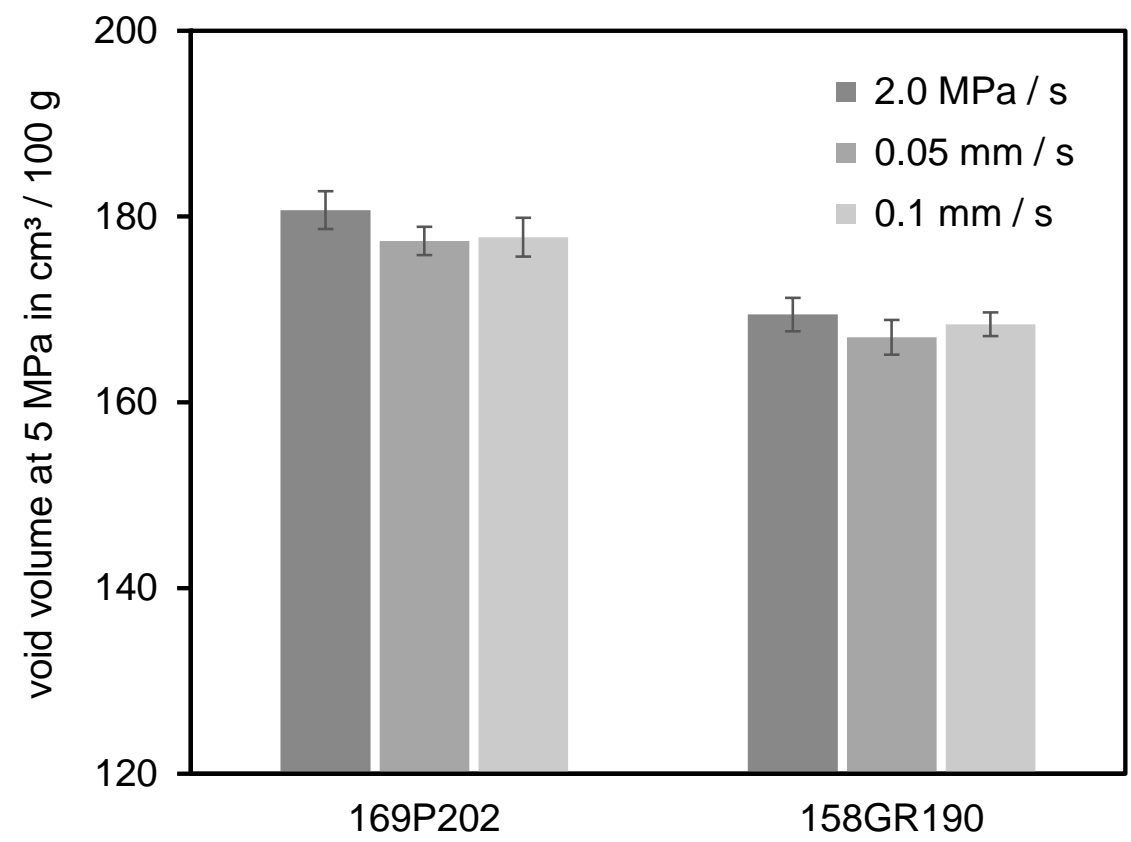

Figure 4.2.13: The effect of the path-rate of the piston on the $V V$ value of two types of silica at $5 \mathrm{MPa}$ in comparison to the default settings 
As shown in Fig. 4.2.13, the pressure-controlled measurement leads to slightly higher $\mathrm{V} V$ values at $5 \mathrm{MPa}$ whereas both path-controlled settings $(0.05 \mathrm{~mm} / \mathrm{s}$ and $0.1 \mathrm{~mm} / \mathrm{s})$ exhibit an equal level. When using a pressure-controlled sequence the piston speed is relatively high at low pressure values due to the fact that the superordinate dosage form is compacted first before the actual structure of the material is compressed. The overall path-controlled measurement is comparable slow at the beginning but the pressure rises non linearly strong during the measurement. This exponential pressure increase is undesirable for the sensitive pressure sensors and should be avoided. Therefore, it was decided to use a pressure-controlled over a path-controlled setting.

\section{Repeatability and Reproducibility}

All investigations on the method development including sample preparation and variation of the process parameters lead to the following final setup:

$\begin{array}{ll}\text { - } & \text { Sample weight: } \\ \text { - } & \text { decompression: } \\ \text { - } & \text { pre-drying step: } \\ \text { - } & \text { evaluation: } \\ \text { granules: }\end{array}$

- $\quad$ Sample weight:

$2.00 \mathrm{~g}$ pressure rate of $2 \mathrm{MPa}$ / s up to a target pressure of $125 \mathrm{MPa}$ pressure decreased with a constant rate of $2 \mathrm{MPa} / \mathrm{s}$ to atmospheric pressure)

$2 \mathrm{~h}$ at $105^{\circ} \mathrm{C}$ inside an oven

$\mathrm{V}$ value at $5 \mathrm{MPa}$ mean pressure during compression phase size fraction of $>500 \mu \mathrm{m}$

To prove the repeatability and reproducibility of this procedure, 16 different silica (powders, granules and micro-pearls) with a wide variety of DOA values as well as other analytical parameters (e.g. BET, CTAB, pH value) were examined. Three different operators tested each silica four times (twelve measurements per type of silica). Based on this, the standard deviation for was determined (Fig. 4.2.14), with a maximum value of $1.33 \%$ for the $173 \mathrm{P} 223$ grade.

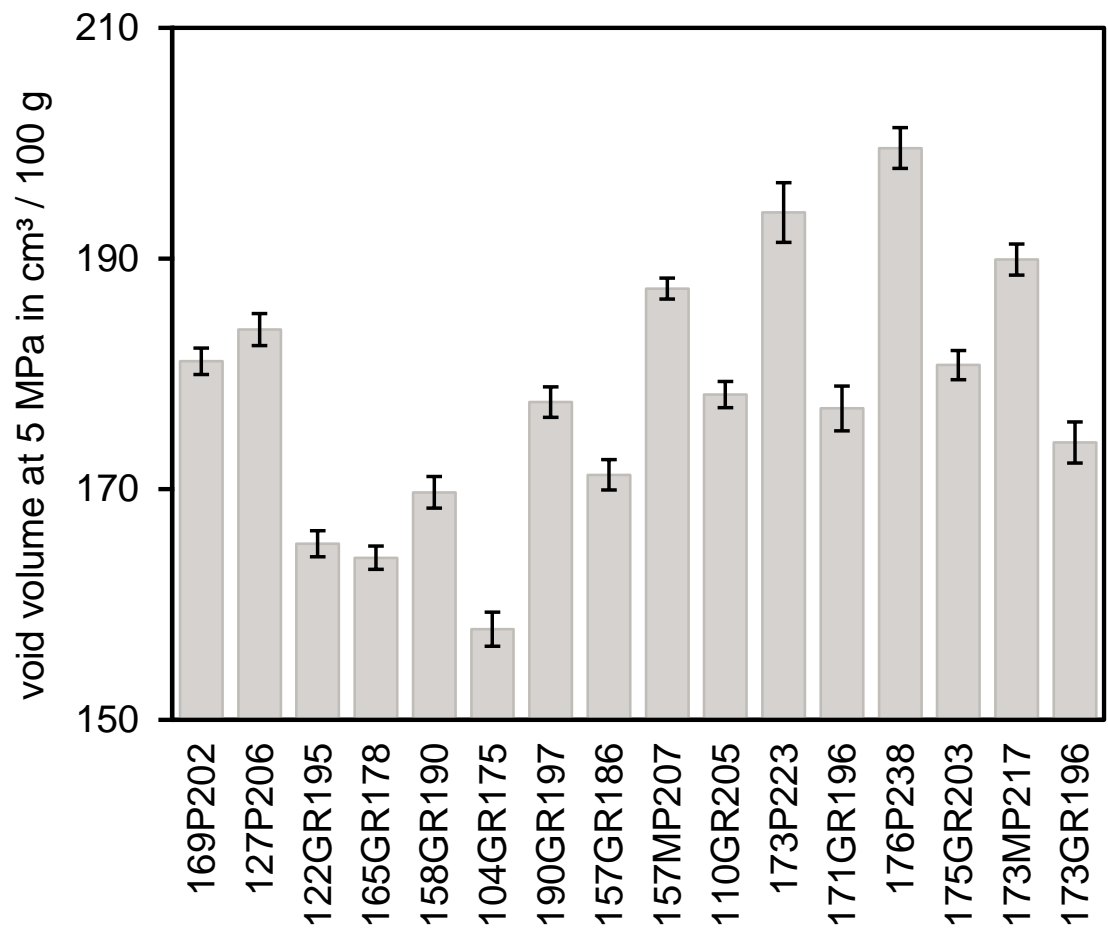

Figure 4.2.14: Reproducibility test of 16 different types of silica conducted by three operators each grade tested 12 times

In accordance with the requirements for a method development it can be concluded that it is possible to achieve reasonable results with this final setup. Different types of silica can be 
distinguished and a sufficient reproducibility is given. The highest standard deviation was for $173 \mathrm{P} 223$ at a level of $1.33 \%$.

\section{Storage effect}

Finally a storage experiment was conducted using the final settings to identify whether the measurement method is dependent on the storage time and on the storage conditions of silica. Therefore, a silica granule (168GR180) was tested directly after the production and stored under the following conditions (Tab. 4.2.2):

Table 4.2.2: Conditions of the storage test for the $\mathrm{VV}$ me
\begin{tabular}{cc} 
Condition & Time \\
\hline Freshly produced & $0 \mathrm{~h}$ \\
$105^{\circ} \mathrm{C}$ (oven) & $24 \mathrm{~h}$ \\
\hline room temperature (RT) & 1 week \\
$50^{\circ} \mathrm{C}$ (oven) & 1 week \\
$-18^{\circ} \mathrm{C}$ (freezer) & 1 week \\
\hline $\mathrm{RT}$ & 1 month \\
$50^{\circ} \mathrm{C}$ (oven) & 1 month \\
$-18^{\circ} \mathrm{C}$ (freezer) & 1 month \\
\hline
\end{tabular}

Each experiment was repeated three times and the standard deviation was determined. Fig. 4.2.15 depicts the results of the $\mathrm{VV}$ values at $5 \mathrm{MPa}$ under different storage times and storage conditions.

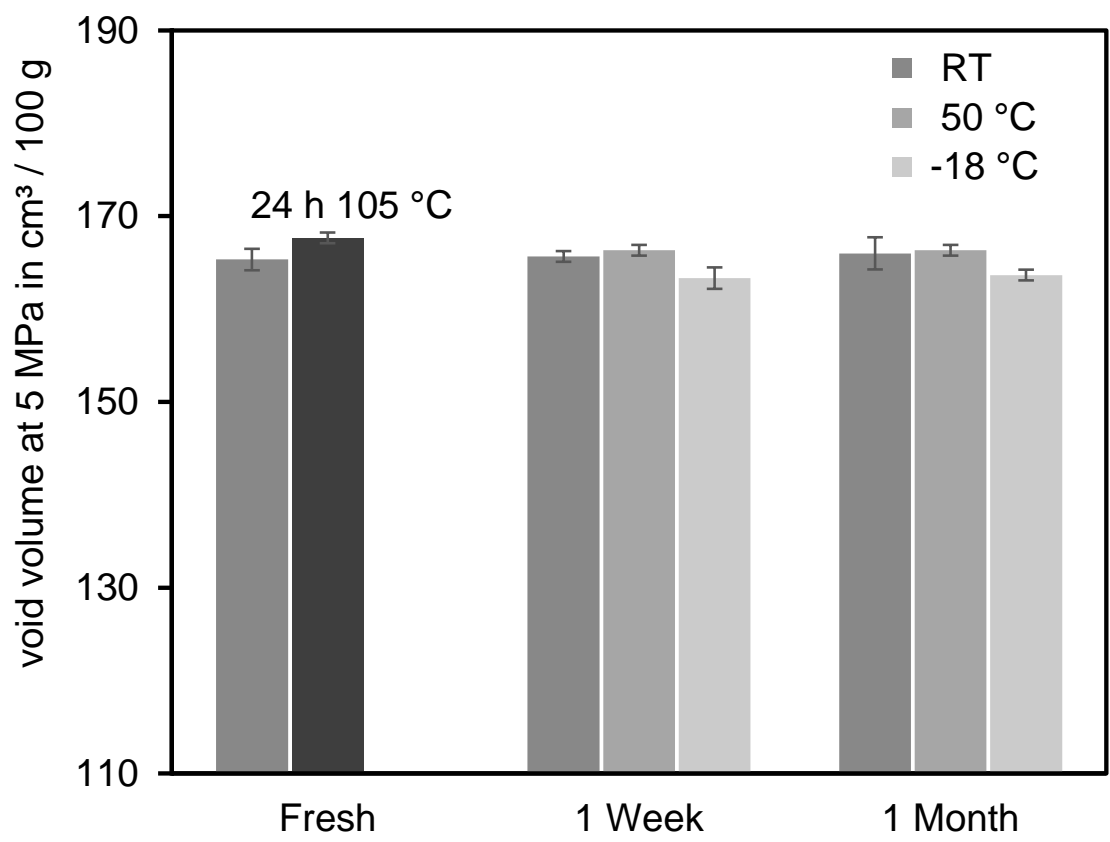

Figure 4.2.15: WV values of one type of silica (168GR180) at $5 \mathrm{MPa}$ being stored under different conditions and periods of time

It can be seen that silica stored inside a freezer leads to lower $\mathrm{V} V$ values in comparison to the source material. One explanation could be that free water trapped inside the silica expands during freezing and thereby softens the granule structure. Therefore, less pressure is needed to break the primary form of the granules and the material can be compressed more easily. As a consequence, the calculated VV at $5 \mathrm{MPa}$ is lower. 
Storing the silica inside an oven at $105^{\circ} \mathrm{C}$ for $24 \mathrm{~h}$ affects the final results as well. The increase in the $\mathrm{VV}$ value after the longer drying time can be explained by the release of additional water trapped inside the granules. This water needs a longer period of time to be released from the material and provide additional free voids. This was already observed during the investigations of the "drying time". However, $2 \mathrm{~h}$ seemed to be sufficient to achieve a constant moisture content.

Silica is usually stored at room temperature (RT) and the investigations confirm that there is no effect on the final result even after 1 month. Each silica sample used in this storage test was investigated in accordance with the final setup, which includes a pre-drying step at $105^{\circ} \mathrm{C}$ for $2 \mathrm{~h}$. Therefore, it seems to be most likely that possible effects due to storage can be overcome by the sample preparation itself.

\subsubsection{Summary and Conclusion \\ Comparison of the DOA and void volume method}

One motivation and aim to investigate the void volume structure measurement was to find an alternative method to determine the initial structure of silica. It was especially desired to overcome the inconveniences to work with oil, to have a relatively time-consuming measurement and a long cleaning step while performing the DOA measurement. Therefore 28 various types of silica with different dosage forms (granules, powders and micro pearls) were selected and the DOA numbers (moisture corrected) [2] and the newly developed void volume values at 5 MPa mean pressure (compression curve) were determined. Fig. 4.2.16 shows the correlation of both measurement systems.

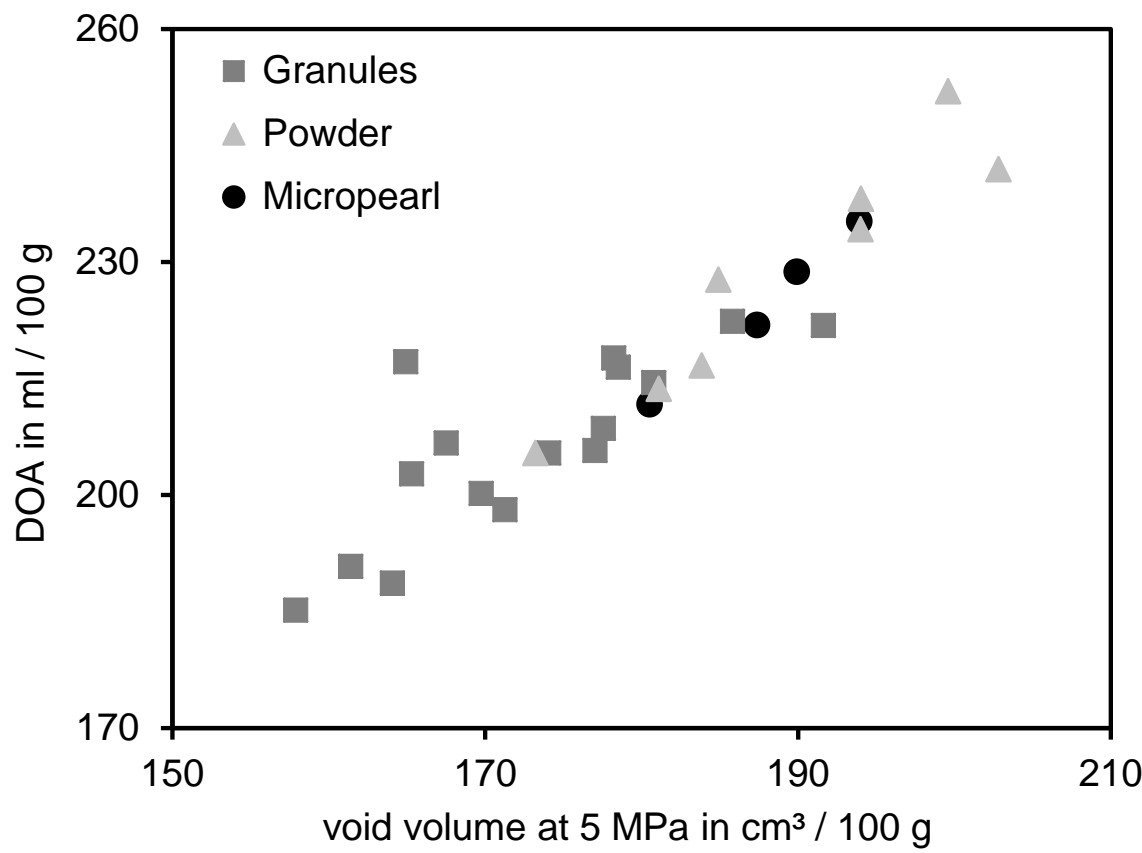

Figure 4.2.16: Correlation between void volume at 5 MPa and DOA measurement

The correlation coefficient of the DOA numbers and void volume values at $5 \mathrm{MPa}$ is 0.92 which indicates that both systems measure the identical analytical parameter. A slight tendency is apparent that powders in general seem to have higher values in comparison to granules. Therefore, it is questionable if both methods are fully independent of the dosage form. By comparing both methods the void volume measurement shows clear benefits over the DOA measurement mainly with regard to the measurement time (Tab. 4.2.3). Additionally, the use of oil can be avoided for this measurement process as well as a long undesirable cleaning step of approximately $5 \mathrm{~min}$. The void volume structure tester can be cleaned by the use of a cleaning brush within $1 \mathrm{~min}$. The absence of additionally required chemicals like oil leads to a very 
clean method without the risk of any health issues. The evaluation of the void volume measurement curves show a tendency to be more precise than the DOA curve evaluation [14] and shows a high repeatability. For the DOA method the moisture content has to be considered whereas for the void volume measurement the silica samples have to be pre-dried. However, this pre-drying step can be performed for several samples at once. A further long-term study is required to determine possible influences due to the degradation of the piston and cylinder.

Table 4.2.3: Comparison of the DOA and void volume method DOA Void Volume

\begin{tabular}{ll}
\hline time ca. $30 \mathrm{~min}$ & time ca. $5 \mathrm{~min}$ \\
cleaning time ca. $5 \mathrm{~min}$ & cleaning time ca. $1 \mathrm{~min}$ \\
working with oil & clean measurement (without oil) \\
fluctuation of results & high repeatability of results \\
moisture content has to be considered & samples have to be pre-dried \\
degradation of chamber and rotors & degradation of piston and cylinder? \\
\hline
\end{tabular}

\section{Summary}

This chapter presents a new measurement system to determine the initial structure of silica. The void volume method is a favorable alternative to the common DOA measurement due to the fact that it performs faster and avoids the use of oil. The measured curves demonstrate that the residual void volume of silica cannot be evaluated in the same way as for carbon black. However, it is possible to evaluate and distinguish silica curves by considering void volume values at specific pressures ( 1 to $50 \mathrm{MPa}$ ) during the compression phase. The measurements show a proper repeatability, a good differentiation between different types of silica and is independent of storage effects. Hence, the new method meets all requirements in accordance with the general basics for method development described in chapter 4.1. The highest impact on the final results occur due to different moisture contents. Therefore, it is recommended to dry all samples before testing and to use a scale with at least two decimal places.

One additional approach could be to use a path-controlled measurement instead. Due to the fact that all types of silica reach the same end value the measurement could be stopped at e.g. $50 \mathrm{MPa}$ to avoid a non-linearly pressure rise. At smaller void volume values, the increase in pressure is lower in comparison to the pressure-controlled procedure and therefore more precise.

\subsubsection{References}

[1] W. M. Hess et al., The influence of Carbon Black, mixing, and compounding variables on dispersion, Rubber Chem. Technol. 57 (1984) 959-1000

[2] ISO 19246:2016 - Rubber Compounding Ingredients - Silica - Oil absorption of precipitated silica

[3] B. Rodgers, Rubber Compounding - Chemistry and Applications, CRC Press, Boca Raton - London - New York (2016)

[4] ASTM D 2414:2016 - Standard Test Method for Carbon Black - Oil Absorption Number (OAN)

[5] Internal Information, Evonik Resource Efficiency GmbH

[6] ASTM D3493:2016 - Standard Test Method for Carbon Black - Oil Absorption Number of Compressed Sample (COAN)

[7] ASTM D7854:2016 - Standard Test Method for Carbon Black-Void Volume at Mean Pressure

[8] P. Hirtt; Y. Leiner, HITEC Luxembourg S.A., US 8464592 B2

[9] Internal Information, HITEC Luxembourg S.A.

[10] M.-J. Wang; S. Wolff, Surface Energy of Carbon Black, Carbon Black, Chapter 6 (Ed. J.B. Donnet), Marcel Dekker Inc., New York (1993) 
[11] ASTM D6556:2016 - Standard Test Method for Carbon Black - Total and External Surface Area by Nitrogen Adsorption

[12] ISO 787-2:1981 - General methods of test for pigments and extenders - Part 2: Determination of matter volatile at 105 degrees $C$

[13] R. K. Iler, The Chemistry of Silica, John Wiley \& Sons, New York - Chichester - Brisbane - Toronto (1978)

[14] T. Bahlke, Measurement system analysis according to VA IM-SI-QM 0001, internal report from Evonik Resource Efficiency, (22.9.2015) 


\section{Chapter 4.3 - Method Development 2: Sedimentation Analysis}

The dispersion process of silica throughout the rubber matrix occurs during the mixing process. To evaluate the dispersibility of silica by means of an analytical method an attempt was made to transfer the dispersion process into the laboratory. Therefore, a solution of silica and demineralized water was treated with ultrasound in order to simulate the dispersion process of silica in rubber by this treatment. Subsequently, the particle size distribution was measured by the use of the sedimentation analyses. Thereby, particles inside the suspension settling by means of gravity, are detected by an X-ray beam which was partly absorbed by the silica. Stokes' law was used to calculate the particles diameter. The lower the amount of residual big particles, the easier it should be to break down clusters respectively disperse the silica.

The sedimentation analyses is dependent on the sample preparation, used concentration of silica in water and the X-ray intensity. Additionally, a dependency due to storage was determined which has to be taken into consideration when measuring silica samples. The strongest influences occur due to the use of ultrasound as a sample preparation method. Especially the wear of the sonotrode has to be monitored in order to avoid deviations of results over a period of time.

This sedimentation method was successfully implemented to use it for characterizing silica samples. A suitable reproducibility was achieved. It was shown that it is possible to distinguish between different types of silica.

\subsubsection{Introduction}

The dispersion mechanism of silica is a complex process which is widely discussed in literature and various approaches are made to get a deeper inside and understanding as described in chapter 2.4. To predict the dispersibility of silica by means of an analytical method it is therefore necessary to simplify this process by having a look into the basic requirements for dispersion itself. To simulate the dispersion process, silica has to be reduced in its size. Doing this, two main factors have to be taken into consideration. On the one hand, an energy input is needed to break down clusters to a smaller scale. In practice, this corresponds to e.g. the internal mixer which applies shear forces by means of its rotors. On the other hand, a matrix is needed, in which the material is distributed and which transfers the energy to the silica itself. This refers to the rubber itself.

Following this idea, an approach was made to transfer the dispersion process into the laboratory by means of a new analytical method. Therefore, different sample preparation methods to apply defined energy to the material were conducted. Subsequently, the particle size distribution of the samples were measured. In this way, information about the easiness of cluster breakage can be obtained. The smaller the particles and the less big clusters remain after energy input, the easier it should be to reduce the particle size respectively to disperse the silica. To measure the particle size distribution the sedimentation method [1] was taken into account. Silica particles settle inside a solution (matrix) by means of gravity. The heavier the particles, the faster they settle. The size of clusters are detected by an X-ray beam which is partly absorbed by the material.

In the present chapter, silica was investigated by means of this sedimentation method. Different silica samples with a variety of analytical parameters and dosage forms (powders, granules and micro-pearls) were investigated. The measurement curves were evaluated and it was checked if this analysis meets the requirements for a newly developed method in accordance with what was claimed in chapter 4.1. 


\subsubsection{Theoretical principles}

The sedimentation system (SediGraph III Plus by Micromeritics GmbH [2]) consists of a fixed X-ray source and a detector. In between these components a moveable vertical measurement cell is situated, connected to a tube system in which the sample solution can circulate (Fig. 4.3.1).

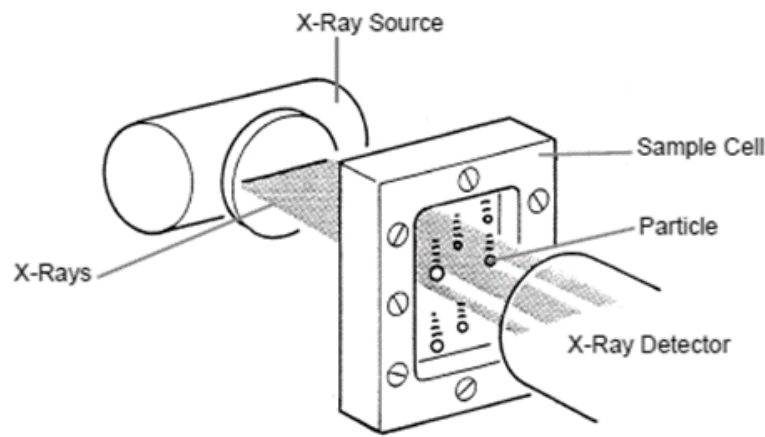

Figure 4.3.1: Schematic view of the sedimentation method including filler sample [2]

During a measurement cycle, silica particles settle inside a water suspension by means of gravity. The heavier the particles, the faster they settle. The absorption of an X-ray beam ( $0.125 \mathrm{~mm}$ wavelength) by the silica clusters is measured. This is based on the Beer-Lambert law of extinction describing the extent to which the intensity of a beam is exponentially reduced by passing through an absorber. It is dependent on the absorption coefficient and the concentration of the suspension and the distance the beam has to pass. This special characteristic results in a benefit over optical light- and laser-based measurement systems where next to the absorption also scattering, refraction and diffraction effects have to be taken into consideration. With known viscosity of the fluid, density of the fluid and density of the silica the spheres' diameters are calculated by the use of Stokes' law. This calculation is based on the assumption that the equilibrium velocity of particles through a defined viscous medium can be related to the diameter of the particles (eq. 4.3.1) [3]:

where:

$$
D_{s}=K * v^{1 / 2}
$$

$$
K=\sqrt{\frac{18 * \eta}{\left(\rho-\rho_{0}\right) * g}}
$$

$D_{s}$ represents the calculated sphere diameter in $\mu \mathrm{m}, K$ is a combination of constants, $\rho$ is the filler's density in $\mathrm{g} / \mathrm{cm}^{3}$ which is for silica $2.0 \mathrm{~g} / \mathrm{cm}^{3}$ [4] and $v$ is the equilibrium sedimentation velocity of the particle in $\mathrm{m} / \mathrm{s}$. The fluid is characterized through its density $\rho_{0}$ in $\mathrm{g} / \mathrm{cm}^{3}$ and viscosity $\eta$ in Pas. The gravity $g$ is given in $\mathrm{m} / \mathrm{s}$. The combination of eq. 4.3 .1 and eq. 4.3.2 results in the Stokes' law equation (eq. 4.3.3):

$$
D_{s}=\sqrt{\frac{18 * v * \eta}{\left(\rho-\rho_{0}\right) * g}}
$$

As a matter of fact, silica particles are irregular shaped and therefore do not meet the requirements for being described by a single linear dimension. Therefore, the term "Stokes' diameter" was introduced. It is defined as the diameter of a perfect sphere (identical material) that settle with the same sedimentation velocity as the actual particle. This assumption is very well accepted in practical use [2]. 
Another requirement for using Stokes' law to calculate particle sizes during sedimentation is to have a laminar flow. Therefore, a low settling velocity and a Reynolds number (eq. 4.3.4) of less than 0.3 is required. The Reynolds number is a dimensionless relationship between the particle diameter and its sedimentation velocity [5].

$$
\boldsymbol{R} \boldsymbol{e}=\frac{\boldsymbol{D} * \boldsymbol{v} * \boldsymbol{\rho}_{\mathbf{0}}}{\boldsymbol{\eta}}<0.3
$$

Assuming that Stokes' law is valid, all particles of an equivalent spherical diameter $D$ settle a specific distance $h$ during the time $t$ as expressed in equation 4.3.5 [3]:

$$
D=K *\left(\frac{h}{t}\right)^{1 / 2}
$$

As a consequence, all particles inside the suspension lager than a diameter $D_{i}$ overcome a distance $h$ within a time $t_{i}$. With the initial uniform concentration of particles $C_{s}$ in $\mathrm{g} / \mathrm{ml}$ and the concentration $C_{i}$ in $\mathrm{g} / \mathrm{ml}$ after the time $t_{i}$ at the distance $h$, the weight percent of particles $P_{i}$ finer than $D_{i}$ can be calculated [3]:

$$
P_{i}=100 * \frac{C_{i}}{C_{s}}
$$

As a result, the particle sizes and distribution of the suspension based on the total mass (\% by volume) is determined by the sedimentation analysis.

In addition to the requirements for the particles and calculation, following aspects have to be taken into consideration as well: The fluid is supposed to have infinite extend which requires a low volume concentration of maximum $1 \% \mathrm{v} / \mathrm{v}$ and no wall effects should occur. It is therefore recommended to have at least a distance of $5 \mathrm{~mm}$ from sidewall to sidewall of the measurement cell [5]. The particle size analysis via sedimentation is limited to particles bigger than $0.1 \mu \mathrm{m}$. The Brownian motion of smaller particles is too high to allow an effective settling by means of gravity unless they have a very high density.

The suspension inside the measurement cell has the highest concentration of particles at the beginning of the measurement and therefore the lowest X-ray intensity is detected. During settling of the particles the concentration decreases and the recorded X-ray intensity increases as depicted in Fig. 4.3.2 [6].

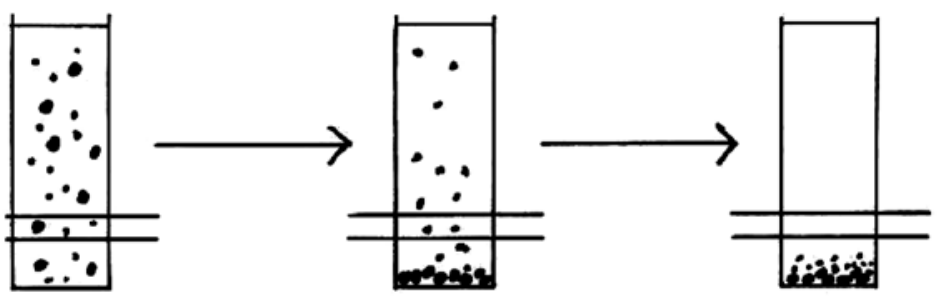

Figure 4.3.2: Scheme of the integral sedimentation method [6]

This principle of measurement is referred to as the integral sedimentation method [7]. It is named due to the fact that the sum or "integral" of all particles smaller than a particular diameter are continuously calculated during the measurement. The particle size distribution is evaluated as shown in Fig. 4.3.3 where the concentration of particles (cumulative mass finer) is plotted against the calculated diameters. 


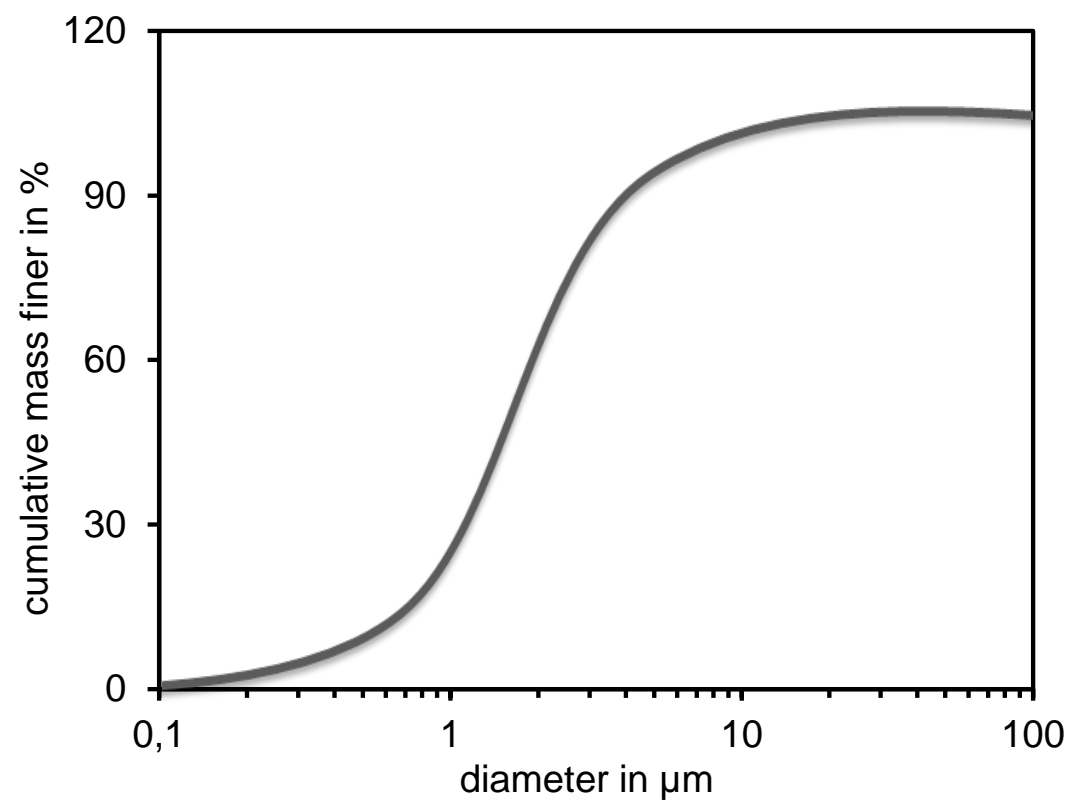

Figure 4.3.3: Typical particle size distribution curve measured by the sedimentation analysis

At the beginning of the measurement, when the concentration of particles is the highest, the cumulative mass finer is at $100 \%$ which implies that no settling took place yet. Assuming all particles have settled and only pure fluid is left inside the measurement cell the cumulative mass finer reaches $0 \%$ which corresponds to the measured baseline intensity. Due to the fact that the heavier the particles, the faster they settle, the biggest clusters are recorded at first. It is possible to detect particle sizes between 300 to $0.1 \mu \mathrm{m}$. However, the measurement time to detect all sizes down to $0.1 \mu \mathrm{m}$ takes about $3 \mathrm{~h}$ [2].

\subsubsection{Method Development}

In order to develop a suitable method to investigate different types of silica the basic principles as described in chapter 4.1 have to be taken into account. Therefore, it is necessary to be aware of the parameters which can be varied, namely the machine settings on the one hand and the sample preparation on the other hand. Following list summarizes the investigations which were conducted:

- Default Settings

- Sample Mass and Concentration

- Sample Preparation

- Speed of measurement (analysis type)

- X-ray Intensity

- Repeatability and Reproducibility

- Storage effect

In order to reduce influences due to impurities and aging of the X-ray beam, the Sedigraph was rinsed each new measurement day and a new baseline was defined. 


\section{Default Settings}

As a starting point for the method development certain machine parameters have to be chosen. Therefore, following general assumptions and settings were made:

$\begin{array}{lll}\text { - } & \text { Analysis Type: } & \text { high speed } \\ \text { - } & \text { Analysis Temperature: } & 35^{\circ} \mathrm{C} \\ \text { - } & \text { Analysis Liquid } & \text { demineralized water } \\ \text { - } & \text { X-Ray Intensity: } & \text { Normal } \\ \text { - } & \text { Density Silica: } & 2.0 \mathrm{~g} / \mathrm{cm}^{3}\end{array}$

Due to the fact that the sedimentation speed of fine particles by means of gravity is relatively low the analysis type "high speed" was selected to reduce the measurement time. During "high speed" the measurement cell slowly moves upwards to detect particles earlier. As a liquid demineralized water was used and the analysis temperature was set to $35^{\circ} \mathrm{C}$. With these parameters the software was able to calculate the precise viscosity of the liquid which is necessary to determine the diameters of the particles more precisely in accordance with eq. 4.3.3. Finally, the X-ray intensity of the beam was set to "normal" and the silica density was assumed to be $2.0 \mathrm{~g} / \mathrm{cm}^{3}$.

\section{Sample Mass and Concentration}

The first parameter to be varied was the concentration of the suspension. On the one hand, the concentration has to be high enough to reach a sufficient X-ray absorption of silica at the beginning of the measurement. On the other hand, if the concentration is too high the suspension absorbs most of the X-rays and hardly any intensity can be detected. It is recommended by the manufacturer to have a reduction of the intensity of minimum $30 \%$ to maximum $70 \%$ due to the presence of silica in the liquid compared to the pure liquid. The result of measuring the residual X-ray intensity of pure liquid is used as the baseline [2]. The measurement system requires $50 \mathrm{ml}$ of suspension in total. Therefore, the amount of liquid has to be kept constant $(50 \mathrm{ml})$ but the amount of silica can be varied.

With regard to the used liquid two influencing parameters have to be taken into account. Firstly, the liquid has to be inert to the measurement device (e.g. tubes and cell) and secondly, the liquid has to inhibit the silica to re-agglomerate. Therefore, the easiest solution is to use demineralized water [8].

One silica granule (215GR210) was rubbed through a $500 \mu \mathrm{m}$ sieve and mixed with $50 \mathrm{ml}$ of water in three different concentrations, 4,5 and $6 \mathrm{~g}$. The size reduction was crucial due to the fact that the granules do not dissolve in water and therefore no suspension could be obtained. Subsequently, the suspensions were measured to evaluate the reduction of the X-ray intensity expressed in kiloCounts per second in comparison to the baseline.

\begin{tabular}{cccc}
$\begin{array}{c}\text { Silica } \\
\text { in } \mathbf{~ g}\end{array}$ & $\begin{array}{c}\text { Table 4.3.1: Influence of the silica concentration on the X-ray absorption } \\
\text { Baseline X-ray counts } \\
\text { in kCnts / s }\end{array}$ & $\begin{array}{c}\text { Suspension X-ray counts } \\
\text { in kCnts / s }\end{array}$ & $\begin{array}{c}\text { Reduction of } \\
\text { baseline in \% }\end{array}$ \\
\hline 4 & 140 & 100 & 28.6 \\
5 & 141 & 92 & 34.3 \\
6 & 140 & 85 & 39.3 \\
\hline
\end{tabular}

As can be seen in Tab. 4.3.1, $4 \mathrm{~g}$ of silica is too low in accordance with the above mentioned requirement of at least $30 \%$ reduction of the baseline. Both, 5 and $6 \mathrm{~g}$ of the sample can be used within the required conditions. However, the handling and mixing of $6 \mathrm{~g}$ of silica with $50 \mathrm{ml}$ of water is more difficult and therefore not preferred for a daily lab routine.

Therefore, $\mathbf{5} \mathbf{g}$ of silica with $\mathbf{5 0} \mathbf{~ m l}$ of demineralized water was chosen as a final setting for all following experiments. 


\section{Sample preparation}

To achieve a suitable sample preparation method, the following approaches were made:

- Measuring without an additional sample preparation step

- Rubbing samples through a sieve with a mesh size of $500 \mu \mathrm{m}$

- Milling the samples by means of a rotor mill (Fritsch) with a mesh size of $80 \mu \mathrm{m}$

- Milling the samples by means of a universal mill (IKA)

- Treating a silica solution by means of ultrasound

First of all, it was tested if it is possible to perform a measurement without any sample preparation, being independently of the dosage form. Powders and micro pearls in general can be solved with water whereas granules keep their shape. Therefore, no suspension could be achieved with granules to be measured. Additionally, the size of granules are partly too big ( $>5 \mathrm{~mm}$ in diameter) to pass the tubes of the measurement system ( $3 \mathrm{~mm}$ in diameter). As a consequence, it was not possible to measure silica without any sample preparation.

A second approach was made to overcome the difficulties of previously mentioned test. All samples thus were rubbed through a $\mathbf{5 0 0} \boldsymbol{\mu m}$ sieve to reduce the initial size. Powders as well as micro pearls have a particle size of less than $500 \mu \mathrm{m}$ and therefore passed the sieve without additional force. In contrast to this, granules were carefully rubbed through the sieve by hand. Solving the particles in water was possible, however, they quickly settled and separated from the fluid. This resulted in difficulties by partially blocking the inner tubes of the measurement system and it was no longer possible to perform the experiments. Consequently, this sample preparation is also not sufficient.

To achieve a more homogeneous suspension which is able to be tested by means of the sedimentation analyses a smaller particle size is needed. Therefore, a rotor mill was taken into consideration.

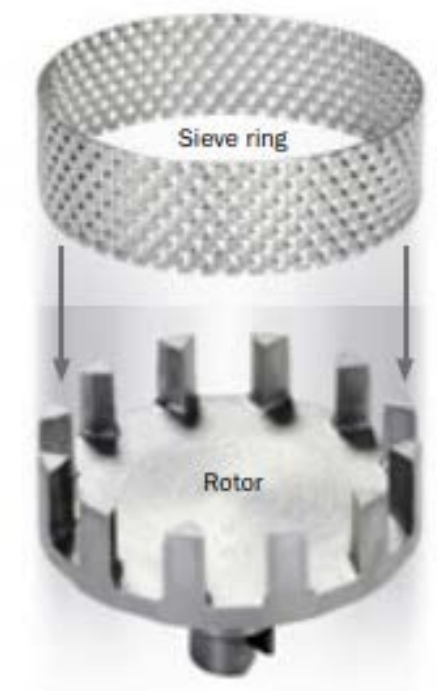

Figure 4.3.4: Principle setup of a Fritsch mill including a rotor with ribs and an outer sieve ring with a defined mesh size [9]

The Pulverisette by FRITSCH GmbH or Fritsch mill (Fig. 4.3.4) consists of a centered rotor including ribs and a sieve ring surrounding it ( $80 \mu \mathrm{m}$ mesh size). The sample is added into the spinning device (20000 rpm) and passes the sieve by centrifugal forces. Thereby, the samples are sheared between the rotor teeth and the sieve and forced to pass it to the outside. 
Three different silica granules (159GR204, 165GR181 and 197GR199) were prepared by the help of the Fritsch mill. Subsequently, $5 \mathrm{~g}$ of each sample was mixed with $50 \mathrm{ml}$ of demineralized water and tested three times in accordance with the default settings. Fig. 4.3.5 depicts the particle size distribution as a function of the calculated diameters.

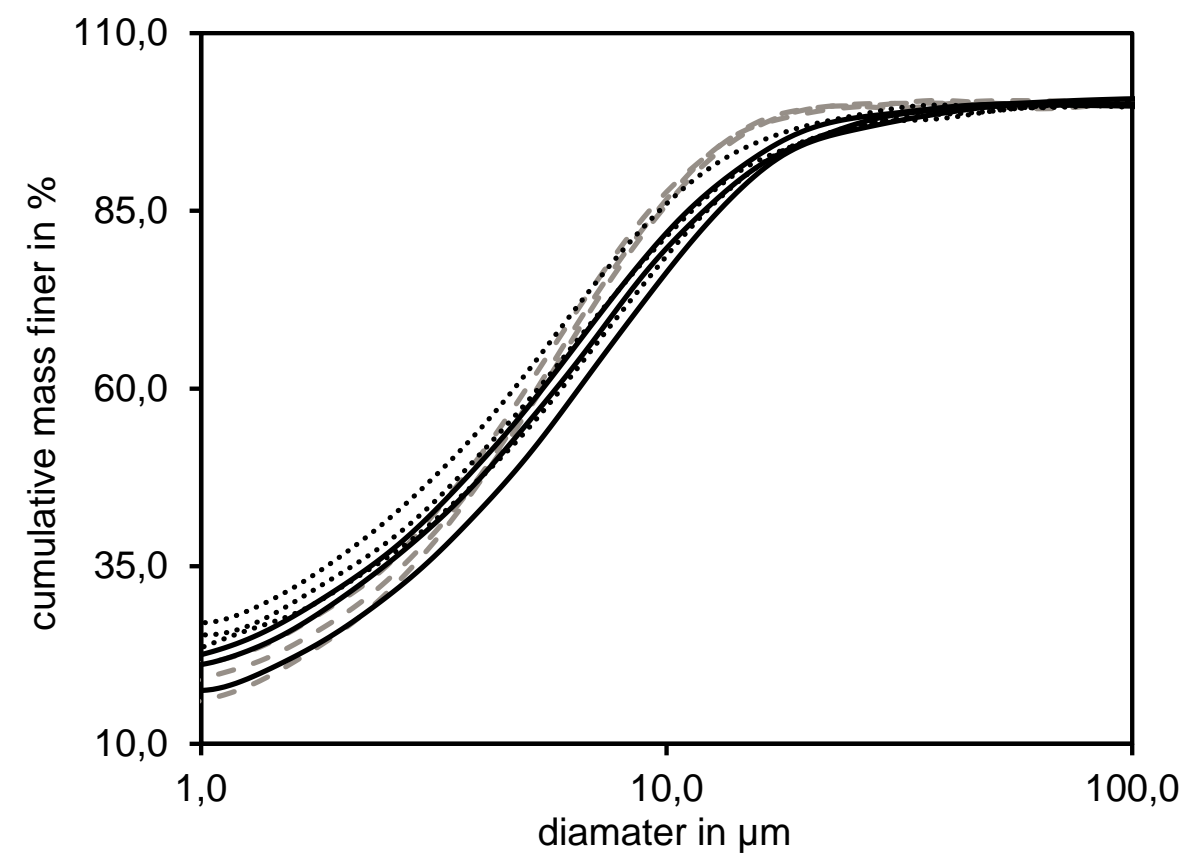

Figure 4.3.5: Sedimentation analyses of three different silica granules 197GR199 (一); 165GR181 (- - ) and 159GR204 (...) after Fritsch mill treatment

It is shown that the biggest particles detected for each sample are in a size range of $50 \mu \mathrm{m}$. However, most particles seem to have a diameter of less than $15 \mu \mathrm{m}$. The repeatability is comparably low and it is hardly possible to differ between different types of silica. Additionally, it was not possible to prepare powders and micro-pearls with the Fritsch mill due to the fact that they were only partly pushed through the sieve and mostly remained inside the middle of the rotor. Another disadvantage of this technique is, that the samples are just sheared shortly before passing the mesh. The particle size distribution therefore strongly varies beneath $80 \mu \mathrm{m}$. Consequently, this technique is not useful as a sample preparation method.

To achieve a more homogeneous particle size reduction a universal mill by IKA-Werke GmbH \& Co. KG was tested as another sample preparation method. The IKA mill consists of a closed chamber including a rotating blade. The silica sample $(10 \mathrm{~g})$ was filled inside this chamber and the blade sheared the material at a speed of $20000 \mathrm{rpm}$. By varying the preparation time the particle size reduction can be controlled. During the procedure, the sample fully remains inside the mixing chamber and, in theory, all particles get permanently sheared throughout the preparation time. It turned out that silica particles randomly got stuck to the chamber wall and hence did not get sheared any further. Additionally, malfunction frequently occurred and the mill had to be maintained. Eventually it was also not possible to prepare powders and micro pearls with this technique because they were already to fine to be sheared sufficiently. Therefore, also this preparation technique is not applicable.

Previous sample preparation trials including different mills showed that it is necessary to reduce the particle sizes over a defined period of time to guarantee a homogeneous energy input to the whole sample. Therefore, a solution of silica and water was treated by ultrasound. As described in chapter 4.1 ultrasound is one of the most sensitive sample preparation methods with various influencing parameters. As a consequence, following tests were performed: 
- Variation of amplitude and time

- Evaluation of results

- Effect of cooling during ultrasonic treatment

- Influence of the position of the sonotrode

- Influence due to inaccurate concentration

\section{Parameter variations of the ultrasonic treatment Ultrasonic Amplitude and Time}

The energy input during the ultrasonic pre-treatment can be controlled by two factors: the amplitude and the sonification time. In order to develop the most suitable sample preparation parameters with on the one hand a high repeatability and on the other hand a good distinguishability, two different silica granules were tested. The 158GR190 is known to be a highly dispersible silica which is expected to show a low amount of bigger clusters even after low energy input. The 165GR178 on the contrary is expected to show a higher amount of bigger clusters even after a higher energy input. Therefore, both types of silica are tested within various conditions of time and amplitude as depicted in Tab. 4.3.2.

Table 4.3.2: Different tested combinations of ultrasonic amplitude and time
\begin{tabular}{cccc}
$\mathbf{1 0 0} \%$ & $\mathbf{7 0} \%$ & $\mathbf{5 0} \%$ & $\mathbf{3 0} \mathbf{0}$ \\
\hline $15 \mathrm{~min}$ & $15 \mathrm{~min}$ & $15 \mathrm{~min}$ & $15 \mathrm{~min}$ \\
$10 \mathrm{~min}$ & $10 \mathrm{~min}$ & $10 \mathrm{~min}$ & $10 \mathrm{~min}$ \\
$5 \mathrm{~min}$ & $5 \mathrm{~min}$ & $5 \mathrm{~min}$ & $5 \mathrm{~min}$ \\
$2 \mathrm{~min}$ & $2 \mathrm{~min}$ & $2 \mathrm{~min}$ & $2 \mathrm{~min}$ \\
\hline
\end{tabular}

The silica sample were prepared as follows: $5 \mathrm{~g}$ of silica were mixed with $50 \mathrm{ml}$ of demineralized water. An ultrasonic device (Branson Digital Sonifier 450D incl. a flat $1 / 2$ " tip by BRANSON Ultrasonic Coop.) was used to treat the suspension while being cooled in a water bath (beaker halfway immersed) at $5{ }^{\circ} \mathrm{C}$. As already mentioned in chapter 4.3.2, it is possible to measure the particle size distribution from 300 to $0.1 \mu \mathrm{m}$ but it would take about $3 \mathrm{~h}$ to record the smallest sizes. Due to the fact that the final results are correlated to the in-rubber macro-dispersion measurements it was decided to "go down" to $1 \mu \mathrm{m}$ and subsequently stop the measurement. In this way the analyzing time was reduced to 8 min which makes the method applicable for a daily lab routine.

As a first result it turned out that silica granules cannot be directly prepared via ultrasound inside a suspension. During the treatment bigger fragments of the granules got stuck to the sidewall of the sonotrode and hence did not take part in the size reduction. This phenomenon occurs randomly and to an irregular extent which results in an insufficient treatment. Therefore, all silica granules were rubbed through a $500 \mu \mathrm{m}$ sieve beforehand. With this additional step it is also possible to prepare granules via the ultrasonic treatment.

\section{$30 \%$ of amplitude}

An amplitude of $30 \%$ led to insufficient energy input independently of the treatment time. Particles were settled during the sonification and could hardly be reduced in size. Therefore, the suspension partially blocked the inner tubes of the measurement system similarly to the samples only being rubbed through a $500 \mu \mathrm{m}$ sieve. Hence, the amplitude has to be increased.

\section{$50 \%$ of amplitude}

Fig. 4.3.6 depicts the results of the two types of silica treated with $50 \%$ of amplitude for 2, 5, 10 and 15 min with ultrasound. The concentration of particles (cumulative mass finer) is plotted against the calculated diameters. 

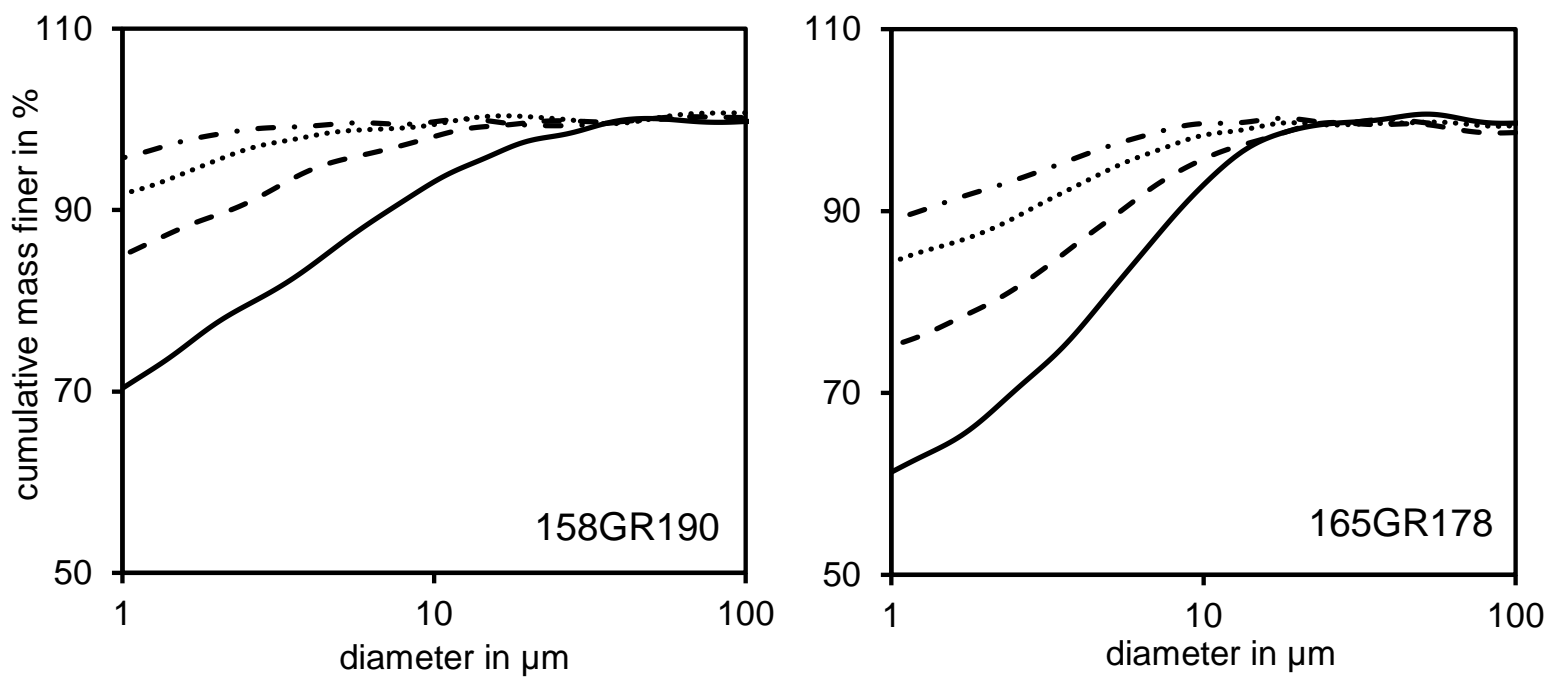

Figure 4.3.6: Analyses of 158GR190 and 165GR178 at $50 \%$ amplitude with 2 min (-), $5 \mathrm{~min}(-$ - $),_{10} \mathrm{~min}(. .$.$) and 15 \mathrm{~min}\left(-_{-}-\right)$ultrasonic pre-treatment

For both types of silica the ultrasonic treatment time strongly affects the particle size reduction. The longer the suspensions are treated, the less particles e.g. bigger than $1 \mu \mathrm{m}$ can be identified. It can be noticed that 165GR178 always shows a higher amount of larger clusters at identical times and diameters in comparison to 158GR190. Additionally, even after short treatment times (2 min) about 70 \% (158GR190) respectively $60 \%$ (165GR178) of all particles inside the suspensions are smaller than $1 \mu \mathrm{m}$.

\section{$70 \%$ of amplitude}

Fig. 4.3.7 depicts the results of the before mentioned two types of silica treated with $70 \%$ of amplitude for 2, 5, 10 and 15 min with ultrasound. The concentration of particles (cumulative mass finer) is plotted against the calculated diameters.
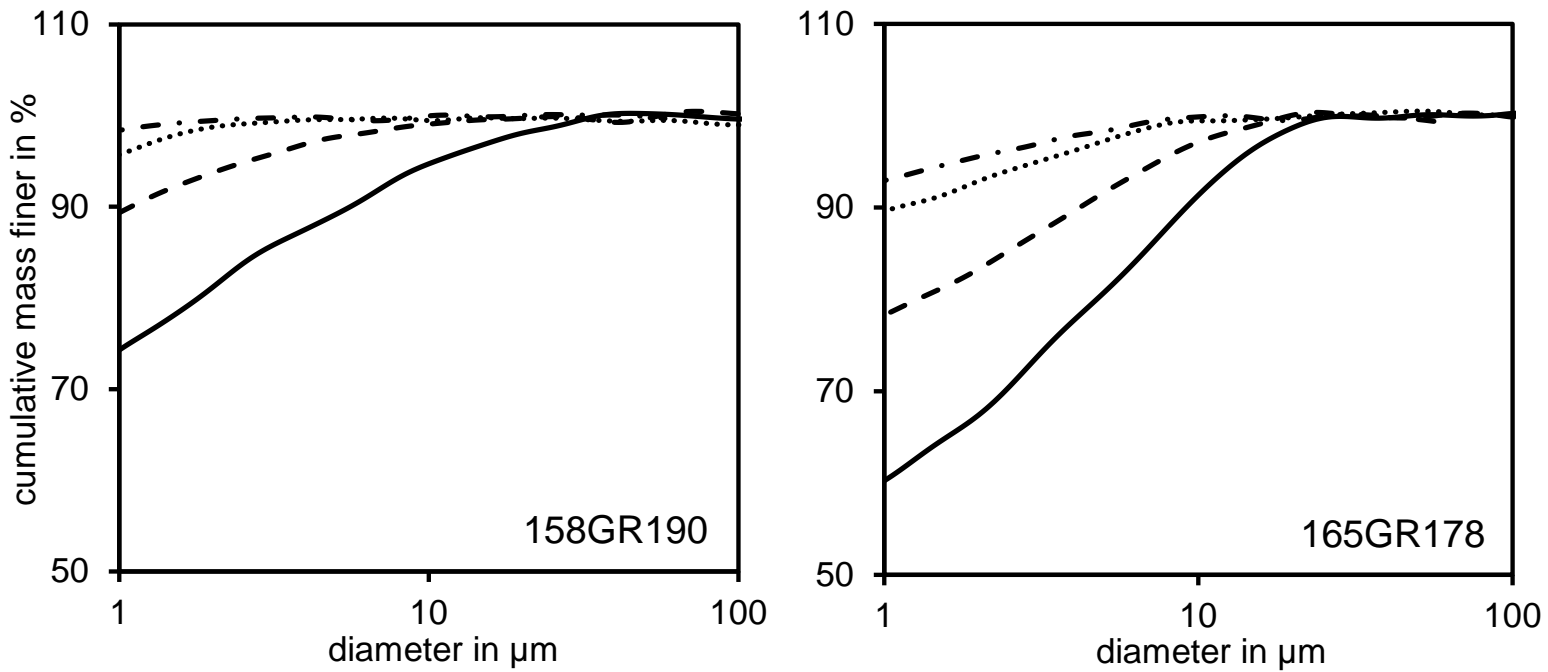

Figure 4.3.7: Analyses of 158GR190 and 165GR178 at $70 \%$ amplitude with 2 min (--), $5 \mathrm{~min}(--), 10 \mathrm{~min}(\cdots)$ and $15 \mathrm{~min}(---)$ ultrasonic pre-treatment

Similar to the treatment with $50 \%$ amplitude all experiments verify that a longer treatment time lead to a continuous reduction in particle sizes. However, with the higher amplitude of $70 \%$ it is hardly possible to differ samples treated for 10 and for $15 \mathrm{~min}$. 
$100 \%$ of amplitude

Fig. 4.3.8 depicts the results of the before mentioned two types of silica treated with $100 \%$ of amplitude for 2, 5, 10 and 15 min with ultrasound. The concentration of particles (cumulative mass finer) is plotted against the calculated diameters.
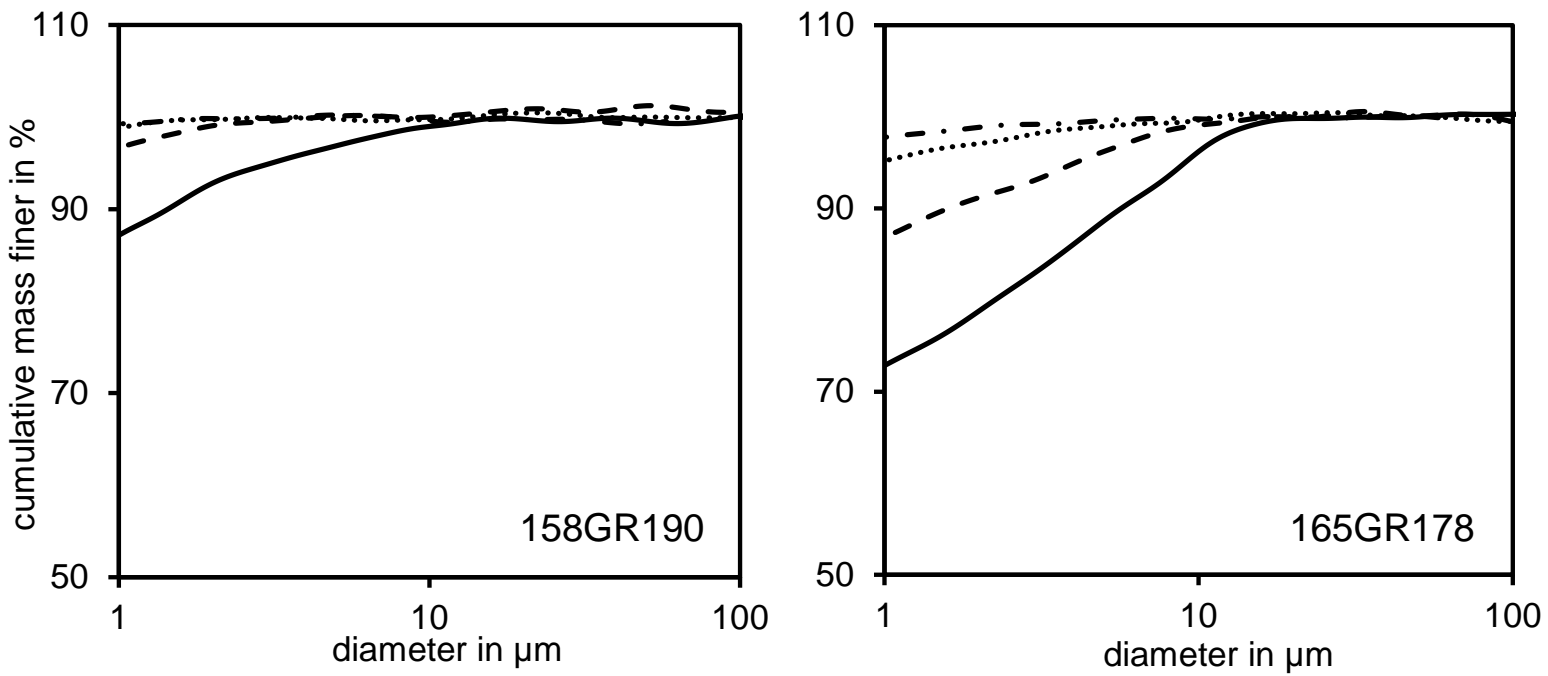

Figure 4.3.8: Analyses of 158GR190 and 165GR178 at $100 \%$ amplitude with 2 min (-), 5 min (- - -), $10 \mathrm{~min}(\cdots)$ and $15 \mathrm{~min}\left(-_{-}-\right)$ultrasonic pre-treatment

It is shown that an amplitude of $100 \%$ corresponds to the highest energy input. It is clearly visible that it is easier to reduce the size of 158GR190 in comparison to 165GR178. Especially in the case of 158GR190 there is hardly any difference between 5, 10 and 15 min of treatment. Additionally, different types of silica cannot be distinguished anymore when ultrasound is applied for 10 min or longer at $100 \%$ amplitude.

\section{Conclusion for the ultrasonic treatment}

The results of the ultrasonic pre-treatment of different types of silica rise the presumption that this sample preparation method is a promising approach to fulfill the task. To choose the most suitable combination of amplitude and time of the sonotrode, different aspects have to be taken into consideration. On the one hand the results have to be highly repeatable and it has to be possible to distinguish between different types of silica. On the other hand the wear of the sonotrode has to be taken into consideration. The higher the amplitude and the longer the treatment time, the faster wear at the sonotrode occurs. The results showed that an amplitude of $30 \%$ is too low to prepare the samples sufficiently whereas an amplitude of $100 \%$ even after a short time (in the case of 158GR190) results in a strong reduction in particle sizes. This includes a risk that different types of silica cannot be satisfactorily distinguished anymore. Finally, two combinations seem to be most suitable to meet the requirements: $70 \%$ / 5 min and $50 \% / 10 \mathrm{~min}$. The higher amplitude thereby increases turbulences inside the suspension during the treatment and therefore guarantee a more homogeneous treatment. Additionally, 5 min preserve the sonotrode from a too fast wear of the sonotrode. Therefore, it was decided to choose an amplitude of $\mathbf{7 0} \%$ for $\mathbf{5}$ min as sample preparation.

\section{First repeatability and evaluation}

To check the repeatability in first place both types of silica (158GR190 and 165GR178) were prepared three times. Therefore, $5 \mathrm{~g}$ of silica were mixed with $50 \mathrm{ml}$ of demineralized water, treated via ultrasound with an amplitude of $70 \%$ for 5 min while being cooled and afterwards tested in accordance with the default settings. The results are shown in Fig. 4.3.9. 


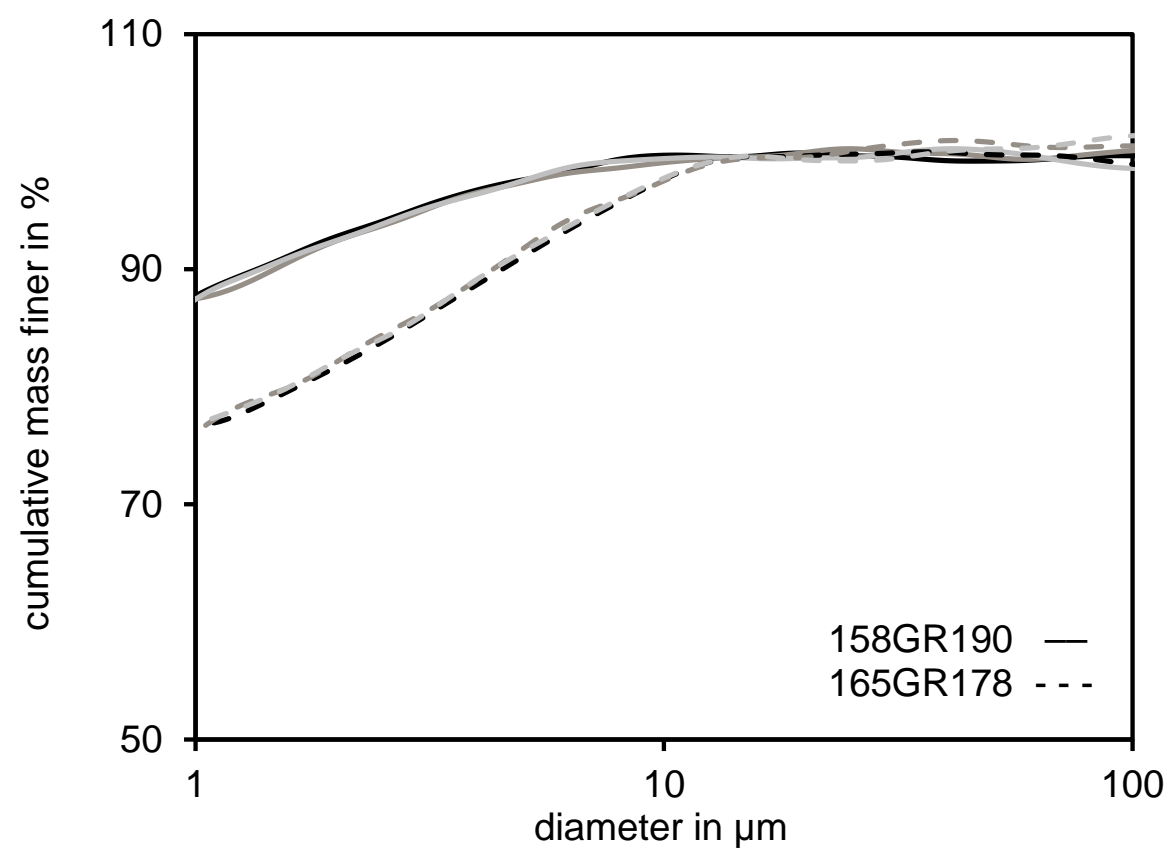

Figure 4.3.9: Sedimentation analyses of 158GR190 (-) and 165GR178 (- -) after 5 min of ultrasonic treatment with an amplitude of $70 \%$

It can be seen that the repeated measurements run almost on top of each other and it is possible to distinguish between different types of silica. To evaluate the final results the amount of residual particles at different diameters are compared as seen in Tab. 4.3.3.

Table 4.3.3: Evaluation of residual amounts of particles bigger than certain diameters

\begin{tabular}{cccccc} 
& $\%>\mathbf{1} \boldsymbol{\mu m}$ & $\%>\mathbf{2} \boldsymbol{\mu m}$ & $\%>\mathbf{5} \boldsymbol{\mu m}$ & $\%>\mathbf{1 0} \boldsymbol{\mu m}$ & $\%>\mathbf{1 5} \boldsymbol{\mu m}$ \\
\hline 158GR190 & 12.2 & 7.4 & 2.6 & 0.6 & 0.4 \\
165GR178 & 23.2 & 17.6 & 8.7 & 2.6 & 0.4 \\
\hline $\boldsymbol{\Delta}$ & 11.0 & 10.2 & 6.2 & 2.0 & 0.0 \\
\hline
\end{tabular}

To distinguish the results of both types of silica the difference between 165GR178 and 158GR190 $(\Delta)$ is compared. It can be seen that the smaller the diameter, the easier it is to differ the outcome. Above a size of $10 \mu \mathrm{m}$ hardly any particles are detected. Therefore, it was decided to use the amount of particles bigger than $\mathbf{1} \boldsymbol{\mu m}$ as a final value to compare future results. Due to the fact that the results of the sedimentation analysis are planned to be correlated to the in-rubber macro-dispersion quality of rubber compounds in the upcoming chapter 5 , the values of particles bigger than $\mathbf{2} \boldsymbol{\mu m}$ are recorded as well. This number corresponds to the distinction between macro- and micro-dispersion in accordance with the ASTM definition [10].

\section{Adjustment of the pre-treatment}

During additional test on other types of silica it turned out that several highly dispersible types of silica could not be distinguished well with the chosen sample preparation. Therefore, the ultrasonic treatment time was slightly adjusted from $5 \mathrm{~min}$ to $4 \mathrm{~min}$. Fig. 4.3 .10 depicts the relative amount of particles bigger than 1 and $2 \mu \mathrm{m}$ of 104GR175, 159GR204 and 122GR195 after 5 min and after 4 min of ultrasonic treatment (70\% amplitude). 


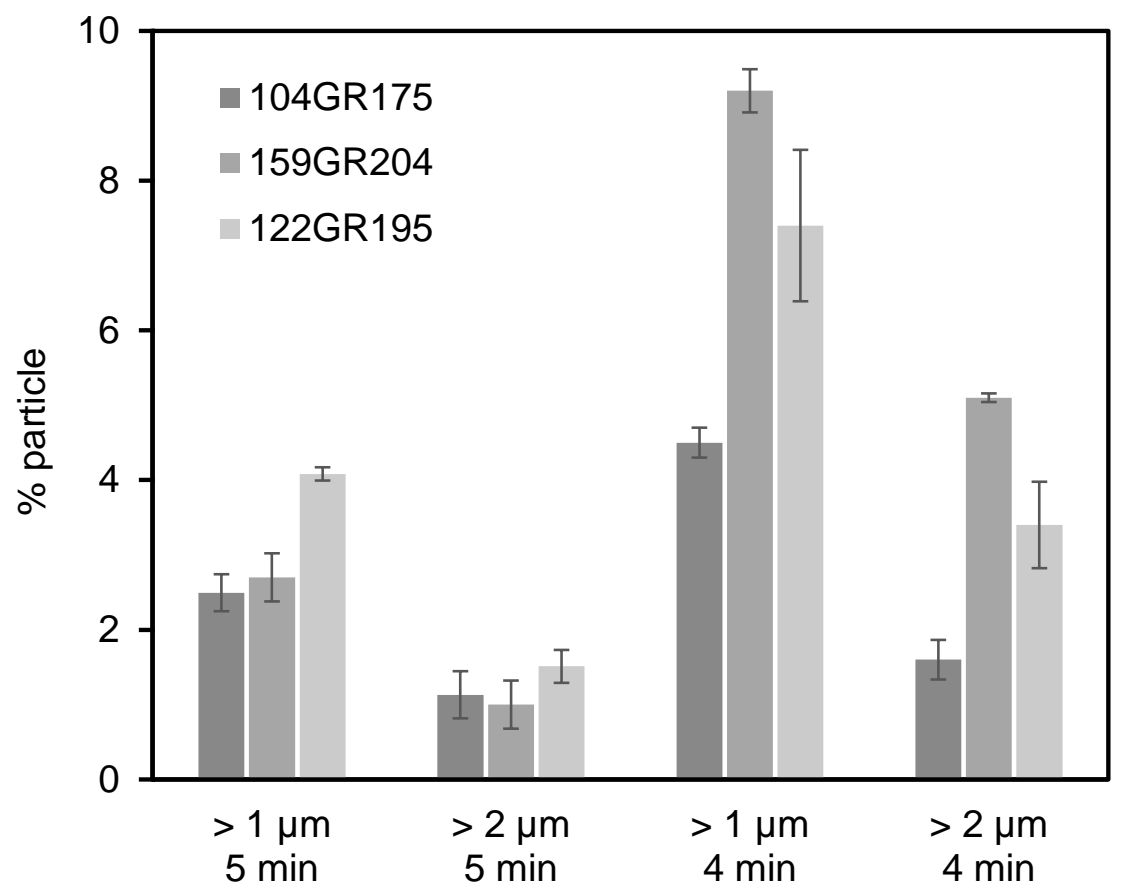

Figure 4.3.10: Comparison of the impact of 4 and 5 min of sonification on the amount of particles bigger than 1 and $2 \mu \mathrm{m}$ of three different types of silica

It can be seen that after 5 min of treatment it is hardly possible to differ between the three types of silica neither at $>1 \mu \mathrm{m}$ nor at $>2 \mu \mathrm{m}$ whereas after $4 \mathrm{~min}$ all samples could be distinguished at $>1 \mu \mathrm{m}$ as well as at $>2 \mu \mathrm{m}$. Therefore, it was decided to change the ultrasonic pre-treatment for all upcoming measurements to $4 \mathrm{~min}$ at $\mathbf{7 0} \%$ amplitude. It is conspicuous that the relative particle size distributions of the three types of silica to each other change with the time of sonification. The 122GR195 for instance shows the highest amount of residual particles bigger than $1 \mu \mathrm{m}$ after $5 \mathrm{~min}$ of treatment $(4.1 \%)$ whereas after 4 min the 159GR204 demonstrates the worst result $(9.2 \%)$. Therefore, it can be concluded that the speed of cluster breakage differs from silica to silica which might also affect the in-rubber dispersion process.

\section{Cooling of the suspension during ultrasonic treatment}

It is claimed [4] that the temperature of the suspension during the ultrasonic treatment influences the effectiveness of cavitation and cluster size reduction as described in chapter 4.1. Therefore, a simple test was performed to check the influence of cooling on the final result. As a standard, $5 \mathrm{~g}$ of a silica granule (215GR210) with $50 \mathrm{ml}$ of water was prepared via ultrasound for 4 min with $70 \%$ amplitude while the beaker was halfway immersed into a cooling bath of $5{ }^{\circ} \mathrm{C}$. The same sample was prepared excluding the cooling bath at room temperature. Both settings were performed three times and compared.

The temperature of the suspensions was directly measured after sonification by means of a sensor attached to the ultrasound generator. The cooled samples reached a temperature between 30 and $40^{\circ} \mathrm{C}$ while the uncooled samples went up to $70^{\circ} \mathrm{C}$. Fig. 4.3 .11 shows the effect of cooling on the relative amount of clusters bigger than 1 and $2 \mu \mathrm{m}$ after sonification. 


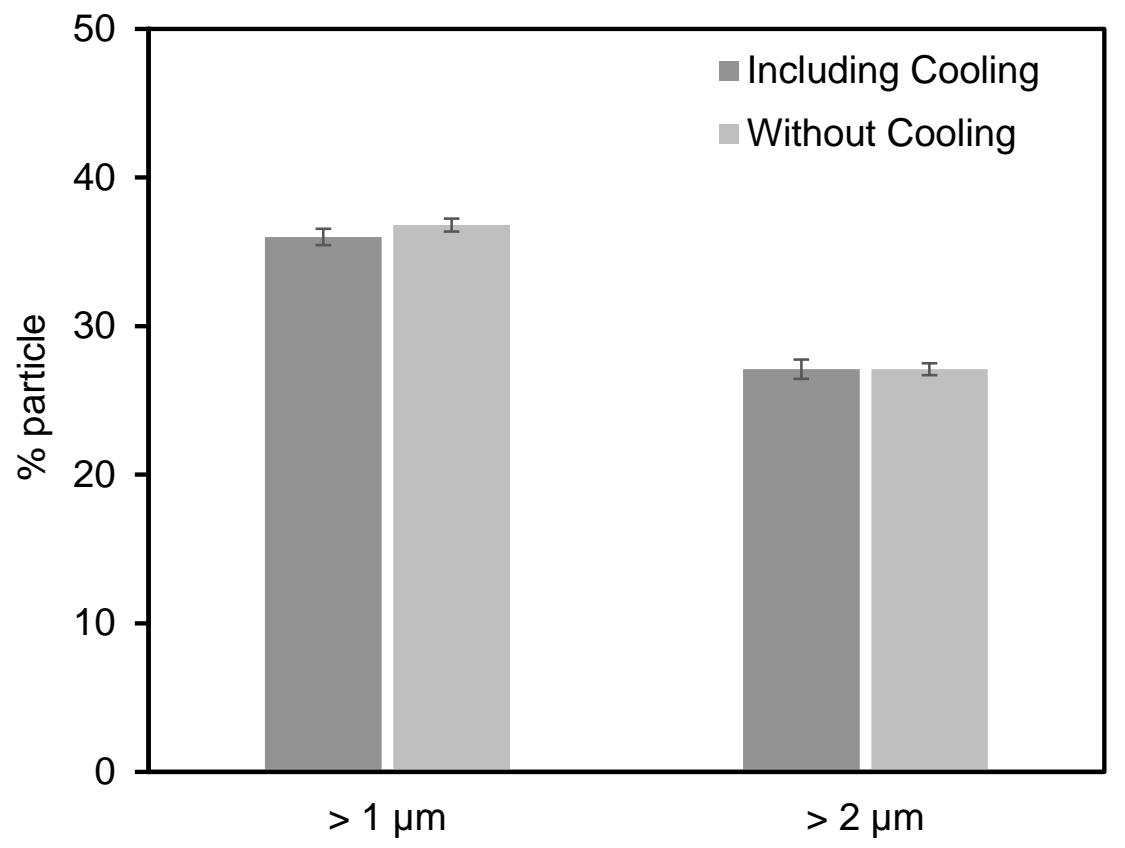

Figure 4.3.11: The effect of cooling on the amount of particles bigger than 1 and $2 \mu \mathrm{m}$

It turned out that cooling of the suspension during ultrasonic treatment does not influence the final result at all. Nevertheless, the cooling is important for the following reason. The suspension inside the analytical device (SediGraph) is measured at $35^{\circ} \mathrm{C}$ to guarantee a specific viscosity and a correct particle size calculation. Hence, the suspension prepared without cooling needs to be cooled down for a certain period of time before the actual measurement can be started. This results in roughly 10 to 15 min of additional measurement time and is unwanted for a daily lab routine. Additionally, handling the beaker at $+60^{\circ} \mathrm{C}$ is undesired and includes a risk of burn injuries. Therefore, it is recommended to include the cooling procedure during the sample preparation. All following ultrasonic sample preparations were conducted including cooling of the suspension.

\section{Position of Sonotrode}

To check if the position of the sonotrode influences the final result, four different variations were tested. Firstly, the sonotrode was positioned central inside the beaker with a depth of $50 \mathrm{~mm}$ (a) as recommended by the supplier [11] which corresponds to the standard procedure. Additionally, the immersion depth was varied, once to $60 \mathrm{~mm}$ (b) and once to $40 \mathrm{~mm}$ (c). Finally, the sonotrode was decentred and aligned close to the sidewall of the beaker (d) as can be seen in Fig. 4.3.12.

a)

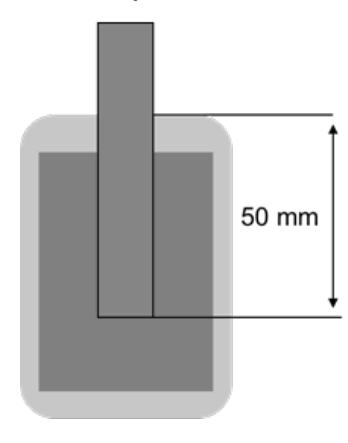

b)

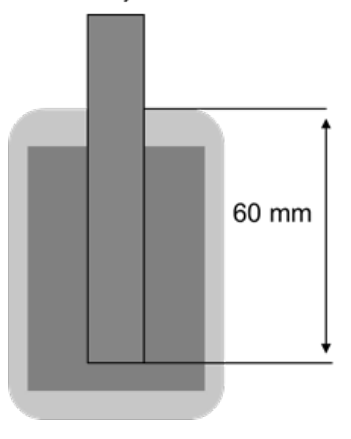

c)

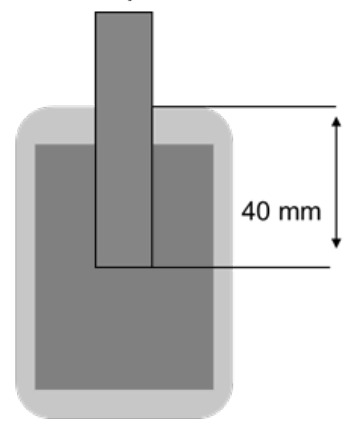

d)

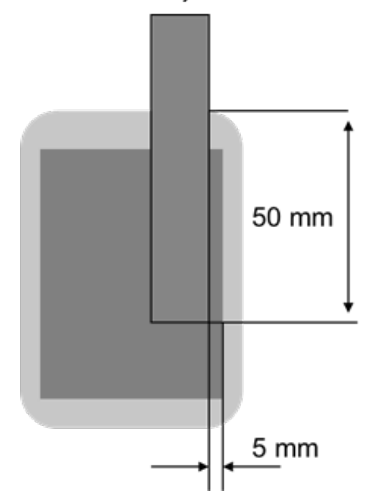

Figure 4.3.12: Schematic view of the different positions of the sonotrode inside the beaker 
$5 \mathrm{~g}$ of a silica granule (215GR210) with $50 \mathrm{ml}$ of water was pre-treated via ultrasound for $4 \mathrm{~min}$ with $70 \%$ amplitude and measured three times within each position. Fig. 4.3.13 depicts the relative amount of clusters bigger than 1 and $2 \mu \mathrm{m}$ for all four positions. Each measurement was repeated three times.

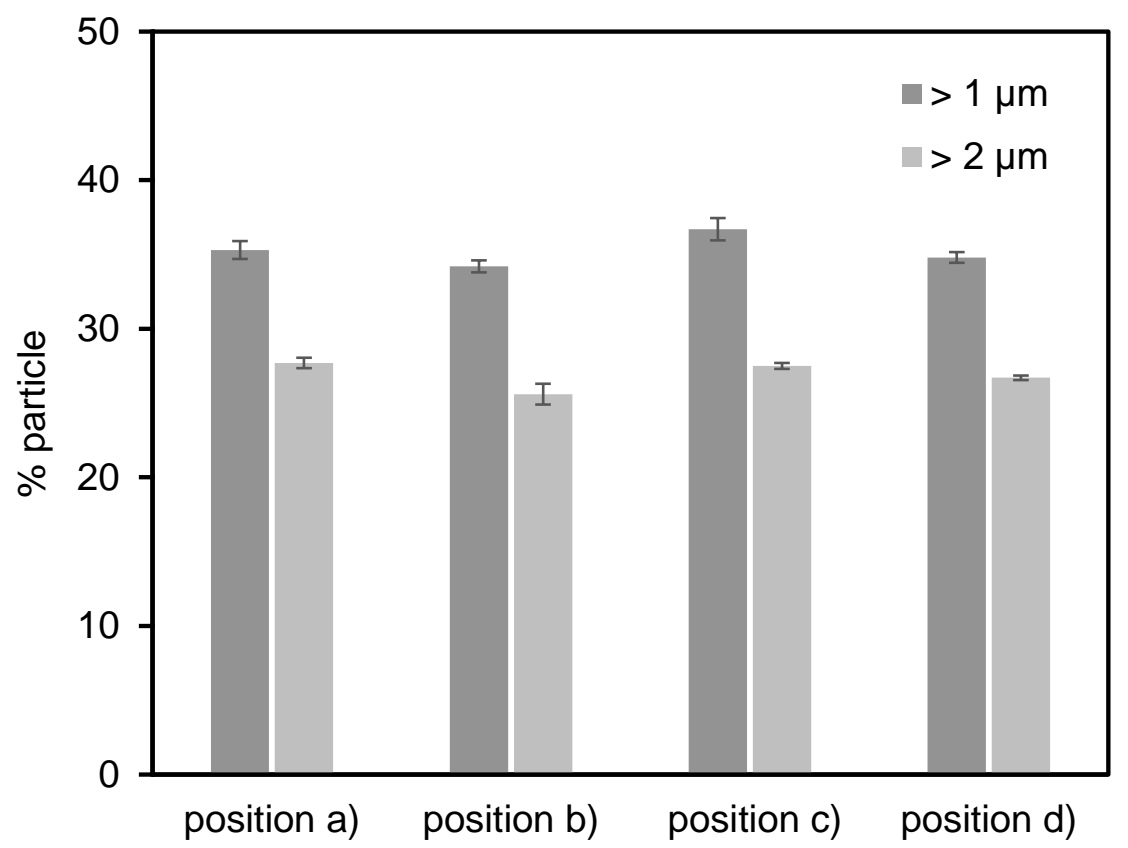

Figure 4.3.13: The effect of the position of the sonotrode a) to d) on the amount of particles bigger than 1 and $2 \mu m$

It is shown that all detected amounts of particles are on the same level within the measurement error, independent of the position of the sonotrode. The suspension is sufficiently mixed during the ultrasonic treatment due to the caused flow by means of the cavitation. This experiment also confirms that cavitation only occurs on the tip of the sonotrode and neither the side of the sonotrode, nor the distance to the bottom of the beaker play a role in reducing the cluster sizes. To guarantee consistent conditions the position of the sonotrode is set to the standard position a) for all following experiments. 


\section{Influence due to inaccurate concentration}

Previous experiments showed already that $5 \mathrm{~g}$ of silica with $50 \mathrm{ml}$ of water is needed to achieve a sufficient level of absorption of the X-ray beam. Additionally, the following experiments were made to point out the necessity of an accurate concentration by use of the optimized sample preparation. Therefore 4,5 and $6 \mathrm{~g}$ of silica (215GR210) were prepared via ultrasound for 4 min with $70 \%$ amplitude and measured three times (Fig. 4.3.14).

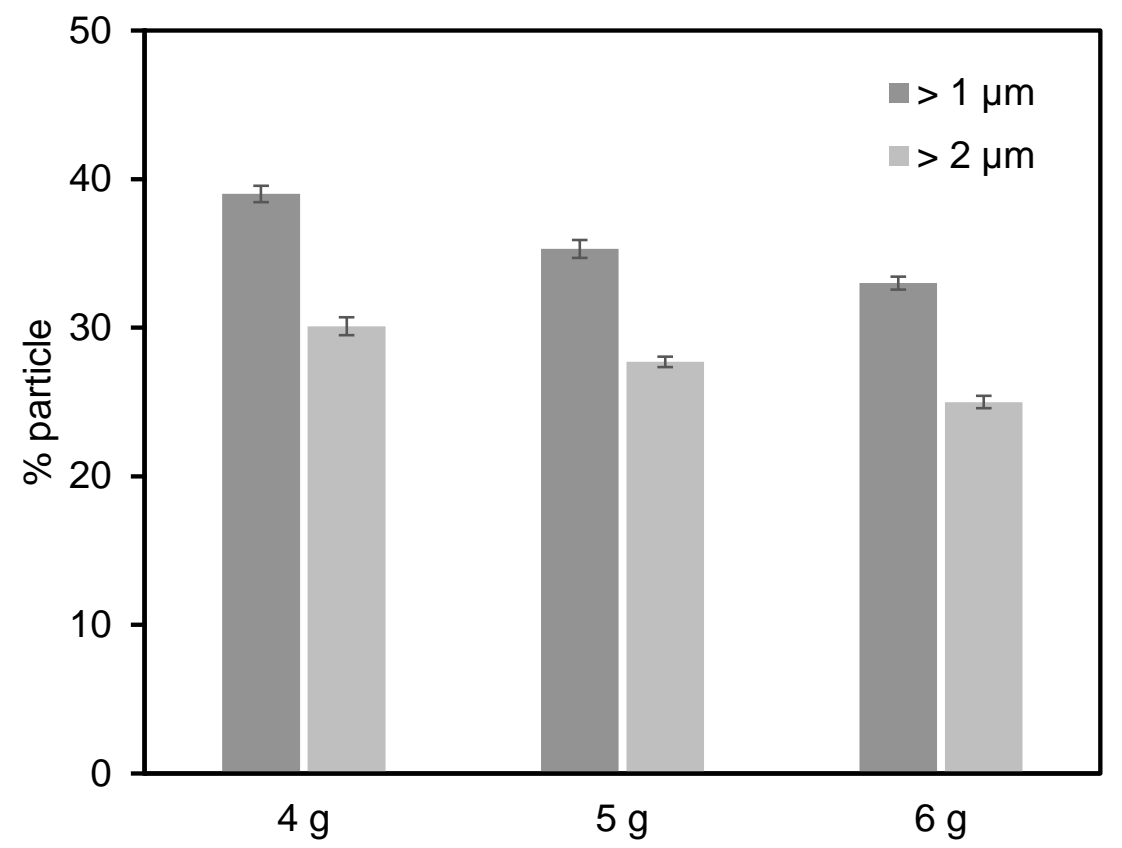

Figure 4.3.14: The effect of the silica concentration 4, 5 and $6 \mathrm{~g}$ on the amount of particles larger than 1 and $2 \mu \mathrm{m}$

Besides the fact that $4 \mathrm{~g}$ of silica results in an insufficient level of absorption in accordance with the recommendation of the manufacturer it was still possible to measure and evaluate the results. It can be seen that a higher concentration leads to a smaller amount of residual large clusters. One explanation can be based on the fact that particles are accelerated by means of the cavitation. A higher concentration of particles causes a higher probability of collisions between silica clusters which can, next to the cavitation itself, also result in a reduction of particle sizes. 


\section{Speed of measurement (analysis type)}

The speed of measurement respectively analysis type can be adjusted to three different settings. While selecting the "high speed" mode the cell moves upwards in opposite direction of particle settling by gravity. Therefore, particles are detected faster but this causes a reduced resolution. In the "high resolution" mode, a high resolution can be achieved but the measurement takes longer. The "standard" mode represents a compromise in between a decent speed and resolution. A silica granule (215GR210) was prepared via ultrasound for 4 min with $70 \%$ amplitude and measured three times with these three possible analysis modes.

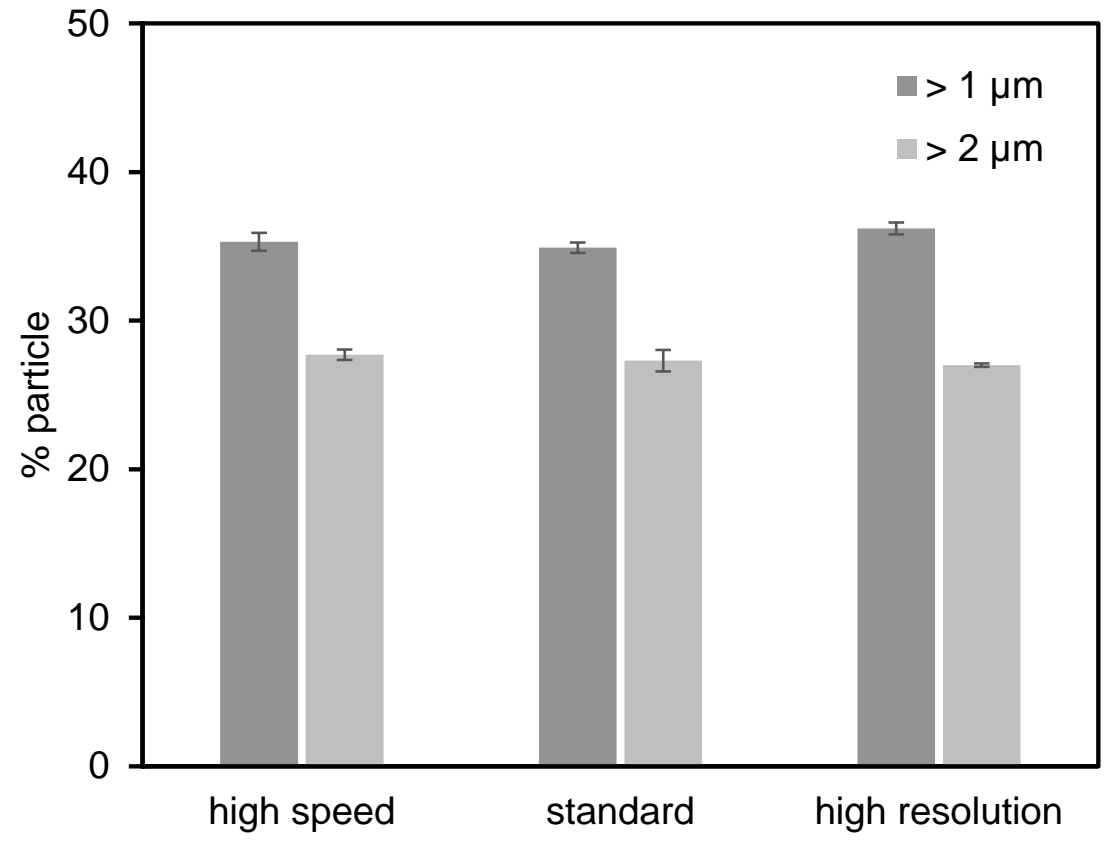

Figure 4.3.15: Effect of the measurement speed on the amount of particles bigger than 1 and $2 \mu \mathrm{m}$

Fig. 4.3.15 shows the influence of the three different analysis types on the residual amount of cluster sizes bigger than 1 and $2 \mu \mathrm{m}$. Due to the fact that no difference is visible, the "high speed" analysis is chosen for an appropriate lab routine.

\section{$X$-ray Intensity}

The Intensity of the X-ray beam can be adjusted in two ways, the standard mode where the intensity is set to "normal" and the "low intensity" mode. The latter is especially suitable for materials which absorb the X-ray beam only to a low extend. To evaluate the influence of the X-ray intensity a silica granule (165GR178) was rubbed through a $500 \mu \mathrm{m}$ sieve, mixed with $50 \mathrm{ml}$ of water and subsequently treated via ultrasound for $4 \mathrm{~min}$ with $70 \%$ amplitude. For the "normal" mode $5 \mathrm{~g}$ of silica was chosen whereas for the "low intensity" mode it was necessary to adjust the concentration due the fact that the X-ray beam was fully absorbed by the silica. It turned out that $2.5 \mathrm{~g}$ of silica mixed with $50 \mathrm{ml}$ of water resulted in a reduction of baseline of $32 \%$ which is in accordance with the recommendation of the supplier. Each experiment for both settings was performed three times and the amount of clusters bigger than 1 and $2 \mu \mathrm{m}$ was evaluated. 


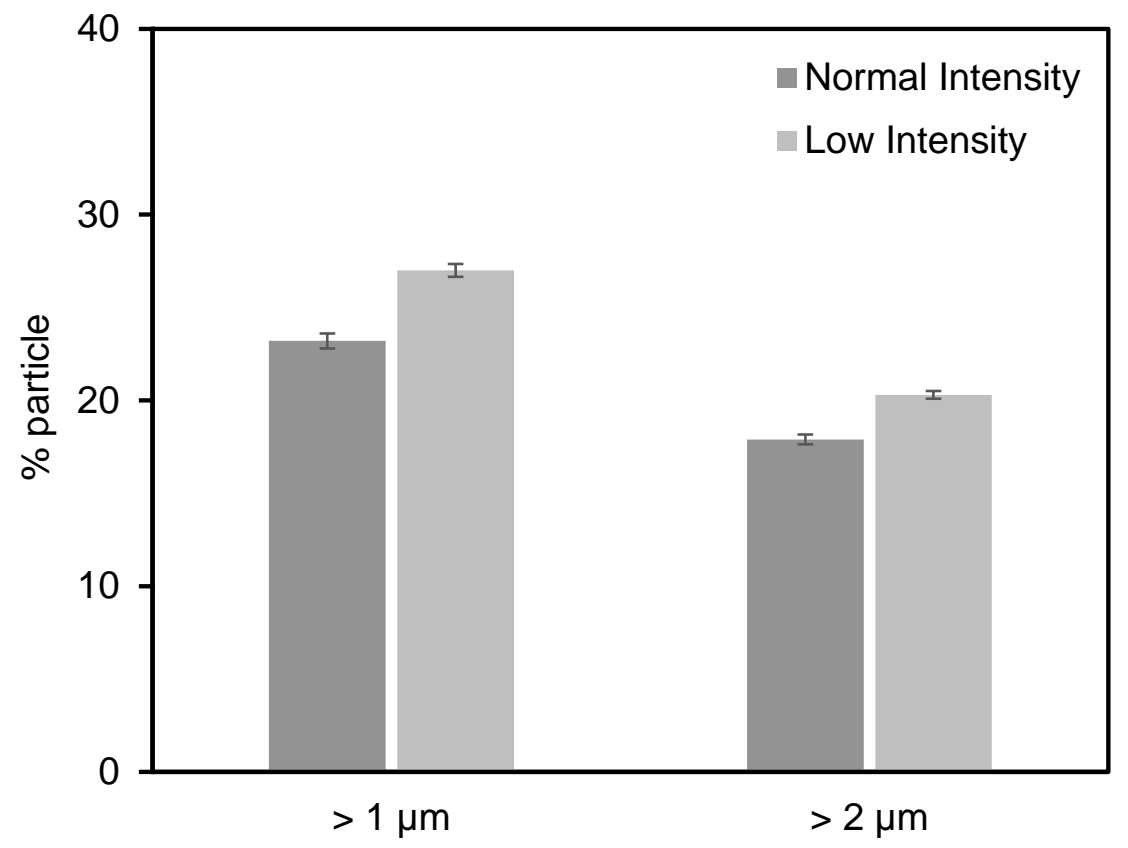

Figure 4.3.16: Effect of the X-ray intensity on the amount of particles bigger than 1 and $2 \mu m$

Fig. 4.3.16 depicts the influence of the intensity to the final result. It can be seen that it was possible to perform the measurement with both settings and that the lower intensity show higher amounts of large clusters. However, this can be explained by the adjusted silica concentration for the measurement and corresponds to the results seen beforehand where the concentration was varied. Both modes show a high repeatability with no clear tendency to one setting. With regard to the measurement time the "normal" mode takes 8 min whereas the "low intensity" mode needs 21 min to perform the measurement. Therefore, it was decided to keep the standard X-ray intensity for all experiments.

\section{Repeatability and Reproducibility}

All investigations on the method development of the sedimentation analysis including the sample preparation and variation of the process parameter lead to the following final setup:

- $\quad$ Analysis Type:

high speed

- $\quad$ Analysis Temperature: $35^{\circ} \mathrm{C}$

- $\quad$ Analysis Liquid

demineralized water

- X-Ray Intensity:

Normal

- Density Silica:

$2.0 \mathrm{~g} / \mathrm{cm}^{3}$

- $\quad$ Concentration:

$50 \mathrm{ml} \mathrm{H}_{2} \mathrm{O}+5 \mathrm{~g}$ silica

- Ultrasound:

$70 \%$ amplitude for 4 min in a cooling bath at $5{ }^{\circ} \mathrm{C}$

To prove the repeatability and reproducibility of this measurement, 6 different silica (two types of powders, granules and micro-pearls) with a variety of analytical parameters were investigated. Three different operators tested each silica four times (twelve measurements per type of silica). Based on this, the standard deviation was determined (Fig. 4.3.17). 


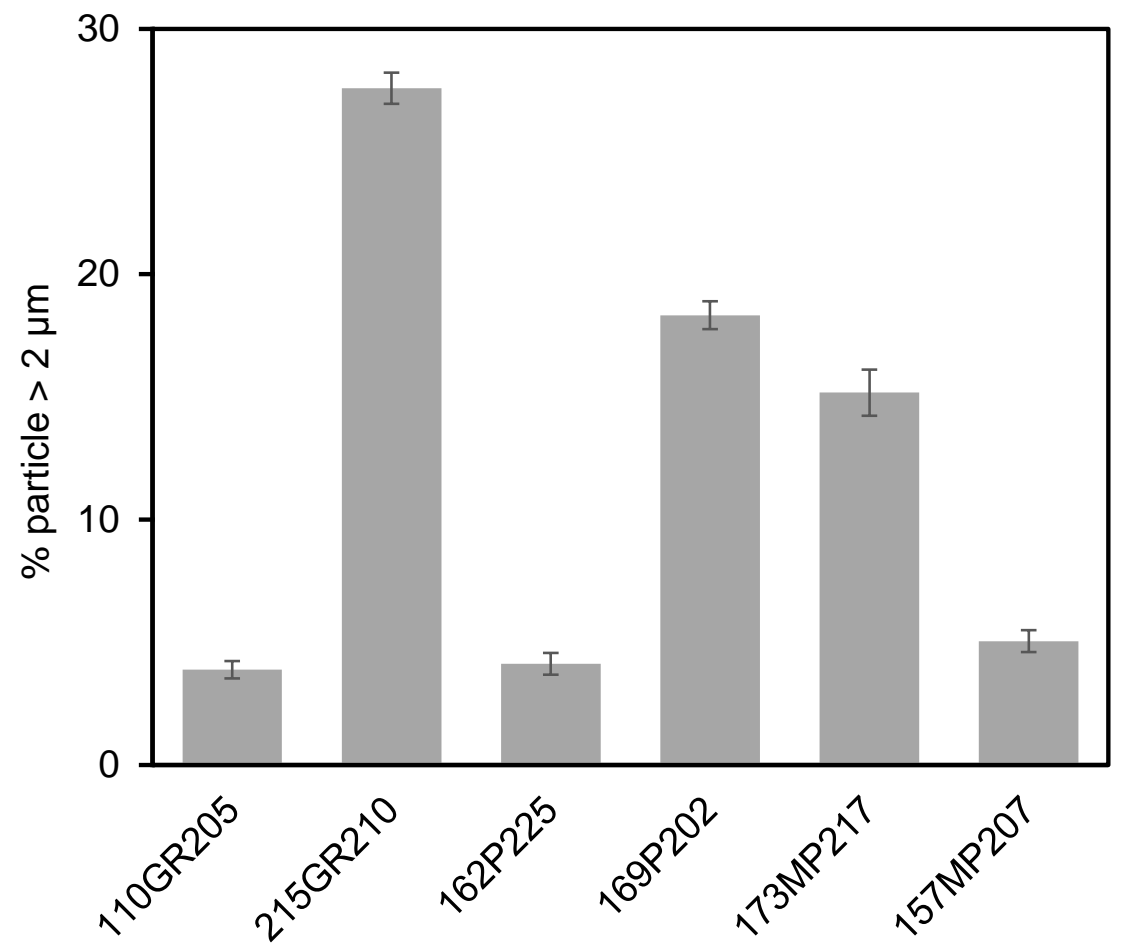

Figure 4.3.17: Reproducibility test of 6 different types of silica conducted by 3 operators (in total 12 measurements per grade)

It can be seen that deviations of results within each type of silica are comparable low with a maximum standard deviation of approximately $1 \%$. There are three silica which contain only a very low amount of particles larger than $2 \mu \mathrm{m}$ whereas the three others show a significantly higher amount of those. The silica 215GR210 contains the most. With regards to the general requirements for method development in chapter 4.1 it can be said that the sedimentation analysis with given final settings show a sufficient repeatability and reproducibility with a decent standard deviation. It is possible to distinguish between different types of silica and also between different silica with comparable dosage forms.

\section{Storage effect}

To investigate the effect of storage time and conditions a final storage experiment was conducted (final settings). Therefore a freshly produced silica granule (168GR180) was tested and artificially aged under the following conditions (Tab. 4.3.4):

Table 4.3.4: Conditions of the storage test for the sedimentation analysis

\begin{tabular}{cc} 
Condition & Time \\
\hline Freshly produced & $0 \mathrm{~h}$ \\
$105^{\circ} \mathrm{C}$ (oven) & $24 \mathrm{~h}$ \\
\hline room temperature (RT) & 1 week \\
$50^{\circ} \mathrm{C}$ (oven) & 1 week \\
$-18^{\circ} \mathrm{C}$ (freezer) & 1 week \\
\hline $\mathrm{RT}$ & 1 month \\
$50^{\circ} \mathrm{C}$ (oven) & 1 month \\
$-18^{\circ} \mathrm{C}$ (freezer) & 1 month \\
\hline
\end{tabular}


All samples were stored in their original dosage form (GR) and crushed just before the sample was pre-treated via ultrasound. Each experiment was repeated 3 times and the standard deviation was determined. Fig. 4.3.18 depicts the results of the percentage of particles bigger than $2 \mu \mathrm{m}$ under different storage times and conditions.

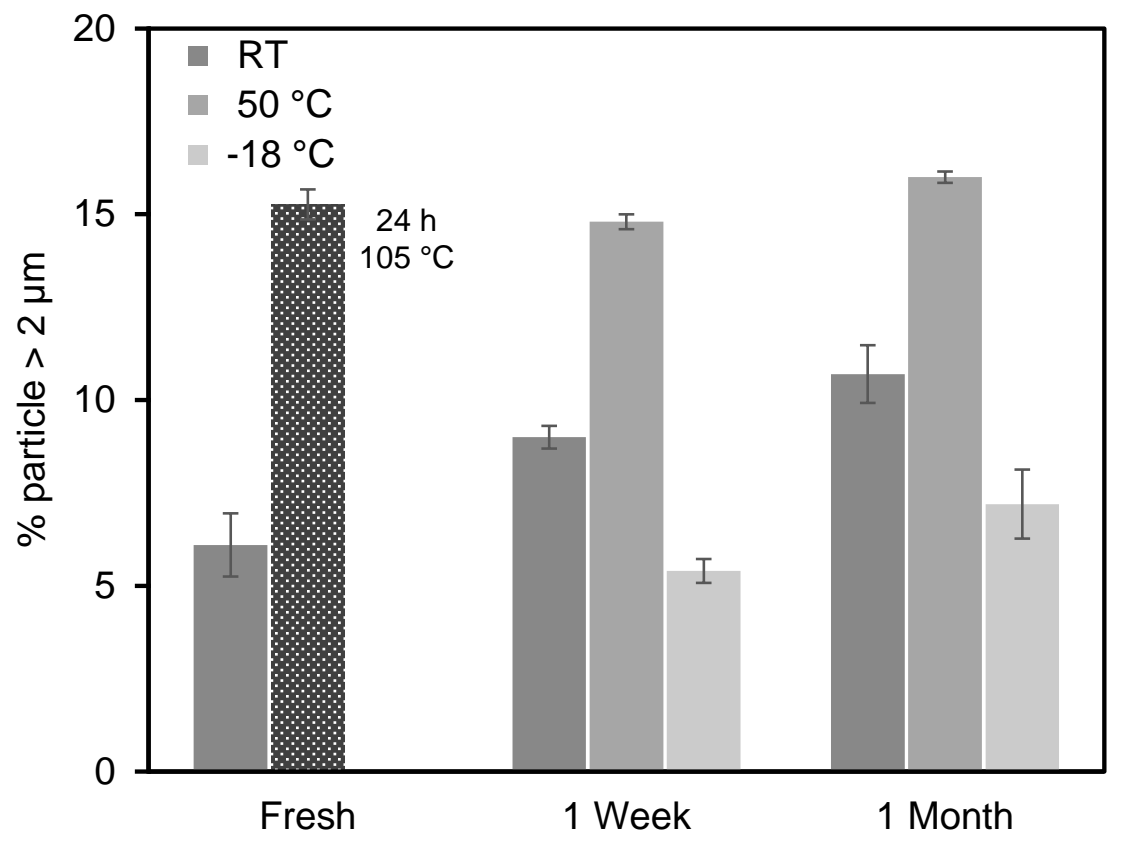

Figure 4.3.18: Effect of different storage conditions on the amount of particles bigger than $2 \mu m$

As a reference, the fresh produced silica was examined. To check if there is an effect due to storage, silica samples firstly were kept in an oven at $105^{\circ} \mathrm{C}$ for 24 hours. It can be seen that the storage at higher temperatures leads to a greater amount of particles bigger than $2 \mu \mathrm{m}$. This means it becomes more difficult to break down the clusters. One explanation can be that the removal of free water at the silica surface results in additional stable physical links between particles (higher amount of "free" silanol groups forming hydrogen bondings).

The same value can be reached by treating silica in an oven at $50^{\circ} \mathrm{C}$ for 1 week and 1 month. Therefore it seems to be most likely that a possible maximum value due to storage can be reached which remains stable at a certain point.

Keeping the silica at room temperature (RT) for 1 week and 1 month shows a clear tendency to bigger particle sizes with increasing storage time. However, even after one month the value of the $105^{\circ} \mathrm{C} / 24 \mathrm{~h}$ measurement was not reached.

Storing silica inside a freezer seems to prevent changing its properties regarding the sedimentation analyses, the values are the same as for the reference.

As a conclusion, it can be said that for an elimination of the effect of storage either silica has to be tested shortly after the production, or stored inside a freezer or always pre-treated inside an oven at higher temperature for a certain time e.g. at $105^{\circ} \mathrm{C}$ for $24 \mathrm{~h}$.

\subsubsection{Concerns and Reservations}

The use of the sedimentation method to measure the particle size distribution of silica includes several assumptions and possible sources of error which have to be taken into consideration when interpreting the final results. 
One requirement for using Stokes' Law to calculate particle sizes during sedimentation is to have a Reynolds number of less than 0.3 [5]. As a matter of fact the calculated Reynolds number with given information about the suspension corresponds to 1.04. Therefore, a turbulent flow can occur and hence Stokes' law is no longer valid. However, this approximation is used knowing that it might have a stronger influence to the final results when comparing highly different practical sizes.

Another condition is that the fluid has an infinite extend. Hence, a low volume concentration of maximum $1 \% \mathrm{v} / \mathrm{v}$ is required. In the present work a concentration of almost $10 \%$ was chosen to be able to conduct the experiments. Additionally, the sidewall to sidewall distance supposed to be at least $5 \mathrm{~mm}$ to reduce wall effects [5]. However, this distance inside the measurement cell only corresponds to $3 \mathrm{~mm}$.

Finally, the wear of the sonotrode for the sample preparation has to be taken into account. To investigate the influence of the sonotrode one type of silica granule (215GR210) was prepared and measured in accordance with the final test settings for the sedimentation analysis. Four different sonotrodes were chosen, three new ones (New 1, New 2 and New 3) to check the influence due to flocculation of production and an old one which was often used and showed heavy wear to determine the influence due to wear (Fig. 4.3.19). Each test was performed three times.

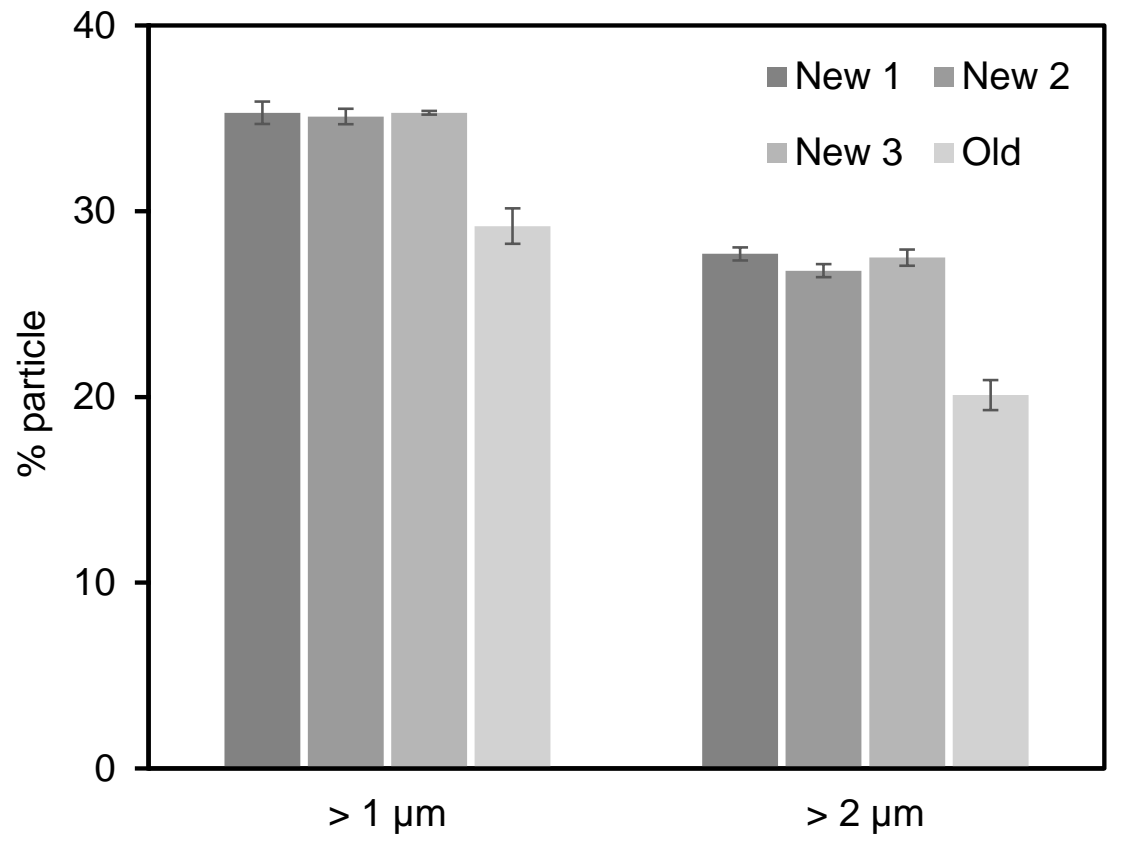

Figure 4.3.19: Effect of different sonotrodes on the amount of particles bigger than 1 and $2 \mu \mathrm{m}$

It can be seen that the results of the three new sonotrodes are on the same level with a comparable low deviation. However, the old sonotrode shows a pronounced divergence in comparison to the new ones. Therefore, it is essential to monitor the degree of wear on a regular base. It is noticeable that the samples prepared with the worn sonotrode do contain a lower amount of particles bigger than 1 and $2 \mu \mathrm{m}$ which corresponds to a higher energy input and more effective size reduction. Due to the rough surface of the worn sonotrode the total surface area, where cavitation occurs, is enlarged. Assuming that the energy input is dependent on the total surface a more effective size reduction can be explained. Different possibilities to monitor the wear of the sonotrode were already shown in chapter 4.1. 


\subsubsection{Summary and Conclusion}

This chapter presents a new analytical method to determine the particle size distribution of silica via X-ray absorption during sedimentation after an ultrasonic treatment (defined energy input). All parameters which might influence the final outcome of the measurement were tested and an optimal measurement procedure including sample preparation was determined. The smaller the particle size, the slower they settle by means of gravity. The measurement system is limited to particle sizes of 0.1 to $300 \mu \mathrm{m}$, though detecting clusters smaller than $1 \mu \mathrm{m}$ takes roughly three hours which limits the use for a daily lab routine. However, with regard to the macro-dispersion qualities where only particles on a micro-scale are taken into account, the measurement can be stopped after going down to $1 \mu \mathrm{m}$. This results in a tremendous reduction of measurement time. The sedimentation analysis shows a proper repeatability and a sufficient differentiation between different types of silica. The sample preparation via ultrasound is very sensitive. A precise concentration of the sample is needed to avoid. Furthermore, the wear of the sonotrode has a strong effect on the sample preparation. Therefore, it is indispensable to properly monitor the quality of the sonotrode.

As a conclusion, it can be said that the sedimentation analysis does not meet all requirements in accordance with the general basics for method development as described in chapter 4.1. Especially the storage of the silica influences the final outcome. It is recommended to reduce this influence by storing the samples inside an oven under defined conditions. Beside this, the newly developed method can be seen as a suitable analytical measurement system.

\subsubsection{References}

[1] Y. Chevallier et al., US 2003/0118500 A1, precipitated silica used as reinforcing filler for elastomers, US 2003/0118500 A1 (2003)

[2] Micromeritics, SediGraph III Plus - Operator Manual V1.01 (2014)

[3] P. A. Webb; C. Orr, Analytical Methods in Fine Particle Technology, Norcross, GA 30093 U.S.A. (1997)

[4] Internal Information, Evonik Resource Efficiency GmbH

[5] M. Rhodes, Introduction to ParticleTechnology - Second Edition, John Wiley \& Sons, Southern Gate, Chichester, England (2008)

[6] A. J. Rowe, Analytical Ultracentrifugation: Techniques and Methods, Chapter 14: Introduction to Differential Sedimentation, Cambridge, UK (2005)

[7] ISO 13317-3:2001 - Determination of particle size distribution by gravitational liquid sedimentation methods - Part 3: X-ray gravitational technique

[8] R. K. Iler, The Chemistry of Silica, John Wiley \& Sons, New York - Chichester - Brisbane - Toronto (1978)

[9] Fritsch $\mathrm{GmbH}$, Advertising brochure: Rotor-Schnellmühle und Schlagkreuzmühle, (2016)

[10] ASTM D3053:2015 - Standard Terminology Relating to Carbon Black

[11] Personal information, BRANSON Ultrasonic Coop. 


\section{Chapter 4.4 - Method Development 3: In-situ Cluster Fragmentation}

During mixing of silica inside a rubber matrix, the particle size of the filler is successively reduced. This procedure is referred to as the dispersion process. Some types of silica are easier to decrease in their cluster size than others. To gain information about the easiness and speed of cluster breakage inside a rubber matrix an approach was made to observe the size reduction during a defined energy input on lab scale.

In order to do so, a suspension of demineralized water and silica circulates inside a closed loop including a steering device to control the velocity speed. Each circulation, this suspension passes an ultrasonic device to reduce the cluster size and a laser diffraction system to measure the particle size distribution. In this way, the reduction of particle size respectively the speed of fragmentation can be recorded in-situ. Different curve characteristics were investigated and evaluated.

The in-situ cluster fragmentation method is mainly dependent on the ultrasonic treatment respectively amplitude and time. In addition, the sample mass, flow velocity and temperature of the suspension play a minor role. A certain influence due to storage conditions could be observed and the wear of the sonotrode has to be monitored. One major concern is the use of the Fraunhofer approximation. Even if the use of this theory leads to more suitable results in comparison to the Mie theory, it does not meet the requirements of the measured size range.

The in-situ cluster fragmentation method was successfully implemented to use it for the characterization of silica samples. A suitable reproducibility is achieved and it is possible to distinguish between different types of silica.

\subsubsection{Introduction}

Different types of silica widely vary in their particle size and distribution. Comparing these absolute sizes of silica clusters after a defined energy input as shown in the previous chapter 4.3 certainly gives indications about their dispersibility. However, no specific conclusion to the easiness of cluster breakage can be drawn without taking the following example into consideration:

Two types of silica are compared. The first sample which has already a small initial particle size distribution can hardly be reduced in size any further by means of an energy input. The second silica sample on the contrary contains initially bigger particle sizes which can be reduced in size much easier. When comparing only the absolute sizes after a defined energy input both samples might possess similar values. Hence, it is necessary to additionally gain information about the relative size reduction during a treatment.

In order to get a better insight into the easiness and speed of cluster breakage relatively to the initial size of different types of silica, the change of the particle size and distribution during fragmentation should be observed. Therefore, the idea of using the lasergranulometry [1] occurred. This technique combines a laser diffraction system as a measurement of the particle size distribution with an ultrasonic treatment to reduce the cluster sizes. For this purpose, silica is dissolved inside a fluid which continuously circulates between the measuring system and the ultrasonic device. During the measurement, the solution is treated with ultrasound while the particle size distribution is permanently recorded. In this way, information about the speed of particle size reduction can be obtained. Fig. 4.4.1 depicts an example of particle size distributions curves of one type of silica at different times during the measurement respectively treatment with ultrasound. The volume density is thereby plotted as a function of particle diameter. 


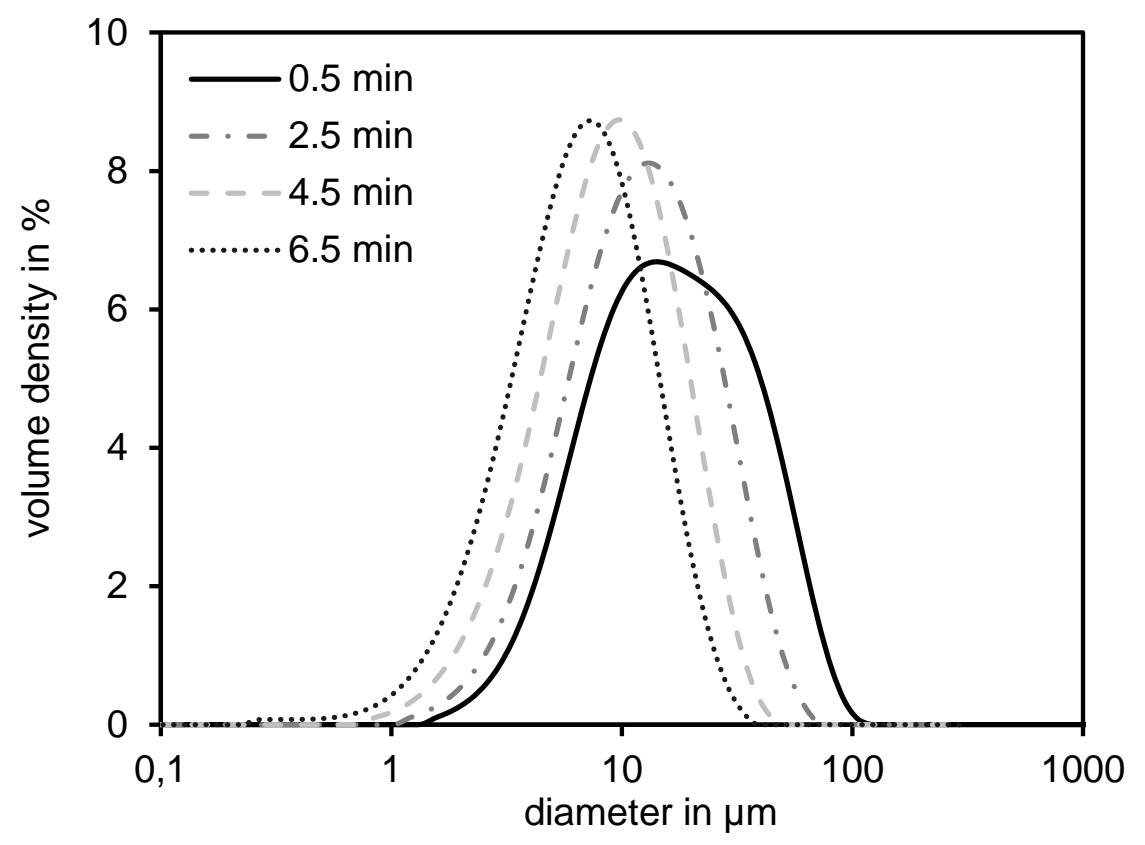

Figure 4.4.1: Particle size distribution curves after different sonification times

With increasing treatment time the particle sizes are reduced and the distribution curves are therefore shifted to smaller diameters. It is described in the literature [2] that the volume weighted mean $D[4 ; 3]$ as described in chapter 4.4 .3 should be evaluated for each measured curve. This value is plotted inversely as a function of time as shown in Fig. 4.4.2. In this way, a positive slope is obtained.

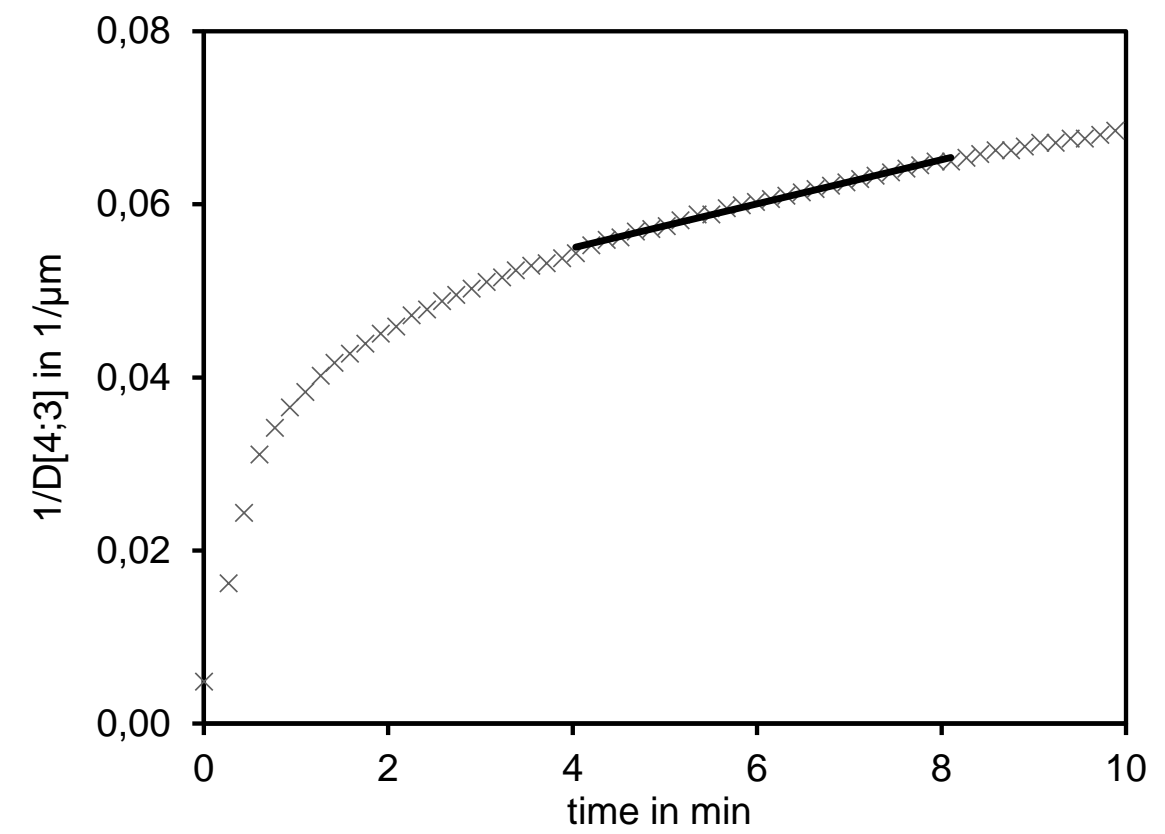

Figure 4.4.2: Evaluation of the desagglomeration rate a respectively slope between 4 and $8 \mathrm{~min}$

It can be seen that after a certain period of time an almost linear gradient occurs. As described in the patent [1] the slope between 4 and $8 \mathrm{~min}$ is determined by means of the following equation:

$$
y=m * x+c
$$


Where $y$ is the vertical change, $x$ is the horizontal change, $c$ is the vertical intercept (where the line theoretically crosses the Y-axis) and $m$ is the slope for the straight line. This value $(m)$ is referred to as the desagglomeration rate $\alpha$ which should be an indicator for the speed of the size reduction [1]. The steeper the slope, the easier it should be to reduce the particle sizes by means of ultrasonic treatment. Previous investigations [3] showed, that following parameters do influence the final outcome of the results while using this technique and evaluation:

- weight of sample respectively concentration

- $\quad$ setting of ultrasound (amplitude, pulsation and time)

- flow velocity of the suspension

- temperature of the system

The principle of measurement in accordance with the lasergranulometry respectively determination of the desagglomeration rate $\alpha$ has certain limitations. On the one hand, the comparable long measurement time $(10 \mathrm{~min})$ results in two negative side effects. The longer the ultrasonic sonotrode is turned on, especially at higher amplitudes, the faster it is worn and therefore has to be replaced. Additionally, the whole system heats up during the measurement and has therefore to be cooled down before continuing with a new sample. On the other hand, the evaluation of results only takes one parameter per measured curve into account, which is the volume weighted mean.

In the present chapter, a variety of different types of silica was investigated in accordance with the lasergranulometry. Furthermore, the measurement sequence as well as the evaluation of results were adjusted to reduce the mentioned disadvantages and to gain a deeper insight into the easiness and speed of cluster breakage. This new developed measurement technique is named in-situ cluster fragmentation.

\subsubsection{Theoretical Principles}

Silica samples circulate in a closed water loop including three main elements (Fig. 4.4.3). The first part (1) is the laser diffraction measurement system (Mastersizer 3000 by Malvern Instruments Limited) itself which measures the particle size distribution. It consists of two laser light sources, a He-Ne-laser respectively red laser (632.8 nm wavelength) and a LED light source or blue laser (470 nm wavelength) [2]. The second component (2) is the ultrasonic device (Branson Digital Sonifier 450D incl. a flat $1 / 2$ " tip by BRANSON Ultrasonic Coop.) which is attached to a cooled $\left(15^{\circ} \mathrm{C}\right)$ continuous flow cell (Sonifier Cell Disruptor by BRANSON UItrasonic Coop.). This cooling system is permanently turned on to prevent the system from heating up due to the heat transfer from the sonotrode. The last element (3) is a wet dispersion unit (Hydro SM by Malvern Instruments Limited) which ensures and controls the flow speed of the suspension.

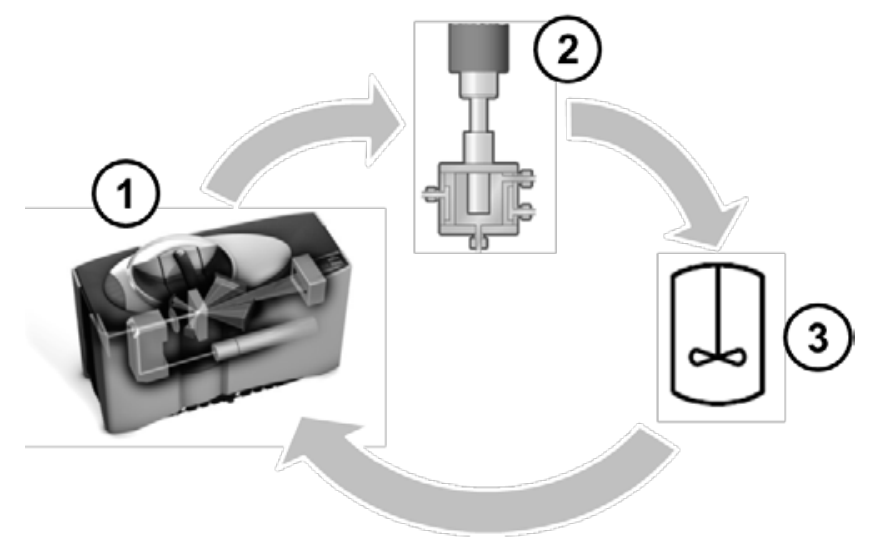

Figure 4.4.3: Schematic view of the measurement setup [2] 
With this setup it is possible to continuously measure the particle size distribution respectively reduction over a period of time while being treated with ultrasound. During the measurement, the laser beams pass the measurement cell with the flowing suspension of silica and water. When a beam hits a particle different interactions and optical effects occur which have to be taken into account [4]:

- Absorption is the reduction of the intensity of a light beam by particles.

- Scattering is defined as the change in propagation of a light beam at the interface of two media having different optical properties.

- Diffraction is the scattering of a light beam around the outline of a particle.

- Reflection is the change of the direction of a light beam at the surface of a particle without changing the wavelength or frequency.

- Refraction is a process where the direction of wave propagation changes by passing through a transmission medium.

- Extinction is the attenuation of a light beam throughout a medium by means of absorption and scattering.

- Transmission is the fraction of incident light that is not attenuated by the particles.

All these effects result in a scatter pattern which is recorded by a detector array surrounding the measurement cell in different directions (0.015 to 144 degree setup). In this way, also backscattering can be detected. The bigger the particle sizes, the smaller the deflection of the beam as shown in Fig. 4.4.4:
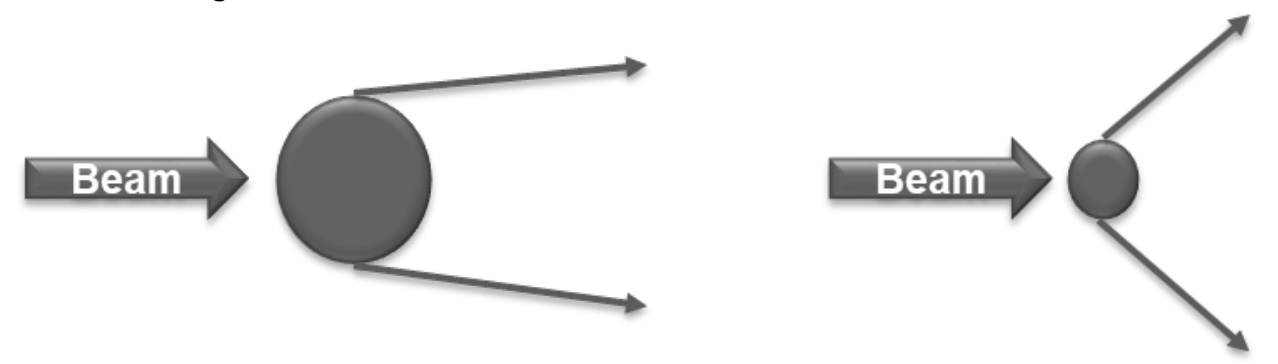

Figure 4.4.4: Scheme of the deflection of a beam in dependence of the particle size

Each specific diffraction pattern respectively scattering angle corresponds to a defined particle size in the range of $3000 \mu \mathrm{m}$ down to $10 \mathrm{~nm}$. In general, all light diffraction instruments are based on three basic assumptions [4-5]:

- All particles have a spherical shape.

- No multiple scattering occurs e.g. scattered light from one particle interacts with another particle.

- Each signal detected is a sum of the scattering patterns for one particular particle size. Additionally, each signal is generated by an incident beam exclusively interacting only once with a particle.

The systems includes a Fourier lens to ensure that all particles of an identical size are scattered to the same part of the detector array. By means of the obtained pattern the Mastersizer 3000 software calculates the volume based concentration of the sample by means of the Beer-Lambert law as expressed in equation 4.4.2:

$$
T=\frac{I}{I_{0}}=e^{-\propto_{i} * b}
$$

where $T$ is the relative transmission measured by the device, I represents the intensity of light at the distance $b$ in $\mathrm{mm}$ inside the particle field, $I_{0}$ is the initial intensity of the beam measured without a sample and $\alpha_{i}$ is the absorbance. 
The latter includes information about volume based particle sizes and concentration as following:

$$
\alpha_{i}=Q_{i} * \pi * r^{2}{ }_{i} * \boldsymbol{n}_{i}
$$

with $Q_{i}$ representing the efficiency of the extinction of light for a particle radius $r_{i}$ which can be calculated by different optical models described later in this chapter. The cross-sectional area of the particle is given by $\pi^{*} r_{i}^{2}$ and the total amount of particles is expressed by $n_{i}$. Taking the volume of particles $V_{i}$ into account, equation 4.4 .3 is adjusted for all particles inside the sample:

$$
\alpha_{i}=\frac{3}{4} \sum \frac{Q_{i} * V_{i}}{r_{i}}
$$

When now expressing the particle size by the diameter $d$ in $\mu \mathrm{m}$ and separating the volume term into a relative volume distribution $v$ and the total concentration $C_{v}$, the equation is described as following:

$$
\alpha_{i}=\frac{3}{2} C_{v} \sum \frac{Q_{i} * v_{i}}{r_{i}}
$$

When finally substituting eq. 4.4.5 into eq. 4.4.2 the volume concentration for each particle size can be calculated [2].

\section{Fraunhofer versus Mie Theory}

The particle size distribution is calculated by comparing the obtained diffraction pattern with different optical models. The most common ones are the Mie and the Fraunhofer scattering theory in accordance with ISO 13320 [4].

The Fraunhofer approximation is independent on material parameters and only takes simple diffraction effects into account. Depending on the literature, the smallest particles being detected by this theory possess a diameter of at least $2 \mu \mathrm{m}$ [5-6]. Not all effects of the light beam can be covered by this approximation below $1 \mu \mathrm{m}$ due to the fact that beside scattering also reflection, absorption and refraction occur. According to ISO13320 [4] the Fraunhofer theory is applicable for particle sizes of at least $50 \mu \mathrm{m}$, for smaller sizes the Mie theory offers the better solution. However, the use of the Mie theory requires additional parameters which are the refractive index of the liquid, the refractive index of the material and the imaginary refractive index, also referred to as the absorption index of the sample [6].

To check the influence of both theories on the final result, the following test was conducted. One type of silica (215GR210 - rubbed through a $500 \mu \mathrm{m}$ sieve) was prepared in three different suspensions. In each case $5 \mathrm{~g}$ were mixed with $50 \mathrm{ml}$ of demineralized water and prepared by means of an external ultrasonic device (Branson Digital Sonifier 450D incl. a flat $1 / 2$ " tip by BRANSON Ultrasonic Cooperation). In this way, it was possible to focus on the particle size measurement system by using the identical suspension prepared beforehand. The suspensions were once treated for 5, 10 and 15 min with an amplitude of $100 \%$. Subsequently, $5 \mathrm{ml}$ of each sample was fed to the laser diffraction system and the particle size distributions were calculated by means of the Fraunhofer and the Mie theory. During this measurement, the internal ultrasonic device was not used. For the use of the Mie approximation the density of silica was set to $2.0 \mathrm{~g} / \mathrm{cm}^{3}$, the refractive index of water was set to 1.33 , the refractive index of silica was set to 1.44 and the absorption index was set to 0.001 [7-8]. Fig. 4.4 .5 depicts the six measurements which were conducted. 

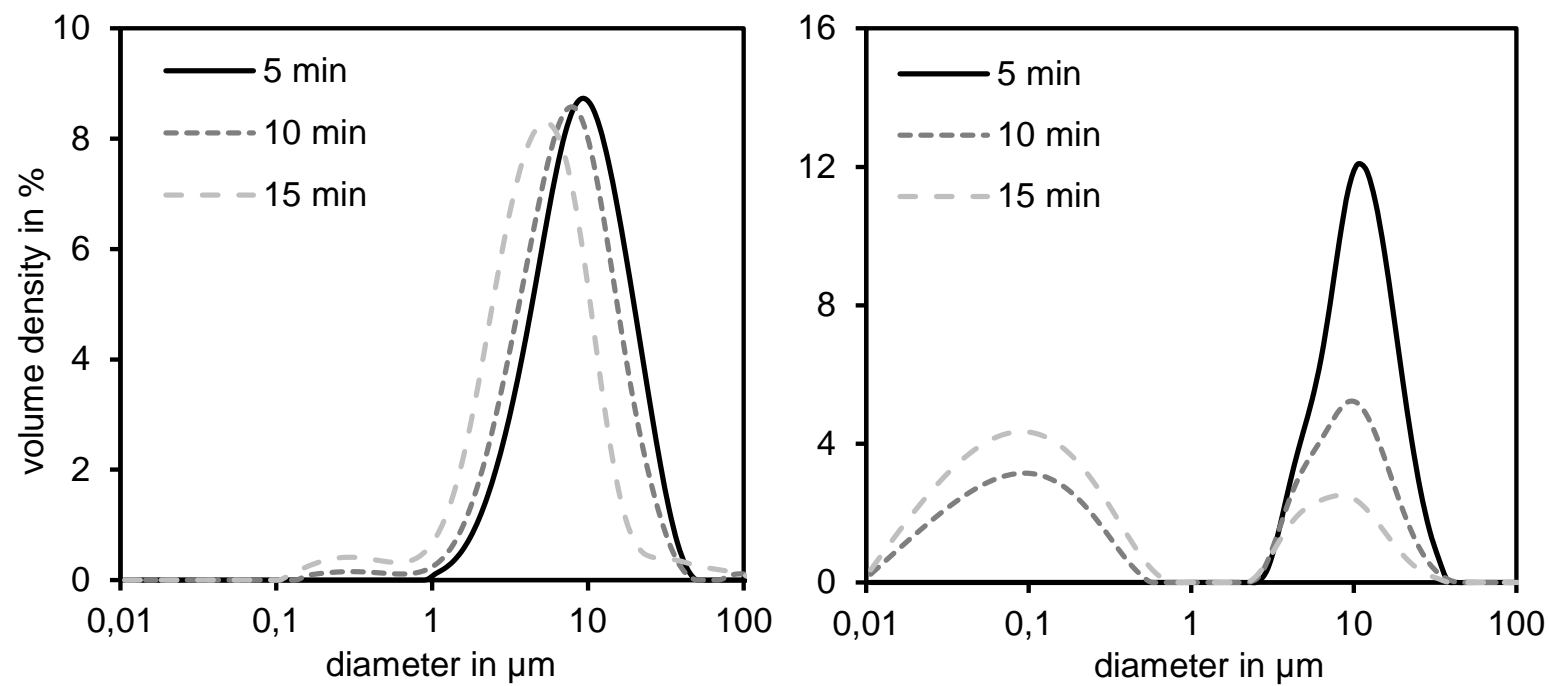

Figure 4.4.5: Example for calculated particle size distribution curves after different sonification times by means of the Fraunhofer (left) and Mie (right) theory

The picture on the left-hand side shows the volume density as a function of particle diameter calculated by the Fraunhofer approximation. It can be seen that a longer ultrasonic treatment respectively a higher energy input results in a shift of the mode (peak around $10 \mu \mathrm{m}$ ) to smaller particle sizes. In addition, a second peak shows up at a diameter of roughly $0.5 \mu \mathrm{m}$, which, in accordance with the theory, should not be possible to be detected.

In contrast to this, the plot on the right-hand side depicts the particle size distribution calculated with the Mie theory. The main peak is slightly shifted to bigger particles in comparison with the Fraunhofer theory. With increasing sonification time this peak is significantly reduced in its volume density whereas a second peak arises at $0.1 \mu \mathrm{m}$ after $10 \mathrm{~min}$. It is noticeable that the area in between both peaks (approx. $1.0 \mu \mathrm{m}$ ) possesses no particles at all which seems to be an effect due to the calculation. In addition, the result of the Mie theory is strongly dependent on the optical parameters chosen [5].

During the procedure of the lasergranulometry the ultrasonic device treats the suspension for 10 min. However, it is used in a pulsed mode which means it is turned on and off alternating for $1 \mathrm{~s}$ which results in an active time of $5 \mathrm{~min}$. Moreover, only approximately $1 / 3$ of the suspension is in direct contact with the sonotrode whereas $2 / 3$ are inside the tube system, stirring device and measurement cell. Therefore, the effective treatment corresponds roughly to $1 \mathrm{~min}$ and $40 \mathrm{~s}$. Comparing this treatment time with the experiment conducted above, where the shortest time was 5 min with $100 \%$ amplitude, it is obvious that almost no particles smaller than $1 \mu \mathrm{m}$ can be detected during the measurement. This can be explained by the fact that the laser diffraction system works on a volume based theory. As an example, one particle of a diameter of $100 \mu \mathrm{m}$ is taken into account. To achieve the same volume and hence the same peak height with particles of $1 \mu \mathrm{m}$ in diameter a total amount of one million samples is needed as depicted in Fig. 4.4.6. Therefore, the presence of several bigger clusters overlap most likely the signals of existing small particles.

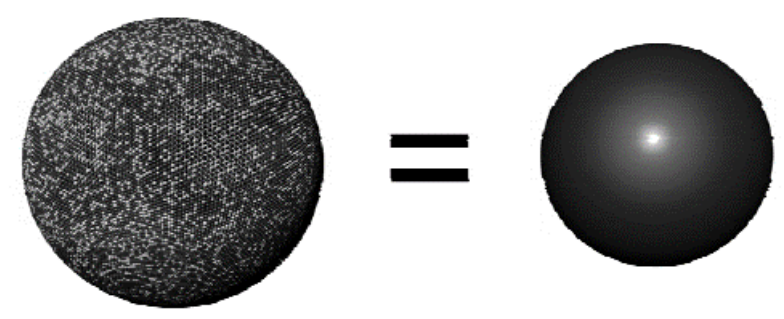

Figure 4.4.6: Schematic view of the volume of one particle (100 $\mu \mathrm{m}$ in diameter) equate to 1 million particles (1 $\mathrm{mm}$ in diameter) 
Beside all these points, the Fraunhofer and the Mie approximation seems to provide both results which are useable. However, both approaches include assumptions which contain errors. Due to the facts that the precise knowledge about the optical properties of silica is missing and the Fraunhofer theory was used for the lasergranulometry before it was decided to use this approximation having in mind the final results can only be compared relatively to each other.

\subsubsection{Method Development}

In order to develop a suitable method to investigate different types of silica the basic principles as described in chapter 4.1 have to be taken into account. In the present case, the foundations for upcoming tests are the lasergranulometry [1] and the investigations conducted by Jokiel [3]. Therefore, two different approaches were made. First of all, the default settings in accordance with the previous mentioned determination of the desagglomeration rate a were adjusted to overcome the relatively long measurement time and to preserve the sonotrode of fast wear. In a second step, different curve characteristics respectively evaluation possibilities beside the mean value were taken into account to gain more information about the easiness and speed of cluster breakage. For the latter it is necessary to be aware of the parameters which can be varied within the measurement. Finally, it has to be checked whether the adjusted method shows a sufficient reproducibility and if different storage conditions affect the final results. Following list summarizes the investigations which were conducted:

- determination of the desagglomeration rate $\alpha$

- reduction in measurement time

- evaluation possibilities

- variation of weight of sample

- variation of ultrasound (amplitude and pulsation)

- variation of flow velocity

- influence due to temperature fluctuation

- repeatability and reproducibility

- storage tests

In principle, it is also possible to vary the used liquid which has to inhibit the silica to re-agglomerate [9] and has to be inert to the device, tubes and the measurement cell. But demineralized water is still the easiest solution and is therefore applied for all following experiments. Silica powders and micro-pearls can directly be used for this measurement device whereas granules have to be pre-treated due to the fact that they are too large to pass the tube system. As a consequence, all granules are rubbed through a $500 \mu \mathrm{m}$ sieve by hand to reduce their size without changing the material's structure. 


\section{Adjustment to the Default Settings}

In case of the in-situ cluster fragmentation the default settings are referred to as the settings investigated by Jokiel [3] to replicate the lasergranulometry [1]. Following setups were therefore chosen as the standard measurement procedure:

- Weight of sample: $50 \mathrm{mg}$

- Analysis temperature: $20-25^{\circ} \mathrm{C}$

- Analysis liquid: demineralized water

- Ultrasonic treatment: $100 \%$ amplitude, pulsation (1 s on / 1 s off), 10 min

- Measurement time: $10 \mathrm{~min}$

- Stirring speed: $3000 \mathrm{rpm}$

- Evaluation: $\quad$ Rate $\alpha=$ slope $1 /$ mean between 4 and 8 min [1]

The test procedure was conducted as follows: $150 \mathrm{ml}$ of demineralized water was filled into the device. While the liquid circulated throughout the system the temperature was kept between 20 and $25^{\circ} \mathrm{C}$ by means of the cooled $\left(15^{\circ} \mathrm{C}\right)$ continuous flow cell. Subsequently, $50 \mathrm{mg}$ of the sample was added to the liquid through the opening of the dispersion unit and the test procedure can be started. The Fraunhofer approximation was used for all experiments in this chapter to calculate the volume size distribution. In total 60 curves are recorded per test and each single measurement took $5 \mathrm{~s}$ to obtain a statistically relevant quantity of particles for the evaluation. In between each measurement, a $5 \mathrm{~s}$ break was conducted. Therefore, a new curve was generated every $10 \mathrm{~s}$. First, three different types of silica (161GR193, 165P196 and 157MP207) were investigated by means of the lasergranulometry and the desagglomeration rate a was determined. To check if the measurement time can be reduced these examples (inverse mean over time) were firstly plotted (Fig. 4.4.7) as follows:

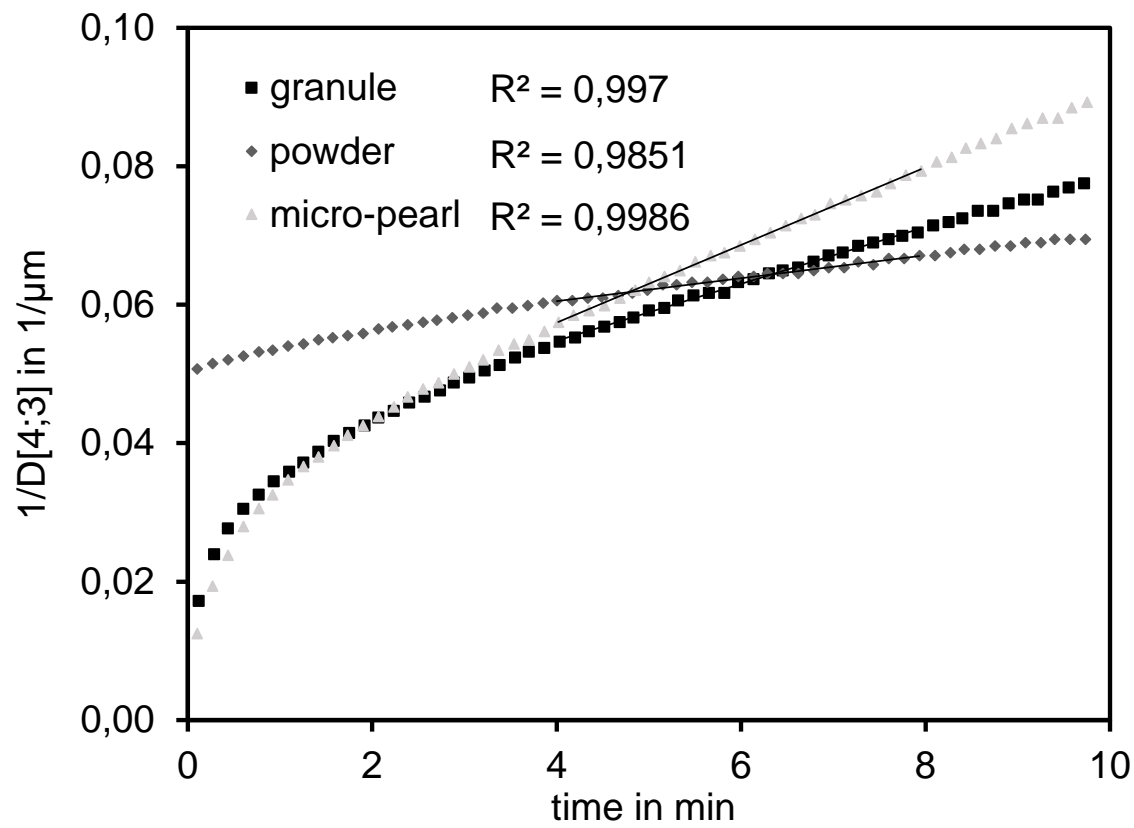

Figure 4.4.7: Evaluation of the desagglomeration rate $\alpha$ for one silica granule, powder and micro-pearl

It can be seen that the coarser dosage form of granules and micro-pearls show a non-linear slope in the first $1 \frac{1 / 2}{2}$ min whereas powders exhibit an almost steady gradient throughout the measurement. The whole measurement took $10 \mathrm{~min}$ and the slope between 4 and 8 min was recorded. Two aspects are noticeable. First of all, it seems to be unnecessary to run the measurement for $10 \mathrm{~min}$ due to the fact that the evaluation is completed after $8 \mathrm{~min}$. 
Secondly, a decent linearity is already reached after 2 min of ultrasonic treatment. Therefore, following adjustments were made:

- Reduce measurement time to 6 min

- Evaluate results between 2 and 6 min

All other parameters of the measurement procedure were kept constant. Fig. 4.4 .8 depicts the same silica samples as tested beforehand, only measured and evaluated with the two adjustments mentioned above. Additionally, the X-axis was changed to seconds (s) instead of minutes ( $\mathrm{min}$ ) in accordance with ISO 80000-1 [10] respectively the international system of units.

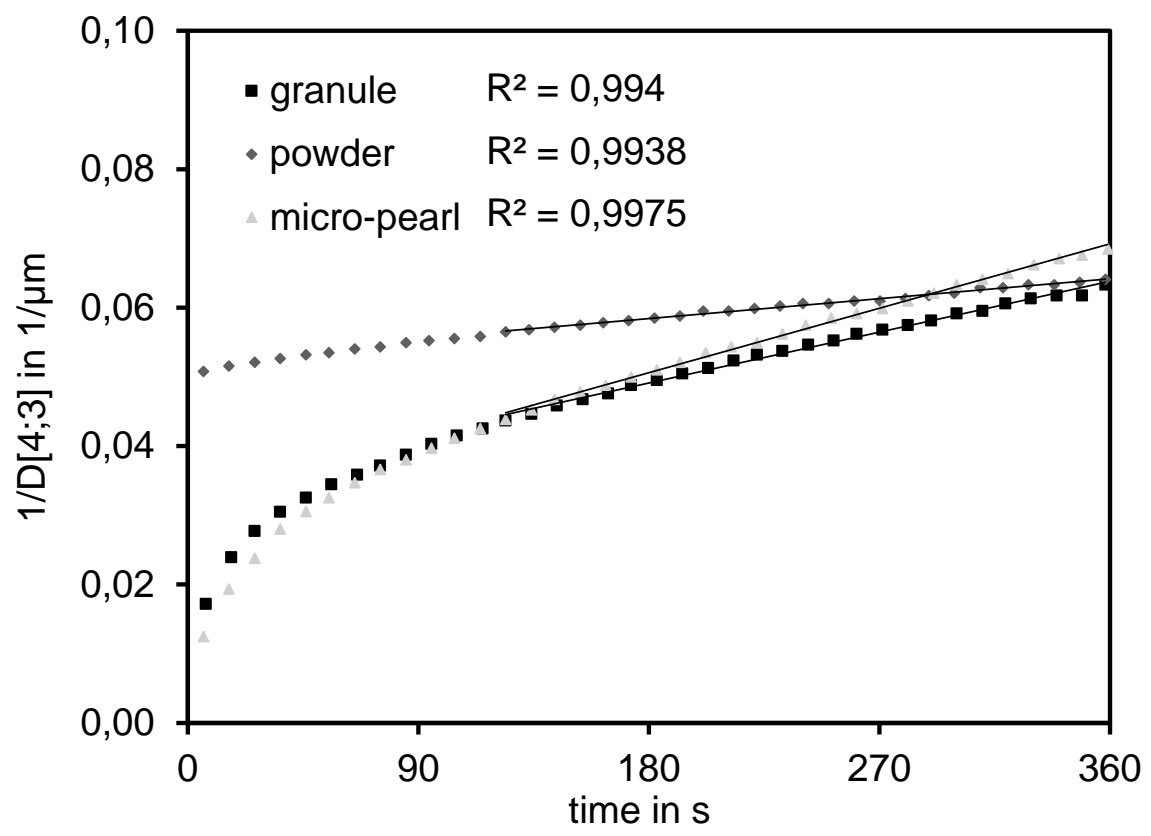

Figure 4.4.8: Evaluation of the mean value for one silica granule, powder and micro-pearl

It is shown that the linearity (fitted regression line $\mathrm{R}^{2}$ ) within the new evaluation range is as good as for the standard measurement procedure. The adjustment of X-axis unit (min to s) leads to comparable small calculated slopes (numerical values) which only can be differed from the fifth decimal on. Therefore, all results were multiplied by $10^{4}$ to deal with more manageable numbers. This adjustment does not affect the final outcome and was only made due to practical reasons.

Finally, 25 types of silica with different dosage forms were tested with the standard procedure and the adjusted measurement (each measurement was repeated 3 times). Fig. 4.4.9 depicts the correlation between the desagglomeration rate $\alpha$ (standard procedure) and the new evaluation $\left(1 / \mathrm{D}[4 ; 3](2-6 \mathrm{~min}) * 10^{4}\right)$. 


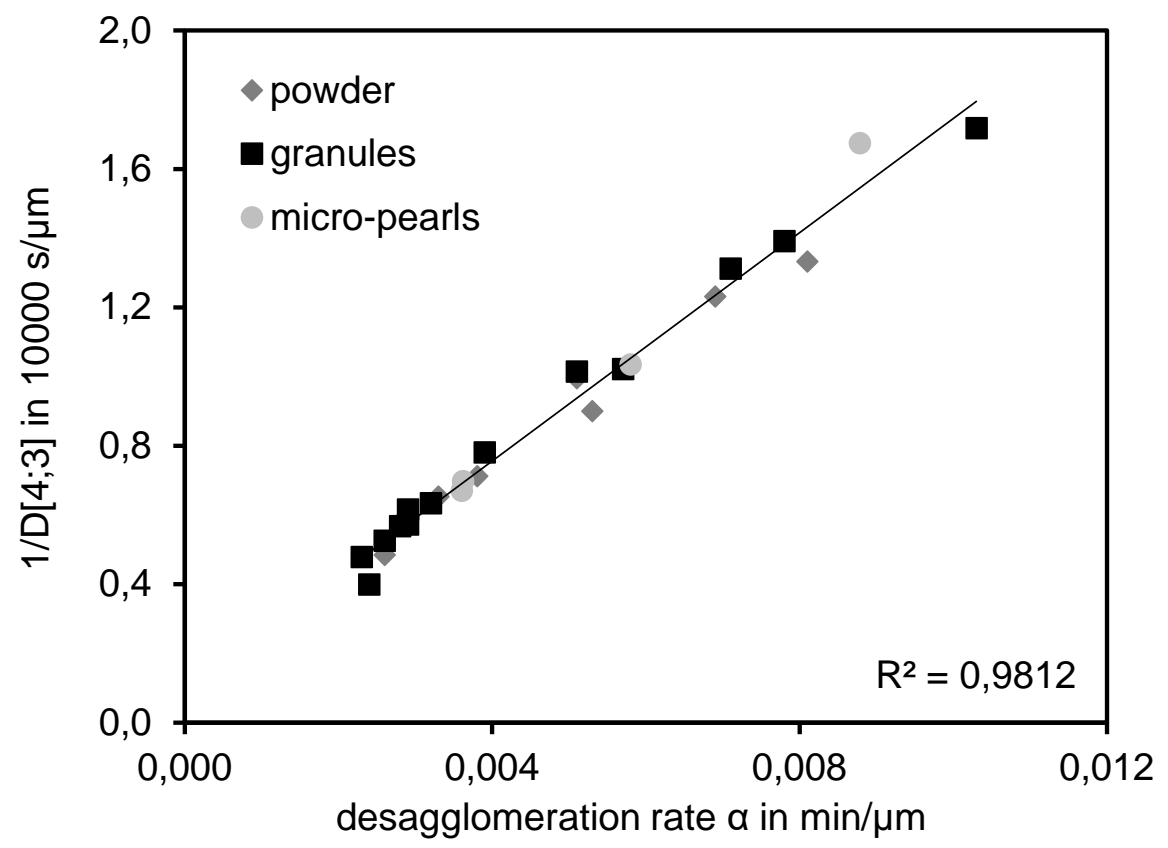

Figure 4.4.9: Correlation between the desagglomeration rate $\alpha$ and the mean degradation rate

It can be seen that a very high linear correlation between both methods is obtained. With shortened measurement time a prevention of a too fast wear of the sonotrode can be achieved. Additionally, the heating of the system is reduced. Therefore, influences due to different temperatures can be avoided. As a result, this new test procedure is used for all upcoming measurements. To emphasize the change of the method respectively evaluation, this technique is from now on referred to the in-situ cluster fragmentation (ICF). In the next steps, it was tested whether it is possible to evaluate additional values of the curve progression.

\section{Evaluation possibilities}

To follow the idea of observing the particle size reduction of a silica sample inside a watery suspension, different characteristic values of the distribution curve can be monitored. These values have to fulfill certain requirements. First of all, the results have to be highly repeatable with a comparable low standard deviation. Secondly, it should be possible to distinguish between different types of silica and finally, a linear shift should occur to evaluate the data between 2 and 6 min. All following values were investigated for the mentioned 25 different silica grades and each type was tested three times. The most common curve characteristics are depicted in Fig. 4.4.10.

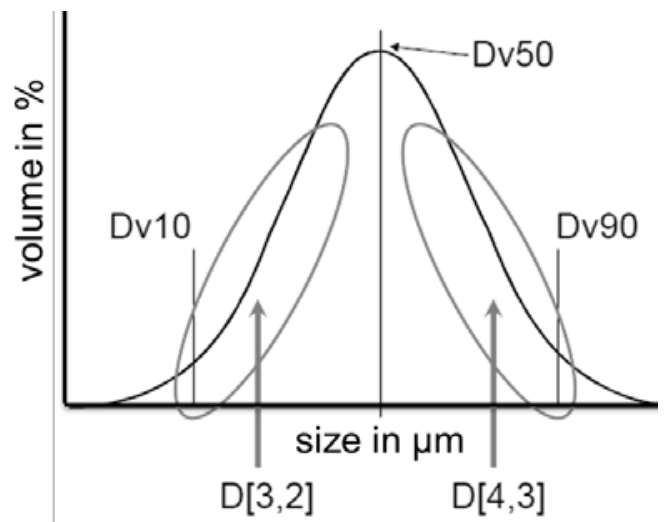

Figure 4.4.10: Characteristic values of a particle size distribution curve

The value Dv50 corresponds to the medium particle size in $\mu \mathrm{m}$ where 50 vol\% of the sample is smaller and $50 \mathrm{vol} \%$ of the sample is larger than the given diameter (percentage by volume). 
It is also known as the median of the volume distribution. Likewise, the Dv10 value corresponds to the size where $10 \mathrm{vol} \%$ of the sample are smaller and for the Dv90 value $90 \mathrm{vol} \%$ of the samples are smaller than the given diameter. Fig. 4.4.11 depicts the evaluation of the three DV values for one type of silica 159GR204.

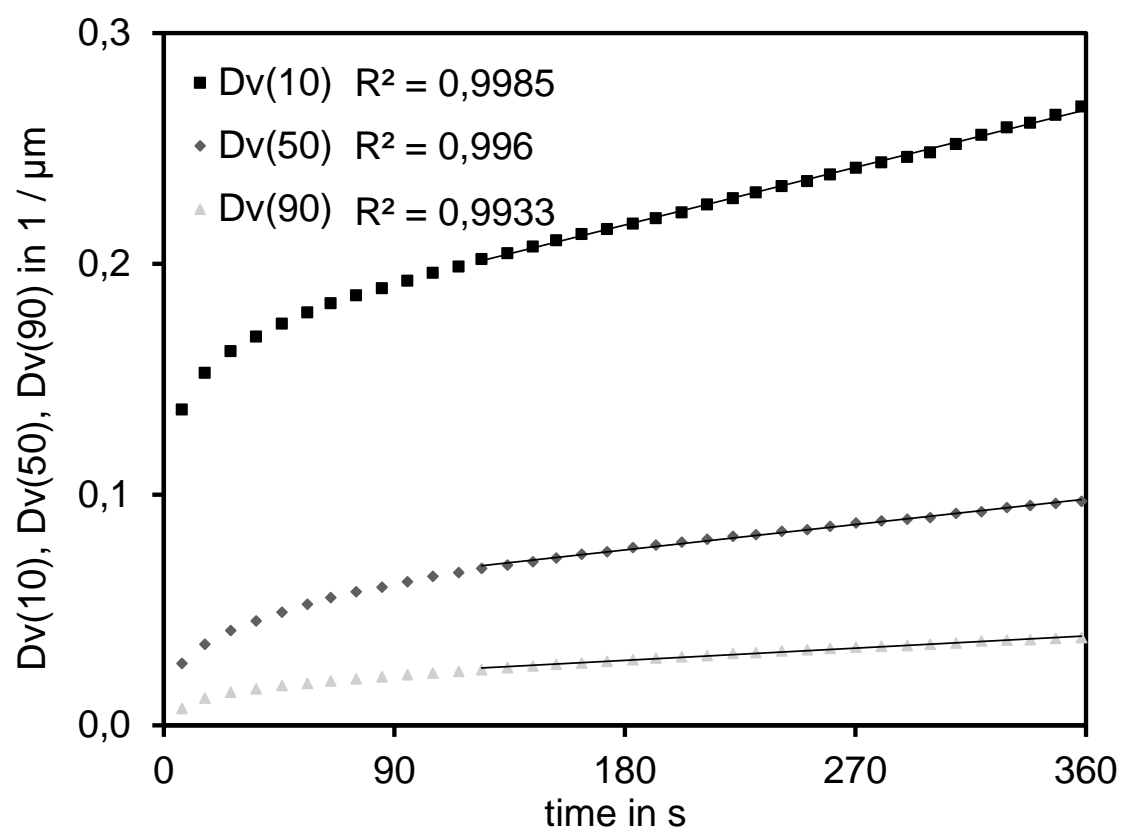

Figure 4.4.11: Evaluation of the $\operatorname{Dv}(10), \operatorname{Dv}(50)$ and $D v(90)$ value

It can be seen that all three values show a high linearity within the evaluation range. This is also valid for every other tested silica, therefore it can be said that the $\operatorname{Dv}(10), \operatorname{Dv}(50)$ and Dv(90) are suitable parameters to evaluate the results of the ICF technique.

The $D[4 ; 3]$ value correspond to the volume weighted mean or De Brouckere mean, often simply called "mean" and is expressed as follows [11]:

$$
D[4 ; 3]=\frac{\sum_{1}^{n} D_{i v i}^{4}}{\sum_{1}^{n} D_{i v i}^{3}}
$$

With $D_{\text {ivi }}$ is the size for each measured diameter with a defined volume. As shown in previous examples (e.g. Figure 4.4.9) the mean value shows a high linearity and are therefore applicable to use. All 25 tested silica samples can be evaluated in this way. From now on, this evaluation of the mean value is named the mean degradation rate.

Similar to the mean, the $\mathbf{D}[3 ; 2]$ value is the surface weighted mean or Sauter mean which describes the surface area distribution [11]:

$$
D[3 ; 2]=\frac{\sum_{1}^{n} D_{i v i}^{3}}{\sum_{1}^{n} D_{i v i}^{2}}
$$

The Sauter mean thereby is more sensitive to smaller particles and shows a linear correlation to the $\operatorname{Dv}(10)$ value $\left(R^{2}=0.998\right)$ as depicted in Fig. 4.4.12. This value is as well suitable to evaluate all tested silica grades. 


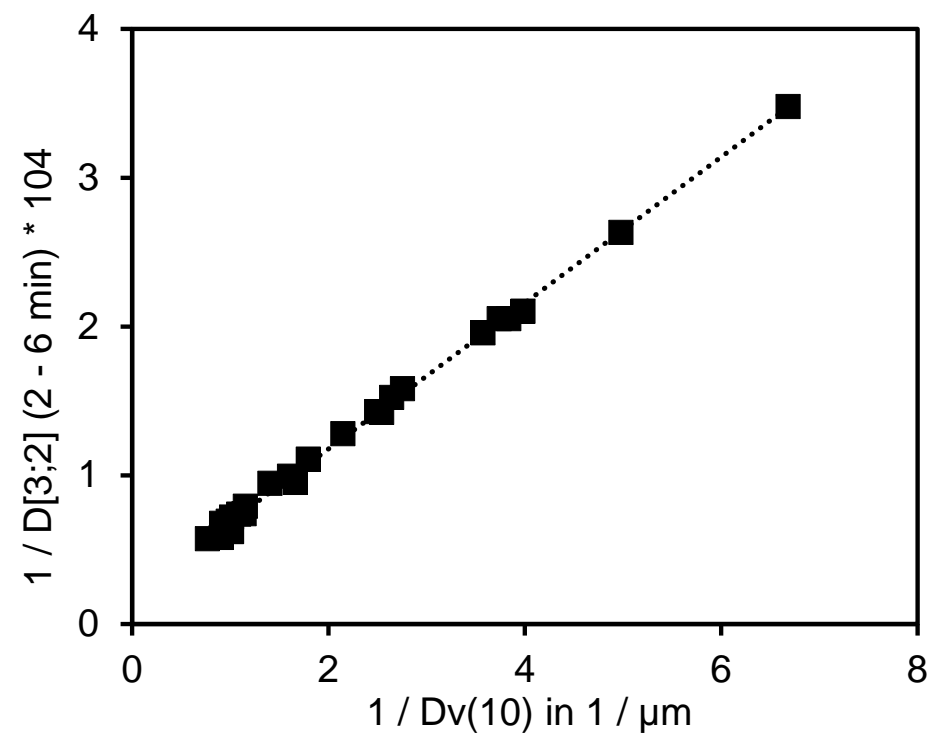

Figure 4.4.12: Correlation between the $D[3 ; 2]$ the $D v(10)$ degradation rates

The span corresponds to the width of the distribution and is calculated as following [2]:

$$
\operatorname{span}=\frac{D v 90-D v 10}{D v 50}
$$

It is expected that the width of the distribution is high at the beginning of the measurement and gets narrower until the end. This can be explained by the fact that bigger clusters are easily reduced in their size during the ultrasonic treatment whereas the Fraunhofer approximation limits particles smaller than $1 \mu \mathrm{m}$ to be detected. As a result, most types of silica show a reduction in the span over time. However, some grades e.g. 175GR203 show a different behavior as shown in Fig. 4.4.13:
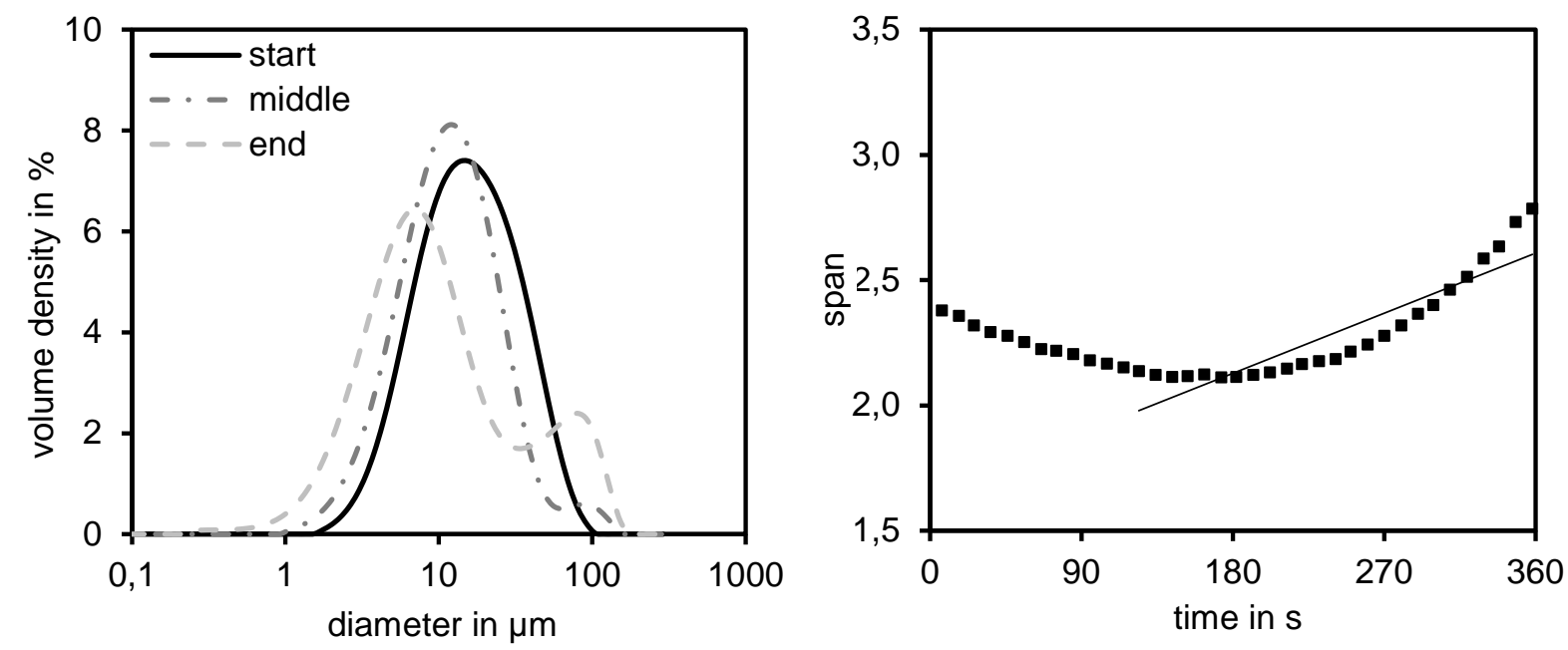

Figure 4.4.13: Calculated particle size distribution curves at the start, in the middle and at the end of sonification (left) and evaluation of its span values (right)

The picture on the left-hand side shows the volume density as a function of the particle diameter. During the measurement, the distribution curve shifts to smaller particle sizes as expected. However, a second peak at bigger diameters appears and grows over time.

This phenomenon results in a decrease of the span at the beginning of the measurement followed by a nonlinear increase as can be seen in the plot on the right-hand side. Therefore, it is not possible to evaluate a linear gradient of the span. 
The mode is the particle diameter in $\mu \mathrm{m}$ of the peak maximum of the volume size distribution curve. In case of a multimodal distribution e.g. for 161GR193 with several peaks the mode always corresponds to the largest peak. This fact leads to a certain risk as can be seen in Fig. 4.4.14.
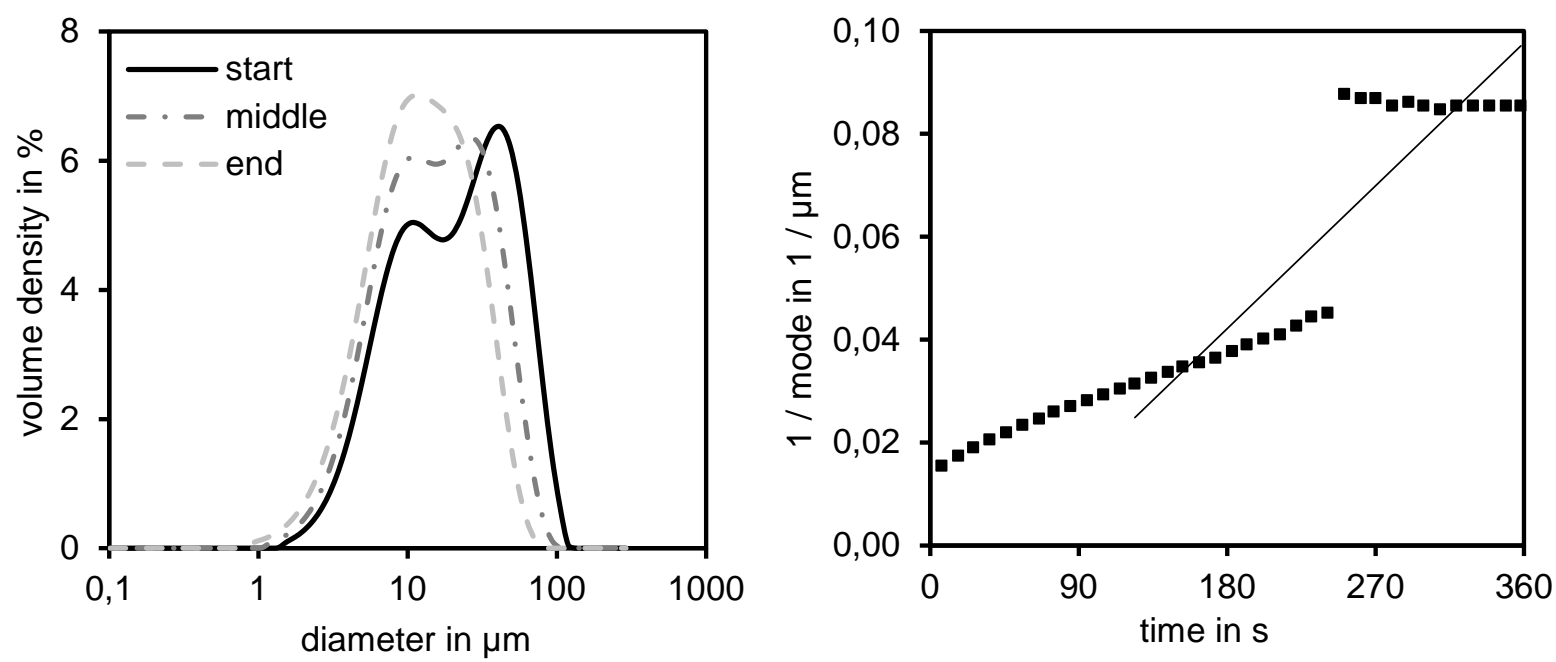

Figure 4.4.14: Calculated particle size distribution curves at the start, in the middle and at the end of sonification (left) and evaluation of its mode values (right)

The plot on the left-hand side demonstrates the volume density of one type of silica as a function of diameter for different times. At the start of the measurement, the curve possesses a bimodal distribution where the peak maximum occurs at larger diameters (peak 1). During the measurement (middle) this peak decreases whereas the smaller peak at lower diameters (peak 2) increases and at a certain point peak 2 turns into the maximum. This leads to a sudden change in the mode value as can be seen in the plot on the right-hand side. Therefore, it is not possible to evaluate a linear slope by following the change of the mode. This phenomenon is dependent on the type of silica and cannot be observed for all grades.

\section{Variation of parameters and their influence on the final results}

As a basis for all upcoming results the default settings are chosen and the new test procedure with adjusted time and evaluation between 2 and 6 min is used. Step by step, the sample weight, the ultrasonic treatment, the flow velocity and the temperature of the suspension are varied to check, how and whether they influence the final result. The main parameter which is investigated is the mean degradation rate $(1 / D[4 ; 3])$. However, the $D[3 ; 2], D v(10), D v(50)$ and Dv(90) are evaluated as well just in case that certain parameter variations only influence specific curve characteristics. These values are only presented in the following if some differences to the mean degradation rate can be seen.

\section{Sample weight}

The first varied parameter was the concentration of the sample. Therefore, one type of silica granule (215GR210) was firstly rubbed through a $500 \mu \mathrm{m}$ sieve and subsequently tested in three different amounts where $50 \mathrm{mg}$ are referred to as the default mass. Moreover, the quantity was reduced to $20 \mathrm{mg}$ and increased to $80 \mathrm{mg}$. Each measurement was conducted three times. Fig. 4.4.15 depicts the effect of different sample concentrations on the mean degradation rate as well as on the $\mathrm{Dv}(50)$ value which shows a different behavior. 


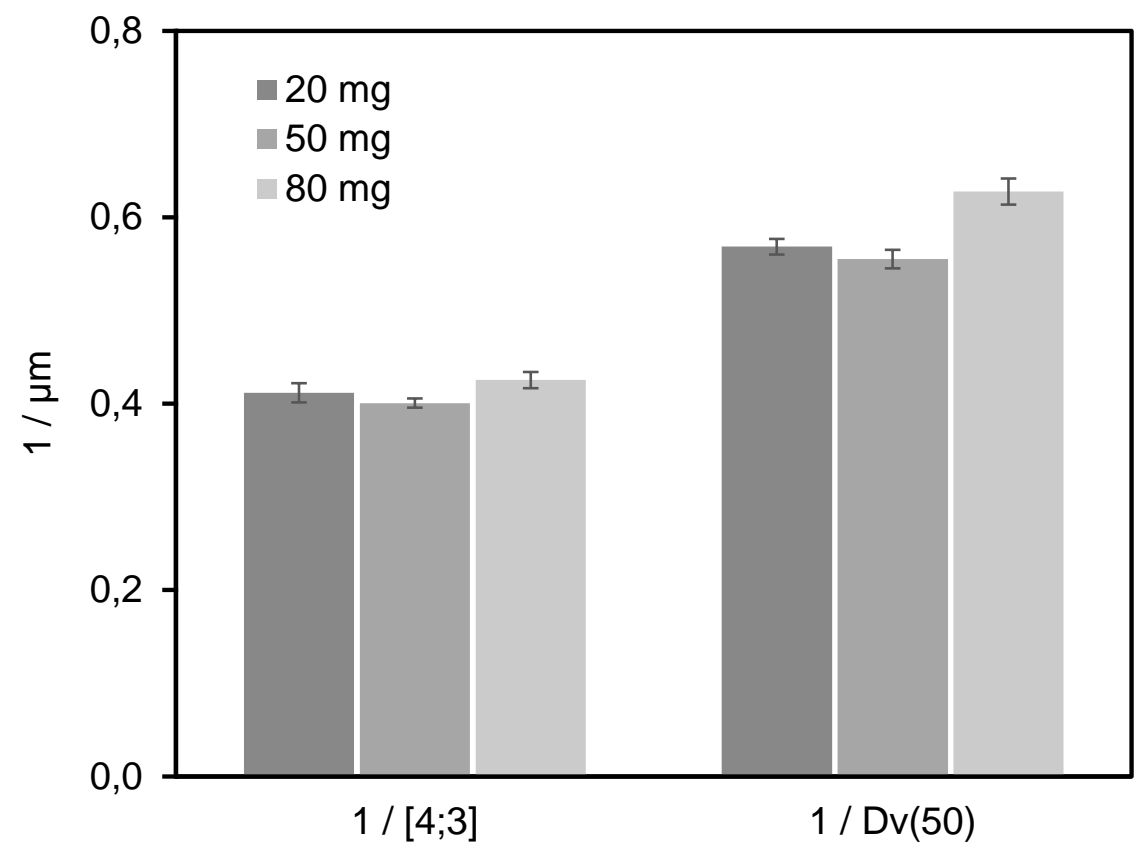

Figure 4.4.15: Influence of different sample weights on the evaluated mean degradation rate and $\mathrm{Dv}(50)$ values

First of all, it can be seen that the variation of the sample quantity hardly affects the mean degradation rate including a high repeatability of results. The $\operatorname{Dv}(50)$ value however seems to be affected by using a higher mass $(80 \mathrm{mg})$. One explanation can be that a higher concentration results in a higher chance of collision of different particles inside the suspension which leads, beside the cavitation itself, to an additional reduction in size. The laser obscuration can influence the final result as well. It is defined as the reduction of the initial laser beam intensity throughout the liquid without any sample. It is recommended [2] to work with a laser obscuration of 10 to $20 \%$ in order to get reliable results. With $20 \mathrm{mg}$, an obscuration of approximately 2 to $6 \%$ could be achieved. $80 \mathrm{mg}$ lead to a level of 8 to $23 \%$ whereas $50 \mathrm{mg}$ were in the range of 5 to $14 \%$. Therefore, $\mathbf{5 0} \mathbf{~} \mathbf{m g}$ of silica are chosen as a final setting for all following experiments.

\section{Variation of ultrasonic treatment}

The speed of particle size reduction is mainly dependent on the energy input by means of the ultrasonic treatment. This can be controlled by two factors, the amplitude and the sonification time. The total measurement time was already adjusted and shortened for the evaluation. The reduction from 10 to 6 min contains an incubation time of 2 min to be independent of the dosage form followed by 4 min of measuring the size reduction. However, the ultrasonic treatment time can be shortened additionally by using the ultrasound in a pulsed way. This means that it is not permanently running but turned on and off alternately. This procedure is already used within the default setting where the amplitude was set to $100 \%$ and used in a pulsed way (1 s on / $1 \mathrm{~s}$ off). In theory, this energy input should directly correspond to half of the amplitude (50\%) but with permanent sonification (double effective treatment time). Therefore, as a first comparison four different types of silica containing two granules and two powders were tested with $100 \%$ - 1s on / 1s off - and $50 \%$ without pulsation. In addition, the amplitude was further decreased to 30 and $10 \%$ without using the pulsation possibility. Fig 4.4.16 depicts the effect of different ultrasonic settings on the mean degradation rate. Each experiment was repeated three times. 


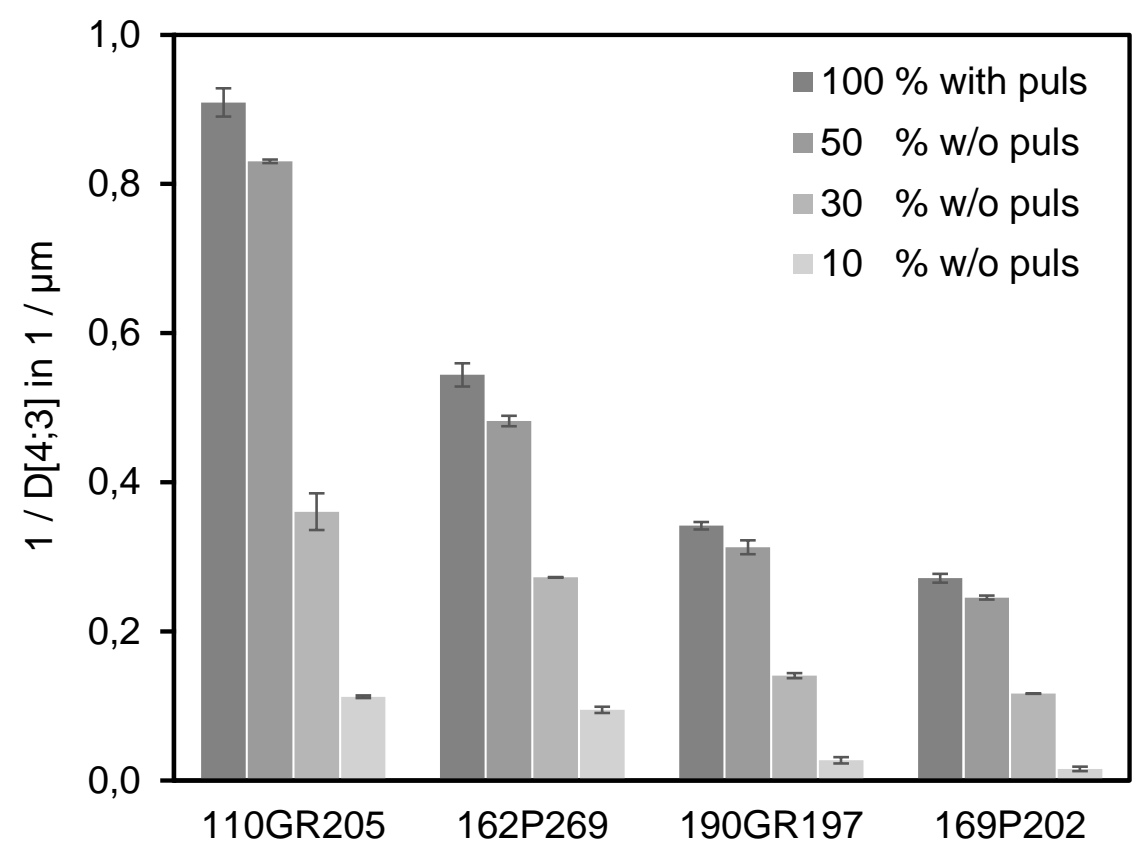

Figure 4.4.16: Effect of different ultrasonic treatment settings on the mean degradation rate

It can be seen that an amplitude of $100 \%$ including pulsation shows a slightly faster size reduction than an amplitude of $50 \%$ without (w/o) pulsation. One explanation can be that the higher amplitude causes a higher acceleration of particles resulting in heavier collision and therefore smaller sizes. Another possibility can be that the lower energy input due to $50 \%$ amplitude is not high enough to reduce the size of certain clusters independently of the treatment time. Reducing the amplitude to $30 \%$ without pulsation seems to be suitable as well, however, it becomes more difficult to distinguish between different types of silica. The relatively low values using permanently $10 \%$ of amplitude indicates that hardly any size reduction took place. Therefore, it is not possible to use this "10\% of amplitude" setting.

One unpredictable phenomenon appeared randomly while using ultrasound in a permanent state (50\% amplitude w/o pulsation). A new peak built up at large diameters (> $1000 \mu \mathrm{m})$ which can most likely be related to air inclusions being degassed from the liquid and measured by laser diffraction device. Although they show up in a much higher size range than the actual sample clusters $(<350 \mu \mathrm{m})$ it cannot be ruled out that the measurement might be influenced. Hence, it was decided to use $\mathbf{1 0 0} \%$ of amplitude including pulsation (1 s on I 1 s off).

The energy input in general is comparable low in comparison to other techniques using ultrasound to treat a silica suspension. The sedimentation method e.g. described in chapter 4.3 uses an amplitude of $70 \%$ for $4 \mathrm{~min}$. The CPS (disc centrifuge) [12] treats the suspension for 15 min with an amplitude of $100 \%$. In case of the ICF the suspension is treated 6 min with $100 \%$. However due to the pulsation the actual treatment time is only $3 \mathrm{~min}$. In addition, only roughly $1 / 3$ of the suspension is in contact with the sonotrode at every time of the measurement whereas $2 / 3$ of the suspension are located inside the tubes, the stirring device and the measurement cell. As a result, the effective treatment is $1 \mathrm{~min}$ at an amplitude of $100 \%$. Therefore, a further reduction of the energy input by decreasing the amplitude is inappropriate. 


\section{Variation of flow velocity speed}

Another element of the measurement setup is the wet dispersion unit which ensures and controls the flow speed of the suspension. In order to investigate the influence of different stirring speeds on the mean degradation rate and Dv(50) value, one silica (162P269) was selected. For this, the revolutions per minute (rpm) were varied in 500 rpm steps from 1500 to $3500 \mathrm{rpm}$ in the following experiments as depicted in Fig. 4.4.17. Each condition was measured three times.

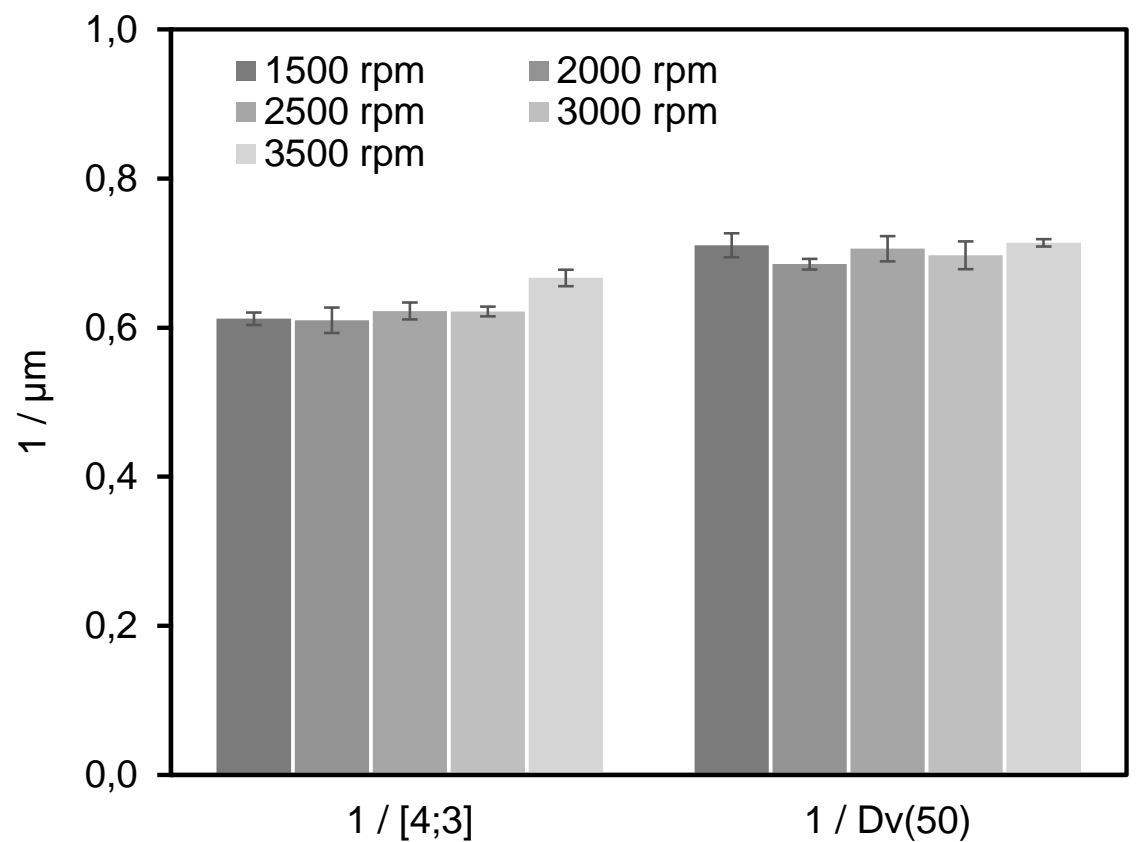

Figure 4.4.17: Influence of different velocity speeds on the evaluated mean degradation rate and $D v(50)$ values

It can be seen that the mean degradation rate is slightly increased at the highest velocity speed of $3500 \mathrm{rpm}$. However, overall, there is hardly any effect visible on both shown evaluation parameters without any possibility to rate the different settings. In general, it is expected that a higher velocity speed prevents particles from settling inside the system and increases the number of particles passing the laser device per measurement. Jokiel [3] mentioned a certain risk of trapping air inside the system while using $3500 \mathrm{rpm}$. Although this phenomenon could not be confirmed by present results, it was decided to maintain the velocity speed of $\mathbf{3 0 0 0} \mathbf{~ r p m}$ in accordance with the default settings.

\section{Temperature fluctuation}

During one measurement it could be observed that the temperature was slightly risen over time $\left(\max .+5{ }^{\circ} \mathrm{C}\right.$ ). This phenomenon was probably caused by cavitation and moreover by heat transfer from the vibrating sonotrode to the suspension as described in chapter 4.1. Without any cooling of the system while frequently being used, the start temperature of different measurements can vary. Therefore, a continuous flow cell is included to the ultrasonic device which permanently cools it down. In this way, it is possible to keep the starting temperature of each single measurement between 20 to $25^{\circ} \mathrm{C}$. To examine if the cooling influences the actual measurement, one silica (215GR210) was tested three times in a cooled (at $22^{\circ} \mathrm{C}$ ) and in an uncooled running system at $27^{\circ} \mathrm{C}$ starting temperature. The mean degradation rate as well as the $\operatorname{Dv}(50)$ value were evaluated as shown in Fig. 4.4.18: 


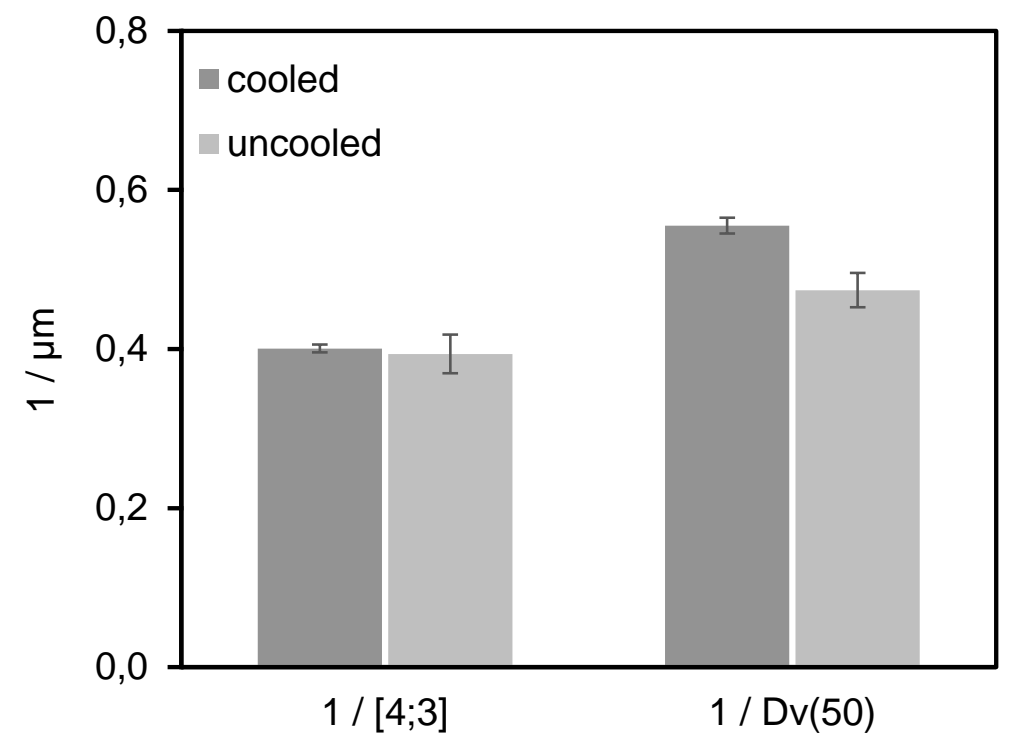

Figure 4.4.18: Influence of cooling on the evaluated mean degradation rate and Dv(50) values

As can be seen the mean degradation rate is not influenced by the temperature, however, the standard deviation is higher for the uncooled system. On the contrary, the Dv(50) is affected clearly in both, absolute value and standard deviation, at different temperatures. Therefore, it is recommended to use the continuous flow cell during the measurement to prevent irregularities in the final outcome.

\section{Repeatability and Reproducibility}

The investigations about the speed and easiness of the silica cluster breakage result in the following final settings and parameters for the in-situ cluster fragmentation method:

- Weight of sample: $\quad 50 \mathrm{mg}$

- Analysis temperature: $20-25^{\circ} \mathrm{C}$

- Analysis liquid: demineralized water

- Ultrasonic treatment: $100 \%$ amplitude, pulsation (1 s on / 1 s off), 6 min

- Stirring speed: $\quad 3000 \mathrm{rpm}$

- Theory:

- Evaluation:

Fraunhofer approximation

slope between 2 and 6 min for the inverse parameters of $\operatorname{Dv}(10), \operatorname{Dv}(50), \operatorname{Dv}(90), D[3 ; 2]$ and $D[4 ; 3]$

To examine the repeatability and reproducibility of this measurement and to check if it is possible to distinguish between different types of silica, 5 grades (two types of powders, granules and one micro-pearl) with a variety of analytical parameters were investigated. Three different operators tested each silica five times which results in 15 measurements per type of silica. Based on this, the standard deviation was determined. Fig. 4.4.19 depicts the results on the mean degradation rate. 


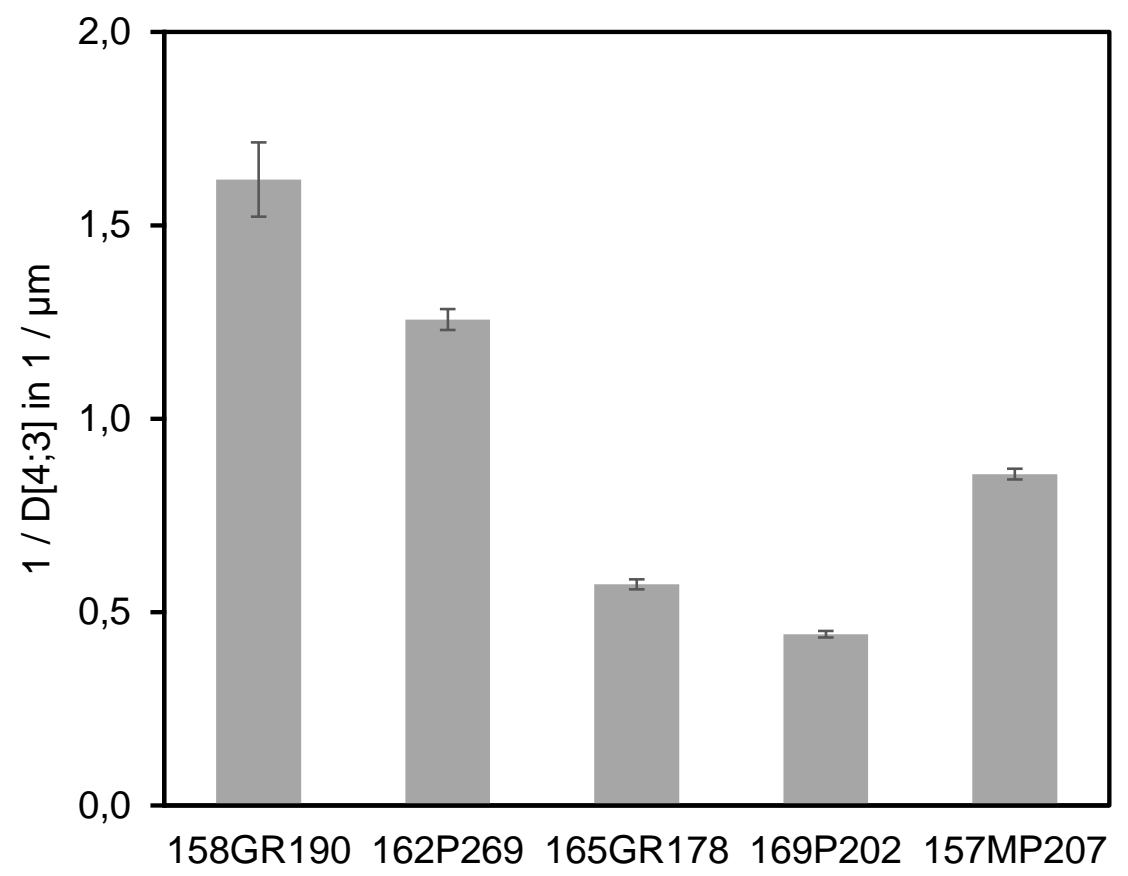

Figure 4.4.19: Reproducibility test of five different types of silica conducted by three operators (in total 15 measurements per grade)

It can be seen that all five silica grades can be sufficiently differed from each other and possess a relatively low standard deviation. This is as well true for the evaluation of the $\operatorname{Dv}(10), \operatorname{Dv}(50)$, $\mathrm{Dv}(90)$ and $\mathrm{D}[3 ; 2]$. A tendency is recognizable that the higher the mean degradation rate, respectively the easier it is to reduce the cluster size of silica, the greater the fluctuation of results. Overall, the in-situ cluster fragmentation method can be referred to be a suitable method in accordance with the basic requirements for an analytical method as described in chapter 4.1.

\section{Storage effect}

Before the modification of the original lasergranulometry method was performed, a long term storage test was conducted in accordance with the evaluation of the desagglomeration rate $\alpha$ [1]. One type of fresh produced silica powder (173P223) was therefore stored under three different conditions, at room temperature (RT), inside an oven at $50{ }^{\circ} \mathrm{C}$ and inside a freezer at $-18{ }^{\circ} \mathrm{C}$. The silica was firstly measured freshly, subsequently after one day and then on a weekly base. After 4 months, the frequency was reduced to one test per month (Fig. 4.4.20). 


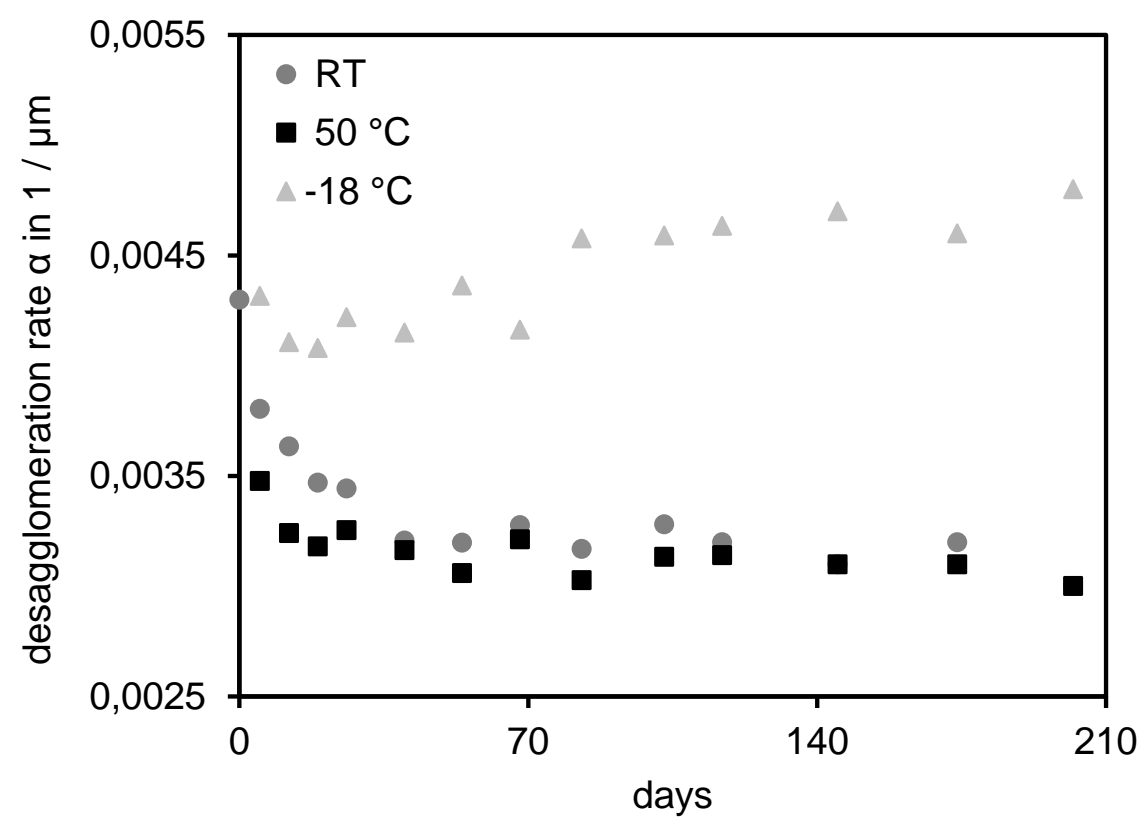

Figure 4.4.20: Artificial storage test under different conditions of one type of silica stored

The freshly produced silica possesses a desagglomeration rate $\alpha$ of $0.00431 / \mu \mathrm{m}$. When stored at room temperature (RT), $\alpha$ strongly decreases in the beginning of the study and slowly converges to a value of $0.0032 \pm 0.00011 / \mu \mathrm{m}$. Storing the sample inside an oven at $50{ }^{\circ} \mathrm{C}$ enhances this effect. After roughly one month both conditions end up at the same value which indicates that the silica has reached a limit where this analytical parameter does not change anymore. If, however, silica is stored inside a freezer at $-18^{\circ} \mathrm{C}$ this "aging" effect can be prevented. Even more, $\alpha$ seems to increase slightly over time.

To investigate the effect of storage time and conditions on the newly adjusted method, a final storage experiment was conducted, following the final settings. To carry this out, a freshly produced silica granule (168GR180) was tested and artificially aged under the following conditions (Tab. 4.4.1):

\section{Table 4.4.1: Conditions of the storage test for the in-situ cluster fragmentation}

\begin{tabular}{cc} 
Condition & Time \\
\hline Freshly produced & $0 \mathrm{~h}$ \\
$105^{\circ} \mathrm{C}$ (oven) & $24 \mathrm{~h}$ \\
\hline room temperature (RT) & 1 week \\
$50^{\circ} \mathrm{C}$ (oven) & 1 week \\
$-18^{\circ} \mathrm{C}$ (freezer) & 1 week \\
\hline $\mathrm{RT}$ & 1 month \\
$50^{\circ} \mathrm{C}$ (oven) & 1 month \\
$-18^{\circ} \mathrm{C}$ (freezer) & 1 month \\
\hline
\end{tabular}

All samples were stored in their original dosage form (GR) and crushed just before the sample was pre-treated via ultrasound. Each experiment was repeated three times and the standard deviation was determined. Fig. 4.4.21 depicts the results of the mean degradation rate of a freshly produced silica, after one week and after one month being stored under different conditions. 


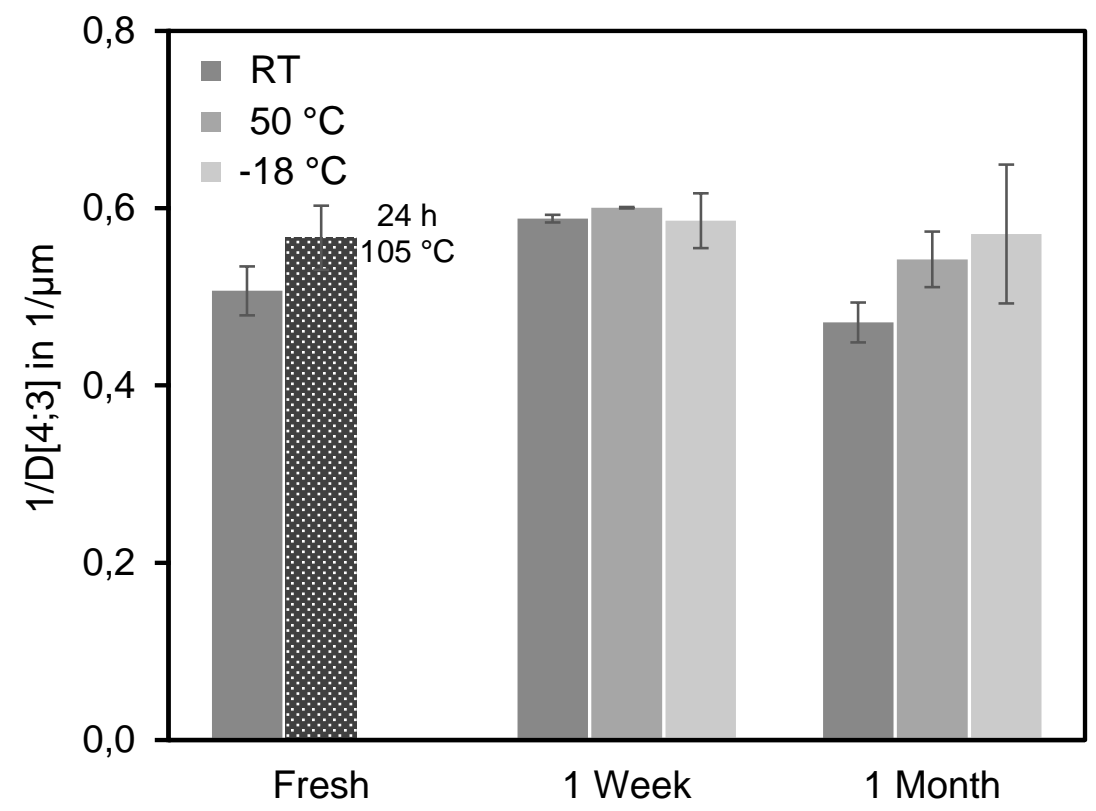

Figure 4.4.21: Effect of different storage conditions on the mean degradation rate

Keeping the freshly produced silica in an oven at $105^{\circ} \mathrm{C}$ surprisingly increased the mean degradation rate instead of reducing it as shown in the previous long-term study when silica was stored at $50{ }^{\circ} \mathrm{C}$. After one week hardly any differences due to the storage conditions can be seen. After 1 month, the mean degradation rate of the silica stored at higher temperature $\left(50^{\circ} \mathrm{C}\right)$ as well as at lower temperature $\left(-18{ }^{\circ} \mathrm{C}\right)$ seems to increase in comparison to the RT sample. Due to the high deviations measured it is most likely that a measurement error occurred. Either the measurement system was polluted or the wear of the sonotrode has progressed too far. Therefore, these results do not seem to be reliable.

\subsubsection{Concerns and Reservations}

The use of the Fraunhofer and Mie theory as well as the use of laser diffraction systems for particle size analysis is highly discussed in the literature [6, 13-14] and the recommendations are often contradictory. Both approaches contain assumptions which are either not applicable when using silica or exact information about the optical material parameters are missing. For example: Silica has a fractal structure not meeting the requirement for spherical particles. Therefore, it is questionable how they align within a turbulent flow. In addition, sharp edges of the samples influence the beam and therefore cause a diffuse scattering pattern which is more difficult to interpret. It is also not clear how the used algorithms are calculating the exact particles sizes and if they are valid. Correlations with other particle size techniques such as optical imaging lead to very poor results as shown by Eshel et al. [13].

Beside the measurement technique the storage effect of silica shows influences on the final results and has therefore be investigated in more detail. Moreover, it is not clear how strong the effect of the wear of the sonotrode is. The energy input therefore has to be monitored. 


\subsubsection{Conclusion}

The present chapter introduces a new analytical method to determine the easiness and speed of cluster breakage of silica during sonification by means of a laser diffraction particle size measurement. The faster different curve characteristics such as the mean degradation rate and $\mathrm{Dv}(50)$ value change over time, the easier should be the breakdown of bigger clusters. To calculate the particles size distribution from the obtained scattering patterns the Fraunhofer theory is used, although this approximation has certain limitations which has to be taken into account. This method is based on the lasergranulometry [1] and was adjusted to shorten the measurement time, prevent the sonotrode from fast wear and gain more information about the particle size reduction. This new method including changed evaluation is named the in-situ cluster fragmentation. It shows a sufficient repeatability and it is possible to differentiate between different types of silica. The final result is mainly dependent on the ultrasonic treatment. Varying the sample mass, flow velocity and temperature of the suspension have a minor impact.

As a conclusion, it can be said that the in-situ cluster fragmentation method does not meet all requirements in accordance with the general basics for method development as described in chapter 4.1. Especially the storage of the silica has to be further investigated and the influence due to the wear of the sonotrode has to be checked and monitored in detail. Despite these limitations, this newly developed method can be seen as a suitable analytical measurement system.

\subsubsection{References}

[1] R. Valero; J. Hernandez, WO 03/016215 A1, Method of preparing silicas, silicas with specific pore-size and/or particle-size distribution and the use thereof, in particular for reinforcing polymers (2003)

[2] Malvern Instruments Limited, Mastersizer 3000 User Manual (2013)

[3] P. Jokiel, "Methodenentwicklung zur Bestimmung der

Desagglomerationsgeschwindigkeit a für gefällte Kieselsäuren", Master: 2015,

Fachhochschule Aachen, Jülich

[4] ISO 13320:2009 - Particle size analysis - Laser diffraction methods

[5] A. Jillavenkatesa et al., Particle Size Characterization, U.S. Government Printing Office, Washington, (2001)

[6] R. H. Müller; R. Schuhmann, Teilchengrössenmessung in der Laborpraxis, Band 38, Wissenschaftliche Verlagsgesellschft mbH Stuttgart, Stuttgart (1996)

[7] I. H. Malitson, Interspecimen Comparison of the Refractive Index of Fused Silica, J. Opt. Soc. Am. 55 (1965) 1205-1208

[8] C. Z. Tan, Determination of refractive index of silica glass for infrared wavelengths by IR spectroscopy, J. Non-Cryst. Solids 223 (1998) 158-163

[9] R. K. Iler, The Chemistry of Silica, John Wiley \& Sons, New York - Chichester - Brisbane - Toronto (1978)

[10] ISO 80000-1:2009 - Quantities and units - Part 1: General

[11] HORIBA, a guidebook to particle size analysis (2017)

[12] Internal Information, Evonik Resource Efficiency $\mathrm{GmbH}$

[13] G. Eshel et al., Critical Evaluation of the Use of Laser Diffraction for Particle-Size Distribution Analysis, Soil Sci. Soc. Am. J. 68 (2004) 736-743

[14] R. N. Kelly; F. M. Etzler, What is wrong with laser diffraction?, Donner Technologies, https://onlinelibrary.wiley.com/doi/pdf/10.1002/ps.4526 (2006) 


\section{Chapter 5 - Correlation between Analytical Parameter and In-rubber Dispersion Quality}

In the present chapter the correlation trials between the five standard analytical parameters (CTAB, BET, DOA, moisture content and $\mathrm{pH}$-value) of 25 different types of silica and the measured macro-dispersion qualities (chapter 3) are presented. Moreover, the different evaluation parameters obtained by three newly developed analytical methods: the void volume structure measurement (chapter 4.2), the sedimentation method (chapter 4.3) and the in-situ cluster fragmentation (chapter 4.4) were correlated.

The only relation found to the standard parameters was, that the PAYNE-effect results of the GT80 series without the use of silane correlate to the surface area (CTAB and BET). The higher the specific surface area, the more pronounced the filler-filler interactions respectively a filler network, what can explain a higher PAYNE-effect.

The void volume structure measurement does not show any relation to the level of dispersion at all, whereas the sedimentation method and in-situ cluster fragmentation provides certain indications. Additional investigations with the Dispergrader and a confocal light microscope (CLM) did not provide improved correlation coefficients in comparison to previous measurements. Moreover, the abrasion resistance of eleven selected rubber compounds was measured by means of the Laboratory Abrasion Tester (LAT100) and correlated to the macro-dispersion qualities but no correlation between these parameters could be found.

As a final attempt a data analyzing model (Partial Least Squares - PLS) was used to check if a combination of different parameters can predict the in-rubber dispersibility more sufficiently. The final outcome provides a good indication but to achieve more reliable results the used amount of data sets are too small.

\subsection{Introduction}

The aim of the present work is to find a direct correlation between one single analytical parameter of silica and its in-rubber dispersibility. Therefore, 25 different types of silica with a variety of analytical parameters and dosage forms were investigated by means of standard analytical methods (CTAB [1], BET [2], DOA [3], $\mathrm{pH}$ [4] and moisture content [5]) as well as three newly developed methods (chapter 4). Moreover, all types of silica were mixed into four different compounds (chapter 3); a typical green tire formulation with $80 \mathrm{phr}$ of silica (GT80), a green tire formulation with $50 \mathrm{phr}$ (GT50), a NR (55 phr) compound and a green tire formulation (80 phr) without use of silane (GT80 w/o). For all these compounds the macro-dispersion quality was evaluated by means of two dispersion measurement systems, the Topography Test and the Dispertester. In order to find this direct relation all analytical data sets and in-rubber dispersion measurements were compared with each other. Hence, the present chapter combines the results obtained in chapters $3,4.2,4.3$ and 4.4. Only when a sufficient high correlation is obtained, conclusions to the in-rubber dispersibility can be drawn. Therefore, the Pearson product-moment correlation coefficient $r$ was determined for each possible combination of results. It describes a linear correlation between two sets of values $x$ and $y$ and is calculated as shown in eq. 5.1 [6]:

$$
\boldsymbol{r}=\frac{\sum(\boldsymbol{x}-\overline{\boldsymbol{x}}) *(\boldsymbol{y}-\overline{\boldsymbol{y}})}{\sqrt{\sum(\boldsymbol{x}-\overline{\boldsymbol{x}})^{2} * \sum(\boldsymbol{y}-\overline{\boldsymbol{y}})^{2}}}
$$

Where $\bar{x}$ and $\bar{y}$ are the mean values of the data sets. The values of the correlation coefficient $r$ can vary between +1 and -1 whereby +1 correspond to the best possible positive and -1 to the highest possible negative correlation. A positive correlation means e.g. that the higher the value $x$, the higher the value $y$. Whereas a negative correlation means that the higher the value 
$x$, the lower the value $y$. In accordance with Evans [7] all values higher than +0.79 respectively smaller than -0.79 indicate a very strong dependency of both parameters to each other.

\subsection{Correlation coefficients}

As a first test the five standard analytical parameters of silica were correlated to the dispersion qualities obtained by the Topography Test and Dispertester as well as to the PAYNE-effect values. Moreover, the results of additional dispersion measurement systems as described in chapter 3 were considered. These methods are the Dispergrader and the confocal light microscope (CLM). Eleven selected compounds of the GT80 series with silane were investigated by means of the laboratory abrasion tester (LAT100) to investigate the effect of the macro-dispersion quality on the abrasion resistance. Furthermore, the GT80 series with and without silane were correlated to each other to check if the use of silane affects the general dispersibility of silica.

Finally, an attempt was made to find the desired linear correlation by means of combination of different analytical parameter. For this purpose, a data analyzing model (Partial Least Squares - PLS) was used to predict the dispersibility of silica.

\section{Standard analytical parameters}

To begin with, the five standard analytical parameters of silica (CTAB, BET, DOA, pH and moisture content) were correlated with the dispersion qualities of all four compound series obtained by Topography (peak area in \% and the number of surface defects larger than $2 \mu \mathrm{m}$ ) and the Dispertester (dispersion in \%) as described in chapter 2.4. Moreover, the PAYNEeffect $\left(\Delta G^{*}\right)$ was taken into account as well. Tab. 5.1 depicts the calculated linear correlation coefficients.

\begin{tabular}{|c|c|c|c|c|c|c|}
\hline series & dispersion & CTAB & BET & DOA & $\mathrm{pH}$ & moisture \\
\hline \multirow[t]{4}{*}{ GT80 } & $\Delta \mathrm{G}^{*}$ & 0.73 & 0.62 & 0.26 & -0.17 & -0.03 \\
\hline & peak area & 0.44 & 0.45 & -0.34 & -0.57 & -0.20 \\
\hline & $>2 \mu \mathrm{m}$ & 0.49 & 0.49 & -0.32 & -0.54 & -0.19 \\
\hline & Dispertester & -0.57 & -0.56 & 0.12 & 0.50 & 0.13 \\
\hline \multirow[t]{4}{*}{ GT50 } & $\Delta \mathrm{G}^{*}$ & 0.65 & 0.63 & 0.48 & -0.13 & -0.09 \\
\hline & peak area & 0.63 & 0.60 & -0.26 & -0.45 & -0.01 \\
\hline & $>2 \mu \mathrm{m}$ & 0.64 & 0.58 & -0.26 & -0.39 & 0.00 \\
\hline & Dispertester & -0.22 & -0.25 & 0.21 & 0.15 & 0.29 \\
\hline \multirow[t]{4}{*}{ NR } & $\Delta G^{*}$ & 0.79 & 0.73 & 0.22 & -0.13 & -0.20 \\
\hline & peak area & 0.41 & 0.34 & -0.12 & -0.26 & -0.08 \\
\hline & $>2 \mu \mathrm{m}$ & 0.42 & 0.31 & -0.11 & -0.12 & -0.13 \\
\hline & Dispertester & -0.76 & -0.65 & -0.04 & 0.24 & 0.10 \\
\hline \multirow[t]{4}{*}{ GT80 w/o } & $\Delta G^{*}$ & 0.92 & 0.88 & 0.27 & -0.15 & -0.01 \\
\hline & peak area & 0.19 & 0.21 & -0.43 & -0.61 & -0.16 \\
\hline & $>2 \mu \mathrm{m}$ & 0.39 & 0.39 & -0.39 & -0.60 & -0.13 \\
\hline & Dispertester & -0.58 & -0.56 & 0.12 & 0.52 & 0.04 \\
\hline
\end{tabular}

It can be seen that no direct linear correlation between one single standard analytical parameter of silica and the dispersion quality can be obtained. Therefore, it is not possible to predict the in-rubber dispersibility by means of one of the standard parameters and hence the need for a new analytical parameter respectively measurement system is also shown in these results, as stated before as motivation for the whole study. The only relation found is between the surface area (CTAB and BET) of silica and the PAYNE-effect measurements of series 4 (GT80 w/o). Due to the absence of silane, the silica is not chemically linked to the polymer. 
As a consequence, the surface of silica remains highly polar in contrast to the non-polar rubber matrix. Therefore, a high tendency for reagglomeration arises. The higher the surface area, the higher the chance that filler-filler interactions via hydrogen bonds occur and a strong fillerfiller network is formed. As a result the PAYNE-effect is more pronounced.

In addition to the correlation trials of the dispersion qualities with the standard analytical parameters, the dispersion measurement systems were compared to each other. Therefore, the Topography results (peak area in \% and number of surface defects larger than $2 \mu \mathrm{m}$ ), the Dispertester results (dispersion in \%) and the PAYNE-effect $\Delta G^{*}$ were correlated to each other for every compound series as shown in Tab. 5.2.

Table 5.2: Correlation coefficients of the Topography, Dispertester and PAYNE-effect measurements compared to each other for all four series

\begin{tabular}{|c|c|c|c|c|}
\hline series & dispersion & $\Delta \mathbf{G}^{*}$ & peak area & $>2 \mu \mathrm{m}$ \\
\hline \multirow[t]{3}{*}{ GT80 } & peak area & 0.33 & & \\
\hline & $>2 \mu \mathrm{m}$ & 0.38 & 0.99 & \\
\hline & Dispertester & -0.39 & -0.83 & -0.84 \\
\hline \multirow[t]{3}{*}{ GT50 } & peak area & 0.26 & & \\
\hline & $>2 \mu \mathrm{m}$ & 0.23 & 0.99 & \\
\hline & Dispertester & -0.11 & -0.55 & -0.57 \\
\hline \multirow[t]{3}{*}{ NR } & peak area & 0.47 & & \\
\hline & $>2 \mu \mathrm{m}$ & 0.60 & 0.90 & \\
\hline & Dispertester & -0.80 & -0.62 & -0.78 \\
\hline \multirow[t]{3}{*}{ GT80 w/o } & peak area & 0.10 & & \\
\hline & $>2 \mu \mathrm{m}$ & 0.31 & 0.96 & \\
\hline & Dispertester & -0.52 & -0.69 & -0.79 \\
\hline
\end{tabular}

For all series it can be seen that the peak area and the amount of particles bigger than $2 \mu \mathrm{m}$ show a high correlation coefficient. This is expected due to the fact that both parameters are determined by means of the Topography Test. Moreover, the GT80 series shows a correlation between the Dispertester measurement and the Topography Test. In this case, the two different measurement principles (mechanical and optical) can be compared to each other. For the NR and GT80 w/o series the coefficient values between these systems are higher as well (- 0.78 and - 0.79). The PAYNE-effect measurements only show a slight correlation to the Dispertester results within the NR series (Fig. 5.1) but overall no relation to the macro-dispersion measurement systems could be found.

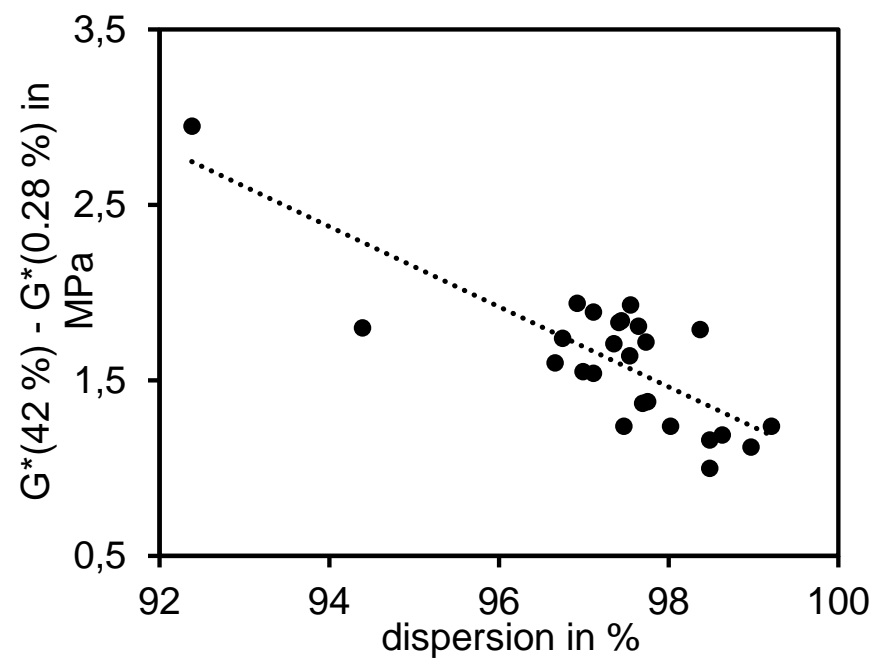

Figure 5.1: Correlation between the PAYNE-effect measurements and dispersion quality obtained by means of the Dispertester for series 3 (NR) 
It can be seen that most values are clouded together in a small area without expressing a real correlation. Only the presence of the two outstanding values lead to a decent calculated correlation coefficient. This fact clearly demonstrates the disadvantage of the calculated values and shows that it is necessary to take the plotted values into account as well.

\section{New analytical test methods}

Previous correlations of the dispersion measurements to the five standard analytical parameters of silica showed, that no direct linear relation exists. This was already stated in chapter 1 and based on these facts the motivation and aim for the present work was formulated. In the next step, the three newly developed analytical methods respectively their evaluation parameters were correlated to the dispersion measurements in order to predict the in-rubber dispersibility of silica. These methods are the void volume structure measurement (chapter 4.2), the sedimentation method (chapter 4.3) and the in-situ cluster fragmentation (chapter 4.4).

\section{Void volume structure measurements}

The void volume structure measurement as developed in chapter 4.2 is an analytical method to describe the initial structure of silica. The evaluation parameter of this system is the void volume value $\left(\mathrm{cm}^{3} / 100 \mathrm{~g}\right)$ at a defined pressure of $5 \mathrm{MPa}$ during compression of the material (VV at $5 \mathrm{MPa}$ ). This parameter was correlated with the macro-dispersion measurements and PAYNE-effect values for all four compound series as depicted in Tab. 5.3.

Table 5.3: Correlation coefficients of the void volume value at $5 \mathrm{MPa}$ mean pressure during compression compared to the dispersion measurements and PAYNE-effect for all four series

\begin{tabular}{ccccc} 
& $\mathbf{\Delta G}^{*}$ & peak area & $\mathbf{>} \mathbf{2} \boldsymbol{\mu m}$ & Dispertester \\
\hline GT80 & 0.38 & -0.22 & -0.19 & 0.02 \\
GT50 & 0.53 & -0.09 & -0.08 & 0.05 \\
NR & 0.42 & 0.03 & 0.05 & -0.18 \\
GT80 w/o & 0.40 & -0.34 & -0.27 & -0.01 \\
\hline
\end{tabular}

As seen from the coefficients values no indication for any correlation between these parameters was found. Fig. 5.2 depicts the void volume values of all 25 types of silica as a function of the measured peak area by means of the Topography Test for the GT80 series.

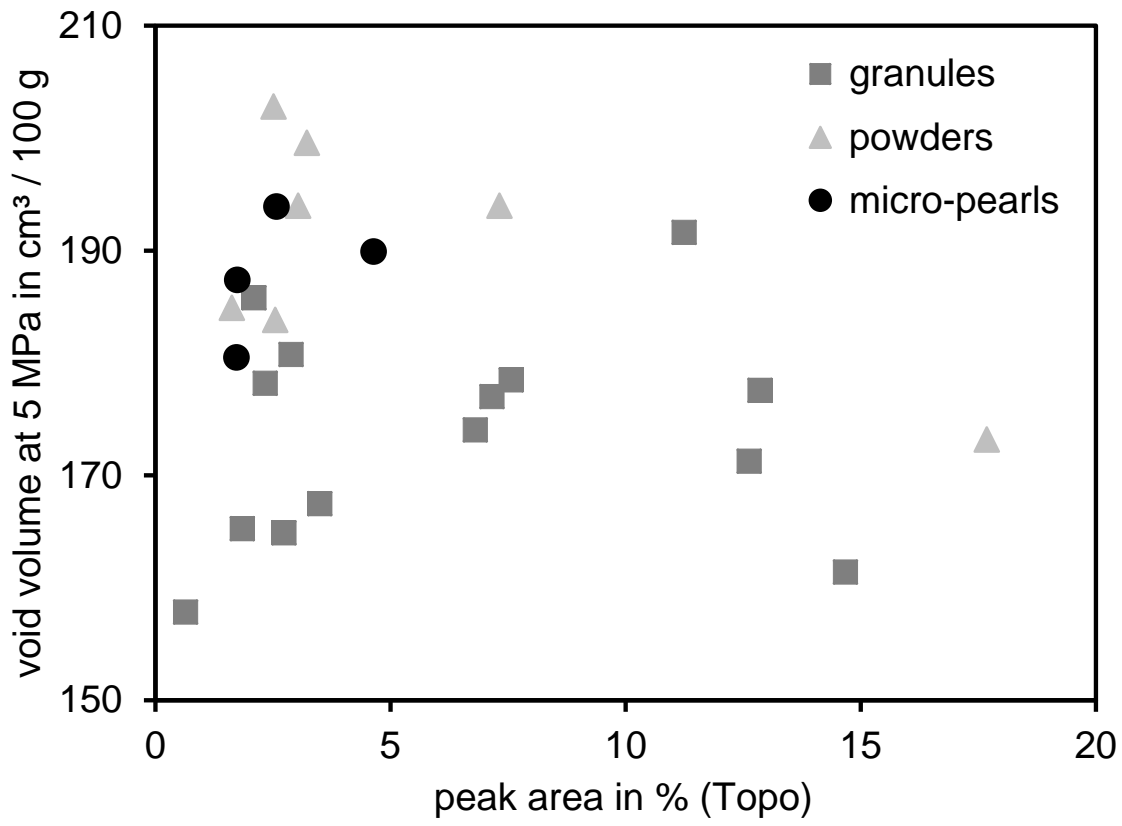

Figure 5.2: Correlation between the void volume measurements and the peak area obtained by means of the Topography Test for series 1 (GT80) 
The data plot emphasizes the obtained low correlation. It is noticeable that powders and micropearls in general exhibit higher void volumes values in comparison to granules. This fact indicates again the impression that the method is dependent on the dosage form and therefore does not meet all requirements for an analytical measurement as described in chapter 4.1. However, even within the different dosage forms no linear correlation is obtained. Therefore, the initial structure of silica seems not to be a main parameter influencing the dispersibility of silica.

As stated in chapter 4.2 the void volume structure measurement can be seen as a suitable alternative to the DOA measurement. Both methods show a high correlation coefficient of 0.92 to each other. However, as previously shown in this chapter, the DOA measurement does not exhibit any relation to the dispersion measurements as well. Therefore, it is not possible to obtain a direct correlation between the void volume structure measurement, respectively the measured initial structure and the dispersion quality of silica. These results are discussed and investigated in more detail in chapter 6.

\section{Sedimentation method}

The sedimentation measurement as developed in chapter 4.3 is an analytical method to measure the particle size and distribution of silica via X-ray absorption after being treated with a defined energy input to reduce the cluster sizes. The obtained results correspond to the amount of particles (in \%) detected, which are bigger than 1 respectively $2 \mu \mathrm{m}$ in diameter. These values were evaluated for all 25 types of silica and correlated to the macro-dispersion measurements and PAYNE-effect values for all four compound series as depicted in Tab. 5.4.

Table 5.4: Correlation coefficients of the sedimentation analysis to the different dispersion measurement methods and PAYNE-effect for all four compound series

\begin{tabular}{|c|c|c|c|c|}
\hline & $\Delta \mathbf{G}^{*}$ & $\begin{array}{c}\text { peak } \\
\text { area }\end{array}$ & $>2 \mu \mathrm{m}$ & Dispertester \\
\hline GT80 $1 \mu \mathrm{m}$ & 0.62 & 0.63 & 0.69 & -0.76 \\
\hline GT80 $2 \mu \mathrm{m}$ & 0.64 & 0.69 & 0.74 & -0.77 \\
\hline GT50 $1 \mu \mathrm{m}$ & 0.29 & 0.74 & 0.81 & -0.62 \\
\hline GT50 $2 \mu \mathrm{m}$ & 0.31 & 0.80 & 0.86 & -0.64 \\
\hline NR $1 \mu \mathrm{m}$ & 0.78 & 0.48 & 0.63 & -0.85 \\
\hline NR $2 \mu \mathrm{m}$ & 0.77 & 0.46 & 0.61 & -0.83 \\
\hline GT80 w/o 1 rm & 0.70 & 0.37 & 0.55 & -0.78 \\
\hline GT80 w/o $2 \mu \mathrm{m}$ & 0.70 & 0.44 & 0.62 & -0.79 \\
\hline
\end{tabular}

It can be seen that most of the obtained coefficients show a relatively high correlation value to the macro-dispersion quality even though $>0.80$ respectively $<-0.80$ is hardly reached. In order to make a more precise and explicit statement, the plotted values were considered as well. Therefore, the best correlation found was taken into account being the amount of particles bigger than $2 \mu \mathrm{m}$ measured by means of the Topography Test (Topo) vs. the percentage of particles bigger than $2 \mu \mathrm{m}$ obtained by the sedimentation method (SG) for the GT50 series (Fig. 5.3). 


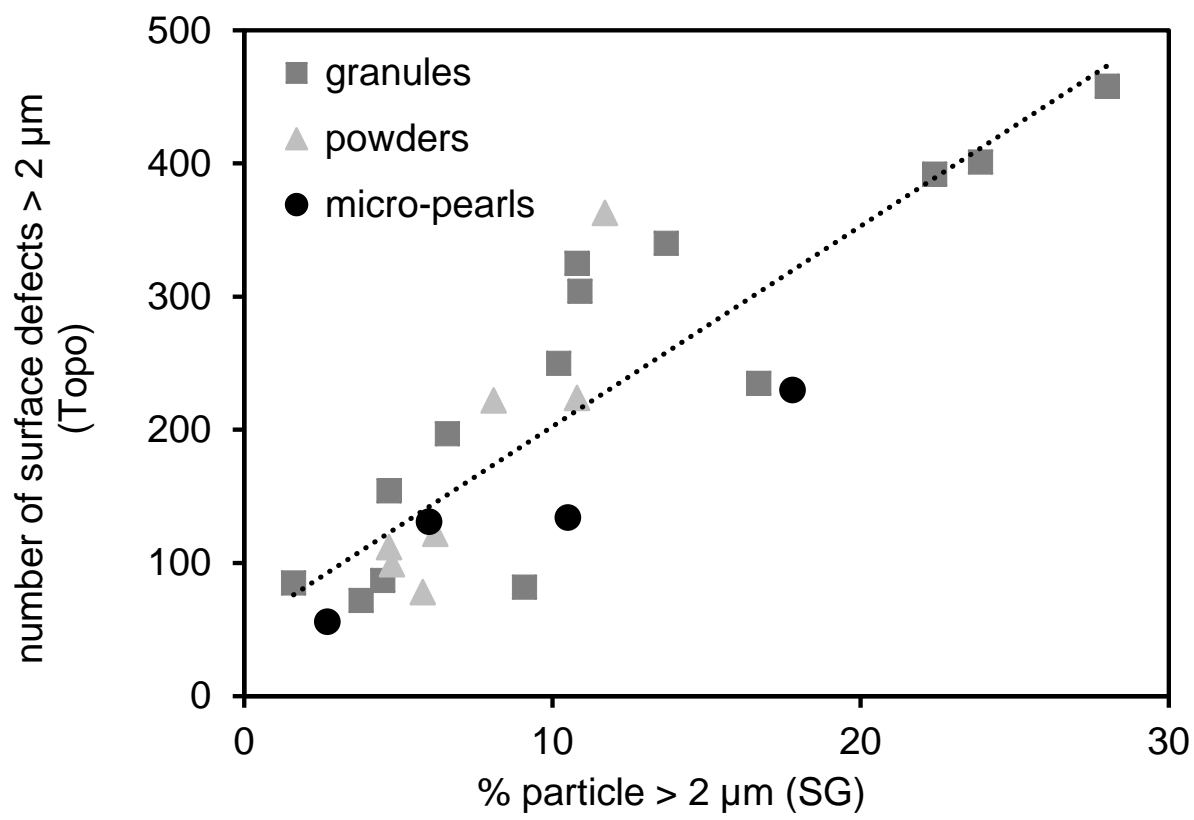

Figure 5.3: Linear correlation between the dispersion quality (Topo) vs. sedimentation values (SG) for the GT50 series

It is shown that even with a correlation coefficient of 0.86 several results do not fit the trend line sufficiently in order to claim the sedimentation method as to be able to predict the dispersion quality of silica in an absolute precious way. One restriction is, that in the present case a counted number of particles in the Topography Test is compared to the volume of particles obtained from SG. However, a defined volume e.g. can either be achieved by a high number of small particles or a lower number of bigger particles. Therefore, a direct correlation of these values cannot be expected.

One conspicuous result was found comparing the Topography Test results (number of surface defects larger than $2 \mu \mathrm{m}$ ) with sedimentation values (\% of particles bigger than $2 \mu \mathrm{m}$ ) for the GT80 series, which was stated to be the reference compound and hence of highest interest. Fig. 5.4 depicts the linear correlation of both measurements.

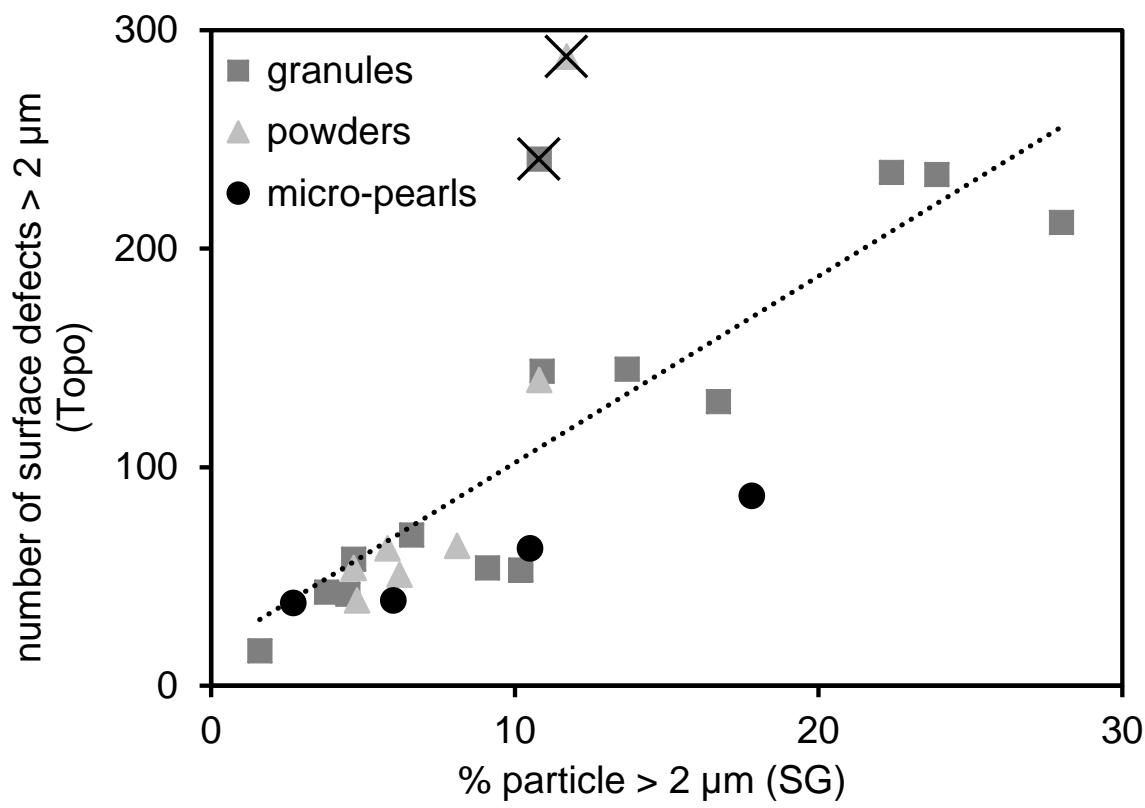

Figure 5.4: Linear correlation between the dispersion quality (Topo) vs. sedimentation values (SG) for the GT80 series 
It can be seen that the overall correlation seems to be very sufficient with the exception of two values having roughly $10 \%$ of the amount of particles bigger than $2 \mu \mathrm{m}$ (SG) but exhibiting a disproportionate high number of surface defects larger than $2 \mu \mathrm{m}$ measured by means of the Topography Test (marked in Fig. 5.4 by an "X"). Neglecting both values in this correlation, the coefficient of 0.74 increases to a value of 0.90 . These two types of silica are related to each other in a sense that these are the powder and granule form from the same precipitation. As a matter of fact, both fillers were produced by a special process which differs from the other 23 samples. However, the requirement to the analytical method being universally valid for all types of precipitated silica cannot be fulfilled by this technique.

\section{In-situ cluster fragmentation}

The in-situ cluster fragmentation (ICF) measurement as developed in chapter 4.4 is an analytical method to monitor the speed of particle size reduction via laser diffraction during an ultrasonic treatment. Various curve characteristics were investigated in chapter 4.4 and compared to find out which is the most suitable parameter to be evaluated to predict the in-rubber dispersibility of silica. Therefore, the progression of the two different mean values $(D[4 ; 3]$ and $\mathrm{D}[3 ; 2])$ as well as the medium particle sizes (Dv(10), Dv(50) and Dv(90)) were considered. Moreover, the desagglomeration rate $\alpha$ [8] was evaluated for all 25 types of silica and finally correlated to the macro-dispersion measurements and PAYNE-effect values for all four series as depicted in Tab. 5.5.

Table 5.5: Correlation coefficients of different curve progression values of the in-situ cluster fragmentation with the measured macro-dispersion qualities and the PAYNE-effect

\begin{tabular}{|c|c|c|c|c|c|c|c|}
\hline series & dispersion & $\alpha$ & $\mathrm{D}[4 ; 3]$ & $\mathrm{D}[3 ; 2]$ & $\operatorname{Dv}(10)$ & $\operatorname{Dv}(50)$ & $\operatorname{Dv}(90)$ \\
\hline \multirow{4}{*}{ GT80 } & $\Delta G^{*}$ & -0.70 & -0.74 & -0.72 & -0.72 & -0.72 & -0.73 \\
\hline & peak area & -0.67 & -0.68 & -0.63 & -0.61 & -0.65 & -0.69 \\
\hline & $>2 \mu \mathrm{m}$ & -0.71 & -0.72 & -0.67 & -0.65 & -0.69 & -0.73 \\
\hline & Dispertester & 0.67 & 0.68 & 0.66 & 0.64 & 0.64 & 0.68 \\
\hline \multirow[t]{4}{*}{ GT50 } & $\Delta \mathrm{G}^{*}$ & -0.53 & -0.56 & -0.58 & -0.59 & -0.59 & -0.54 \\
\hline & peak area & -0.86 & -0.87 & -0.80 & -0.79 & -0.82 & -0.89 \\
\hline & $>2 \mu \mathrm{m}$ & -0.83 & -0.85 & -0.78 & -0.77 & -0.80 & -0.88 \\
\hline & Dispertester & 0.49 & 0.54 & 0.51 & 0.49 & 0.57 & 0.56 \\
\hline \multirow[t]{4}{*}{ NR } & $\Delta \mathrm{G}^{*}$ & -0.61 & -0.66 & -0.62 & -0.61 & -0.62 & -0.68 \\
\hline & peak area & -0.36 & -0.36 & -0.38 & -0.37 & -0.34 & -0.35 \\
\hline & $>2 \mu \mathrm{m}$ & -0.33 & -0.35 & -0.35 & -0.33 & -0.31 & -0.35 \\
\hline & Dispertester & 0.60 & 0.63 & 0.58 & 0.56 & 0.56 & 0.65 \\
\hline \multirow[t]{4}{*}{ GT80 w/o } & $\Delta G^{*}$ & -0.71 & -0.73 & -0.72 & -0.72 & -0.70 & -0.73 \\
\hline & peak area & -0.50 & -0.51 & -0.48 & -0.46 & -0.50 & -0.51 \\
\hline & $>2 \mu \mathrm{m}$ & -0.65 & -0.65 & -0.61 & -0.59 & -0.63 & -0.66 \\
\hline & Dispertester & 0.73 & 0.74 & 0.73 & 0.71 & 0.70 & 0.74 \\
\hline
\end{tabular}

All Dispertester and PAYNE-effect measurements show an insufficient correlation coefficient to the in-situ cluster fragmentation parameters. It is noticeable that the best correlations to the Topography Test were again found within the GT50 series as seen beforehand with the sedimentation method in Tab. 5.4. The GT50 compound dispersion qualities in general demonstrated a wider spreading of results in comparison to the GT80 series as shown in chapter 3.3. This can be explained by the lower degree of filling, hence lower viscosity and less effective dispersive mixing which could also be seen by the differences in power during mixing (chapter 3.2). As a consequence, the energy input applied to silica by means of the sedimentation and in-situ cluster fragmentation methods might be more comparable to the energy input applied during mixing of the GT50 compounds. Fig. 5.5 depicts the highest value of -0.89 between the peak area in \% (Topo) and the Dv(90) progression values. 


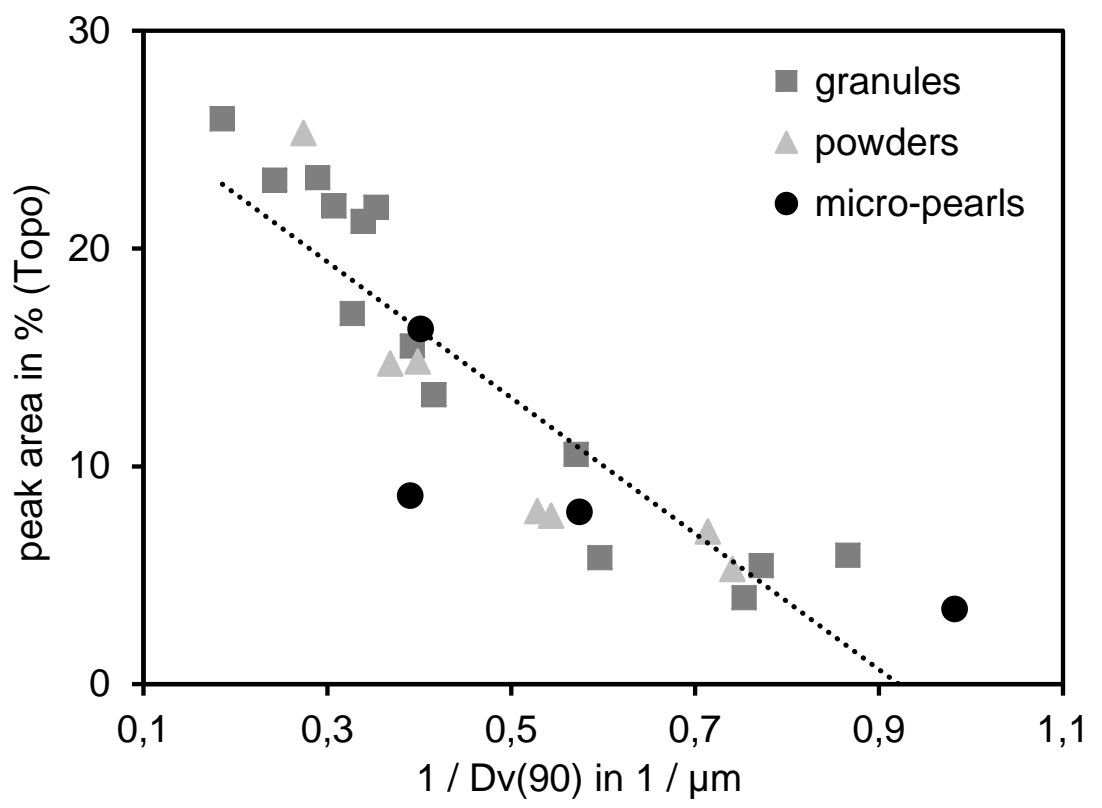

Figure 5.5: Linear correlation between the Dv(90) progression value and the peak area measured by means of the Topography for the GT50 series

With regard to the trend line it is recognizable that the correlation of both data sets is less linear but seems to be more exponential. As a matter of fact, this relation cannot be calculated by means of the Pearson product-moment correlation coefficient $r$ which takes only linear correlations into account [6].

The GT80 series is seen as the reference for all investigations conducted in the present work. The best correlation coefficient found within these compounds was the Dv(90) progression as a function of the amount of particles bigger than $2 \mu \mathrm{m}$ measured by means of the Topography. To evaluate if any indications to the in-rubber dispersibility of silica can be concluded due to the measurement this data set was plotted in the following way (Fig. 5.6):

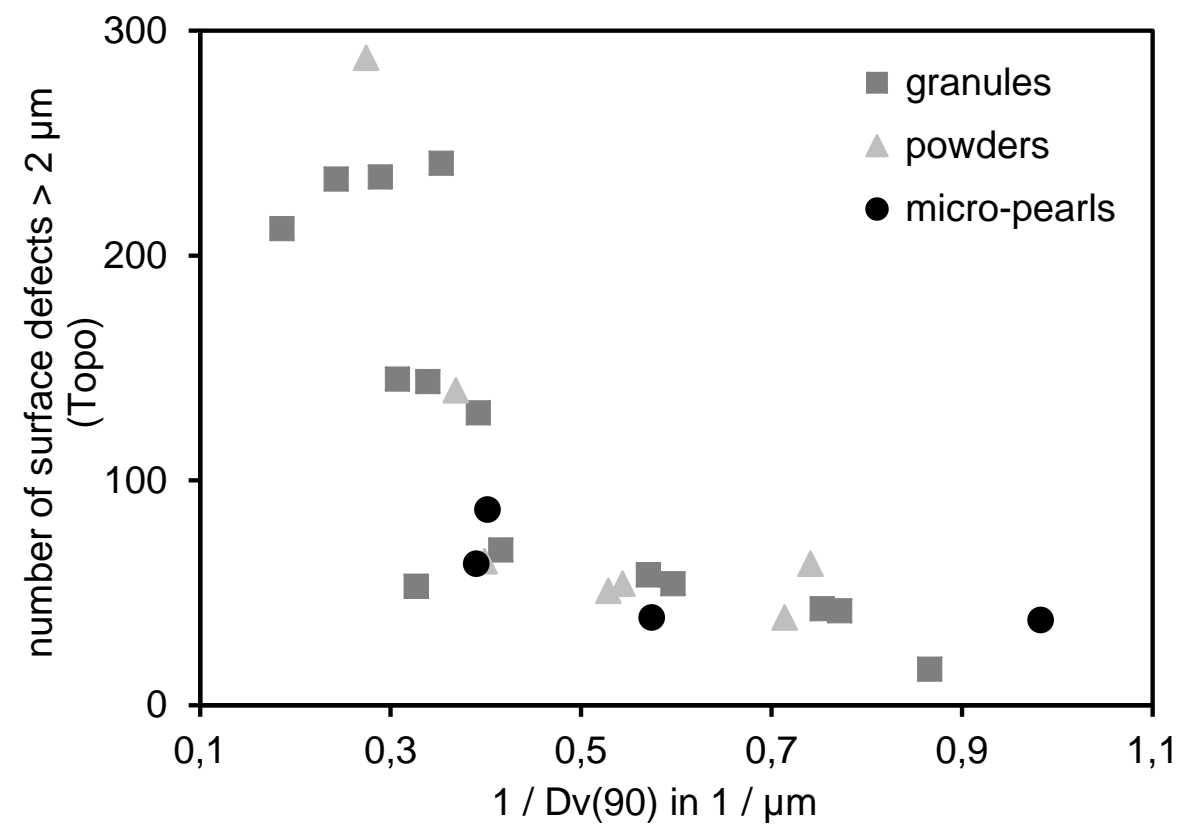

Figure 5.6: Correlation between the Dv(90) progression value and the amount of particles larger than $2 \mu \mathrm{m}$ measured by means of the Topography Test for the GT80 series 
It can be seen that the obtained values possess an even more pronounced exponential relation instead of a linear correlation. Comparing several silica with e.g. a 1 / Dv(90) value of 0.3, very different levels of dispersion are received and therefore, it is not possible to predict the dispersion quality by means of the evaluated analytical parameter. One noticeable result is, that all types of silica possessing a $1 / \mathrm{Dv}(90)$ value bigger than 0.5 show a very good dispersion quality. Therefore, it can be assumed that a high value, at least higher than 0.5 , gives an indication about the dispersibility of silica.

\subsection{Special investigations}

The investigations in this chapter have shown that the newly developed methods, especially the sedimentation method and the in-situ cluster fragmentation, give improved indications to the in-rubber dispersibility in comparison to the standard analytical parameters of silica. As shown in Tab. 5.2 different macro-dispersion measurement systems do not necessarily show a correlation to each other. This fact indicates, that the dispersion measurement systems themselves contain certain errors. Hence, it is more difficult to achieve a sufficient correlation to the analytical measurements. Therefore, two additional dispersion measurement systems, the Dispergrader and the confocal light microscope (CLM), were investigated with selected compounds and correlated to the parameters obtained by the new methods. Finally, the abrasion resistance of several rubber samples were evaluated by means of the laboratory abrasion tester (LAT100) and correlated to the measured dispersion qualities.

\section{Dispergrader}

The Dispergrader is an optical light measurement system to evaluate the macro-dispersion quality of fillers inside a rubber matrix as shown in chapter 2.4.3. To evaluate if it is possible to achieve a better relation to analytical parameters, all compounds of the GT80 series were investigated by this method and correlated to the newly determined analytical parameters (Tab. 5.6).

\section{Table 5.6: Correlation coefficients of the Dispergrader results and the newly developed ana-}

\begin{tabular}{cc}
\multicolumn{2}{c}{ lytical parameters } \\
\hline Value & Coefficient \\
\hline VV@5MPa & -0.06 \\
Sedigraph $>\mathbf{1} \boldsymbol{\mu m}$ & $\mathbf{0 . 7 3}$ \\
Sedigraph $>\mathbf{2} \boldsymbol{\mu m}$ & $\mathbf{0 . 7 2}$ \\
$\boldsymbol{\alpha}$ & 0.56 \\
$\operatorname{Dv}(\mathbf{1 0})$ & 0.53 \\
$\mathbf{D v}(50)$ & 0.53 \\
Dv(90) & 0.59 \\
D[4;3] & 0.58 \\
D[3;2] & 0.55 \\
\hline
\end{tabular}

The highest coefficient found is again related to the sedimentation method. However, the values are too low to claim the system to be sufficient for a clear prediction of the in-rubber dispersibility of silica. This result was expected due to the fact that the direct comparison of the Dispertester and Dispergrader (chapter 3) measurements gave a correlation coefficient of 0.90 . Both systems are based on the similar measurement principle and as well no correlation between the analytical parameters of silica and the Dispertester results was found.

\section{Confocal light microscope (CLM)}

To evaluate the dispersion quality of silica over a wider size range eleven selected silica samples of the GT80 series were investigated by means of the confocal light microscope (chapter 2.4.3 and 3). The level of dispersion was measured at a magnification of 10 and 100 times and the results were correlated to the analytical parameter obtained by the newly developed methods (Tab. 5.7). 
Table 5.7: Correlation coefficients of CLM measurements and the newly developed analytical parameters

\begin{tabular}{ccc}
\hline Value & $C L M \times 10$ & $C L M \times 100$ \\
\hline VV@5MPa & -0.22 & -0.38 \\
Sedigraph $>\mathbf{1} \boldsymbol{\mu m}$ & -0.61 & 0.58 \\
Sedigraph $>\mathbf{2} \mathbf{\mu m}$ & -0.65 & 0.60 \\
$\boldsymbol{\alpha}$ & -0.58 & 0.70 \\
$\operatorname{Dv}(\mathbf{1 0})$ & -0.53 & 0.74 \\
$\operatorname{Dv}(\mathbf{5 0})$ & -0.56 & 0.73 \\
Dv(90) & -0.61 & 0.67 \\
D[4;3] & -0.58 & 0.70 \\
D[3;2] & -0.55 & 0.75 \\
\hline
\end{tabular}

In general, the obtained correlation coefficients are higher for the CLM measurements with a magnification of 100 times in comparison to the lower one. However, even the results of the higher magnification are only on a comparable level to the Dispertester and Topography Test measurements and therefore do not deliver any additional value to the investigations.

\section{Laboratory abrasion tester (LAT100)}

It is stated [9-10] that the abrasion resistance of passenger car tire tread compounds show a direct correlation to the in-rubber macro-dispersion quality of the compound. Therefore, eleven selected compounds (chapter 3.4) with various dispersion qualities were chosen from the GT80 series and tested by means of the Laboratory Abrasion Tester (LAT100). In this way, three different ratings concerning the abrasion resistance could be obtained, which are the total rating, the rating for low severity and the ratio for high severity conditions. These ratings are relative values to one standard compound filled with 80 phr of 159GR204. Tab. 5.8 depicts the correlation coefficients of the three ratings to different macro-dispersion measurements and the PAYNE-effect.

\begin{tabular}{|c|c|c|c|}
\hline Value & $\begin{array}{c}\text { surements an } \\
\text { total rating } \\
\text { in \% }\end{array}$ & $\begin{array}{c}\text { he PAYNE-effe } \\
\text { low severity } \\
\text { in \% }\end{array}$ & $\begin{array}{c}\text { high severity } \\
\text { in } \% \\
\end{array}$ \\
\hline$\Delta \mathbf{G}^{*}$ & 0.85 & 0.79 & 0.82 \\
\hline peak area & 0.04 & -0.12 & 0.23 \\
\hline$>2 \mu \mathrm{m}$ & 0.09 & -0.08 & 0.29 \\
\hline Dispertester & 0.11 & 0.26 & -0.11 \\
\hline Dispergrader & 0.21 & 0.34 & -0.04 \\
\hline CLM x 10 & -0.09 & -0.24 & 0.07 \\
\hline CLM $\times 100$ & -0.43 & -0.38 & -0.50 \\
\hline
\end{tabular}

The only linear correlation obtained from the abrasion ratings are related to the PAYNE-effect measurements (Fig. 5.7) which are said to be an indirect measurement for the micro-dispersion measurements [11] instead of the macro-dispersion. 


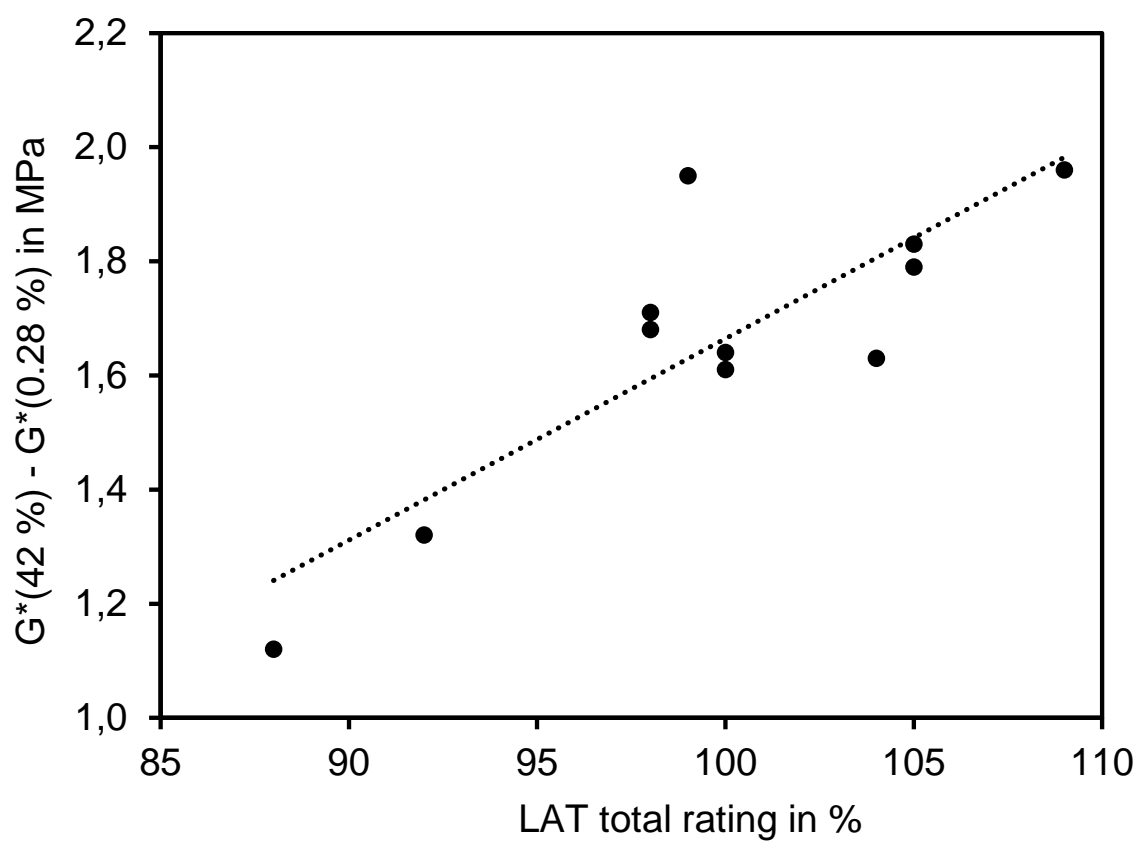

Figure 5.7: Linear correlation between the PAYNE-effect measurements and total abrasion rating obtained by means of the LAT for ten selected samples of series 1 (GT80)

One explanation for this could be that abrasion is a phenomenon which usually occurs at high frequencies [12] related to the chain segment mobility of the polymer which should be influenced by the micro- and not macro-dispersion of the filler. Unexpectedly the LAT rating respectively abrasion resistance seems to be improved with a higher PAYNE-effect and therefore a poorer micro-dispersion quality. However, no statement can be made if the abrasion ratings of the LAT100 measurements represent the real abrasion values occurring on the road.

\section{Correlation between GT80 and GT80 w/o}

A final correlation was made between the GT80 series with and without the use of silane to investigate the influence of the coupling agent to the dispersibility of silica (Tab. 5.9). As shown in chapter 3, the mixing of the GT80 without silane in general results in better dispersion qualities. This can most likely be explained by the fact the low molecular silane partly acts as a plasticizer and lower the compound viscosities. Therefore, less shear forces are generated inside the mixer resulting in a lower effective dispersive mixing.

Table 5.9: Correlation coefficients of the dispersion measurements and PAYNE-effect values for the GT80 and GT80 w/o series

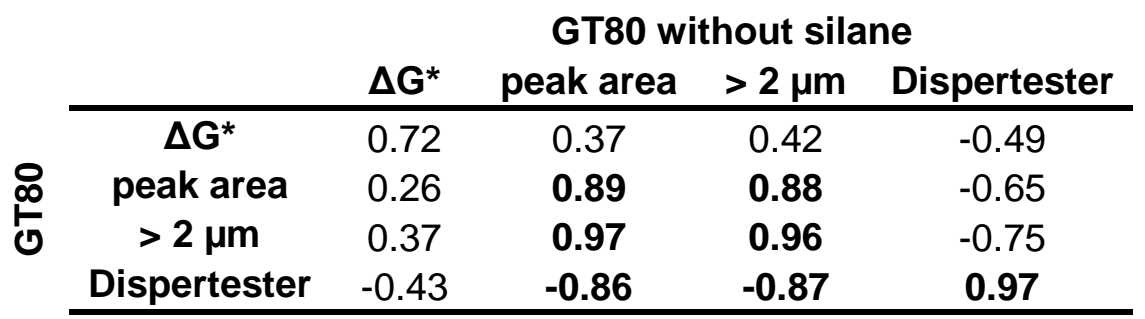

As can be seen, especially the Topography Test measurements (peak area and number of surface defects larger than $2 \mu \mathrm{m}$ ) of both series show a high correlation to each other. Therefore, it can be said that the presence of silane certainly does influence the dispersion quality but not the overall dispersibility of silica. 


\subsection{Data analysis}

The linear correlation of one single analytical parameter to the dispersion quality of silica did not result in a sufficient possibility to predict the in-rubber dispersibility. Therefore, it was investigated if a combination of various parameters leads to a sufficient prediction model. For this purpose, a data based model (Partial Least Squares - PLS) [13] was used to predict the dispersibility of silica. All 25 types of silica were taken into account, including their five standard analytical parameters and newly developed evaluation values. As a target value, which should be predicted, the measured macro dispersion by means of the Topography Test (number of surface defects larger than $2 \mu \mathrm{m}$ ) and by the Dispertester from the GT80 series was chosen.

The Partial Least Squares (PLS) model is based on linear combinations of explanatory factors. These factors are obtained in a way to maximize the covariance between the given data and the target variables [13]. In order to avoid overfitting, the prediction error has been evaluated by the "leave-one-out"-method. This method computes the average prediction error on data points that have not been used for fitting the model to the data. As a first step, a principal component analysis was performed. It turned out that the different dosage forms of silica causes already a high portion of variation in the data. Therefore, the granules were investigated separately from the powders and micro-pearls in order to remove this known variation. The outcome of each following model is the comparison of the actually measured dispersion quality with the obtained results calculated and predicted by the PLS model from the analytical parameters.

To start with, all standard analytical parameters as well as the newly developed analytical parameters were taken into account to find a suitable model for the 13 different silica granules. Fig. 5.8 depicts the correlation of the model to the Topography Test (left-hand side) and Dispertester measurements (right-hand side).

number of surface defects $>2 \mu \mathrm{m}$

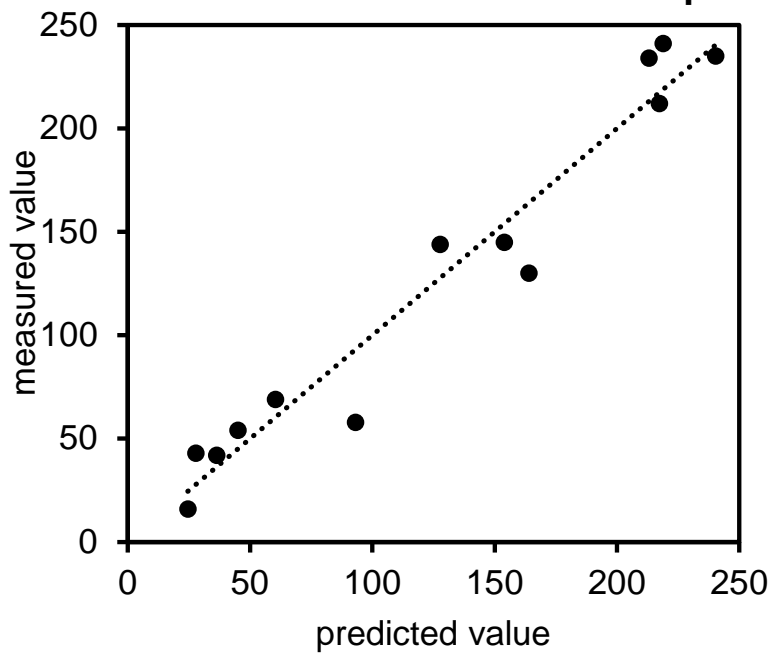

dispersion in \%

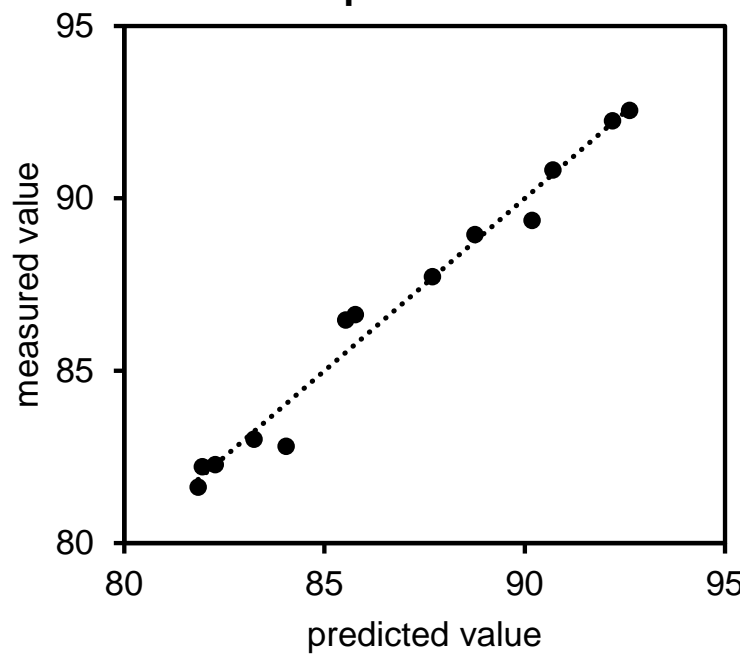

Figure 5.8: Correlation of the model to the Topography Test (left-hand side) and Dispertester measurements (right-hand side) taking all analytical parameters into account

The high linearity respectively low spreading of results indicate that it is possible to find a suitable prediction model considering all analytical parameters. However, taking more than 30 parameters into account is impractical and should therefore be simplified. Reducing the number of explaining factor also contributes to avoid overfitting the model. Hence, as a next step, only the five standard analytical parameters (CTAB, BET, DOA moisture content and pH-value) are considered as depicted in Fig. 5.9. 
number of surface defects $>2 \mu \mathrm{m}$

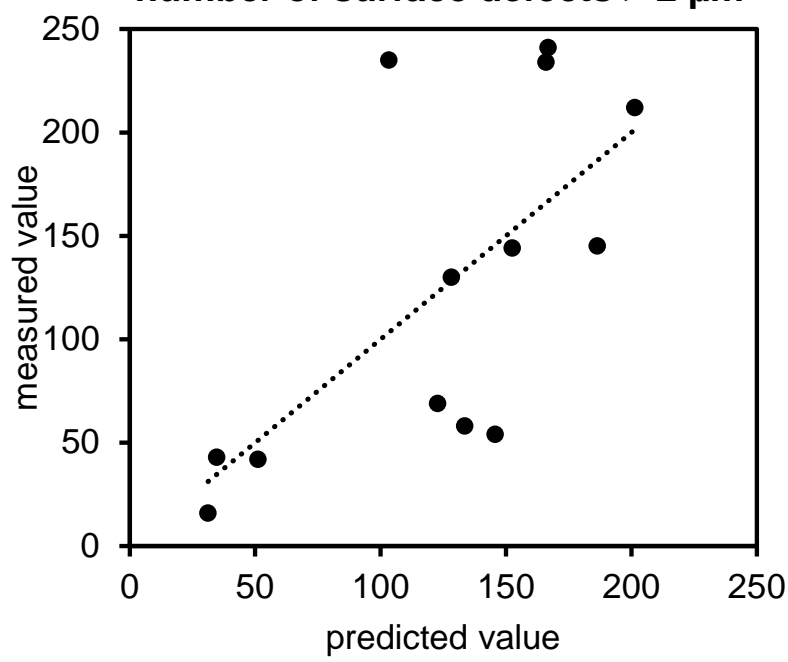

dispersion in \%

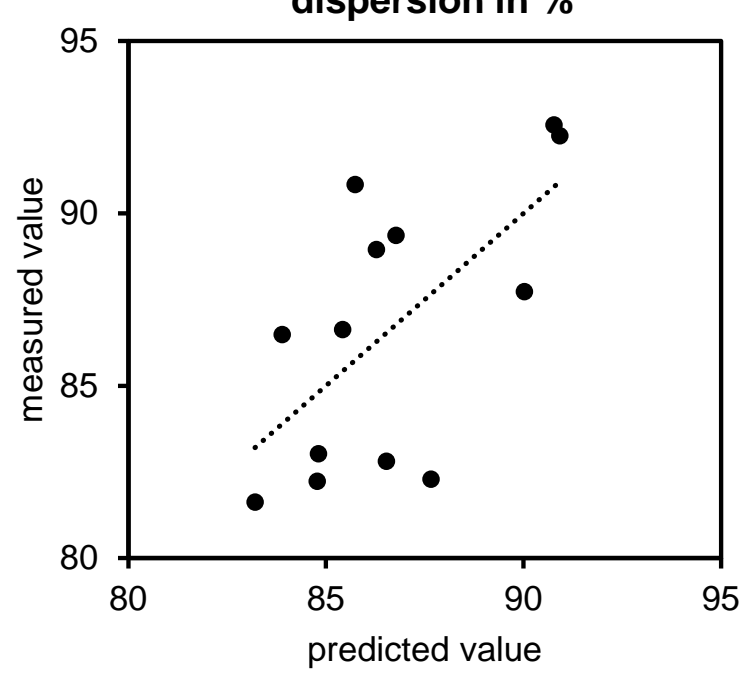

Figure 5.9: Correlation of the model to the Topography Test (left-hand side) and Dispertester (right-hand side) taking only the five standard analytical parameters into account

It can be seen that the best prediction model found with these parameters still contains a high spreading of results which indicates that NO suitable prediction could be obtained based on the given data. This result confirms the statement that it is not possible to predict the in-rubber dispersibility of silica by means of the existing standard analytical parameter. In a last step, only the most influencing parameters obtained by the newly developed sedimentation method (SG) and in-situ cluster fragmentation method (IFC) were taken into account to find a suitable prediction model (Fig. 5.10).
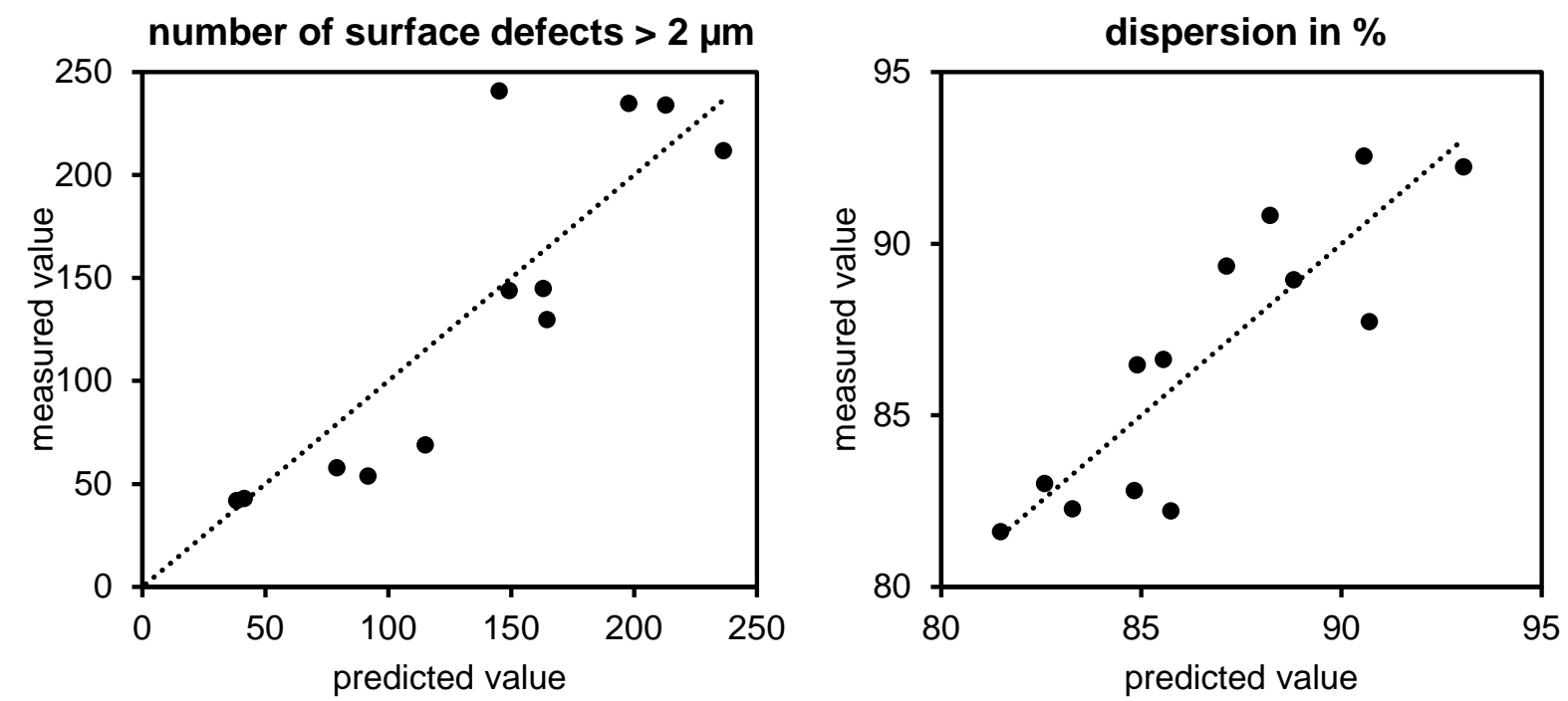

Figure 5.10: Correlation of the model to the Topography Test (left-hand side) and

Dispertester (right-hand side) only taking the sedimentation analysis and in-situ cluster fragmentation into account

It can be seen that the deviations from the predicted to the measured dispersion values are smaller in comparison to the model using the standard analytical parameter. A combination of the analytical silica parameters obtained by the newly developed methods can predict the inrubber dispersibility of silica only to a limited degree. 
This PLS model for the number of surface defects larger than $2 \mu \mathrm{m}$ takes five different parameters into account and can be expressed as the following equation 5.2:

$$
\mathrm{PLS}=516.9-(1.6 \cdot a)-(1.9 \cdot b)-(6021.3 \cdot c)-(72.6 \cdot d)-(38.1 \cdot e) \quad \text { (eq. 5.2) }
$$

with $a$ as the amount of particles $>1 \mu \mathrm{m}$ (SG), $b$ as the amount of particles $>2 \mu \mathrm{m}$ (SG), $c$ as the desagglomeration rate $\alpha, d$ as the $\mathrm{Dv}(90)$ progression value (ICF) and $e$ as the $\mathrm{D}[4 ; 3]$ mean degradation rate (ICF). The achieved equation (eq. 5.2) is only valid within the same formulation and mixing process and cannot directly be transferred to other mixtures. Using this equation, it seems to be possible to predict the in-rubber dispersibility of silica to a certain degree.

As shown in Fig. 5.8 it is possible to find an even more sufficient model with a high linearity respectively low spreading of results taking all analytical parameters into account. Therefore it is necessary to evaluate all parameters concerning the standard as well as new analytical methods and merge them into a comprehensive equation including more than 30 parameters. However, the actual amount of data sets provided is by far too small to generate a statistically validated model. To achieve a reliable result, usually around 100 to 1000 data points are recommended depending on the complexity of the type of model [13].

\subsection{Summary}

In the present work 25 different types of silica with various analytical parameters and dosage forms were investigated. For these fillers the five standard parameters (CTAB, BET, $\mathrm{DOA}$, moisture content and $\mathrm{pH}$-value) were determined as well as different evaluation parameters obtained by the three newly developed analytical methods: the void volume structure measurement (chapter 4.2), the sedimentation method (chapter 4.3) and the in-situ cluster fragmentation method (chapter 4.4). All types of silica were mixed in four different compounds (chapter 3); a typical green tire formulation with $80 \mathrm{phr}$ of silica (GT80), a green tire formulation with $50 \mathrm{phr}$ (GT50), a NR (55 phr) compound and a green tire formulation (80 phr) without the use of silane (GT80 w/o). Various macro-dispersion measurement systems were investigated to evaluated the dispersion quality as well as the PAYNE-effect as to be known as an indirect measurement for the micro-dispersion [11]. Finally, all data were correlated with each other to find the most suitable method to predict the in-rubber dispersibility of silica.

To begin with, the five standard analytical parameters were investigated. The only correlation found was one between the PAYNE-effect results of the GT80 series without the use of silica and the surface area. The higher the specific surface area, the more pronounced the filler-filler interactions respectively a filler network. Therefore, a higher PAYNE-effect can be explained.

In a next step, the three new analytical methods respectively their evaluation parameters were correlated to the in-rubber dispersion qualities. It turned out that the void volume structure measurement does not show any relation to the level of dispersion whereas the sedimentation method and in-situ cluster fragmentation provides certain valuable indications. However, it is not possible to predict the in-rubber dispersibility of silica completely and sufficiently with these methods.

Additional investigations on different dispersion measurement systems were conducted in order to achieve a better correlation. Therefore, the Dispergrader and a confocal light microscope (CLM) were taken into account. However, both systems did not provide improved correlation coefficients in comparison to previous measurements.

Moreover, the abrasion resistance of eleven selected rubber compounds were measured by means of the laboratory abrasion tester (LAT100) and correlated to the macro-dispersion qualities. It was expected that a better macro-dispersion quality results in an improved abrasion resistance but however, no correlation between these parameters could be found. However, a correlation to the PAYNE-effect was obtained which indicates a relation to the micro-dispersion quality. 
As a final attempt a data analyzing model (Partial Least Squares - PLS) was used to check if a combination of different parameters can predict the in-rubber dispersibility more sufficiently. The final outcome provides an improved indication compared to the single correlation. In order to achieve more reliable results the used amount of data sets were too small.

In the upcoming chapter 6 , several reasons will be discussed why a direct linear correlation of one single analytical parameter can hardly be found and why the initial structure of silica obtained by the void volume structure measurement does not give any indication for the dispersibility.

\subsection{References}

[1] ISO 5794:2010 - Rubber compounding ingredients - Silica, precipitated, hydrated - Part 1: Non-rubber tests

[2] ISO 9277:2010 - Determination of the specific surface area of solids by gas adsorption BET method

[3] ISO 19246:2016 - Rubber Compounding Ingredients - Silica - Oil absorption of precipitated silica

[4] ISO 787-9:1981 - General methods of test for pigments and extenders - Part 9: Determination of $\mathrm{pH}$ value of an aqueous suspension

[5] ISO 787-2:1981 - General methods of test for pigments and extenders - Part 2: Determination of matter volatile at 105 degrees $C$

[6] S. M. Stigler, Francis Galton's Account on the Invention of Correlation, Statistical Science 4 (1989) 73-86

[7] J. D. Evans, Straightforward statistics for the behavioral sciences, Pacific Grove, Calif. Brooks/Cole, (1996)

[8] R. Valero; J. Hernandez, WO 03/016215 A1, Method of preparing silicas, silicas with specific pore-size and/or particle-size distribution and the use thereof, in particular for reinforcing polymers, (2003)

[9] S. Uhrlandt; A. Blume, Kieselsäure für den Grünen Reifen - Prozesse, Produkte, Eigenschaften, Kautsch. Gummi Kunstst. 54 (2001) 520-527

[10] A. I. Medalia, Microscopic estimation of carbon black dispersion, Rubber Age (1965) 82

[11] A. I. Medalia, Reinforcement of Elastomers, Encyclopedia of Materials: Science and Technology (2001)

[12] M. Gruendken et al., Liquid Rubber for Safer and Faster Tires - through compound modification and improved filler dispersion, presented at: Tire Technology EXPO, Hanover, Germany (2018)

[13] G. Kaffenberger, Dispersion of Silica - Data Analysis Report, (2018) 


\section{Chapter 6 - Discussion and Conclusion - Investigation of the Morphology of Silica}

The present chapter summarizes different aspects, which limit the general approach to predict the in-rubber dispersibility of silica. Taken all these facts into account it becomes obvious that a linear correlation of one single analytical parameter with the dispersion quality cannot be found.

Furthermore, open questions due to the different behavior of silica and carbon black during the void volume measurement are discussed. Especially the comparable high value at $125 \mathrm{MPa}$ as well as the fact that all types of silica converge at higher pressures and hence cannot be distinguished anymore, were investigated. On the contrary, carbon black possesses different values at $125 \mathrm{MPa}$ depending on the level of branching. To confirm differences between both types of fillers and to preclude changes within the chemistry and morphology of silica, $X$-ray diffraction measurements, FTIR-studies, TEM and SEM images were conducted by measuring the reference samples and those which were compressed during the void volume measurement.

As a final conclusion it is stated that the structure of both types of fillers differ significantly. Carbon Black possesses on the one hand an open fractal structure whereas precipitated rubber silica on the other hand exhibit a dense fractal structure. For the latter a distinction has to be made between the initial structure after production and the in-rubber structure which is builtup during vulcanization by means of bi-functional silanes.

\subsection{Introduction}

Investigations conducted in the present work showed, that the in-rubber dispersion behavior of silica is not dependent on one common single analytical parameter e.g. CTAB [1] or DOA [2] and can therefore not be predicted by a linear correlation. However, by means of the newly developed sedimentation method and in-situ cluster fragmentation an indication for the dispersibility of silica can be obtained. In the following, various aspects are discussed which influence these approaches and show why it is therefore hardly possible to find a suitable correlation, respectively prediction.

The first compromise which was made during the studies was to adjust the amount of silane to the specific surface area (CTAB) of different types of silica to ensure a consistent level of hydrophobation. However, in this way the viscosity slightly varies from compound to compound which affects the dispersion process. Not adjusting the amount of silane to the surface area would on the one hand lead to an insufficient hydrophobation when using not enough silane. On the other hand, a surplus of unreacted silane would decrease the compound viscosity and therefore deteriorate the effective dispersive mixing. Additionally, different surface areas influence the vulcanization system as well, e.g. the higher the surface area or the higher the porosity, the higher the possibility that accelerators are adsorbed. Therefore, different obtained dispersion qualities are not exclusively affected by the properties of silica itself.

Moreover, only $25 \%$ of all silanol groups on the surface of silica can react with silane as shown by Blume et al. [3]. Silica with a higher CTAB surface area therefore contain a higher absolute number of non-reacted silanol groups resulting in a more pronounced filler-filler network, even when the amount of silane is adjusted. This influence is seen in Fig. 6.1 which depicts the measured Mooney viscosity of the GT80 series with silane in accordance with ISO 289 [4] as a function of the amount of silane. 


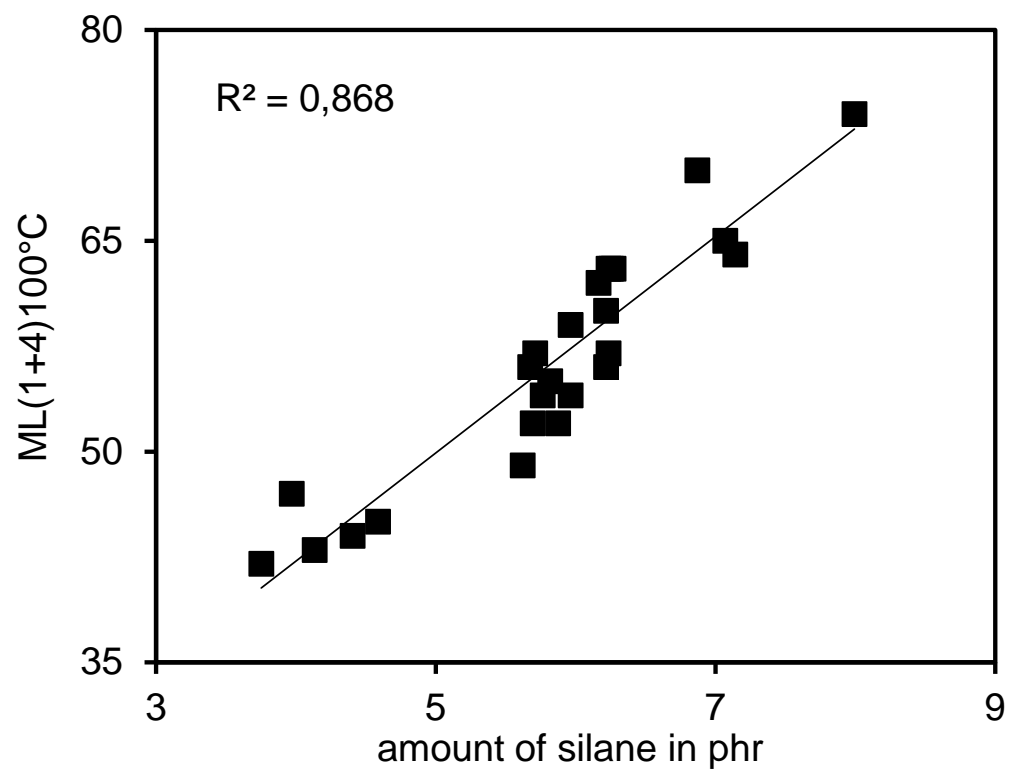

Figure 6.1: Mooney viscosity as a function of amount of silane for the GT80 series with silane

It can be seen that the higher the amount of silane respectively CTAB surface area, the higher is the measured Mooney viscosity. Therefore, silane does not function as a plasticizer in the present case. It is rather likely that the influence of the increasing absolute number of unreacted silanol groups with increasing CTAB surface area, as discussed before, dominates the resulting Mooney viscosity by forming an increasing filler-filler network.

The types of dosage forms (powders, granules and micro-pearls) in general show a different incorporation behavior. Hence, not adjusting the mixing procedure result in a different effective dispersive mixing time which again affects the final dispersion quality results. Therefore, it is difficult to compare different dosages forms with each other.

The different dispersion measurement methods contain certain limitations as well. The Topography Test for instance uses filters to limit the minimum and maximum defect area and roughness values that means the height interval of $2 \mu \mathrm{m}$ to $15 \mu \mathrm{m}$ within defects are counted for the defect area. This is due to the fact that bigger particles do not provide reproducible results anymore [5]. However, such particles might be present and are not taken into consideration. The evaluation by means of the Dispertester and Dispergrader measurements is difficult as well. There is a certain possibility that different particles being close to each other are not detected as separate clusters but as one big particle due to the two-dimensional evaluation. In addition, a good contrast between particles and rubber is needed to measure the dispersion quality by an optical system. Furthermore, all three methods use information about the particle sizes obtained from a freshly cut rubber specimen without knowledge of the full size and shape of different clusters which are partly located underneath the surface.

The rubber compounds consist of roughly 15 different ingredients. Even with a constant mixing procedure and formulation it is hardly possible to achieve precise reproducible in-rubber properties. To show this effect one type of silica (158GR190) was mixed three times into the green tire (GT80) series, at the beginning, in the middle and at the end of the compound series. Tab. 6.1 shows the different dispersion qualities measured by means of the Topography Test (peak area in \% and number of surface defects $>2 \mu \mathrm{m}$ ) and Dispertester (dispersion in \%) for all three batches. 
Table 6.1: Comparison of the dispersion qualities of one compound mixed three times

\begin{tabular}{cccc} 
& batch 1 & batch 2 & batch 3 \\
\hline Topo: peak area in \% & 3.4 & 2.7 & 4.0 \\
Topo: number of surface defects $>2 \mu \mathrm{m}$ & 73 & 58 & 56 \\
\hline Dispertester: dispersion in \% & 89.5 & 89.0 & 88.9 \\
\hline
\end{tabular}

Especially in the case of the Topography results, it becomes obvious that the measured dispersion quality varies within the same mixing procedure, which might be due to deviations within the determination and calculation of peaks and peak areas as well. This variation of results increases the difficulties to find a clear correlation to the analytical parameters of silica and therefore restrict a precise prediction of in-rubber properties.

Even though the sedimentation method and in-situ cluster fragmentation provide clear indications to the dispersibility of silica, they are limited as well. One major reason is the fact that it is not possible to directly transfer the dispersion process into the laboratory. On the one hand, silica is dispersed inside a high viscous rubber matrix (mixer) including different other ingredients like processing oil which influence the dispersion process. In addition, the silane respectively silanization reaction does play a major role for the dispersion process as can be seen when comparing the green tire (GT80) series containing silane with the green tire series without silane (GT w/o) in chapter 3 and 5. On the other hand, the silica is simply solved inside water in both lab approaches to obtain a solution which is treated by means of ultrasound or cavitation. Both processes strongly differ from each other and can therefore not be easily compared.

All these facts lead to the conclusion that the in-rubber dispersibility of silica is influenced by too many factors to be precisely predicted by an analytical parameter. The indications provided by the sedimentation method and in-situ cluster fragmentation are nevertheless a good approach and therefore useful to characterize silica and support the development of new products. However, one open remaining question has to be answered:

Why does the void volume measurement and therefore the structure of silica not show any tendency or relation to the in-rubber dispersibility at all?

Therefore, the morphology of silica and carbon black is finally investigated in the following by various methods. To begin with, the differences between both types of fillers during the void volume structure measurement are highlighted. 


\subsection{Investigations on the morphology of silica}

The void volume structure measurements as investigated in chapter 4.2 show a high correlation to the DOA measurement which indicates that both methods are suitable to determine the initial structure of silica. However, these values hardly draw any conclusion to the inrubber dispersibility of silica (chapter 5 ) whereas the structure of carbon black indeed is somehow related to the dispersion process (chapter 2). Therefore, it is questionable if the structures of both types of fillers are as similar as presumed. A first indication can be given by comparing the void volume measurement curves of different types of carbon blacks and silica as depicted in Fig. 6.2.
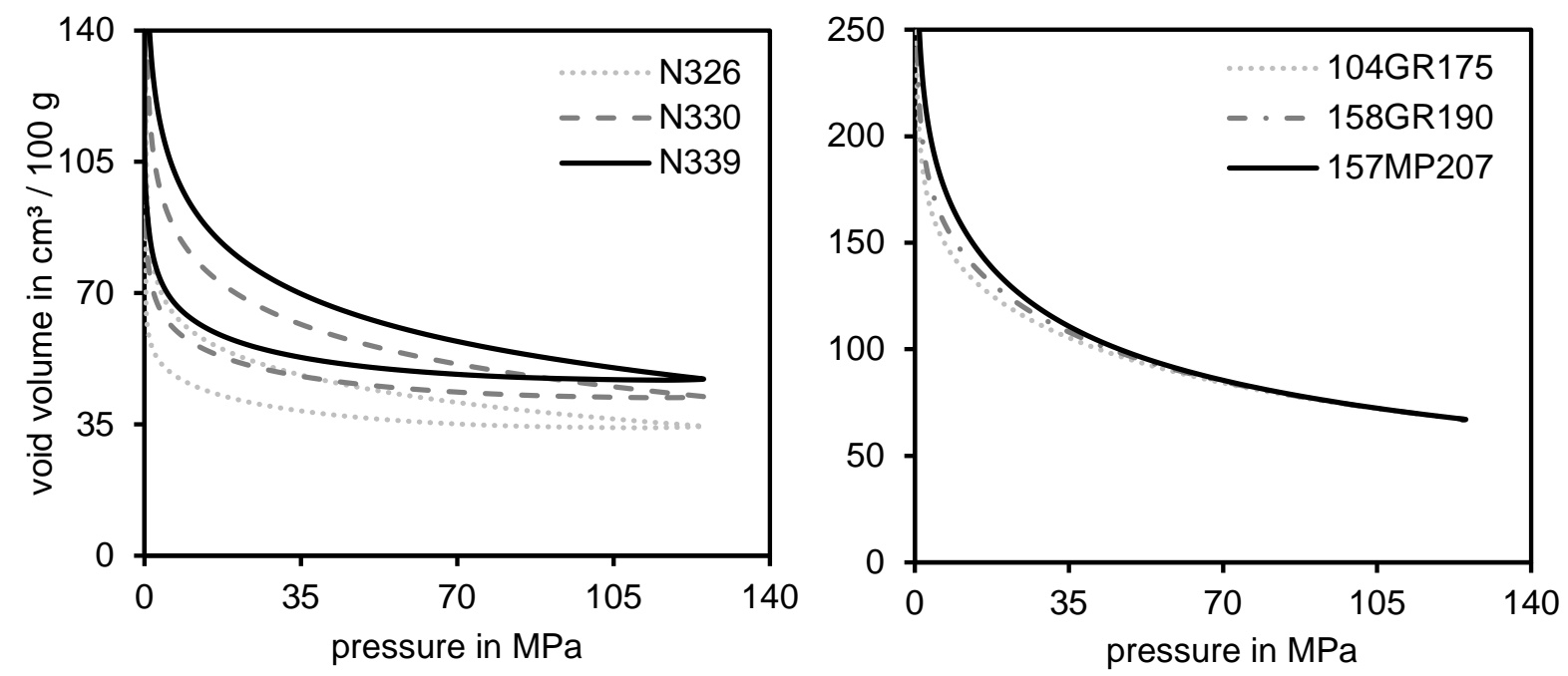

Figure 6.2: Comparison of three different types of carbon black (left-hand side) and three different types of silica (right-hand side) measured by means of the void volume structure tester

The plot on the left-hand side represents the void volume measurements of three different types of carbon black with varied structures measured by OAN [6] and COAN [7]. As described in chapter 4.2, a higher CB structure results in a higher void volume value, especially at high pressures. Different types of silica as depicted on the right-hand side, however, cannot be distinguished at pressure values higher than approximately $50 \mathrm{MPa}$. This phenomenon to reach the same end value can also be found when performing in-rubber tests while measuring the PAYNE-effect (strain sweep) [8] of different types of silica in an identical rubber compound without use of silane. Fig. 6.3 depicts the measured PAYNE-effect curves in accordance with chapter 3.3.3 for three types of silica with different CTAB values as an example. 


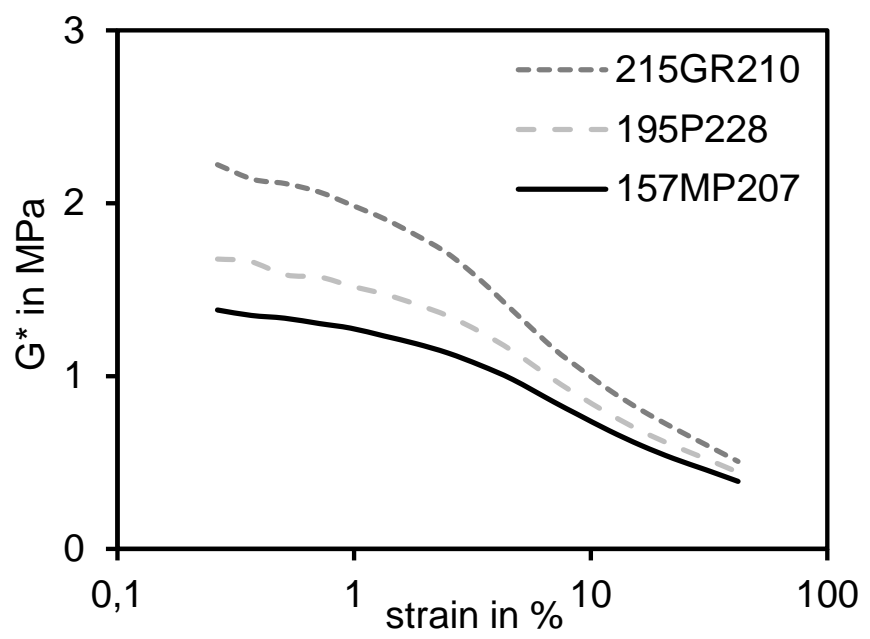

Figure 6.3: PAYNE-effect curves of three different types of silica without the use of silane

It can be seen that a higher surface area leads to higher $\mathrm{G}^{*}$ values at low strains whereas all curves converge to an almost similar end value at higher strains, even with different structure values in case of 195P228 and 157MP207. In contrast to this, CB curves differ at higher strains due to their different structures [9]. The final in-rubber structure of silica has therefore to be build-up during vulcanization with the use of bi-functional silanes. Thereby a difference in the PAYNE-effect curves respectively values at higher strains can be obtained [10-12].

It seems to be that the initial structure of silica fully breaks down during shearing and compression. Hence, the question arises if the morphology of silica changes throughout the measurement whereas a residual structure of carbon black remains. Therefore, different investigations on one type of carbon black (N330) and one type of silica (158GR190) in the reference as well as the state after compression (125 MPa) by means of the void volume test were conducted.

\section{X-ray diffraction measurements}

It is known from the literature that amorphous precipitated silica is able to form crystalline structures when being treated with high temperature respectively high pressure [13]. Additionally, the presence of free water can enhance the formation of siloxane-groups by means of condensation reaction. To get a first indication if the morphology of silica changes within the void volume measurement, X-ray diffraction tests in reflection in the range of $5^{\circ}-100^{\circ}(2 \theta)$ were performed (Philips X'Pert X-ray diffractometer). One type of silica (158GR190) was therefore prepared in three different ways and subsequently tested. Firstly, the reference material was tested as a reference without being compressed by the void volume measurement. Secondly, a compressed sample, treated with $125 \mathrm{MPa}$, was investigated and lastly, one sample was first dried at $105^{\circ} \mathrm{C}$ for $2 \mathrm{~h}$ and then compressed with $125 \mathrm{MPa}$ to check whether the free moisture content will affect the final outcome. All samples had to be slightly ground in an agate mortar before measured by means of the X-ray diffraction. Fig. 6.4 depicts the final outcome of the measurement. 


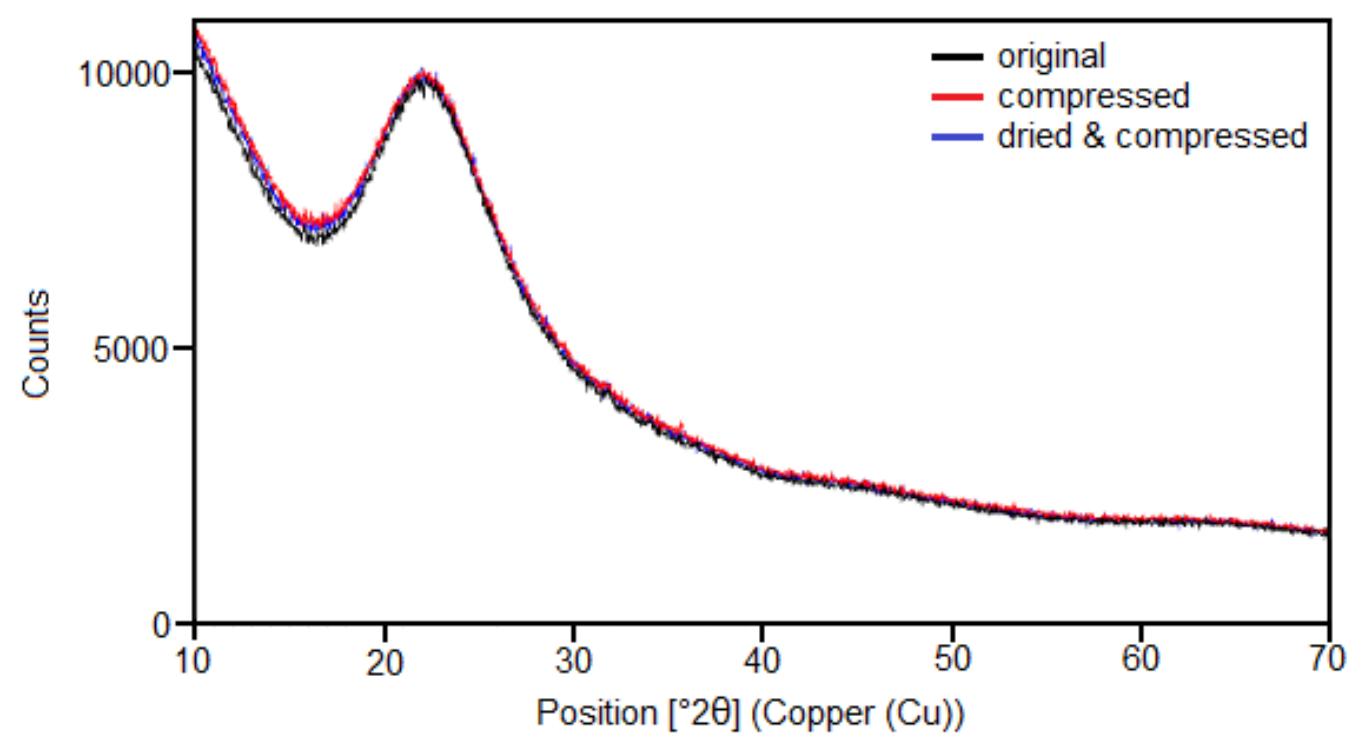

Figure 6.4: X-ray diffraction measurement of one type of silica as the reference, after compression up to $125 \mathrm{MPa}$ and after drying $\left(105^{\circ} \mathrm{C}\right.$ for $\left.2 \mathrm{~h}\right)$ and compression

To achieve a better resolution the samples were measured through a range of angles in the area between $10^{\circ}$ to $70^{\circ} 2 \theta$. As can be seen, all three samples hardly possess any differences, the obtained curves display a typical spectrum of an X-ray amorphous structure which corresponds to amorphous silica. Neither any crystalline response nor a difference between all three samples could be identified. Therefore, no change in the morphology of silica could be determined by means of X-ray diffraction and silica stays amorphous even in the compressed state.

\section{FTIR-study}

To check if a possible condensation reaction occurred during the void volume measurement one type of silica (158GR190) was prepared in four different ways: P1 (reference), P2 (dried at $105{ }^{\circ} \mathrm{C}$ for $2 \mathrm{~h}$ ), P3 (compressed with $125 \mathrm{MPa}$ ) and P4 (dried and compressed with $125 \mathrm{MPa}$ ). These samples were investigated by means of an in-situ Fourier-Transform Infrared Spectroscopy (FTIR) analysis in a static cell (Nicolet 6700 IR spectrometer equipped with a $\mathrm{MCT}$ detector and an extended-KBr beam splitter). As a preparation, all samples were pressed into discs, placed inside the infrared beam and the spectra were recorded. The water content was determined at room temperature under normal atmosphere, whereas the determination of the silanol content and accessibility were performed at $150{ }^{\circ} \mathrm{C}$ and secondary vacuum to remove free water and possible pollutions [14]. First of all, the total amount of silanol groups and the water content were determined as depicted in Fig. 6.5. 


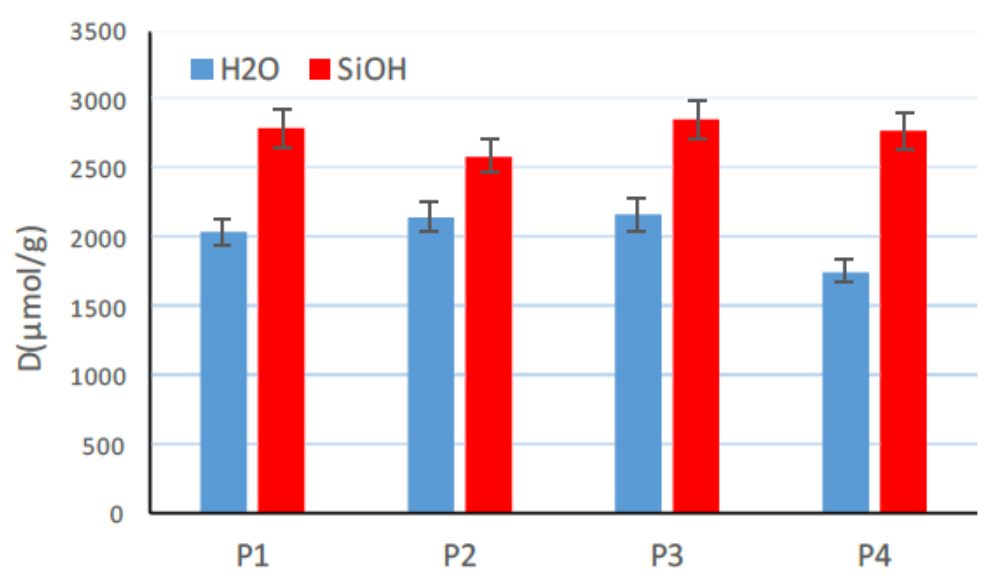

Figure 6.5: Water (blue) and silanol (red) content (in $\mu \mathrm{mol} / \mathrm{g}$ ) of one type of silica in four different forms: $P 1$ (reference), $P 2$ (dried at $105^{\circ} \mathrm{C}$ for $2 \mathrm{~h}$ ), $P 3$ (compressed with $125 \mathrm{MPa}$ ) and P4 (dried and compressed with $125 \mathrm{MPa}$ ) [14]

It can be seen that the total amount of detected silanol groups ( $\mathrm{SiOH})$ does not change due to drying and/or compression by means of the void volume structure tester. This means that the compression of the silica with $125 \mathrm{MPa}$ does not induce any condensation of silanol groups. Surprisingly, the dried sample P2 possesses an identical amount of water $\left(\mathrm{H}_{2} \mathrm{O}\right)$ as the reference sample (P1). Due to the fact that the samples were stored respectively transported between being prepared (compressed) and finally tested there is a possibility that meanwhile moisture was absorbed again. In contrast to this, the compressed and dried sample (P4) possesses a comparable low surface in the compacted state which could diminish the absorption of water in comparison to the undried and compressed sample P3. All in all, these results are an indicator that the surface chemistry of silica did not change and no condensation occurred due to the void volume measurement.

In addition to the determination of the content of water and silanol groups, the accessibility of the silanol groups were investigated. Therefore, different reactants of various sizes were chosen to perform a Hydrogen-Deuterium (H-D) exchange with different alcohols. These H-D exchanged products were investigated by IR spectroscopy due to the fact that this technique is very sensitive to isotropic substitutions. The final $\mathrm{Si}-\mathrm{OH}$ accessibility to the particular alcohols can then be calculated by means of the area of the IR-bands [14]. Tab. 6.2 lists all four reactants including their different molecular mass and size.

Table 6.2: Four reactants used for the $H$-D exchange including their molar mass and molecu-

\begin{tabular}{ccc} 
probe molecules & $\begin{array}{c}\text { lar size [14] } \\
\text { molar mass } \\
\text { in g / mol }\end{array}$ & $\begin{array}{c}\text { molecular size } \\
\text { in } \mathrm{nm}^{2}\end{array}$ \\
\hline 3-ethylpentan-3-ol & 116.2 & 0.41 \\
2-methylpropan-2-ol & 74.1 & 0.32 \\
Methanol & 32.0 & 0.18 \\
$\mathrm{D}_{2} \mathrm{O}$ & 20.0 & 0.11 \\
\hline
\end{tabular}

In Fig. 6.6 the amount of accessible silanol groups relative to the total amount of $\mathrm{SiOH}$ in dependency of the four reactants are plotted for all four silica samples P1 (reference), P2 (dried at $105{ }^{\circ} \mathrm{C}$ for $2 \mathrm{~h}$ ), P3 (compressed with $125 \mathrm{MPa}$ ) and P4 (dried and compressed with $125 \mathrm{MPa})$. 


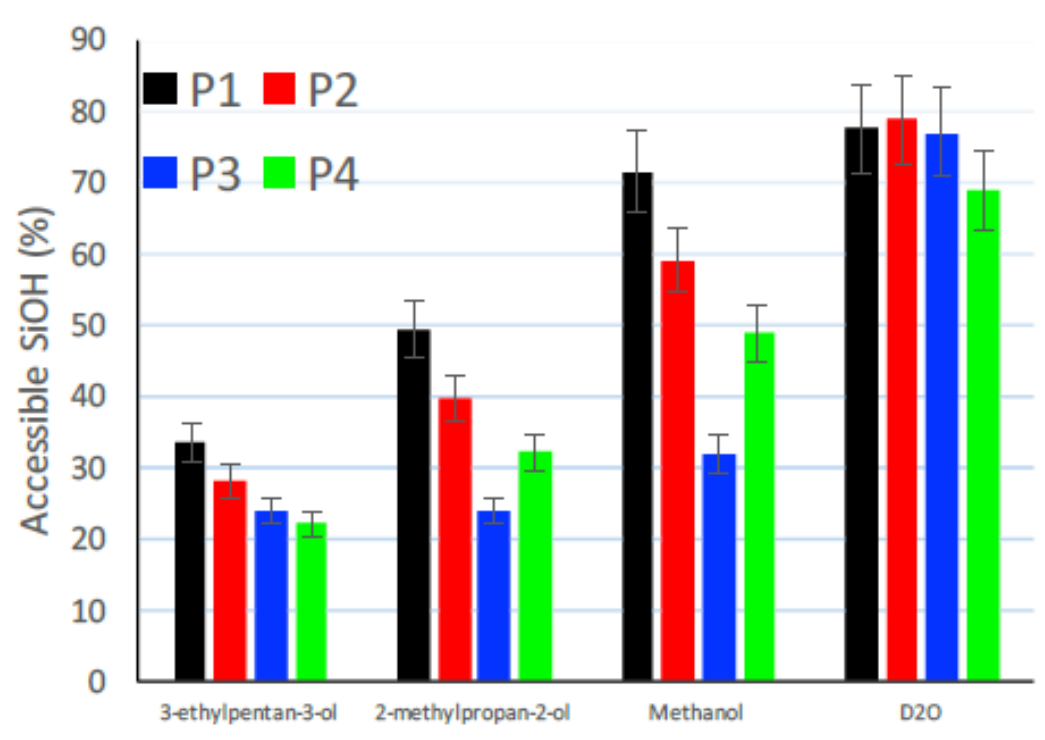

Figure 6.6: Accessible amount of silanol groups (in \%) of 158GR190 in four different forms: P1-black (reference), P2-red (dried at $105^{\circ} \mathrm{C}$ for $2 \mathrm{~h}$ ), P3-blue (compressed with $125 \mathrm{MPa}$ ) and P4-green (dried and compressed with $125 \mathrm{MPa}$ ) [14]

A significant decrease of silanol accessibility towards alcohols is visible between the reference (P1 and P2) and the compressed samples (P3 and P4) whereas the accessibility for the smallest molecule, the heavy water $\mathrm{D}_{2} \mathrm{O}$, is similar. These results provide an indication that a decrease of inter-particular pore sizes occur due to the compression which result in a change in accessibility. As a conclusion, only approximately $30 \%$ of the silanol groups on the surface are accessible to the alcohol, ca. $50 \%$ of the silanol groups are located inside small pores of the silica samples and can only be accessed by molecules smaller than $0.12 \mathrm{~nm}^{2}$. This size corresponds to the size of $\mathrm{D}_{2} \mathrm{O}$ and demonstrates almost the same accessibility for the reference and compressed silica samples. The approximately $20 \%$ remaining silanol groups are supposed to be internal and therefore not accessible at all [14]. These results as well indicate that the chemistry of silica does not change whereas the structure or morphology seems to become denser during compression. However, the approximately $20 \%$ internal silanol groups draw conclusions regarding a high amount of residual cavities inside the samples even after compression. These silanol groups remain even after the compression process which means that the cavity inside the silica structure does not vanish.

\section{Residual structure of different materials}

Assuming that the initial structure of silica fully breaks down during the void volume measurement and that the chemistry of the material does not change during compression, it is expected that the residual structure respectively void volume at $125 \mathrm{MPa}$ is supposed to be relatively small. This should be especially true in comparison to other materials like carbon black with a high expected remaining structure. Therefore, following four materials (Tab. 6.3) were tested by means of the void volume structure measurement and the end values at $125 \mathrm{MPa}$ were compared.

Table 6.3: Four differently structured materials and their density name material

density

in $\mathrm{g} / \mathrm{cm}^{3}$

\begin{tabular}{|c|c|c|}
\hline 158GR190 & $\begin{array}{l}\text { silica - three-dimensional } \\
\text { structured material }\end{array}$ & $2.0[13]$ \\
\hline N330 & $\begin{array}{l}\text { carbon black - three-dimensional } \\
\text { structured material }\end{array}$ & 1.8 [12] \\
\hline Kaolin Advafill-S & clay - two-dimensional layer material & 2.6 [12] \\
\hline Omicron NP3 P0 & $\begin{array}{l}\text { Glass beads - } 2 \text { to } 10 \mu \mathrm{m} \text { in diameter, } \\
\text { non structured }\end{array}$ & $2.46[12]$ \\
\hline
\end{tabular}


Fig. 6.7 depicts the void volume curves of these four materials in accordance with the developed test sequence from chapter 4.2. The density of each sample had to be adjusted for the correct calculation of the void volume values.

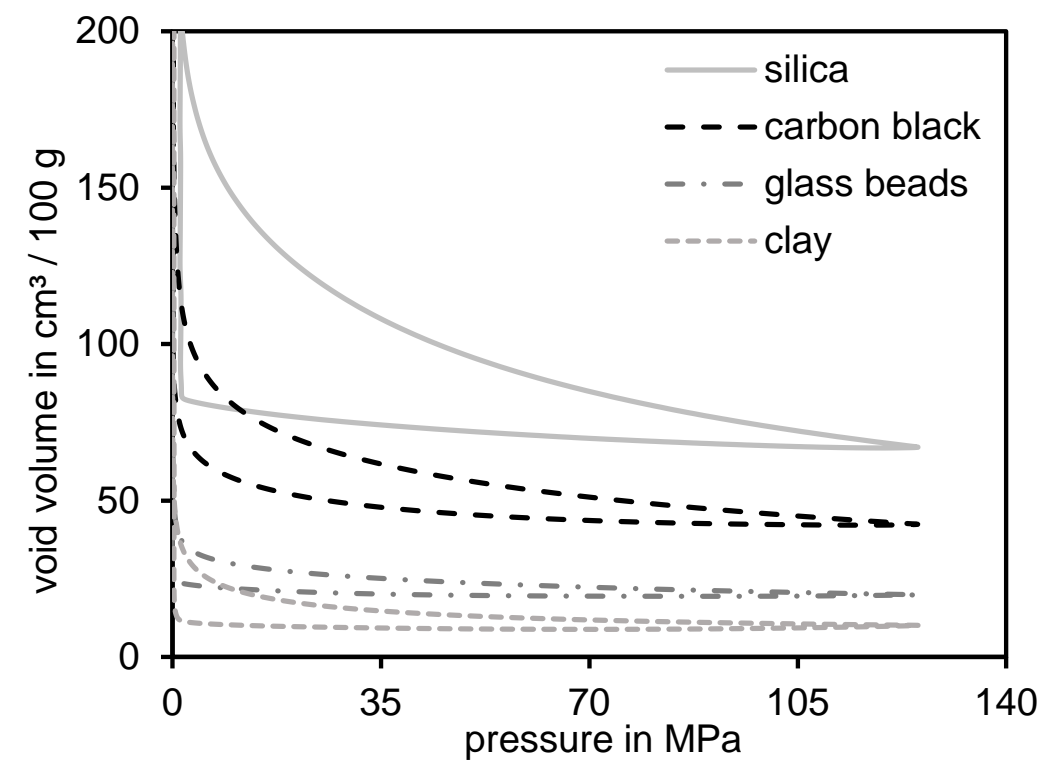

Figure 6.7: Four different structured materials measured by means of the void volume structure tester

It can be seen that the clay possesses the lowest void volume values at $125 \mathrm{MPa}$. This was expected due to the fact that the non-structured two-dimensional layered material is able to be compacted very narrow without many voids left in between. The glass beads show a slightly higher end volume which as well seems to be reasonable. The densest sphere packing still contains a high amount of space in-between the round shape of single beads which only can be filled by either smaller spheres or by destroyed beads. In comparison to these two materials, carbon black shows a higher void volume value at $125 \mathrm{MPa}$. This can be explained by the fact that the initial structure is not fully destroyed during compression and many voids remain inbetween the three-dimensional open fractal structures respectively branches. Silica on the contrary possesses the unexpected by far highest end value of all tested materials. Another peculiarity of these measurement curves is, that carbon black is the only material demonstrating a real hysteresis while being decompressed. The other three materials almost remain in their compressed state. This indicates that carbon black possesses a certain elasticity being able to bend their branches to a certain degree. In the following, different investigations are conducted to find a reason for the high residual void volume of silica.

\section{Isoelectric point of silica}

One possibility why silica is not able to be compressed to a higher extend might be, that it does not appear at its isoelectric point. The isoelectric point corresponds to that $\mathrm{pH}$-value, where molecules do not carry any electrical charge or are electrically neutral. In case of silica, the isoelectric point is around a $\mathrm{pH}$ of 2 whereas most offered types of precipitated silica on the market possess a pH value of 6-7 [13]. That means that silanol groups partly occur in a dissociated form as $\mathrm{SiO}^{-}$respectively $\mathrm{H}^{+}$. Therefore, an electrostatic repulsion might occur which restrict silica to be compressed. To check the influence of the internal charge on the compression, one type of silica (152P287) was produced with three different $\mathrm{pH}$ values and tested by means of the void volume structure tester. Tab. 6.4 depicts the void volume values at 5 and $125 \mathrm{MPa}$. 
Table 6.4: Void volume values at 5 and $125 \mathrm{MPa}$ compression of the silica 152P287 with three different $\mathrm{pH}$ values

\begin{tabular}{ccc}
$\mathrm{pH}$ Value & $\begin{array}{c}\text { VV at } 5 \mathrm{MPa} \\
\text { in } \mathrm{cm}^{3} / 100 \mathrm{~g}\end{array}$ & $\begin{array}{c}\text { V at } 125 \mathrm{MPa} \\
\text { in } \mathrm{cm}^{3} / 100 \mathrm{~g}\end{array}$ \\
\hline 3.8 & 213 & 65 \\
7.5 & 217 & 65 \\
9.2 & 215 & 64 \\
\hline
\end{tabular}

It is shown that the different $\mathrm{pH}$ values do not influence the outcome of the void volume measurement significantly. Especially at $125 \mathrm{MPa}$, all three silica are compressed to the same level. As a conclusion it can be said that the electrical charge of silica cannot explain the high residual void volume in comparison to e.g. carbon black.

\section{Fault in theoretical density of silica}

Another possible reason for the observed difference might be that the calculation of the void volume was wrong. The only parameter which differs between the tested materials is the density. Depending on the referred literature [12-13] the skeletal density values of silica can vary

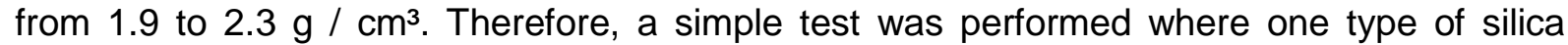
(158GR190) was tested by means of the void volume structure tester (default settings) with varying assumed values for the density. One type of carbon black (N330) was compared to this as well. Tab. 6.5 depicts the calculated void volume at $125 \mathrm{MPa}$.

Table 6.5: Void volume values at $125 \mathrm{MPa}$ compression of one type of silica with three different presumed densities and one type of carbon black (N330)

\begin{tabular}{lcc} 
sample & $\begin{array}{c}\text { Assumed density } \\
\text { in } \mathrm{g} / \mathrm{cm}^{3}\end{array}$ & $\begin{array}{c}\text { VV at } 125 \mathrm{MPa} \\
\text { in } \mathrm{cm}^{3} / 100 \mathrm{~g}\end{array}$ \\
\hline 158GR190 & 1.9 & 65 \\
158GR190 & 2.0 & 67 \\
158GR190 & 2.3 & 74 \\
\hline N330 & 1.8 & 42 \\
\hline
\end{tabular}

It can be seen that the higher the used density value for the calculation, the higher the calculated void volume value at $125 \mathrm{MPa}$. For the calculation of the theoretical volume $V_{T}$ as expressed in eq. 6.1, the density $\rho$ is the denominator whereas the numerator (sample mass $m$ ) remains unchanged [15].

$$
V_{T}=\frac{m}{\rho}
$$

Therefore, a higher density results in a lower theoretical volume. The void volume $V V$ is calculated by the subtraction of $V_{T}$ from the apparent volume $V_{A}$ which remains the same for all three tested samples as shown in eq. 6.2:

$$
V V=V_{A}-V_{T}
$$

Hence, the lower the theoretical volume $V_{T}$ respectively the higher the density $\rho$, the higher the final void volume $V V$. This is confirmed by the experiments. But the significant differences between the silica samples and the carbon black still remains and thus, the high residual void volume at $125 \mathrm{MPa}$ cannot be explained by a fault in the correct quantity of the density. This means that silica still possesses a high residual void volume after being compressed. However, these results seem to be connected to the FTIR-measurements where a high amount of residual cavities could be found within the compressed samples. 


\section{Microscopic investigations}

To get a deeper insight into the difference between the carbon black sample (N330) and the silica sample (158GR190) both materials were investigated by means of transmission electron microscopy (TEM) images and scanning electron microscopy (SEM) technique. For this reason, the reference samples in their initial form and the compressed samples at $125 \mathrm{MPa}$ were evaluated.

\section{Transmission electron microscopy (TEM)}

To measure carbon black and silica by means of a transmission electron microscope (JEOL 2010F with an acceleration speed of $100 \mathrm{kV}$ ) the samples had to be prepared beforehand. They were solved in an isopropanol/water solution and treated by an ultrasonic device with 100 watt for 3 min. In this way, clusters could be separated from each other without breaking interparticular bondings by applying too high energy. Subsequently, the suspension was dried on a fine copper mesh. Fig. 6.8 A to D depicts the TEM images at a magnification of 20.000 for the Carbon Black (CB) N330 and the silica 158GR190 in their initial state and after being compressed by means of the void volume structure tester at $125 \mathrm{MPa}$.
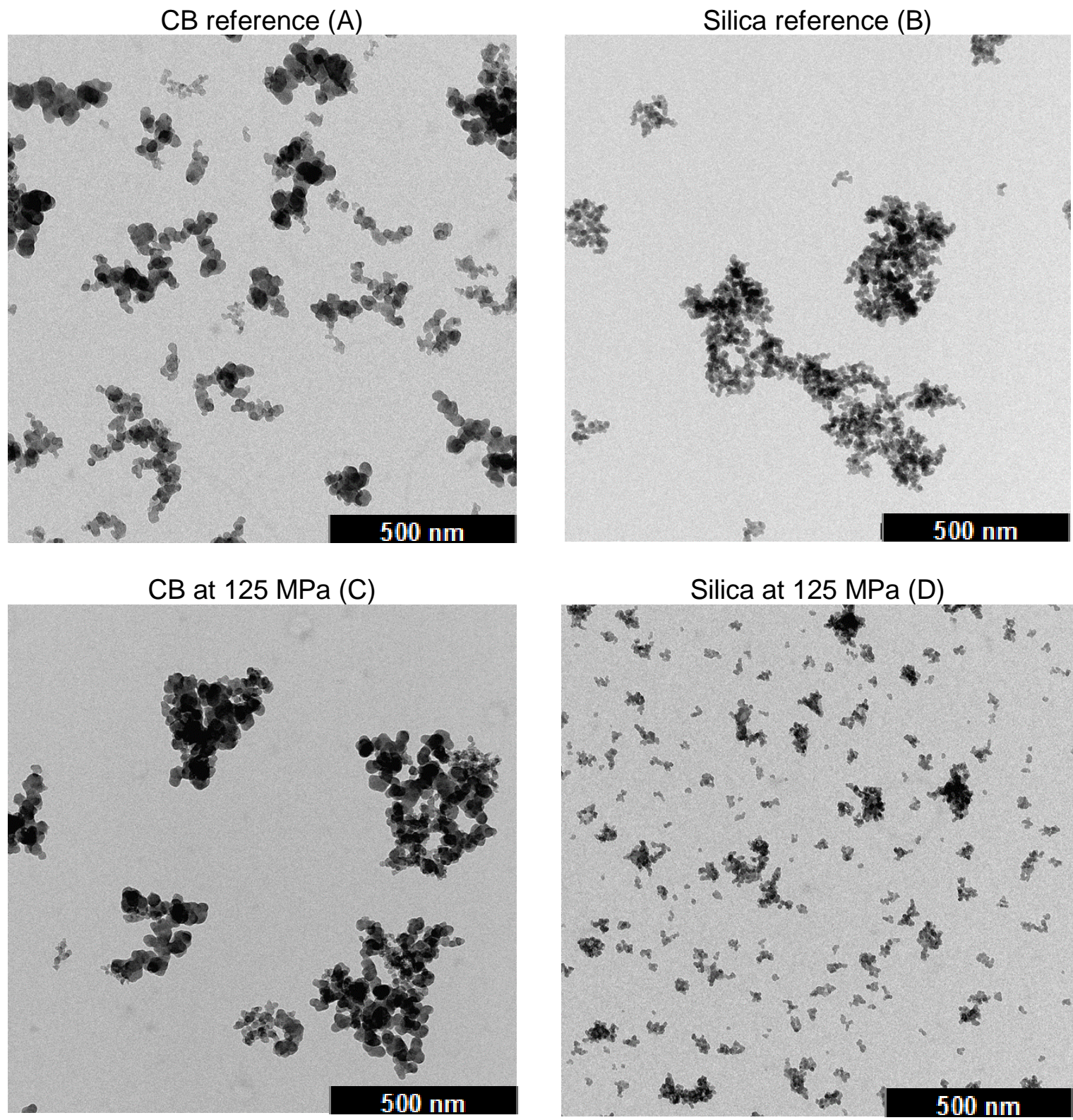

Figure 6.8: TEM images of carbon black $(A)$ and silica $(B)$ in their initial form and carbon black (C) and silica (D) after compression with $125 \mathrm{MPa}$ at a magnification of 20.000 
In their initial state CB seems to be higher branched whereas silica seems to occur as bigger and denser clusters. This can be explained by the high surface polarity of silica forming hydrogen bonds. After the compression, $C B$ clusters tend to entangle whereas silica possesses smaller and even denser formations. A significant difference can be observed in the primary particle sizes where carbon black particles are almost twice as big as silica which can be explained by the smaller surface area of N330 (STSA [16] $=76 \mathrm{~m}^{2} / \mathrm{g}$ ). Fig. 6.9 depicts the same samples at a magnification of 100.000 to get a deeper inside into the structure of the clusters.
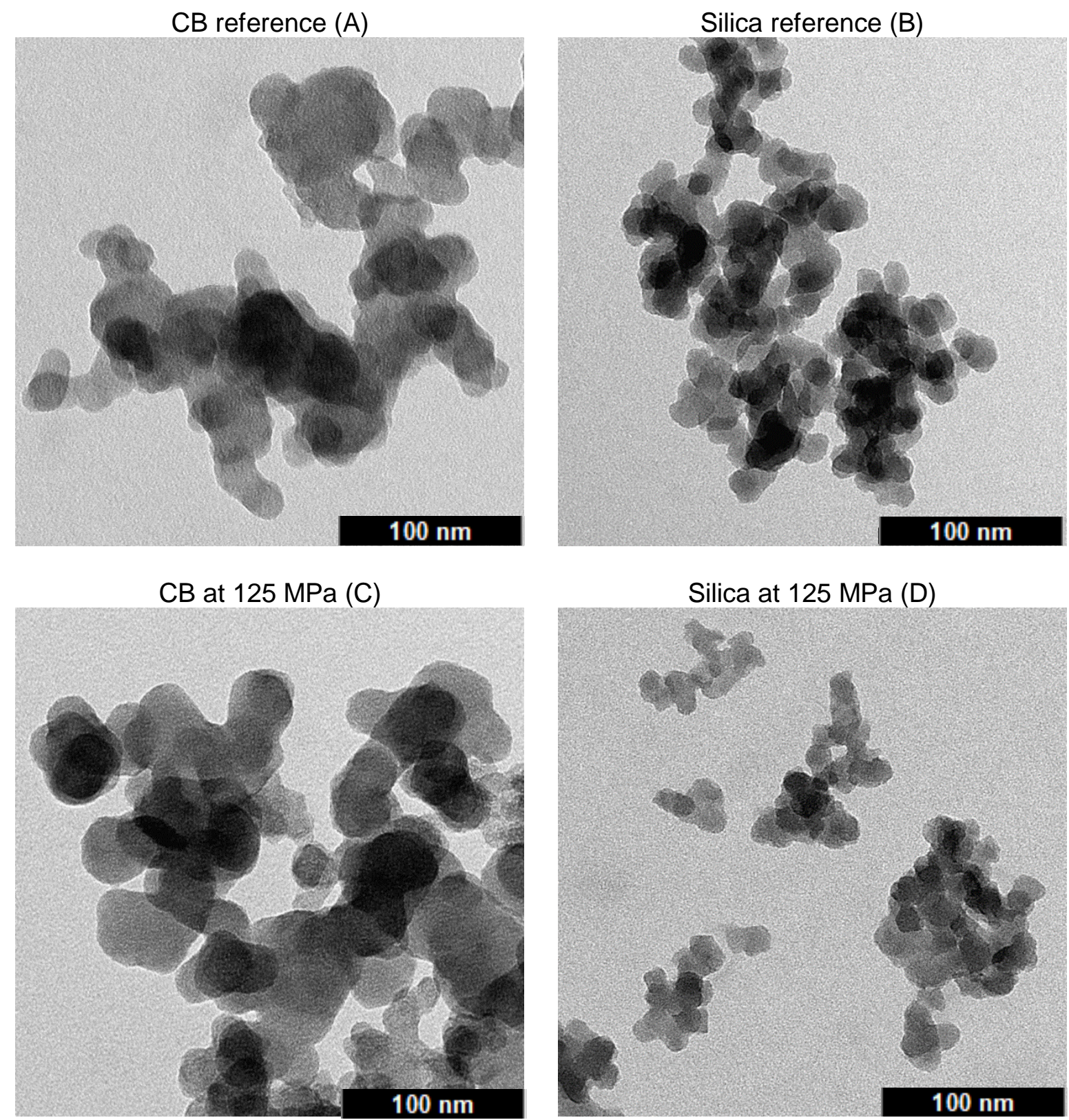

Figure 6.9: TEM images of carbon black $(A)$ and silica $(B)$ in their initial form and of carbon black (C) and silica (D) after compression with $125 \mathrm{MPa}$ at a magnification of 100.000

Comparing the reference samples, carbon black again seems to possess a more open fractal structure whereas silica already show a denser and less branched shape. After compression, the branching of carbon black is still pronounced while the silica clusters have a more grape like shape. 
To achieve an even finer resolution and to make possible crystalline structures visible, the TEM (JEOL 2010F) was changed into an analytical transmission electron microscope (ATEM) with an acceleration speed of $200 \mathrm{kV}$. Fig. $6.10 \mathrm{~A}$ to D depicts the samples of carbon black and silica after compression at $125 \mathrm{MPa}$ at a magnification of 250.000 and 600.000.
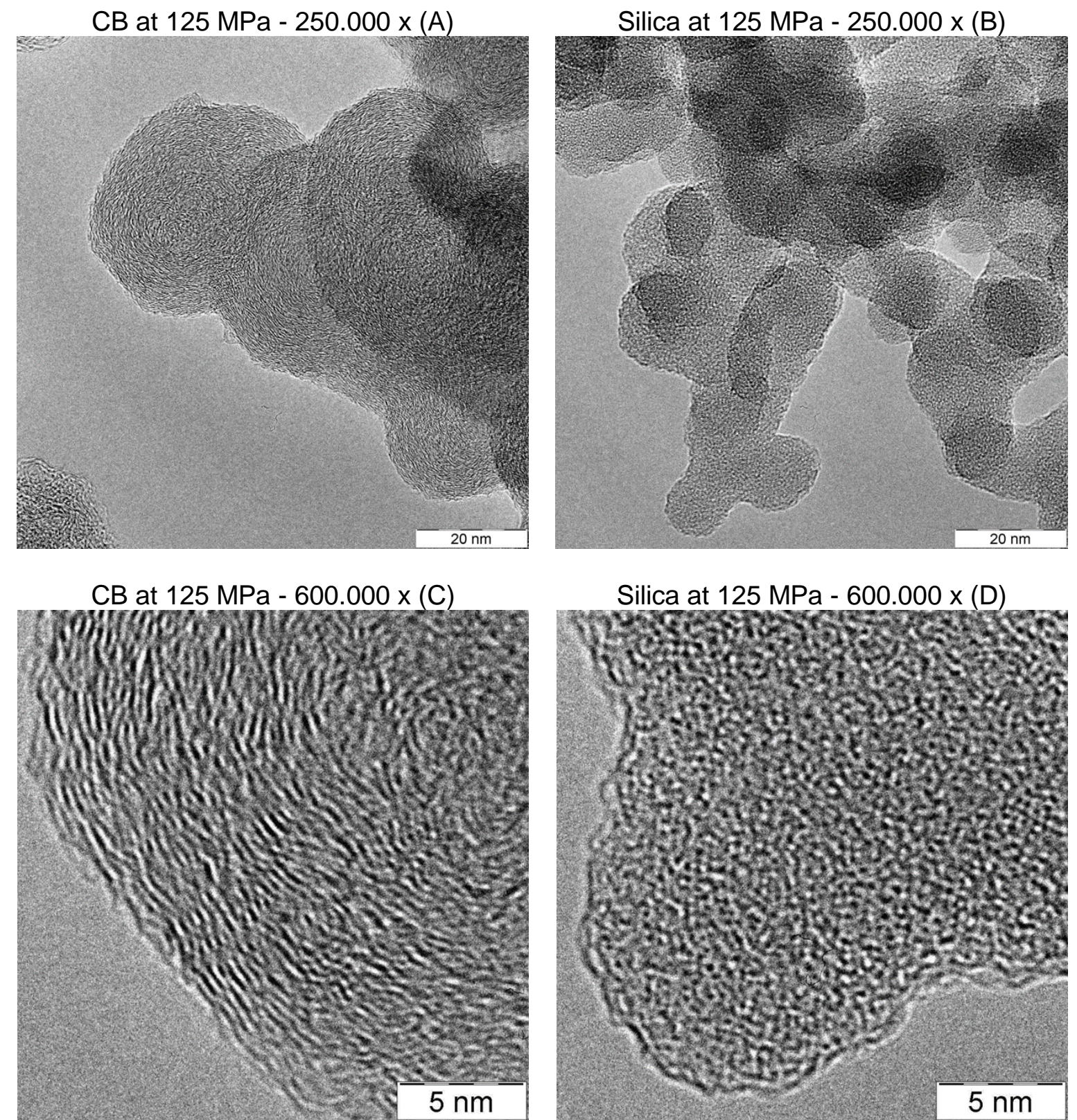

Figure 6.10: ATEM images of carbon black and silica after compression with $125 \mathrm{MPa}$ at a magnification of 250.000 ( $A$ and $B$ ) and 600.000 (C and D)

Especially at a magnification of 600.000 , Carbon Black clearly shows aligned crystalline patterns whereas silica still possesses a fully amorphous structure. Hardly any interfaces between different particles inside the silica clusters can be seen. This is an explicit proof that silica particles do not occur as loosely bonded primary particles which can be separated easily as stated in the literature [17-18] but indeed are bonded chemically to each other to form small clusters as the smallest basic unit. 


\section{Scanning electron microscopy (SEM)}

As an addition to the TEM method, a field emission scanning electron microscope (Jeol 7600F with an acceleration speed of $10 \mathrm{kV}$ ) was taken into account. With this technique, information about the surface of the samples can be obtained. As a preparation, the samples had to be coated by a very thin layer of carbon by sputtering. In contrast to the TEM method, no ultrasonic pre-treatment was performed for the SEM measurements. Therefore, bigger clusters in their initial form could be investigated. Fig. 6.11 depicts the SEM images at magnification of 30.000 and 100.000 for the carbon black N330 and the silica 158GR190 in their initial state and after being compressed by means of the void volume structure tester at $125 \mathrm{MPa}$.

It is shown that differences between the reference and compressed samples are difficult to be judged only by visual inspection of the images. Besides the bigger particle sizes of carbon black it is also hardly possible to differ between both types of fillers. However, a slight tendency of higher amount of voids which are visible inside the silica sample can be presumed.

With the support of the computer software it was possible to evaluate approximately 2000 primary particles and calculate the average diameters of carbon black and silica in accordance to ASTM D3849 [19] in their initial form as well as after compression (125 MPa) from the TEM images as depicted in Tab. 6.6:

Table 6.6: Average primary particles sizes calculated for carbon black and silica in the initial

\begin{tabular}{lcc} 
Sample & $\begin{array}{c}\text { and compressed state }(125 \mathrm{MPa}) \\
\text { Number of evaluated } \\
\text { primary particles }\end{array}$ & $\begin{array}{c}\text { Arithmetical average } \\
\text { in } \mathbf{~ n m ~}\end{array}$ \\
\hline silica reference & 2013 & 12.6 \\
silica at $125 \mathrm{MPa}$ & 2017 & 11.0 \\
\hline CB reference & 2002 & 24.6 \\
CB at $125 \mathrm{MPa}$ & 2002 & 25.8 \\
\hline
\end{tabular}

It seems to be that the average primary particle diameters do not change significantly by means of compression, neither for carbon black nor for silica. The absolute calculated values confirm that carbon black primary particles are twice the size of silica.

In addition to the primary particle sizes also the cluster sizes were investigated and calculated. Approximately 1000 structures were taken into account and the average area, the Equivalent Circular Diameter (ECD), the void area and a branching factor [19] were evaluated (Tab. 6.7).

\begin{tabular}{|c|c|c|c|c|c|}
\hline Sample & $\begin{array}{l}\text { Number of } \\
\text { structures }\end{array}$ & $\begin{array}{c}\text { area } \\
\text { in } \mathbf{n m}^{2}\end{array}$ & $\begin{array}{l}\text { ECD } \\
\text { in } \mathrm{nm}\end{array}$ & $\begin{array}{c}\text { void area } \\
\text { in } \%\end{array}$ & $\begin{array}{l}\text { branch- } \\
\text { ing factor }\end{array}$ \\
\hline silica reference & 1095 & 4282 & 55 & 40 & 8.5 \\
\hline silica at $125 \mathrm{MPa}$ & 1062 & 2030 & 42 & 45 & 5.6 \\
\hline CB reference & 1034 & 17625 & 118 & 37 & 12.0 \\
\hline $\mathrm{CB}$ at $125 \mathrm{MPa}$ & 1044 & 20819 & 133 & 38 & 12.8 \\
\hline
\end{tabular}

As a first impression, it seems to be that the cluster sizes (area and ECD) of carbon black structures are higher than those for silica. The branching of carbon black is more pronounced whereas silica possesses a higher amount of void areas. These results can form a basis for further investigations. 

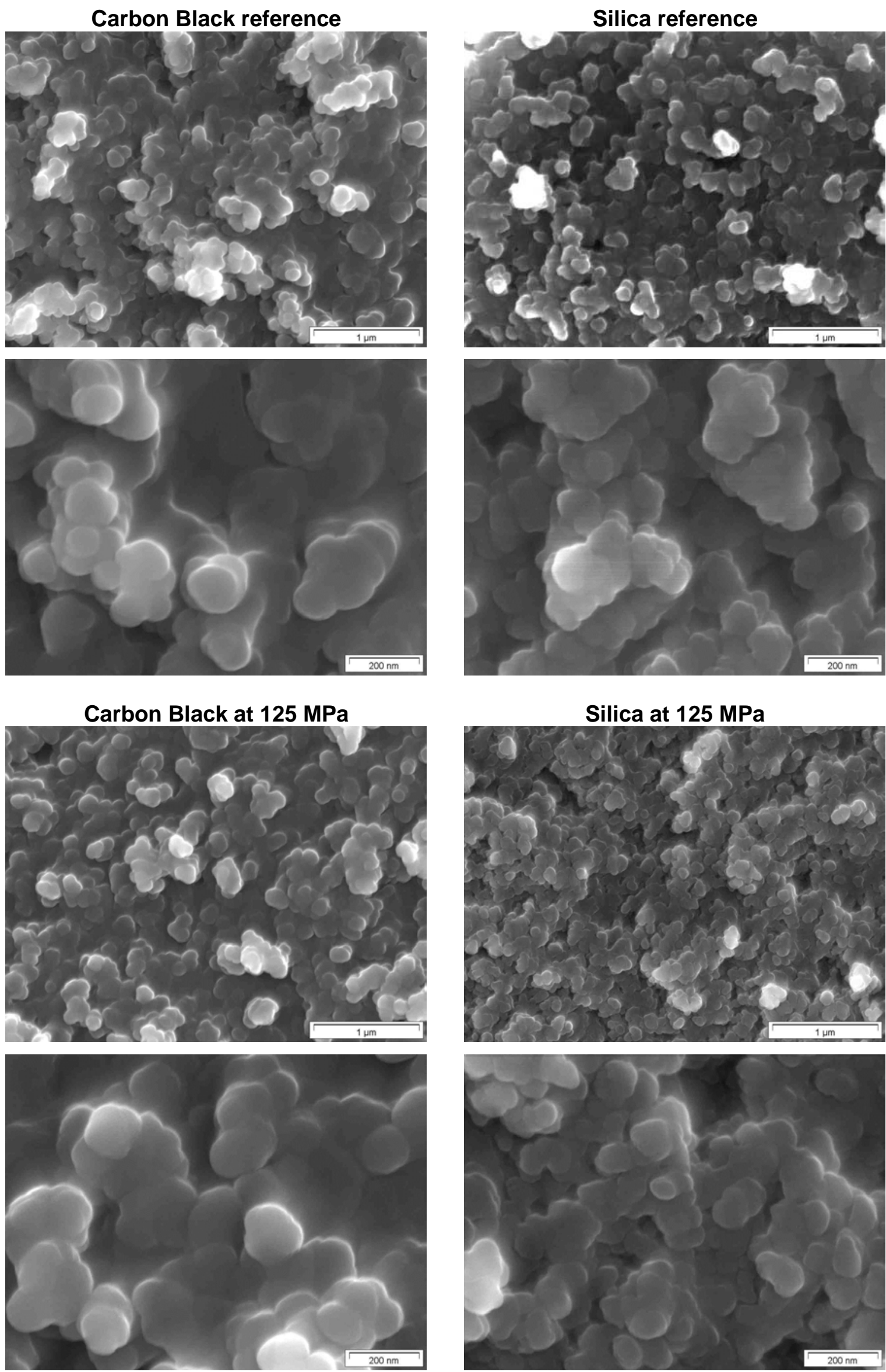

Figure 6.11: SEM images of carbon black (left-hand side) and silica (right-hand side) before and after compression with $125 \mathrm{MPa}$ at a magnification of 30.000 and 100.000 


\subsection{Discussion and Conclusion}

\subsubsection{The Structure of Silica}

Due to the outcome of these investigations it can be said that the structures of carbon black and silica seem to be different. The term "open fractal structure" used for carbon black appears not to be valid for silica. Albers et al. [20] already introduced an alternative terminology for silica, the "dense fractal structure" as depicted in Fig. 6.12.

Carbon Black Cluster

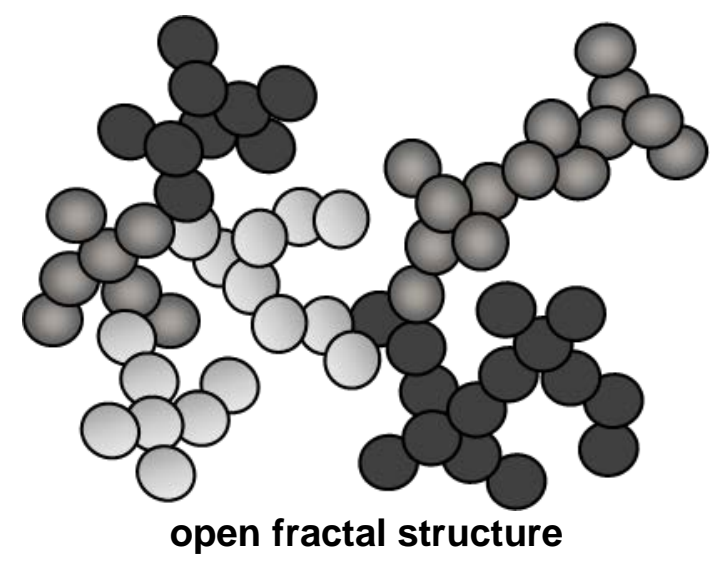

Silica Cluster

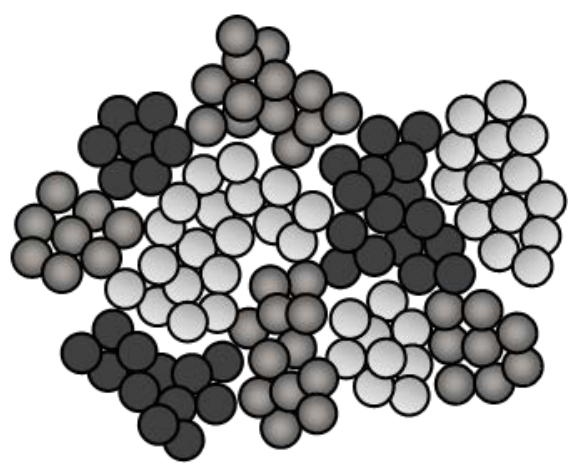

dense fractal structure

Figure 6.12: Schematic view of the differences between the open fractal structure of carbon black and the dense fractal structure of silica

On the one hand, the approach of a dense fractal structure can give an explanation why all types of silica possess an identical void volume value at higher pressures. And on the other hand, it becomes obvious that the results of the void volume measurement does not show any correlation to the in-rubber dispersibility when taken the dispersion process into consideration. Polymer chains are initially able to penetrate into the accessible voids of the silica's structure (Fig. 6.13). Subsequently, shear forces are applied to overcome filler-filler interactions and break down bigger clusters. This seems to be the main criteria for the dispersibility of silica.

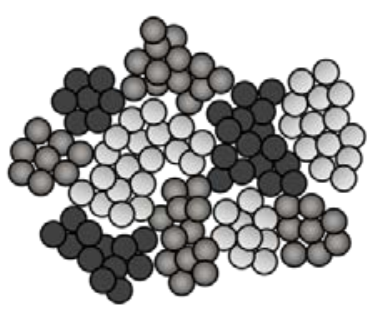

silica cluster
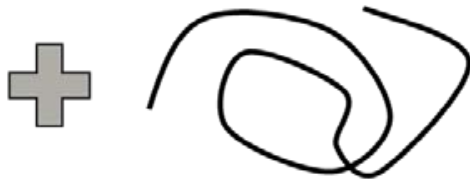

polymer

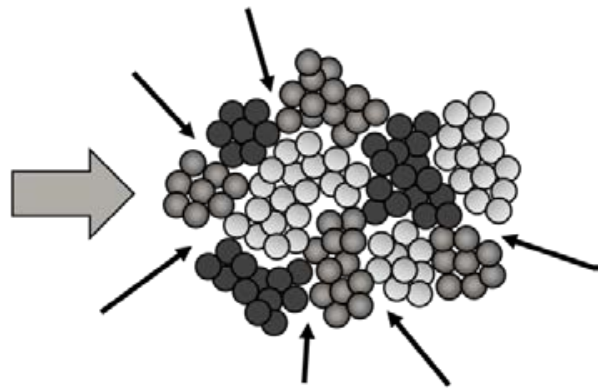

Figure 6.13: Schematic view of the dense fractal structure of silica and its initially accessible voids for the polymer

Assuming that it is possible to detect all voids of a filler by means of the void volume measurement, but only partly accessible voids are initially able to take part into the dispersion process a direct correlation between the analytical parameter and the dispersibility cannot be expected.

Finally, it is necessary to differ between the initial structure silica possesses in its original form after the production and its in-rubber structure. The latter only matters inside the final rubber product and has to be built-up during vulcanization with the use of bi-functional silanes, whereas the initial structure influences the dispersion process itself. 
Assuming that all other analytical parameters of the filler are on a constant level, a high initial structure at the beginning of the mixing process is preferred [21]. Fig. 6.14 shows the influence of different initial structures on the dispersion quality. Four different silica granules with an identical CTAB surface area and varying DBP values [22] were mixed into a green tire formulation and the dispersion quality was measured by means of the Topography Test [12].

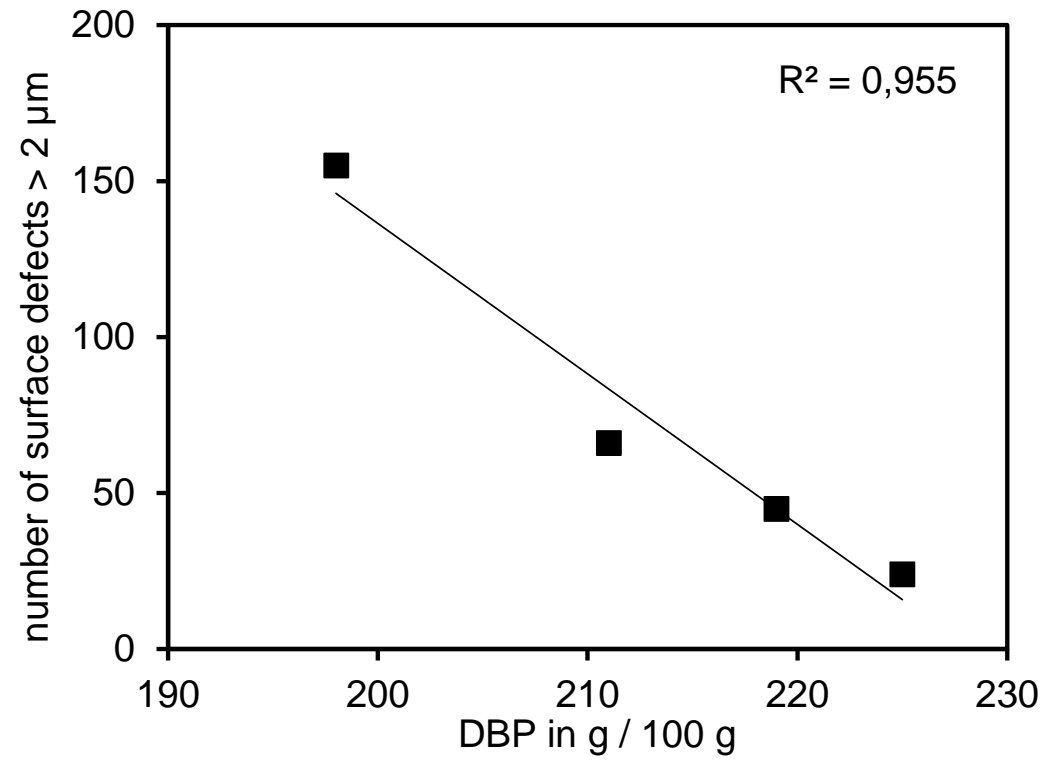

Figure 6.14: Dependency of the macro-dispersion quality of four types of silica with identical CTAB surface areas and different DBP values within a green tire formulation [12]

It can be seen that a higher DBP number respectively initial structure leads to less number of surface defects larger than $2 \mu \mathrm{m}$. A higher initial structure initially provides more possibilities for the polymer to penetrate into the accessible voids and starts the dispersion procedure. Therefore, it is easier to transmit mechanical stress/strain forces applied from the internal mixer to overcome filler-filler interactions (cohesive forces) and break down silica clusters to a smaller size. Consequently, the dispersion process is enhanced.

\subsubsection{Final Conclusion}

The results of the present chapter demonstrate that the investigated silica possesses smaller primary particles sizes, a less pronounced branching and a higher amount of void areas in comparison to carbon black as shown by the FTIR-studies and TEM and SEM images. On the one hand, the lower branching factor can explain why different types of silica cannot be distinguished with the void volume structure measurement at high pressures. The remaining higher void area in between the structure on the other hand can explain why the absolute measured end values (void volume at $125 \mathrm{MPa}$ ) are higher for silica. In addition, it was shown that the void volume structure measurement does not change the chemistry of silica.

Due to the fact that carbon black shows a hysteresis during decompression it is likely that the elasticity respectively flexibility of the structure is higher in comparison to silica. In this way, the open branches of CB are able to slide into each other during compression resulting in less free voids. The initial structure of silica, which is also dependent on the dosage form (chapter 4.2), on the contrary, seems to break down during compression resulting in a less branched structure including a high amount of free voids. Therefore, it is not possible to distinguish between different types of silica at high pressures. As a conclusion it can be said that the structure of silica differs significantly from the structure of carbon black. The latter possesses an open fractal structure whereas it is preferred to use the term "dense fractal structure" in the case of precipitated silica. 


\subsection{References}

[1] ISO 5794:2010 - Rubber compounding ingredients - Silica, precipitated, hydrated - Part 1: Non-rubber tests

[2] ISO 19246:2016 - Rubber Compounding Ingredients - Silica - Oil absorption of precipitated silica

[3] A. Blume, Operando infrared study of the reaction of triethoxypropylsilane with silica, Kautsch. Gummi Kunstst. 8 (2008) 359-362

[4] ISO 289-1:2014 - Rubber, unvulcanized - Determinations using a shearing-disc viscometer - Part 1: Determination of Mooney viscosity

[5] A. Wehmeier, "Entwicklung eines Verfahrens zur Charakterisierung der Füllstoffdispersion in Gummimischungen mittels einer Oberflächentopographie", 1998, Fachhochschule Münster, Münster

[6] ASTM D 2414:2016 - Standard Test Method for Carbon Black - Oil Absorption Number (OAN)

[7] ASTM D3493:2016 - Standard Test Method for Carbon Black - Oil Absorption Number of Compressed Sample (COAN)

[8] A. R. Payne, Effect of dispersion on dynamic properties of filler-loaded rubbers, Rubber Chem. Technol. 39 (1966) 365-374

[9] W. Niedermeier; J. Fröhlich, Moderne Füllstoffsysteme in der Gummiindustrie, presented at: Lehrstuhlseminar AG Prof. Göritz, Regensburg, Germany (2003)

[10] H.-D. Luginsland et al., Influence of Different Silanes on the Reinforcement of SilicaFilled Rubber Compounds, Rubber Chem. Technol. 75 (2002) 563-579

[11] H.-D. Luginsland, A Review on the Chemistry and the Reinforcement of the Silica-Silane Filler System for Rubber Applications, Shaker Verlag, Cologne (2002)

[12] Internal Information, Evonik Resource Efficiency GmbH

[13] R. K. Iler, The Chemistry of Silica, John Wiley \& Sons, New York - Chichester - Brisbane - Toronto (1978)

[14] M. el Roz, Infrared characterisation of Silica samples (I), Laboratoire Catalyse et Spectrochimie - ENSICAEN (2016)

[15] ASTM D7854:2016 - Standard Test Method for Carbon Black-Void Volume at Mean Pressure

[16] ASTM D6556:2016 - Standard Test Method for Carbon Black - Total and External Surface Area by Nitrogen Adsorption

[17] D. Göritz et al., Reinforcement with precipitated silica, Kautschuk Herbst Kolloquium KHK, Hanover, Germany (2004)

[18] L. A .E. M. Reuvekamp, "Reactive mixing of silica and rubber", PhD: 2003, Elastomer Technology Engineering, University of Twente, Enschede, The Netherlands

[19] ASTM D3849:2007 - Standard Test Method for Carbon Black - Morphological Characterization of Carbon Black Using Electron Microscopy

[20] P. Albers et al., Physical boundaries within aggregates - differences between amorphous, para-crystalline, and crystalline structures, Cryst. Res. Technol. 11 (2015) 846-865

[21] S. Uhrlandt and A. Blume, Kieselsäure für den Grünen Reifen - Prozesse, Produkte, Eigenschaften, Kautsch. Gummi Kunstst. 54 (2001) 520-527

[22] ASTM D6854:2012a - Standard Test Method for Silica-Oil Absorption Number 


\section{Chapter 7 - Summary}

Since the introduction of the "Green-Tire" by Michelin in 1992 precipitated silica in combination with bi-functional organosilanes became one of the most important fillers for passenger car tire tread compounds. This filler system leads, in combination with a special polymer system, to a better wet traction and lower rolling resistance in comparison to carbon black filled treads which results in a higher safety performance and a lower fuel consumption. However, up to now it is still challenging to obtain an equivalent or even improved level of abrasion resistance which would improve the service life of a tire and, in the end, would reduce the amount of scrap tires per year. This abrasion resistance of silica filled passenger car tire treads is strongly influenced by the macro-dispersion quality of the filler. The expression "macro-dispersion" characterizes the dispersion quality in the size range between 2 and $100 \mu \mathrm{m}$. A better macro-dispersion, which means less undispersed filler-particles, results in a higher abrasion resistance. To improve the macro-dispersion and therefore the abrasion resistance of passenger car tire tread compounds it is crucial to understand how the dispersion quality of silica inside a rubber compound can be improved.

Previous works showed that the compound formulation and the mixing process have a great impact on the in-rubber dispersion quality of silica. Some of the influencing parameters of the mixing process and the varying ingredients of the formulation are contradictory. The silane for instance hydrophobizes the silica surface. As a result, the filler-filler interactions are reduced and the dispersion behavior is improved. Therefore, a fast and sufficient silanization reaction is desired. A high mixing temperature and a longer mixing time can fulfill this requirement. In contrast, both mentioned mixing parameters increase the risk of polymer degradation during the mixing. Consequently, the chosen parameters for the mixing process represent always a compromise to achieve the objectives.

The analytical properties of silica itself have a great impact on the in-rubber dispersion quality as well. The higher the specific surface area is for instance, the more pronounced are fillerfiller interactions and the more difficult it is to disperse silica clusters. In contrast to the compound formulation and mixing process, the silica can be optimized regarding its dispersibility without facing any conflicts. The term "dispersibility" describes the ability of silica to be dispersed into a rubber matrix. This dispersibility can only be assessed within an identical compound formulation and mixing process and is exclusively be influenced by the fillers properties. Considering, that the dispersibility is known, it would be possible to adjust it precisely. Thus, tailor-made silica could be designed which would show an improved dispersibility. Hence, to develop new highly dispersible silica it is crucial to be aware of the typical analytical silica parameters and their impact on the dispersion process.

At the beginning of this work, no direct correlation between one single analytical silica parameter and its dispersibility was known. For that reason, it was desirable to develop a new analytical method to predict the in-rubber dispersibility of silica. This analytical method should have a proper repeatability and reproducibility and should be able to distinguish between different types of silica. To evaluate the dispersibility of different types of silica, it was essential to use an identical formulation and an identical mixing process. A variety of silica with different analytical parameters were investigated and their effect on in-rubber properties within different compounds was evaluated. Therefore, the research of this thesis is focused on the development of a new analytical method to predict the dispersibility of silica. To achieve this objective, three different analytical methods to characterize precipitated silica for tire tread applications were developed and evaluated for their suitability. Finally, a correlation analysis between analytical parameters of silica, the already known and the newly developed ones, and their effect on in-rubber properties was carried out. The final goal was to predict the dispersibility of silica by means of one single analytical parameter. 
A fundamental overview to rubber technology and relevant topics concerning this thesis are presented in chapter 2 - Literature Review. A basic understanding of the production of silica is given. The chemistry and morphology is described and the influences of analytical properties of silica on the in-rubber properties are shown. An emphasis was placed on the dispersion of silica and the dispersion quality.

In chapter 3 - Rubber Compounding and Testing, all types of silica used in this work were described by means of their five standard analytical parameters, the CTAB, BET, DOA, pHvalue and moisture content. All investigated formulations for the mixed compounds, the mixing processes and in-rubber tests to evaluate the dispersion quality of silica in different rubber formulations were described. The mixing curves and in-rubber tests were evaluated and conspicuous differences within the results were highlighted. In total, 25 different types of silica with different dosage forms were mixed into four compound series. The Green Tire compounds filled with $80 \mathrm{phr}$ of silica were defined as the reference series. In addition, another Green Tire test series with 50 phr of silica was evaluated. Moreover, NR compounds filled with 55 phr of silica were investigated. All three test series include the use of silane inside the formulation. Finally, Green Tire compounds filled with $80 \mathrm{phr}$ of silica but without using silane were mixed. The macro-dispersion qualities of all four series were evaluated by means of the Topography and Dispertester measurement systems. Furthermore, the PAYNE-effect of the rubber compounds was investigated, which is stated in the literature as an indirect measurement for the micro-dispersion quality. In case of the reference series (GT80), special investigations were conducted on selected compounds. First of all, samples were evaluated by means of the Dispergrader, a measurement system comparable to the Dispertester. In addition, the dispersion quality was measured with a confocal light microscope and with an X-ray computed microtomography $(\mu \mathrm{m}-\mathrm{CT})$. Finally, the abrasion resistance of several compounds were evaluated by means of the laboratory abrasion tester (LAT100).

The NR compounds in general showed the best dispersion qualities of all series whereas the GT50 compounds show the widest spreading of results. Surprisingly, the GT80 compounds without silane possessed slightly better dispersion results than the GT80 compounds with silane. Therefore, it was concluded that the effect of silane as a dispersion aid on the macrodispersion is less pronounced than the effect of higher shear forces during the mixing process due to a higher compound viscosity in the absence of silane. However, the PAYNE-effect could be reduced significantly by means of silane and therefore a better micro-dispersion and reinforcement can be expected.

When comparing the Dispertester and Topography measurements the GT80 as well as the NR and the GT80 series without the use of silane show a decent correlation. In this case, the two different measurement principles (mechanical and optical) can be compared to each other. A direct correlation of the outputs of the Dispertester and Dispergrader system expressed comparable results which was expected due to the fact that the principles of the measurements are similar. With the use of the confocal light microscope (CLM) it was possible to evaluate the dispersion quality of fillers over a wider size range even to a rough sub-micron level. It turned out that the use of different lenses respectively magnifications did not necessarily result in similar dispersion qualities. First results of the X-ray computed microtomography showed that it is necessary to investigate a higher amount of samples to be able to judge the repeatability before the results can be interpreted.

An inside view into the principles of an analytical method development was provided in chapter 4.1 - The Principles of Method Development and Sample Preparation. These principles were divided into four different categories: The purpose of the measurement, either for quality and production control or to support the development of new products; the requirements an analytical method has to fulfil including a proper repeatability, distinguishability of different types of silica, an independency of storages effects and dosage form and the suitability for a daily lab routine; the variation possibilities including the parameters which can be adjusted by the 
device and the sample preparation; and finally, different evaluation possibilities to compare the results.

A special emphasize was put on the sample preparation. For instance, the moisture content of silica can influence the final result as well as the dosage form and therefore both aspects have to be considered. When a reduction of particle clusters is needed, cavitation by means of ultrasound plays a major role. Various parameters can thereby influence the ultrasonic sample preparations and the wear of the sonotrode has to be taken into account as well. When using a particle size distribution measurement system it is important to be aware of the assumptions and approximations used to calculate the diameters. Results obtained by measuring different types of silica can only be compared relatively to each other and it is not be possible in general to compare absolute values from different devices with each other.

Chapter 4.2 until 4.4 include the development of three new analytical methods with different approaches to get a better insight into the dispersibility of silica.

The chapter 4.2 - Method Development 1: Void Volume Structure Measurement, presented a new analytical system to determine the initial structure of silica which is known from previous investigations to influence the dispersion process. The void volume structure measurement calculates the void volume of a filler during compression inside a piston/cylinder system. The higher the void volume is, the higher is the structure of the filler. This method shows a proper repeatability, a good differentiation between different types of silica and is independent of storage effects. The outcome of this measurement depends on the machine parameters, the sample weight and the sample preparation. Especially the moisture content of different grades of silica has to be taken into consideration when comparing the results. Therefore, it is recommended to dry all samples before the measurement occurs. The comparison of the void volume measurement evaluation with the DOA values results in a high correlation. Hence, this newly developed method has an additional benefit as being a favorable alternative to the common DOA measurement due to the fact that it performs faster and avoids the use of oil.

The dispersion process of silica throughout the rubber matrix occurs during the mixing process. To evaluate the dispersibility of silica by means of an analytical method chapter 4.3 - Method Development 2: Sedimentation Analysis, provides an attempt to transfer the dispersion process into the laboratory. For this purpose, a solution of silica and demineralized water was treated with ultrasound in order to simulate the dispersion process of silica in rubber. After this preparation, the particle size distribution of the silica in the suspension was measured by the use of a sedimentation analysis. Thereby, particles inside the suspension settles by means of gravity and are detected by an X-ray beam which is partly absorbed by the silica. The lower the amount of residual big particles is, the easier it should be to break down clusters respectively to disperse the silica.

The sedimentation analysis depends on the sample preparation, used concentration of silica in water and the X-ray intensity. Additionally, a dependency due to storage was deter-mined which has to be taken into consideration when measuring silica samples. The strongest influence occurred due to the use of ultrasound as a sample preparation method. Especially the wear of the sonotrode has to be monitored in order to avoid deviations of results over a period of time. A suitable reproducibility was achieved and it was shown that it is possible to distinguish between different types of silica.

During mixing of silica inside a rubber matrix, the particle size of the filler is successively reduced which is referred to as dispersion process. Some types of silica are easier to decrease in their cluster sizes than others. To gain information about the easiness and speed of the cluster breakage inside the rubber matrix an approach was made in chapter 4.4-Method Development 3: In-situ Cluster Fragmentation, to observe this size reduction during a defined energy input on lab scale. For this purpose, a silica suspension circulates in a closed system with controlled velocity speed. During each circulation, the suspension passes an ultrasonic 
device to reduce the cluster size and a laser diffraction system to measure the particle size distribution. The faster different curve characteristics such as the mean degradation rate and Dv(50) value changes over time, the easier the breakdown of bigger clusters should be. This new method was named "in-situ cluster fragmentation". It shows a sufficient repeatability and enables the differentiation of different types of silica. The final result depends mainly on the chosen conditions of the ultrasonic treatment. Varying the sample mass, flow velocity and temperature of the suspension have a minor impact. A certain influence due to storage conditions could be observed and the wear of the sonotrode has to be monitored.

In chapter 5 - Correlation between Analytical Parameter and In-rubber Dispersion Quality, the correlation trials between the five standard analytical parameters described in chapter 3 of all types of used silica and the measured macro-dispersion results are presented. Moreover, the different evaluation parameters obtained by three newly developed analytical methods: the void volume structure measurement (chapter 4.2), the sedimentation method (chapter 4.3) and the in-situ cluster fragmentation (chapter 4.4) were correlated to these macro-dispersion results as well.

The only relation found to the standard analytical parameters was that the PAYNE-effect results of the GT80 series without the use of silane correlate to the surface area (CTAB and $B E T)$, whereas all macro-dispersion measurements do not show any sufficient correlation. Therefore, it was concluded that the specific surface area only has an influence on the microbut not on the macro-dispersion quality. The higher the specific surface area is, the more pronounced are filler-filler interactions respectively a filler network what can explain a higher PAYNE-effect. The void volume structure measurement does not show any relation to the level of dispersion at all, whereas the sedimentation method and the in-situ cluster fragmentation provides certain indications to predict the dispersibility of a silica. However, it was not possible to predict the in-rubber dispersibility of silica completely sufficiently with these methods. The indications provided by the sedimentation method and in-situ cluster fragmentation are nevertheless a good approach and therefore useful to characterize silica and support the development of new products.

Additional investigations with the Dispergrader and a confocal light microscope (CLM) did not provide an improvement of the correlation coefficients in comparison to previous measurements. Furthermore, the abrasion resistance of eleven selected rubber compounds of the GT80 series with silane were measured by means of the Laboratory Abrasion Tester (LAT100) and correlated to the macro-dispersion results. It was expected that a better macro-dispersion quality results in an improved abrasion resistance but no correlation between these parameters could be found. However, a correlation to the PAYNE-effect was obtained which indicates a relation to the micro-dispersion quality. Due to the fact that the influence of the silane on the PAYNE-effect was shown beforehand it can be assumed that the presence of silane directly influences the abrasion resistance.

As a final attempt, a data analyzing model (Partial Least Squares - PLS) was used to check if a combination of different parameters can predict the in-rubber dispersibility more sufficiently. A final equation containing several parameters provides an improved indication compared to the single linear correlation. Especially the outcome of the sedimentation analysis and the insitu cluster fragmentation are of higher importance for the calculation. In order to evaluate more reliable results a higher amount of data sets is needed.

Chapter 6 - Discussion and Conclusion - Investigation of the Morphology of Silica, summarizes different aspects, which limit the general approach to predict the in-rubber dispersibility of silica in a very accurate way. One reason e.g. was the compromise made to adjust the amount of silane to the specific CTAB surface area of different types of silica to ensure a consistent level of hydrophobation. In this way, the viscosity slightly varied from compound to compound which effected the dispersion process. But not adjusting the amount of silane to the surface area would lead to an insufficient hydrophobation when using too less silane and as a 
consequence to pronounced filler-filler interactions. With both approaches a certain mistake is made which has to be taken into account. Therefore, different obtained dispersion qualities are not exclusively affected by the properties of silica itself. Another reason why it is not possible to sufficiently predict the in-rubber dispersibility of silica by means of the sedimentation method and in-situ cluster fragmentation is the fact that it is impossible to directly transfer the dispersion process into the laboratory. One the one hand, silica is dispersed inside a high viscous rubber matrix in an internal mixer including different other ingredients like processing oil which influence the dispersion process as well. On the other hand, the silica is simply dispersed inside water in both laboratory approaches to obtain a suspension which is treated by means of ultrasound. Both processes strongly differ from each other and can therefore not be easily compared. As a conclusion, it became obvious that a linear correlation of one single analytical parameter with the macro-dispersion quality cannot be found.

Furthermore, open questions arose from the void volume structure measurement were discussed. This method was originally developed to investigate the structure of carbon black. Especially the comparable remaining high void volume of silica at $125 \mathrm{MPa}$ as well as the fact that all types of silica converge at higher pressures and hence cannot be distinguished anymore were investigated. On the contrary, carbon black possess different values at $125 \mathrm{MPa}$ depending on their structure. Therefore, it was presumed that the structure of silica and black strongly differ from each other and cannot be compared. To confirm differences between both types of filler structures and to investigate changes within the chemistry and morphology of silica during the void volume measurement, X-ray diffraction measurements, FTIR-studies, TEM and SEM images were conducted by measuring selected silica samples, in the virgin status and after being compressed by the void volume measurement.

The final conclusion is that the structure of both types of fillers differ significantly. Carbon Black possesses on the one hand an open fractal structure whereas precipitated rubber silica on the other hand exhibits a dense fractal structure. For the latter a distinction has to be made between the initial structure after production and the in-rubber structure which is build-up during vulcanization by means of bi-functional silanes.

The aim of the present work was to predict the in-rubber dispersibility of silica by means of one single analytical parameter. Therefore, standard analytical parameters of silica as well as parameters obtained by newly developed methods were investigated and correlated to the inrubber macro-dispersion quality. It turned out that many different factors influence this direct correlation which makes it more difficult to predict the dispersibility sufficiently. By using a data analyzing model, a combination of different analytical parameters could be found which provides an improved indication compared to the single linear correlation. Moreover, by means of the newly developed methods it was possible to get a better insight into the dispersibility of silica and to get a deeper understanding of the morphology of silica. 


\section{Samenvatting}

Sinds de introductie van de zogenaamde "groene" autoband door Michelin in 1992 is silica in combinatie met bifunctionele silanen een van de meest belangrijke vulstoffen in autobandenloopvlakken mengsels. Dit vulstof-systeem leidt in combinatie met een speciaal polymeer systeem tot een betere grip op natte wegen en een lagere rolweerstand ten opzichte van loopvlakken die als vulstof roet bezitten . Dit resulteert in een hogere veiligheid en een vermindering van brandstofverbruik. Tot nu toe is het nog steeds een uitdaging om een gelijke of betere slijtage weerstand te bereiken, waardoor de levensduur van de banden wordt verbeterd en uiteindelijk het aandeel van gebruikte banden per jaar wordt gereduceerd.

De slijtage weerstand van autobanden loopvlakken met silica wordt sterk beïnvloedt door de macro-dispersiekwaliteit van de vulstof. De uitdrukking "macro-dispersie" beschrijft de dispersiekwaliteit tussen 2 en $100 \mu \mathrm{m}$. Een betere macro-dispersie, welk overeenkomt met minder slecht gedispergeerde vulstof deeltjes, resulteert in een betere slijtage weerstand. Om de macro-dispersie te verbeteren en daarmee ook de slijtage weerstand van autobandenloopvlakken mengsels is het belangrijk om te begrijpen hoe de dispersiekwaliteit van silica in rubber mengsels kan worden verbeterd.

Voorgaand onderzoek heeft aangetoond dat de mengselsamenstelling en het mengproces een grote invloed op de dispersiekwaliteit van silica in rubber hebben. Enkele van de invloedrijke parameters van het mengproces en van de variërende ingrediënten van de rubber samenstelling zijn tegenstrijdig. Het silaan bijvoorbeeld, hydrofobeert het oppervlak van de silica, welk resulteert in een gereduceerde vulstof-vulstof interactie en een betere dispersie. Om deze reden is een snelle en efficiënte reactie van het silaan nodig. Een hoge mengtemperatuur en een lange mengtijd kunnen aan de eisen voldoen, maar beide mengparameters verhogen tevens het risico op polymeer degradatie tijdens het mengproces. De consequentie is dat de gekozen parameters voor het mengproces altijd een compromis moeten zijn om de gewenste eigenschappen te verkrijgen.

De analytische eigenschappen van silica hebben ook een grote invloed op de dispersiekwaliteit. Hoe hoger bijvoorbeeld de specifieke oppervlakte is, hoe groter het aandeel aan vulstof-vulstof interacties is des te moeilijker is het de dispersie van de silica clusters te bewerkstelligen. In tegenstelling tot de mengselsamenstelling en het mengproces, kan de silica worden geoptimaliseerd met betrekking tot haar "dispergeerbaarheid" zonder andere compromissen te sluiten. De uitdrukking "dispergeerbaarheid" beschrijft hoe goed de silica in de rubber kan worden verdeeld. De dispergeerbaarheid kan alleen maar worden bepaald in een identieke mengselsamenstelling en mengproces en wordt enkel beïnvloed door de eigenschappen van de vulstof. Als de dispergeerbaarheid op voorhand gemeten kan worden, dan zou het mogelijk zijn om deze naar specifieke wensen aan te passen; Met andere woorden, dit zou het mogelijk maken om silica te produceren die exact is afgestemd op de eisen van de industrie met een verbeterde dispergeerbaarheid. Om nieuwe silica met een verbeterde dispersie te kunnen ontwikkelen, is het cruciaal om de typische analytische parameters van de silica te kennen en de invloed van deze parameters op het dispersieproces.

Aan het begin van dit onderzoek waren geen directe correlaties tussen een analytische parameter van de silica en haar dispergeerbaarheid bekend. Om deze reden was het gewenst een nieuwe analytische methode te ontwikkelen om de dispergeerbaarheid van silica in rubber te voorspellen. Deze analytische methode zou een goede herhaalbaarheid en reproduceerbaarheid moeten hebben en zou verschillende typen silica van elkaar moeten kunnen onderscheiden. Om de dispergeerbaarheid van verschillende types silica te beoordelen, is het van groot belang om een identieke mengselsamenstelling en een identiek mengproces te gebruiken. 
Een groot aantal verschillende typen silica met verschillende analytische eigenschappen werd onderzocht met betrekking tot het effect op de rubber eigenschappen van verschillende mengselsamenstellingen. Het onderzoek van dit proefschrift richt zich op de ontwikkeling van een nieuwe analytische methode om de dispergeerbaarheid van silica te voorspellen. Om dit doel te bereiken zijn drie verschillende analytische methoden ontwikkeld en beoordeeld om silica te karakteriseren voor het gebruik in autobanden loopvlakken. Ten slotte is er een correlatie analyse uitgevoerd tussen de analytische parameters van de silica, de standaard analytische parameters en de nieuw ontwikkelde parameters, en hun effect op de rubber eigenschappen. Het uiteindelijke doel was de dispergeerbaarheid van silica in rubber alleen met een analytische parameter te voorspellen.

Een fundamenteel overzicht over de rubber technologie en verder belangrijke onderwerpen voor dit proefschrift worden gepresenteerd in hoofdstuk 2 - literatuur overzicht. Een basisbegrip van de productie van silica wordt gegeven. De chemie en de morfologie van silica worden beschreven en de invloed van de analytische eigenschappen van silica op de rubber eigenschappen wordt getoond. De nadruk van dit onderzoek ligt op de dispersie van silica en de dispersiekwaliteit.

In hoofdstuk 3 - rubber compounding en tests - werden alle typen silica die in dit proefschrift worden gebruikt beschreven met hun vijf standard analytische parameters: CTAB, BET, DOA, $\mathrm{pH}$ en vochtigheid. Alle onderzochte mengselsamenstellingen, het mengproces en de diverse meetmethoden om de dispersiekwaliteit van silica in verschillende rubber samenstellingen te beschrijven werden voorgesteld. De mengcurven en resultaten uit de diverse meetmethoden werden geëvalueerd en interessante verschillen van de resultaten werden benadrukt. In totaal werden 25 verschillende typen van silica met verschillende doseervormen in vier series gemengd met verschillende mengselsamenstellingen. De "groene" autoband samenstelling met $80 \mathrm{phr}$ silica werd als de referentie gedefinieerd. Verder werd een andere "groene" autoband samenstelling met 50 phr silica onderzocht.

Tevens werden er NR compounds met 55 phr silica getest. Bij alle drie series werd silaan gebruikt. Daarnaast werd er ook een "groene" autoband samenstelling met 80 phr silica, maar zonder silaan gemengd. De macro-dispersiekwaliteit van deze vier series werd geëvalueerd met behulp van Topografische en Dispertester meetsystemen. Bovendien werd het PAYNEeffect van de rubber mengsels onderzocht, dat in de literatuur als een indirect indicator van micro-dispersie wordt genoemd. Voor de referentieserie GT80 werd verder onderzoek met specifiek geselecteerde monsters uitgevoerd. De eerste methode was de Dispergrader, een meetsysteem dat met de Dispertester vergelijkbaar is. Verder werd de dispersiekwaliteit met een confocale licht microscoop en met een röntgen computer tomografie $(\mu \mathrm{m}-\mathrm{CT})$ onderzocht. Tot slot werd nog de slijtage weerstand van verschillende mengsels met het slijtage weerstand apparaat (LAT100) gemeten.

De NR compounds lieten in het algemeen de beste dispersiekwaliteiten van alle series zien, terwijl de GT50 compounds de grootste spreiding in de resultaten weergaven. Verrassend genoeg hadden de GT80 compounds zonder silaan een beter dispersieresultaat dan de GT80 compounds met silaan. Vandaar werd de conclusie getrokken dat het effect van silaan als dispersie hulpstof op de macro-dispersie minder belangrijk is dan het effect van hogere afschuifkrachten tijdens het mengproces vanwege een hogere compound viscositeit zonder silaan. Het Payne-effect kon significant worden gereduceerd door het silaan, waardoor een beter micro-dispersie en versterking kan worden verwacht.

Wanneer de resultaten van de Dispertester en de Topografische metingen worden vergeleken van de GT80 compounds en ook van de NR en GT80 compounds zonder silaan, wordt een goede correlatie duidelijk. In dit geval kunnen deze twee verschillende meetmethoden (mechanisch en optisch) worden vergeleken. Er werd een directe correlatie tussen de resultaten van de Dispergrader en de Dispertester vastgesteld; Dit werd verwacht aangezien de principes van de metingen op elkaar lijken. 
Met behulp van de confocale licht microscoop (CLM) was het mogelijk de dispersiekwaliteit van vulstoffen over een bredere orde van grootte tot het sub-micro niveau te meten. Het bleek dat de verschillende lenzen en daarmee ook de vergrotingen niet tot hetzelfde resultaat leidden. De eerste resultaten van de röntgen computer tomografie tonen aan dat het noodzakelijk is een groter aantal aan monsters te onderzoeken om de reproduceerbaarheid te kunnen beoordelen voordat de resultaten kunnen worden geëvalueerd.

Een inzicht in de principes van een analytische methode ontwikkeling is gegeven in hoofdstuk 4.1 - de principes van methode ontwikkeling en monsterpreparatie. Deze principes worden onderscheiden in vier verschillende categorieën: het doel van de meting, of voor kwaliteit en productiecontrole of om de ontwikkeling van nieuwe producten te ondersteunen; De analytische methode moet een goede reproduceerbaarheid hebben, en onderscheid kunnen maken tussen verschillende types silica, moet onafhankelijk van de doseervorm van de silica zijn en geschikt zijn voor een dagelijks laboratorium routine; de mogelijke variaties samen met de machineparameters van de apparatuur die aangepast kunnen worden en de monsterpreparatie; Tot slot de verschillende evaluatie mogelijkheden om de resultaten te vergelijken.

Er werd veel aandacht besteed aan de monsterpreparatie. Bijvoorbeeld, de vochtigheid van silica kan het definitieve resultaat beïnvloeden net zoals de silica doseervorm en daarom moeten beide aspecten worden overwogen. Indien een reductie van de deeltjesgrootte nodig is, speelt cavitatie doormiddel van ultrasoon geluid een belangrijke rol. Verschillende parameters kunnen de monsterpreparatie met behulp van ultrasoon geluid beïnvloeden en daarnaast dient ook de slijtage van de sonotrode in acht te worden genomen. Als een meetsysteem voor deeltjesgrootteverdeling wordt gebruikt, is het erg belangrijk om de aannamen en de benaderingen voor de berekeningen van de diameters te kennen. De resultaten van metingen van verschillende typen van silica kunnen allen maar relatief met elkaar worden vergeleken. Het is niet mogelijk in het algemeen om absolute waarden van verschillende apparatuur met elkaar te vergelijken.

Hoofdstuk 4.2 tot 4.4 beschrijven de ontwikkeling van drie verschillende nieuwe analytische meetmethoden met verschillende benaderingen om een beter inzicht van de dispergeerbaarheid van silica te verkrijgen.

In hoofdstuk 4.2 - methode ontwikkeling 1: Leeg Volume Structuur Meting, wordt een nieuw analytisch system gepresenteerd om de initiële structuur van silica te bepalen omdat uit eerdere onderzoeken is gebleken dat dit een invloed op het dispersieproces heeft. De leeg volume structuur meting berekent het leeg volume van een vulstof tijdens de compressie in een piston / cilinder systeem. Hoe hoger het leeg volume is, des te hoger is de structuur van de vulstof. Deze methode toont een goede herhaalbaarheid, een goede onderscheidbaarheid tussen verschillende typen silica en is onafhankelijk van opslageffecten. Het resultaat van de meting is afhankelijk van de parameters van de apparatuur, het gewicht van het monster en de monsterpreparatie. In het bijzonder, dient er rekening te moeten worden gehouden met de vochtigheid van de verschillende typen silica bij de vergelijking van de resultaten. Daarom is het aanbevolen alle monsters te drogen voordat de meting wordt uitgevoerd. Het vergelijk van de leeg volume structuur meting evaluatie tezamen met de DOA waarden resulteert in een goede correlatie. Deze nieuw ontwikkelde meetmethode heeft als voordeel dat deze sneller is en maakt in tegenstelling tot de DOA methode geen gebruik van olie; De nieuwe meetmethode verdient dan ook de voorkeur boven de DOA methode.

Het dispersieproces van silica in de rubber matrix vindt plaats tijdens het mengproces. Om de dispergeerbaarheid van silica door een analytische methode te beschrijven, wordt in hoofdstuk 4.3 - methode ontwikkeling 2: Sedimentatie Analyse, getracht om het dispersieproces in het laboratorium te simuleren. Om deze reden wordt een oplossing van silica en gedemineraliseerd water met ultrasoon geluid behandeld om het dispersieproces van silica in rubber na te bootsen. Na deze voorbereidingen wordt de deeltjesgrootteverdeling van silica in de suspensie met hulp van een sedimentatie analyse gemeten. 
Hierbij zetten zich deeltjes uit de suspensie af vanwege de zwaartekracht. Deze deeltjes worden gedetecteerd door een röntgenstraal die voor een deel door de silica wordt geabsorbeerd. Hoe lager het aandeel van resterende grote deeltjes is, des te makkelijker is het om de silica clusters te verbreken en des te eenvoudiger zal de silica zich laten dispergeren.

De sedimentatie analyse is afhankelijk van de monsterpreparatie, de concentratie van de silica in water en van de röntgen intensiteit. Verder werd er waargenomen dat de opslagcondities van invloed zijn op de meetresultaten; Hiermee dient rekening gehouden te worden als silica volgens deze methode wordt gemeten. De grootste invloed komt doordat ultrasoon geluid als monsterpreparatie methode gebruikt wordt. Met name de slijtage van de sonotrode moet goed worden gecontroleerd om afwijkingen van resultaten in loop van de tijd te vermijden. Een goede reproduceerbaarheid werd bereikt en er werd getoond dat het mogelijk is om verschillende types silica te onderscheiden.

Tijdens het mengen van silica in rubber wordt de deeltjesgrootte van de vulstof successief gereduceerd, hetgeen het dispersieproces wordt genoemd. Enkele types van silica kunnen makkelijker worden gereduceerd qua deeltjesgrootte dan andere. Om informatie met betrekking tot de eenvoud en de snelheid van de silica clusterbreuk in de rubber matrix te krijgen, wordt in hoofdstuk 4.4 - methode ontwikkeling 3: In-situ Cluster Fragmentatie, getracht om deze deeltjesgrootte reductie tijdens een gedefinieerde energie input op labschaal te bestuderen. Om dit te bewerkstelligen werd een silica suspensie gecirculeerd in een gesloten systeem middels een gecontroleerde snelheid. Voor elke cyclus tijdens de circulatie komt de suspensie aan de ultrasone voorbij om de cluster grootte te reduceren. De deeltjesgrootteverdeling wordt gemeten met een laserdiffractie systeem.

Hoe sneller verschillende karakteristieke eigenschapen, zoals de "gemiddelde afbraaksnelheden" en de "Dv(50)" waarden, zich met de tijd veranderen, des te makkelijker zou de afbraak van grote silica clusters moeten zijn. Deze nieuwe methode wordt de "in-situ cluster fragmentatie" genoemd. De methode toont een goede herhaalbaarheid en maakt het mogelijk om verschillende types silica te onderscheiden. Het uiteindelijke resultaat hangt vooral van de gekozen voorwaarden van de ultrasone behandeling af. Variatie van monstergewicht, stromingssnelheid en de temperatuur van de suspensie hebben minder invloed. Verdere invloed is afkomstig van de opslagcondities. Verder dient de slijtage van de sonotrode te worden gemonitord.

In hoofdstuk 5 - correlatie tussen analytische parameter en rubber dispersiekwaliteit, wordt het correlatie onderzoek tussen de vijf standaard analytische parameters (zoals beschreven in hoofdstuk 3) van alle types silica en de gemeten macro-dispersie resultaten gepresenteerd. Verder worden de verschillende evaluatie parameters, afkomstig van de drie nieuwe ontwikkelde meetmethoden: de leeg volume structuur meting (hoofdstuk 4.2), sedimentatie methode (hoofdstuk 4.3) en de in-situ clusterfragmentatie (hoofdstuk 4.4, ook gecorreleerd met de betreffende macro-dispersie resultaten.

De enige relatie die werd gevonden met de standard analytische eigenschappen was tussen de PAYNE-effect resultaten van de GT80 serie zonder het gebruik van silaan met de oppervlakte (CTAB en BET); Alle anderen macro-dispersie metingen gaven geen goede correlatie met de specifieke oppervlakte van silica. Vandaar de conclusie dat de specifieke oppervlakte allen maar invloed heeft op de micro-dispersiekwaliteit, maar niet op de macro-dispersiekwaliteit. Hoe hoger de specifieke oppervlakte is, hoe meer vulstof-vulstof interacties er aanwezig zijn wat leidt tot een sterker vulstof netwerk, welke mogelijk het hogere PAYNEeffecten verklaren. De leeg volume structuur meting toont geen enkele relatie met het niveau van de dispersie aan, maar de sedimentatie methode en ook de in-situ clusterfragmentatie geven zekere indicaties om de dispergeerbaarheid van silica te voorspellen. Het was niet mogelijk om de dispersie van silica in rubber met deze methoden te voorspellen, echter zijn de indicaties van de sedimentatie methode en de in-situ clusterfragmentatie wel toereikend om de silica verder te karakteriseren en de ontwikkeling van nieuwe producten te ondersteunen. 
Aanvullende onderzoeken met de Dispergrader en een confocale licht microscoop (CLM) leveren geen verdere verbetering van de correlatiecoëfficiënten in vergelijking met de andere metingen op. Verder werd de slijtage weerstand van elf gekozen rubber monsters (van de GT80 serie met silaan) met de laboratorium slijtage weerstand tester (LAT100) gemeten en werd deze resultaten gecorreleerd met de macro-dispersie resultaten. Er werd verwacht dat monsters met een beter kwaliteit van de macro-dispersie ook een verbeterde slijtage weerstand zouden laten zien, maar er werd geen correlatie tussen deze parameters gevonden. Wel werd er een correlatie met het PAYNE-effect verkregen welke impliceert dat er een relatie is tussen de slijtage weerstand en de micro-dispersie kwaliteit. Aangezien de invloed van de silaan op de PAYNE-effect reeds werd aangetoond, is het mogelijk dat de aanwezigheid van silaan een directe invloed op de slijtage weerstand kan hebben.

Als een laatste poging werd een gegevensanalyse model (Partial Least Squares - PLS) ingezet om te zien of de combinatie van verschillende parameters de rubber dispergeerbaarheid beter kan voorspellen dan een enkele lineaire correlatie. De uiteindelijk verkregen vergelijking met enkele parameters kan een verbeterde indicatie geven in vergelijking met een enkele lineaire correlatie. Vooral de resultaten van de sedimentatie analyse en de in-situ clusterfragmentatie zijn uiterst belangrijk voor de berekening. Om meer betrouwbare data te kunnen evalueren zijn verdere datasets noodzakelijk.

Hoofdstuk 6 - discussie en conclusie - onderzoek van de morfologie van silica, vat verschillende aspecten samen die de algemene aanpak beperkt om de rubber dispergeerbaarheid van silica op een nauwkeurige manier te voorspellen. Een reden hiervoor was bijvoorbeeld de compromis om de hoeveelheid van silaan aan de specifieke CTAB oppervlakte van verschillende types van silica aan te passen om daardoor een consistent niveau van hydrofobering te verkrijgen. Op deze manier ontstaan er kleine verschillen in viscositeit tussen de mengsels welke het dispersieproces beïnvloedt. Echter als de hoeveelheid van het silaan niet wordt aangepast aan het silica oppervlak zou er onvoldoende hydrofobering plaats vinden, omdat er te weinig silaan is gebruikt, welke resulteert in een grotere vulstof-vulstof interactie.

Beide methoden leiden tot een zekere fout waarmee rekening gehouden moet worden. Dispersiekwaliteiten die op verschillende manieren worden bereikt, worden niet alleen maar beïnvloed door de eigenschappen van de silica. Een andere reden waarom het niet mogelijk is om een goede voorspelling van de dispergeerbaarheid van silica in rubber te verkrijgen met behulp van de sedimentatie analyse en de in-situ clusterfragmentatie, is het feit dat het niet mogelijk is het dispersieproces exact na te bootsen in het laboratorium. Enerzijds is silica gedispergeerd binnen een hoge viskeuze rubber matrix in een interne menger met andere ingrediënten, zoals bijvoorbeeld olie, die ook het dispersieproces kunnen beïnvloeden. Anderzijds is silica alleen maar in water gedispergeerd in beide laboratorium methoden om een suspensie te bereiken middels ultrasoon geluid. Beide processen verschillen erg van elkaar en kunnen daarom niet gemakkelijk worden vergeleken. De conclusie is dat een lineaire correlatie van een analytische parameter niet alleen maar met de macro-dispersiekwaliteit kan worden gevonden.

Verder werden er open vragen omtrent de leeg volume meting gediscussieerd. Deze methode werd oorspronkelijk ontwikkeld om de structuur van roet te onderzoeken. In het bijzonder het feit dat het resterende leeg volume van de silica bij $125 \mathrm{MPa}$ heel hoog is en dat verschillende types van silica bij hoge drukken niet meer te onderscheiden zijn, werd verder onderzocht. In tegenstelling tot silica toont carbon black verschillende waarden bij $125 \mathrm{MPa}$ afhankelijk van de structuur. Daarom werd er aangenomen dat de structuren van silica en carbon black aanmerkelijk van elkaar verschillen en niet met elkaar kunnen worden vergeleken. Om de verschillen in structuur van beide type vulstoffen te bevestigen en om te bepalen of er chemische of morfologische veranderingen plaats vinden gedurende de leeg volume test, werden extra metingen verricht; Deze metingen bestonden uit röntgen diffractie metingen, FTIR studies, TEM en SEM beelden. 
De metingen werden verricht aan gekozen silica monsters zowel voor als na de leeg volume behandeling.

De uiteindelijke conclusie is dat de structuur van beide type vulstoffen significant verschillend is. Roet heeft een geopend fractale structuur terwijl geprecipiteerde silica een dichte fractale structuur heeft. Voor silica moet ook nog een verschil worden gemaakt tussen de initiële structuur direct na productie en de rubber structuur die zich tijdens de vulkanisatie met behulp van de bi-functionele silanen vormt.

Het doel van dit proefschrift was het voorspellen van de dispergeerbaarheid van silica in rubber met behulp van een enkele analytische parameter. Daarom werden standaard analytische parameters van de silica en ook parameters, die met behulp van nieuwe methoden werden bereikt, onderzocht en met de macro-dispersiekwaliteit gecorreleerd. Uit het onderzoek bleek dat veel verschillende factoren deze directe correlatie beïnvloeden wat een voorspelling van de dispergeerbaarheid niet mogelijk maakte. Met hulp van een gegevensanalyse model kon een combinatie van verschillende analytische parameters worden gevonden die een betere indicatie gaf dan een enkele lineaire correlatie. Middels de nieuwe ontwikkelde methoden is er meer inzicht verkregen over de dispergeerbaarheid van silica en is er veel meer kennis vergaard met betrekking tot de morfologie van silica. 


\section{Bibliography}

\section{Journal Articles}

1. F. Grunert, A. Wehmeier, W. K. Dierkes, A. Blume; Void Volume Measurement - An Alternative Approach to Determine the Initial Structure of Silica; Kautsch. Gummi Kunstst., 71 (2018) 70-74

2. F. Grunert, A. Wehmeier, W. K. Dierkes, A. Blume; New Insights into the Dispersibility of Silica by Reconsidering the Filler's Morphology; Rubber Chem. Technol., submitted (2018)

3. F. Grunert, A. Wehmeier, W. K. Dierkes, A. Blume; Comparison and Evaluation of Different Analytical Methods to Predict the In-Rubber Dispersibility of Silica; Kautsch. Gummi Kunstst., submitted and accepted

\section{Presentations}

1. F. Grunert; Silica Dispersion - Influencing Parameters; $108^{\text {th }}$ Meeting of the German Rubber Society DKG Regional Group West, Bad Neuenahr-Ahrweiler, Germany, September 19-20, 2016

2. F. Grunert, A. Wehmeier, A. Blume; Prediction of In-Rubber Dispersibility of Silica by Analytical Methods; $12^{\text {th }}$ Fall - Rubber - Colloquium, Hanover, Germany, November 22-24, 2016

3. F. Grunert; Analytische Untersuchung von Kieselsäure mittels neuartiger Methoden; $22^{\text {th }}$ Meeting of the German Rubber Society DKG Regional Group Nord, Hanover, Germany, May 112017

4. F. Grunert; Prediction of In-Rubber Dispersibility of Silica by Analytical Methods; DWI | RWTH Aachen University Summer School, Aachen, Germany, July 7, 2017

5. F. Grunert, A. Wehmeier, W. K. Dierkes, A. Blume; Prediction of In-Rubber Dispersibility of Silica by Analytical Methods; International Elastomer Conference, Cleveland / Ohio, US, October 9-12, 2017

6. F. Grunert, A. Wehmeier, W. K. Dierkes, A. Blume; Prediction of In-Rubber Dispersibility of Silica by Analytical Methods; Tire Technology Expo 2018, Hanover, Germany, February $20-22,2018$

7. F. Grunert, A. Wehmeier, W. K. Dierkes, A. Blume; Development of a Sedimentation Analysis to Predict the In-rubber Dispersibility of Silica; $111^{\text {th }}$ Meeting of the German Rubber Society DKG Regional Group West, Bad Neuenahr-Ahrweiler, Germany, May 78, 2018

8. F. Grunert, A. Wehmeier, W. K. Dierkes, A. Blume; Comparison and Evaluation of Different Analytical Methods to Predict the In-Rubber Dispersibility of Silica; $13^{\text {th }}$ Fall - Rubber - Colloquium, Hanover, Germany, November 6-8, 2018 


\section{Acknowledgement}

To carry out and write this thesis was up to now by far the biggest and toughest challenge of my life. A steep and stony path paved with struggle, self-doubt and anxiety, but also with great experiences I do not want to miss in my life. In the past three years I didn't only improve on a professional working level but more important got to learn about and develop myself as a person to an extent I never could have imagined. All I achieved could not have happened without the wonderful people surrounding me. While starting to write this acknowledgement I realized the stunning and overwhelming amount of persons which accompanied me during this period of life and who truly deserve my gratitude. It's hardly possible to thank all of you but I do try my very best to mention you in the following lines:

First of all, I would like to express my gratitude to Evonik and respectively to Dr. Jens Kiesewetter for the given chance und trust to conduct this work. The financial and moreover scientific support provided the basis for the success of this joint project. My dear former colleagues in Wesseling. Thank you very much for the past three years in the Applied Technology. From day one I was welcome with open arms and hearts, treated with respect and kindness. Vielen Dank Hannes, Sankar, Olli, Andrea, Elke, Didier, und Minh. Danke euch allen aus der Physik und Mischerei, Helga, Uwe, Sabrina, Kay, Dennis, Herrn Joepen, Herrn Meineke, Michael, Guido und Kollegen. An die Mitarbeiter aus der Analytik: Liebe Hilde, Beate, Vera, Claudia, Melanie, Carina, Steffi, Sabrina, Herr Rahnenführer, Herr Erll, Jörg, Peter, Wolfgang, Thorsten und alle weiteren: Danke für diese großartige Zeit. Auch gilt mein Dank den Kollegen aus der Forschung, Dominik, Rainer, Christian, Adam und Agnieszka. Nicht vergessen möchte ich auch Timo, Frau Kaffenberger, Herrn Dr. Albers und Kollegen, die mich bei verschiedensten Aufgaben unterstützt haben.

Liebe Olga, das gegenseitige „Aufziehen“ war eine Bereicherung im Arbeitsalltag und wird schmerzlich vermisst. Lieber Karl, Danke für deine aufmunternden Worte, wenn meine Laune einmal wieder im Keller war. Lieber Dr. Heinz, ich weiß es sehr zu schätzen, dass es Ihnen, auch oder gerade speziell weil Sie ein Physiker sind, immer gelungen ist, ein offenes Ohr und eine offene Tür für mich zu haben. Lieber Frank, du schuldest mir noch einen Abend in Köln. Dörte, vielen Dank für die herzliche und offene Kölsche Art, die ich durch dich erleben durfte. Lieber Andre, ich möchte dir insbesondere für alles danken, was du für mich auf der zwischenmenschlichen Ebene geleistet hast. Die Kleinigkeiten abseits des Arbeitsalltages haben mir das Leben ungemein erleichtert. Lieber Tim, lieber Patrick, alles Gute für eure berufliche Zukunft und lasst ab und an mal von euch hören. Nadine, ich wünsche dir von Herzen all das Glück und die Freude, die du so sehr verdienst, Danke für alles. Inge, mein Hase, einen Bazi als Unterstützung im Geiste umgeben von all diesen „Norddeutschen“ war Balsam für meine Seele. Zu guter Letzt: Lieber Thomas, ich bin überglücklich und froh, das Büro und unzählige Diskussion mit dir geteilt haben zu dürfen. Aber noch viel wichtiger, du hast dich in dieser Zeit um mich gekümmert wie um einen kleinen Bruder und unsere Freundschaft ist mit das Beste, das aus diesen letzten drei Jahren in Köln entstanden ist. Dafür werde ich dir immer unendlich dankbar sein! Ihr alle seid jederzeit herzlich willkommen mich in Enschede, oder wo auch immer mein Weg mich in Zukunft hinführen wird, besuchen zu kommen!

I do want to express my gratitude to Prof. Dr. Ir. J. E. ten Elshof, to Prof. Dr. Dariusz Bieliński and to Dr. Joachim Bertrand for being part of my defense committee. Thank you very much for supporting me in this final important stage of my education. Lieber Herr Herrmann: Im Grunde könnte man Sie für all das hier verantwortlich machen. Ohne Ihr kontinuierliches Bestreben mich während meines Studiums in die Welt von Kautschuk und Gummi zu pushen, hätte mich mein Weg wohl nie hierher geführt. Danke dafür!

To the ETE-family. The team spirit and energy in our group always felt unique and special to me. I was very fortunate to experience this in the past $31 / 2$ years and moreover I am blessed to be part of you for a bit longer. Dear Jacques, Gin, Arqam, Kasia, Gina, Marcel, Chesidi, 
Xiaozhen, Hans, Indria, Ayush, Neven, Kumar, Kannika, Siti, Chai, Antonio, Nadia, Ivo, Jacob, Chigusa, Carmela, Zuzanna, Lukasz, Marcin, Priyanka, Alexandra, Karolina, Andrea, Sun and Stefan, thank you so much for sharing this important part of my life with me. On this occasion I do want to express my deepest feelings for the Dudes and my Polish Gang, it's purely a pleasure with you guys. Amir, short and simple explanations seem not to be your best friends. However, I do enjoy each discussion with you to a great extent. My dear Dorota, definitely the best party-buddy ever! Dear Marzieh, thank you for this terrifying experience of being your front passenger while driving on a test track. Never again! I really appreciate our daily funny teasing, it always puts a smile on my face. Lieber André, es ist schön auch im fremden Holland einen deutschsprachigen Freund zu haben. Dear Masaki, we almost spend and suffered our full PhD period together resulting in a friendship I highly appreciate and do not want to miss. My personal highlight in this time was our trip to New York, a unique experience I will not forget for the rest of my life. I really hope I will be able to visit you in Japan one day, as you visited me in my hometown. Dear Wisut, thank you for being such a great office mate and friend. Liebe Wilma, vielen Dank für all deine Unterstützung, Rat und Tat während dieser Phase meines Lebens. Ich hoffe in den kommenden Jahren viel von dem wiederzugeben und euch in diesem Universitätswahnsinn gebührend unterstützen zu können. To my beloved Ceciel: You are performing an outstanding job, on a professional as well as personal level. Amazing to have you around! Dries, Sexy. Thank you for being such an awesome technician and moreover a true friend. You made me realize that being happy and loving what you do is one of the most important things in life to achieve. Also thank you for the time-consuming support with the translation of my summary. Dear Louis, I especially appreciate your Dutch lessons teaching me the most "important" expressions to survive the daily life besides work! All of you, please take care of each other and yourself!

To my awesome paranymphs: Dear Rafal. Your "cutiful" creative mind and talent, contaminated by science, is inspiring on so many levels and it is a pleasure and honor to call you my friend! I'm so curious where our future will lead us and till then I promise to take advantage of our shared time to the best! Let's make life satisfactory and beyond! Cheers Bro! Dear little princess (Akansha). In the past couple of years you did not only become one, if not the best friend to me, but moreover you turned out to be my surrogate family and little sister. Without having you around, especially in the last couple of months, I hardly would have been able to finish this thesis. Thank you so much for being that special person and enrichment to me and my life!

Lieber Matze, auch wenn unsere Leben uns mittlerweile an unterschiedliche Orte geführt haben und die gemeinsame Zeit sehr rar geworden ist, genieße ich doch jede einzelne Minute, in der wir uns sehen. Jede Begegnung wirkt so vertraut wie früher. Ich hoffe, dass uns dies nie verloren geht. Danke für alles! Lieber Alex. Ich beneide dich um die stets positive Energie, Laune und Einstellung. Welche Hürden dir auch im Wege sind oder von dir selbst in den Weg gelegt wurden, du hast Sie gemeistert und dabei nie dein Lachen verloren. Verliere diese Fähigkeit bitte nie!

Family was and will always be my highest priority and of greatest importance in my life. And I feel infinitely blessed and fortunate for having them.

Liebe Oma, lieber Opa. Es ist ein Segen, dass ich mit Großeltern wie euch aufwachsen durfte. Danke für all die tollen Jahre! Liebe Moni, lieber Gerd, liebe Lena und lieber Lukas. Noch bevor ich überhaupt die Einladungen für meine Verteidigung schreiben konnte, hattet ihr euch schon gemeinsam angekündigt. Eure Unterstützung ist einfach überwältigend und ich bin unglaublich dankbar dafür! Liebe Erika und lieber Michael. Ich finde es überragend, dass ihr in den letzten Jahren mehr und mehr ein aktiver Teil meiner Familie geworden seid und kann es nicht erwarten, euch endlich in Wien besuchen zu kommen! Liebe Oma, Leinach war für mich insbesondere als Kind immer wie eine zweite Heimat und daran hattest du sehr großen Anteil. Ich danke dir für all diese schönen Erinnerungen, die ich nie vergessen werde! Lieber Joachim, es freut mich sehr zu sehen, dass du dein Glück und die Zufriedenheit in deinem Leben gefunden hast! 
Lieber Fritz, lieber Ben, liebe Paula, lieber Max, liebe Cinzia und Clara. Eure kleine Großfamilie in Erlangen gibt mir das Gefühl von Geborgenheit und Heimat. Bleibt so fantastisch, wie ihr seid!

Meine liebe Anna. Zu kaum einen anderen Menschen in meinem Leben fühle ich diese tiefe Vertraut- und Verbundenheit, die ich nur bedingt in Worte fassen kann. Und das obwohl wir uns viel zu selten sehen oder sprechen. Trotzdem verstehen wir uns blind und ohne Worte. Ich bin überglücklich, dass du ein Teil von mir bist! Liebe Antje, mehr als jeder andere Mensch in meinem Leben hast du mir vorgelebt und zu verstehen gegeben, was Familie bedeutet. Ich hoffe, ich bin in der Lage, diese Werte eines Tages an meine eigenen Kinder weiterzugeben.

Liebe Mama, lieber Papa. Dank euch hatte ich eine fantastische und unbeschwerte Kindheit, bin sicher und behütet aufgewachsen und trotz mancher Probleme während der Schulzeit konnte ich mich immer auf euch und eure 100\%ige Unterstützung verlassen. Ihr habt mir alle Freiheiten und Möglichkeiten eröffnet, diesen Werdegang einzuschlagen und durch euch bin ich geworden, wer ich heute bin. Und auch, wenn es nicht an mir ist, zu beurteilen, wie gut euch dieser Job gelungen ist, so kann ich doch eines sagen: Hier und jetzt stehe ich an einem Punkt in meinem Leben, der in jeglicher Hinsicht kaum besser sein könnte! Danke für die unendliche Hingabe, Unterstützung und Liebe, die Lorenz und ich durch euch an jedem einzelnen Tag unseres Lebens erfahren durften und dürfen! An mein Bruderherz: Insbesondere die vergangenen Jahre haben mir gezeigt, wie sehr ich dich und unsere gemeinsame Zeit vermisse. Ich bin unendlich stolz auf dich, auf all deine Leistungen, deinen Mut und deine Entschlossenheit und wünsche mir nichts mehr, als das uns unsere Zukunft wieder näher zusammenführt. Ihr alle erfüllt mich mit unbeschreiblicher Freude und bedeutet mir die Welt. Ich liebe euch!

Last but not least, I do want to address some words to both of my supervisors André and Anke. Euch beiden gebührt eigentlich je mindestens eine separate Seite in dieser Arbeit.

Lieber André. Mit deiner kritischen aber ebenso offenen und ehrlichen Art hast du mich auf so vielen Ebenen reifen und wachsen lassen. Die Trennung zwischen beruflichem und privatem Zusammenleben ist nicht immer leicht und dennoch ist auch dies dir fantastisch gelungen. Insbesondere habe ich unsere gemeinsamen Diskussionen genossen, bei denen ich mich trotz meiner fehlenden bzw. geringen Erfahrung immer gleichberechtigt und fair behandelt gefühlt habe und auch mit kritischen Aspekten nie zögerlich sein musste. Ich danke dir aus tiefstem Herzen für alles, was du für mich getan hast.

Liebe Anke. Ich hätte mir nie erträumen lassen, in meinem Leben einen Förderer und Mentor wie dich zu finden und schon gar nicht zu verdienen. Du siehst Dinge in mir und glaubst an meine Fähigkeiten, wie es sonst nur meine Mama im Stande ist zu tun. Darüber hinaus warst und bist du die letzten Jahre nicht nur ein großartiger Zuhörer, Therapeut und Lehrer, der mir unter anderem Diplomatie und Hochdeutsch näher gebracht hat, beides ist bis heute verbesserungswürdig, sondern warst mir vor allem eine wahre Freundin! Mein unendlicher Dank gilt dir für all das Vertrauen und deinen Glauben in mich, dies werde ich dir nie vergessen! Zu guter Letzt hast du dich auch noch durch meine Summary gequält und diese für mich ins holländische übersetzt. Hoffentlich bin ich eines Tages in der Lage, diese nicht nur zu lesen sondern auch zu verstehen :-).

With all these great people I am blessed to know and got to know in the past years there is unfortunately always a risk to miss someone even if he and/or she equally deserves my gratitude. Please forgive my imperfection. Representatively for everything all of you did for me I want to end this work with the most truthful and honest words I can think of. From the bottom of my heart:

Thank you! 
